\title{
PARALLEL OLAP ON MULTI/MANY-CORE AND CLOUD PLATFORMS
}

\author{
by \\ Hamidreza Zaboli \\ A thesis submitted to \\ the Faculty of Graduate Studies and Research \\ in partial fulfillment of \\ the requirements for the degree of \\ DOCTOR OF PHILOSOPHY \\ School of Computer Science \\ at

\section{CARLETON UNIVERSITY}

Ottawa, Ontario

March, 2014

(c) Copyright by Hamidreza Zaboli, 2013 


\section{Abstract}

One of the most powerful and prominent technologies for knowledge discovery in Decision Support System environments is Online Analytical Processing (OLAP). OLAP is the foundation for a wide range of essential business applications. Since its introduction, OLAP has consistently required a massive computational power. On the other hand, the physical limits of the speed for single processor systems bound the performance of any single processor solution. Parallel and distributed processing can provide two key ingredients to solve this problem: increased computational power through parallel processors and increased I/O bandwidth through parallel storage.

In this thesis, we provide new methods to parallelize OLAP systems on the recent parallel and distributed platforms. We provide new algorithms including two parallel sorting algorithms (an important part of data cube construction) on many-core Graphics Processors (GPUs) and multi-core CPUs. In addition, we introduce a method for parallel construction of static data cubes on multi-core CPUs.

Next, we present the main contribution of this thesis in the area of Real-time OLAP. We offer and discuss a new algorithmic solution with a new data structure called $P D C$-tree that supplies Real-time OLAP for multi-core platforms. To our knowledge, the PDC-tree is the first solution that provides a fully parallel Real-time OLAP using a parallelized tree data structure. We emphasize that the $P D C$-tree provides Real-time OLAP without materializing any data cube, and hence avoids its drawbacks.

In the last part of this thesis, we focus on the subject of parallel and distributed Realtime OLAP on cloud architectures. A cloud-based framework called CR-OLAP is developed that builds the structure of our cloud solution. CR-OLAP encompasses a new OLAP data structure called $P D C R$-tree, a non-trivial enhanced successor of the $P D C$-tree. In addition to answering OLAP queries in a real-time manner, $C R$-OLAP provides the scalability and load balancing of data among cloud resources, while assuring performance for very large data warehouses. Experiments on the Amazon EC2 Cloud confirm the real-time responsibility of CR-OLAP under a heavy load of OLAP queries in large data warehouses. 
To my parents 


\section{Acknowledgements}

First and above all, I am grateful to my supervisor, Dr. Frank Dehne who always had a welcoming attitude, and provided me with a great support in every step of my Ph.D. course. This thesis would not have been possible without his guidance and supervision.

Special thanks to Mikhail Genkin, Stephan Jou, and Robin Grosset from IBM Canada for their discussions and assistance in my IBM CAS project. Thanks for providing access to the test benchmarks and hardware facilities at IBM Canada.

I would also like to express my gratitude to Dr. Andrew Rau Chaplin for his productive discussions and feedback in the last tier of my project with IBM CAS Canada. Thanks to Rebecca Zou and Quan Kong for their discussions and programming expertise during the last part of this project.

My thoughts are with my wife, Arghavan, who has been withstanding the unpleasant

distance between us since I started to write this thesis. I am writing this in the hope of having her with me soon.

This work is supported by IBM Centre for Advanced Studies (CAS) Canada Research in the form of a CAS Research Fellowship with agreement number CAN2010-157. 


\section{Table of Contents}

$\begin{array}{ll}\text { Abstract } & \text { ii }\end{array}$

Acknowledgements $\quad$ iv

List of Tables $\quad$ ix

List of Figures $\quad$ x

$\begin{array}{ll}\text { Glossary } & \text { xviii }\end{array}$

$\begin{array}{lll}\text { Chapter } 1 \text { Introduction } & 1\end{array}$

1.1 Introduction . . . . . . . . . . . . . . . . 1

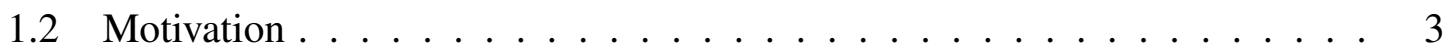

1.3 Statement of the Problems . . . . . . . . . . . . . . 4

1.3.1 Parallel Sorting on Multi/Many-core Platforms . . . . . . . . . . 4

1.3.2 Parallel Building of Static Data Cubes on Multi-core Platforms . . . 5

1.3.3 Real-time OLAP on Multi-core and Cloud Platforms . . . . . . . 6

1.4 Contributions ....................... 6

1.4.1 Parallel Sorting on Multi/Many-core Platforms . . . . . . . . . . 7

1.4.2 Parallel Building of Static Data Cubes on Multi-core Platforms . . . 7

1.4.3 Real-time OLAP on Multi-core and Cloud Platforms . . . . . . . 8

1.5 Overview of Results . . . . . . . . . . . . . . . . . . . 9

1.5.1 Parallel Sorting on Many/Multi-core Platforms . . . . . . . . . . 9

1.5.2 Parallel Building of Static Data Cubes on Multi-core Platforms . . . 10

1.5.3 Real-time OLAP on Multi-core and Cloud Platforms . . . . . . . . 11

1.6 Organization of Thesis . . . . . . . . . . . . . 13

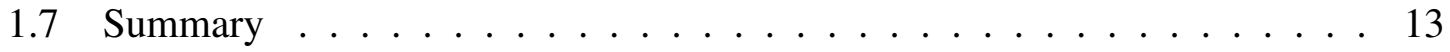


$\begin{array}{lll}\text { Chapter } 2 & \text { Background } & 14\end{array}$

2.1 Introduction . . . . . . . . . . . . . . . . . . . . 14

2.2 Parallel and Distributed Platforms _... . . . . . . . . . . . . 14

2.2.1 Multi and Many-core Platforms . . . . . . . . . . . . . 15

2.2.1.1 Multi-core CPUs . . . . . . . . . . . . 15

2.2.1.2 GPUs: Graphics Processing Units . . . . . . . . . . . 17

2.2.2 Cloud Platform . . . . . . . . . . . . . . . . 17

2.3 Data Warehousing and OLAP . . . . . . . . . . . . . . . 19

2.3.1 Decision Support Systems and OLAP . . . . . . . . . . . . 19

2.3.2 Data Cubes ....................... 20

2.3.3 Real-time OLAP . . . . . . . . . . . . . . 23

2.4 Multi-dimensional Data Indexing . . . . . . . . . . . . . . . . . 24

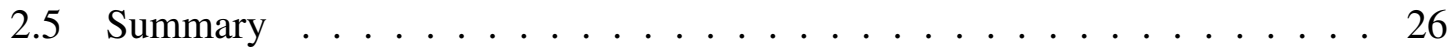

$\begin{array}{lll}\text { Chapter } 3 & \text { Literature Review } & 27\end{array}$

3.1 Introduction . . . . . . . . . . . . . . 27

3.2 Parallel Sorting on Multi/Many-core Platforms . . . . . . . . . . . . . 27

3.3 Parallel Building of Static Data Cubes on Multi-core Platforms . . . . . . . 28

3.3.1 Building Static Data Cubes . . . . . . . . . . . . . . . . . 29

3.3.2 Parallel Building of Static Data Cubes on Multi-core Platforms . . . 30

3.3.3 Parallel External Sorting on Multi-core Platforms . . . . . . . . . 31

3.4 Real-time OLAP . . . . . . . . . . . . . . . . 31

3.4.1 Real-time OLAP and Big Data Platforms . . . . . . . . . . . . 34

3.5 Summary . . . . . . . . . . . . . . . . 35

$\begin{array}{lll}\text { Chapter } 4 & \text { GPU BUCKET Sort } & 36\end{array}$

4.1 Introduction . . . . . . . . . . . . . . 36

4.1.1 Statement of the Problem . . . . . . . . . . . . . 36

4.2 GPU Bucket Sort: Deterministic Sample Sort For GPUs . . . . . . . . . . . 36

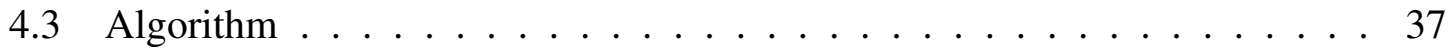

4.4 Experimental Results . . . . . . . . . . . . . . . . . . . . 40 
$4.4 .1 \quad$ Setup . . . . . . . . . . . . . . . . . 40

4.4 .2 Results . . . . . . . . . . . . . . . . . 41

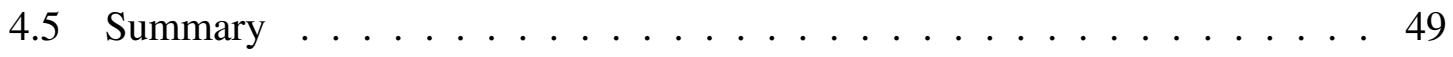

Chapter $5 \quad$ MCMD-Sort and MCMD-Cube $\quad 50$

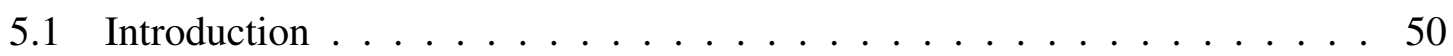

5.1.1 Statement of the Problem . . . . . . . . . . . . . . . 50

5.2 MCMD-CUBE Method . . . . . . . . . . . . . . . . . . . 51

5.3 MCMD-SORT Method . . . . . . . . . . . . . . . . . 52

5.3 .1 MCMD-Merge Method . . . . . . . . . . . . . . . . . 55

5.4 MCMD-CUBE Algorithm $\ldots \ldots \ldots \ldots \ldots$

5.5 Experimental Results . . . . . . . . . . . . . . . . 58

$5.5 .1 \quad$ Setup . . . . . . . . . . . . . . . . . 59

5.5 .2 Results . . . . . . . . . . . . . . . . . . . . . 59

5.5.2.1 Results for MCMD-SORT method . . . . . . . . . . 59

5.5.2.2 Results for MCMD-CUBE method . . . . . . . . . . 63

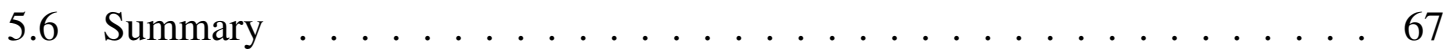

$\begin{array}{lll}\text { Chapter } 6 & \text { Real-Time OLAP } & 68\end{array}$

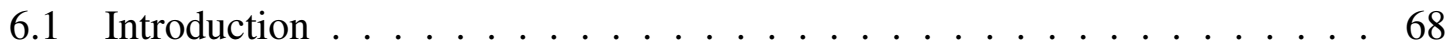

6.1 .1 Real-timeness . . . . . . . . . . . . . . . . . . . . 68

6.1 .2 Parallelness . . . . . . . . . . . . . . . . . 70

6.2 Real-Time OLAP on Multi-Core Platforms _ . . . . . . . . . . 71

6.2.1 Statement of the Problem . . . . . . . . . . . . . . . 71

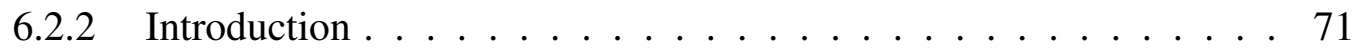

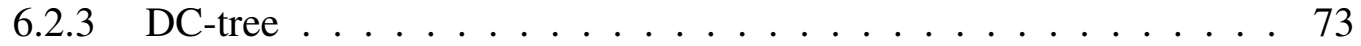

6.2 .4 PDC-tree . . . . . . . . . . . . . . . . . . . . 74

6.2 .5 Algorithms . . . . . . . . . . . . . . . . . . 77

6.2.5.1 Algorithm PARALLEL_OLAP_INSERT . . . . . . . . 77

6.2.5.2 Algorithm PARALLEL_OLAP_QUERY . . . . . . . 80

6.2.6 Experimental Results . . . . . . . . . . . . . . . . . . 83 
6.2 .6 .1 Setup . . . . . . . . . . . . . . . . 84

6.2.6.2 Results . . . . . . . . . . . . . . . . . . 85

6.3 Real-Time OLAP on Cloud Platforms _ . . . . . . . . . . . . . . 97

6.3.1 Statement of the Problem . . . . . . . . . . . . . . . . . . 97

6.3 .2 Introduction . . . . . . . . . . . . . . . . . . 97

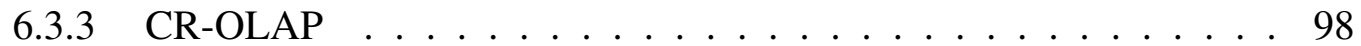

6.3.4 PDCR-tree . . . . . . . . . . . . . . . . . . . . . . 104

6.3 .5 Algorithms . . . . . . . . . . . . . . . . 110

6.3.5.1 Insertion Algorithms . . . . . . . . . . . . . . . . 110

6.3.5.2 Query Algorithms . . . . . . . . . . . . . . 110

6.3.6 Scalability and Load Balancing . . . . . . . . . . . . . 113

6.3 .7 STREAM-OLAP . . . . . . . . . . . . . . . . . . 116

6.3.8 Experimental Results . . . . . . . . . . . . . . . . . . . . 117

$6.3 .8 .1 \quad$ Setup . . . . . . . . . . . . . . 117

6.3.8.2 Experimental Evaluation On Amazon EC2 Cloud . . . . 122

6.4 Summary . . . . . . . . . . . . . . . . . . . . 132

$\begin{array}{lll}\text { Chapter } 7 & \text { Conclusion } & 133\end{array}$

7.1 Introduction . . . . . . . . . . . . . . . . . . 133

7.2 Summary of Results . . . . . . . . . . . . . . . . . . . . . 133

7.3 Observations . . . . . . . . . . . . . . . . . . . . 135

7.4 Future Work . . . . . . . . . . . . . . . . . . . . . . . . 137

7.4.1 Improvement on fault-tolerance $\ldots \ldots \ldots \ldots \ldots$

7.4.2 Scaling up to Big Data . . . . . . . . . . . . . 138

7.5 Publications . . . . . . . . . . . . . . . . . . . . . . . . . . . . 139

7.6 Achievements . . . . . . . . . . . . . . . . . . 140

Appendix A Correctness Proofs for Splitting Subtrees in PDCR-tree 141

$\begin{array}{ll}\text { Bibliography } & 148\end{array}$ 


\section{List of Tables}

Table 4.1 Performance Characteristics For Nvidia Tesla C1060, GTX 285 with 2 GB memory, GTX 285 with 1 GB memory, GTX 260 and Fermi GTX 480 (Source: [7]). . . . . . . . . . . . . . . . . . . 41

Table 5.110 pipes of the lattice of Figure reflattice 5-dimensional $\ldots . . . . .58$

Table 6.1 The two hardware platforms used in our experiments. . . . . . . . . 85

Table 6.2 Runtime comparison between PDC-Tree and multithreaded MySQL. Times shown are the total runtimes for the same sequences of 1,000 OLAP queries, with varied query coverages, on the same databases with 400,000 items. All data and queries are from the TPC-DS benchmark. ..................... . . 91

Table 6.3 Parallel OLAP insertion and query performance of the PDC-Tree as a function of the number of parallel threads for two query coverages, $5 \% \& 25 \%$, on a PDC-tree with 10 million data items for Intel Xeon Westmere EX. Number of queries and insertions are 10,000 and 1000, respectively. Same data as in Figure 6.12. Average transaction response times and associated speedup. . . . . . . . . . . . 96

Table 6.4 The optimal values of trees' parameters for the current Cloud setup. . 119 


\section{List of Figures}

Figure 2.1 Block diagram of a multi-core processor. Processing and storage units are labeled in the figure. In this diagram each processor has an L2 cache memory and two cores. Each core contains an L1 cache memory. ........................ 16

Figure 2.2 Block diagram of a Nvidia GPU. Source: www.nvidia.com. Processing and storage units are labeled in the figure. SP: Streaming Processor, L1: Shared cache between SP units, L2: Cache level 2, FB: Frame Buffer. . . . . . . . . . . . . . . . . 18

Figure 2.3 A comparison of the features of three distributed platforms: cluster, peer-to-peer, and cloud. . . . . . . . . . . . . . . . 19

Figure 2.4 Three-tiered OLAP model. . . . . . . . . . . . . . . . . . . 21

Figure 2.5 The lattice of a data cube with 4 dimensions A,B,C,D. This lattice includes 15 cuboids/sub-cubes/group-bys. . . . . . . . . . . 22

Figure 2.6 A three dimensional data cube for automobile sales data. . . . . . . 23

Figure 3.1 Hierarchy schema(left) and conceptual hierarchy(right) for dimension Customer. . . . . . . . . . . . . . . . 33

Figure 4.1 Illustration Of Step $7 \mathrm{In}$ Algorithm $1 \ldots$. . . . . . . . . . 40

Figure 4.2 Performance Of Deterministic Sample Sort For GPUs (GPU BUCKET SORT). Total runtime for varying number of data items on different GPUs: Nvidia Tesla C1060, GTX 260 and GTX 285. . . . . . . . . 42

Figure 4.3 Performance Of Deterministic Sample Sort For GPUs (GPU BUCKET SORT). Total runtime and runtime for individual steps of Algorithm 1 for varying number of data items. (a) For Nvidia GTX 285 and and (b) for Nvidia Fermi GTX 480 . . . . . . . . . . . . . . . 43

Figure 4.4 Runtime Of Algorithm 1 As A Function Of Selected Sample Size $s$ For Fixed $n=32 M, n=64 M$, and $n=128 M . \ldots \ldots 44$ 
Figure 4.5 Comparison between Deterministic Sample Sort (GPU Bucket Sort), Randomized Sample Sort (GPU S AMPLE SORT) [81] and Thrust Merge [98]. Total runtime for varying number of data items on an Nvidia GTX 285. ([98] and [81] provided data only for up to $16 \mathrm{M}$ and $32 \mathrm{M}$ data items, respectively.) . . . . . . . . . . . 46

Figure 4.6 Comparison between Deterministic Sample Sort (GPU Bucket Sort), Randomized Sample Sort (GPU S AMPLE SORT) [81] and THRUST MERge [98]. Total runtime for varying number of data items on an Nvidia Tesla C1060.([98] and [81] provided data only for up to $16 \mathrm{M}$ and $128 \mathrm{M}$ data items, respectively.) . . . . . . . . . 47

Figure 4.7 Performance of Deterministic Sample Sort (GPU Bucket Sort) for Different Input Data Distributions. . . . . . . . . . . . . . . . . 48

Figure 5.1 The lattice of a data cube with 4 dimensions A,B,C,D. This lattice includes 15 cuboids/sub-cubes/group-bys. . . . . . . . . . . 51

Figure 5.2 Input data division into smaller blocks of data. . . . . . . . . 53

Figure 5.3 Lattice of a 5-dimensional data cube. . . . . . . . . . . 57

Figure 5.4 Impact of growing data size on sorting time for different hardware configurations. Memory size: 8 GBytes, Record size: 8 bytes. (a) 1 disk, 1 processor core. (b) 2 disks, 2 processor cores. (c) 4 disks, 4 processor cores. . . . . . . . . . . . . . . . 60

Figure 5.5 Speedup curves for two data sizes, $32 \mathrm{~GB}$ and $128 \mathrm{~GB}$. Record size: 8 bytes, Memory size: 8 GB. . . . . . . . . . . . . . . . . 61

Figure 5.6 Impact of memory size on sorting time. Record size: 8 bytes, 4 disks, 4 cores. . . . . . . . . . . . . . . . . . . . 61

Figure 5.7 Impact of record size on sorting time. Total data size: $128 \mathrm{~GB}$. Memory size: 8 GB. 4 disks. 4 cores. . . . . . . . . . . . . . 63

Figure 5.8 Computing data cube of randomly generated data warehouse with 5 dimensions. Memory size: 8 GB. Record size: 164 bytes. . . . . . . 64 
Figure 5.9 Computing data cube on different data distributions. Memory size: 8 GB. Record size: 164 bytes. . . . . . . . . . . . . . . . . . 65

Figure 5.10 Computing data cube of TPC-DS benchmark. Memory size: 8 GB. Record size: 164 bytes. . . . . . . . . . . . . . . . . . . . 66

Figure 5.11 Speedup curve for computing data cube of TPC-DS benchmark. Fact table size: 124 GB. Memory size: 8 GB. Record size: 164

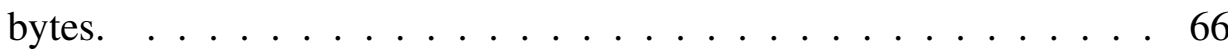

Figure 5.12 Impact of memory size on computing time of TPC-DS data cube. Fact table size: 124 GB. Record size: 164 bytes. . . . . . . . . . . . 67

Figure 6.1 A PDC-Tree: In order to process an input stream of OLAP insert and OLAP query transactions in real-time, we provide speedup through concurrent execution of transactions on a multi-core processor. . . . . . . . . . . . . . . . . 72

Figure 6.2 A 4-dimensional data warehouse with 3 hierarchy levels for each dimension. The first box for each dimension denotes the name of the dimension. . . . . . . . . . . . . . . . . . . . . . 75

Figure 6.3 Extended structure of a directory node for the PDC-tree. DC-tree entries (blue): $M D S$ (minimum describing set), $M$ (measure), link to each child node. Additional entries for the PDC-tree (red): TS (time stamp), Link to Sibling, MDS and TS for each child node link. 77

Figure 6.4 A directory node in PDC-tree and its fields in order from left to right: MDS: Minimum Describing Sequence, TS: Update Time Stamp, M: The set of measures, Links to children, Link to the sibling node rooted at the same parent. . . . . . . . . . . . . . . 77

Figure 6.5 Usage of stack and timestamp mechanism to detect updates on already counted nodes in an OLAP query. . . . . . . . . . . 83 
Figure 6.6 Parallel OLAP insertion performance of the PDC-Tree as a function of the number of parallel threads for Intel Sandy Bridge. (a) \& (b) Average transaction response time $\&$ throughput for 400,000 OLAP insertions to build an initial database. (c) \& (d) Average transaction response time $\&$ throughput for a subsequent 1,000 OLAP insertions into the built database. . . . . . . . . . . . . . . 86

Figure 6.7 Parallel OLAP transaction performance of the PDC-Tree as a function of the number of parallel threads for Intel Sandy Bridge. Mixed input of $I$ OLAP insertions and $Q$ OLAP queries. Queries aggregate $1 \%$ of database. (a) Average response time, linear scale. (b) Average response time, log-log scale. (c) Throughput, linear scale. (d) Throughput, log-log scale.

Figure 6.8 Parallel OLAP transaction performance of the PDC-Tree as a function of the number of parallel threads for Intel Sandy Bridge. Mixed input of $I$ OLAP insertions and $Q$ OLAP queries. Queries aggregate $5 \%$ of database. (a) Average response time, linear scale. (b) Average response time, log-log scale. (c) Throughput, linear scale. (d) Throughput, $\log -\log$ scale. . . . . . . . . . . . . . . . . 89

Figure 6.9 Parallel OLAP transaction performance of the PDC-Tree as a function of the number of parallel threads for Intel Sandy Bridge. Mixed input of $I$ OLAP insertions and $Q$ OLAP queries. Queries aggregate $25 \%$ of database. (a) Average response time, linear scale. (b) Average response time, log-log scale. (c) Throughput, linear scale. (d) Throughput, $\log -\log$ scale. . . . . . . . . . . . . . 90

Figure 6.10 PDC-tree insertions only performance as a function of the number of parallel threads for Intel Xeon Westmere EX. Stream of 14 million insertions into an initial PDC-tree with 1 million data items. (a) Throughput in log-log scale. (b) Average transaction response time in log-log scale. (c) Throughput in linear scale. (d) Average transaction response time in linear scale. . . . . . . . . . . . . 92 
Figure 6.11 Parallel OLAP insertion and query performance of the PDC-Tree as a function of the number of parallel threads for two query coverages, $5 \% \& 25 \%$, on a PDC-tree with 1 million data items for Intel Xeon Westmere EX. Number of queries and insertions are 1000 and 100, respectively. (a) Throughput in log-log scale. (b) Average transaction response time in log-log scale. (c) Throughput in linear scale. (d) Average transaction response time in linear scale. . . . . . . . 93

Figure 6.12 Parallel OLAP insertion and query performance of the PDC-Tree as a function of the number of parallel threads for two query coverages, $5 \%$ \& 25\%, on a PDC-tree with 10 million data items for Intel Xeon Westmere EX. Number of queries and insertions are 10,000 and 1000, respectively. (a) Throughput in log-log scale. (b) Average transaction response time in log-log scale. (c) Throughput in linear scale. (d) Average transaction response time in linear scale. . . . . . 95

Figure 6.13 Illustration of a distributed PDCR tree. . . . . . . . . . 100

Figure 6.14 The Hat in the master. By the next insertion in node $A_{1}$, the link between nodes $A_{1}$ and $A_{2}$ will be removed. This releases the two nodes and both nodes are then moved to workers to initiate two new subtrees(Fig. 6.15) . . . . . . . . . . . . . . . . . . . 101

Figure 6.15 Initiation of two new subtrees in two workers. (a) All nodes are still in the Hat. (b) Last insertion occurred in node $A_{1}$ and caused a horizontal and vertical directory node split. . . . . . . . . . . 102 
Figure 6.16 Growth of distributed PDCR-tree from an empty hat in the master and creation of subtrees in the workers. Solid black nodes represent data nodes and other nodes represent directory nodes. (a) The hat with 3 directory nodes in the master (no subtree/worker), (b) Insertion of a new data node and creation of a new directory node $(C)$. Node $B$ is now disconnected, however it is above the Cut Level, (c) Insertion of a new data node and creation of the directory node $D$. This case shows a transition stage. The depth of the hat has become greater than $h$. Subtrees $D$ and $A^{\prime}$ are now independent and may be sent to workers (Refer to Appendix A for proofs). (d) Two workers are allocated from the cloud environment by $C R-O L A P$, and subtrees are sent to the workers. (e) The subtrees in the workers grow by inserting new data records into distributed PDCR-tree. . . . . . 103

Figure 6.17 A 4-dimensional data warehouse with 3 hierarchy levels for each dimension. The first box for each dimension denotes the name of the dimension. . . . . . . . . . . . . . . . 105

Figure 6.18 The structure of an ID. The bits in each level are used to represent values for the entity and its ancestors in the upper hierarchy levels. The sample ID below represent a unique store located in the city of Los Angeles, in California, in the US. Note that concatenation of the bits in the levels builds up a large unique numerical value that represents the corresponding store. . . . . . . . . . . . . 108

Figure 6.19 The structure of IDs for the Store dimension with their coverages. Note that city values for each country starts from 1 , while Store S_keys are unique surrogate keys in the dimension. Lightly shaded area shows the coverage of the country 1 , while the dark shaded area is the coverage of the city 1 in country $1 \ldots$. . . . . . . . 108

Figure 6.20 A simple PDCR-tree with 2 dimensions Store and Date. Note both ordered and unordered dimensions can be treated as ranges, depending on the applied multi-dimensional indexing algorithms. . . . . . . 110 
Figure 6.21 A schematic picture of load balancing strategy. Load in worker w5 is detected above threshold. A subtree in the worker is selected for migration to worker $\mathrm{w} 3$ as destination worker. . . . . . . . . . . . . 117

Figure 6.22 8 dimensions of TPC-DS benchmark directly connected to the fact table "Store Sales". Boxes below each dimensions specifies hierarchy levels from 1 to 3 . Ordered dimensions are specified as "ordered" accordingly. . . . . . . . . . . . . . . . . . . . 120

Figure 6.23 Time for 1000 insertions as a function of the number of workers. $(N=40 \mathrm{Mil}, d=8,1 \leq m \leq 8)$

Figure 6.24 Time for 1000 queries as a function of the number of workers. $(N=$ $40 \mathrm{Mil}, d=8,1 \leq m \leq 8) \ldots \ldots \ldots \ldots \ldots$

Figure 6.25 Speedup for 1000 queries as a function of the number of workers. $(N=40 \mathrm{Mil}, d=8,1 \leq m \leq 8) \ldots \ldots \ldots \ldots \ldots$

Figure 6.26 Time for 1000 insertions as a function of system size: $N \& m$ combined. $(10 \mathrm{Mil} \leq N \leq 80 \mathrm{Mil}, d=8,1 \leq m \leq 8) \ldots \ldots \ldots$

Figure 6.27 Time for 1000 queries as a function of system size: $N \& m$ combined. (10Mil $\leq N \leq 80$ Mil $, d=8,1 \leq m \leq 8) \ldots \ldots$. . . . 125

Figure 6.28 Time for 1000 insertions as a function of the number of dimensions. $(N=40 \mathrm{Mil}, 4 \leq d \leq 8, m=8) \ldots \ldots \ldots 127$

Figure 6.29 Time for for 1000 queries as a function of the number of dimensions. The values for "1D-index 95\% coverage" are 828.6, 1166.4, 1238.5, 1419.7 and 1457.8, respectively. ( $N=40 \mathrm{Mil}, 4 \leq d \leq 8$, $m=8)$

Figure 6.30 Time for 1000 queries (PDCR tree) as a function of query coverages: $10 \%-90 \%$. Impact of value “*” for different dimensions. $(N=40 \mathrm{Mil}, m=8, d=8) \ldots \ldots \ldots \ldots \ldots \ldots$

Figure 6.31 Time for 1000 queries (PDCR tree) as a function of query coverages: $91 \%-99 \%$. Impact of value “*” for different dimensions. $(N=40 \mathrm{Mil}, m=8, d=8) \ldots \ldots \ldots \ldots$ 
Figure 6.32 Time comparison for 1000 queries (Ratio: 1D-index / PDCR tree) for query coverages $10 \%-90 \%$. Impact of value “*” for different dimensions. $(N=40 \mathrm{Mil}, m=8, d=8) \ldots \ldots . \ldots . . \ldots 129$

Figure 6.33 Time comparison for 1000 queries (Ratio: 1D-index / PDCR tree) for query coverages $91 \%-99 \%$. Impact of value “*” for different dimensions. $(N=40 \mathrm{Mil}, m=8, d=8) \ldots \ldots . \ldots 130$

Figure 6.34 Query time comparison for selected query patterns for dimension Date. Impact of value “*” for different hierarchy levels of dimension Date. $(N=40 \mathrm{Mil}, m=8, d=8)$. . . . . . . . . . . . 131

Figure 7.1 A multiple-master scheme for CR-OLAP. The first master at left receives the input stream from clients and dispatches a copy of only update operations (e.g. insertion) to the second master at right. . . . 138

Figure A.1 A subtree $s$ and branch $P$ inside it. . . . . . . . . . . . . . . . . . 142

Figure A.2 Branch $P$ before removing horizontal links $a$ and $c$. . . . . . . . . 144

Figure A.3 Branch $P$ after removing horizontal links $a$ and $c$. . . . . . . . 145

Figure A.4 Branch $P$ before horizontal split in node $B$. . . . . . . . . . . . 145

Figure A.5 Branch $P$ after horizontal split in node $B$. Node $B^{\prime}$ is created as the result of the split. . . . . . . . . . . . . . . . . 146

Figure A.6 Branch $P$ and its child branch rooted at node $D$. Note nodes in branch $D$ do not have any link to the neighbor bracnhes $A$ and $C$. . 147

Figure A.7 A vertical split at node $B^{\prime}$ created node $E$. Note branch $E$ is now independent from neighbors. . . . . . . . . . . . . . . 147 


\section{Glossary}

CR-OLAP Cloud-based Real-time OLAP

MCMD-CUBE Multi-core Multi-disk Cube

MCMD-SORT Multi-core Multi-disk Sort

PDC-tree Parallel DC-tree

PDCR-tree Parallel DC Range-tree

TPC-DS Transaction Processing Performance Council

DBMS Database Management System

DSS Decision Support System

ECU Elastic Computing Unit

GB Gigabyte

GPU Graphics Processing Unit

GS Global Sample

HDFS Hadoop Distributed File System

IT Information Technology

MBR Minimum Bounding Rectangle

MMBR Multiple Minimum Bounding Rectangles

OLAP Online Analytical Processing

OLTP Online Transaction Processing 
SIMD Single Instruction Multiple Data

SM Streaming Multiprocessor

STL Standard Template Library

STXXL Standard Template Library for Extra Large Data Sets

TS Time Stamp 


\section{Chapter 1}

\section{Introduction}

\subsection{Introduction}

Databases and database management systems have always played a key role in the growth and management of organizations by organizing enterprise data. Today, most organizations crucially rely on database systems for business operations. The recent changes to the science and economy over the past decade have further increased the significance of database systems. In fact, the analysis and querying of such data have become so demanding that have necessitated the introduction of a new subject on top of database systems called Business Analytics. Business analytics encompasses management, forecasting, marketing, budgeting, planning and many other issues related to the past and future performance of organizations. However, with the fast growth of databases in size, their management and analysis requires a high "computational power" and "storage capacity".

The recent competitive urge of database users for business analytics capabilities has further increased the necessity of the massive computational and storage capacities. In the past few years, large organizations have been dealing with data sizes in the scale of tera and petabytes. The reputable topic Big Data has been recently introduced as a response to the above requirements. Big Data is defined as the collection, storage, retrieval, and other related processes of such massive data sets. Business analytics in Big Data environments has become a "hot" topic in information technology (IT) and related contexts.

In order for database systems to handle such massive data, IT departments began to exploit rich new tools and paradigms for processing the wealth of data and information generated on their behalf. Along with relational databases, practitioners now look to advanced analytical tools in the hope of obtaining a competitive edge. This class of applications comprises what are known as Decision Support Systems ( DSS ). They are designed to empower users with the ability to make effective decisions regarding both the current and future state of an organization. In fact, DSS is a tool for business analytics. DSS must not 
only encapsulate static information, but it must also allow for the extraction of patterns and trends that would not be immediately obvious for users. The users must be able to visualize the relationships between the concepts such as customers, vendors, products, inventory, geography, and sales. Moreover, they must understand these relationships in a chronological context, since it is the time element that ultimately gives meaning to the concluded observations.

One of the most powerful and prominent technologies for knowledge discovery in DSS environments is Online Analytical Processing (OLAP). OLAP is the foundation for a wide range of essential business applications, including sales and marketing analysis, planning, budgeting, and performance measurement $[61,88]$. The processing logic associated with this form of analysis is encapsulated in what is known as the OLAP server. An OLAP server supports analyzing multidimensional data stored in a data warehouse from multiple views.

Given the above circumstances, it is clear that the emphasis in OLAP servers is on analytics, query processing, performance, and response time. However, if not impossible, it has become extremely difficult to satisfy such requirements in today's large data warehouses. On the other hand, the excessive demand of OLAP systems for high computational and storage capacities would simply break down the traditional processing models which are based on a single processor solution. Nevertheless, parallel and distributed processing platforms provide a higher computational and storage capacity for such massive computebound and I/O-bound tasks. Development of the recent parallel hardware platforms such as multi-core and cloud platforms has opened new sights for researchers and specialists.

In order to effectively exploit the parallel computational and storage capacities in OLAP systems, one needs to design and develop parallel software techniques and methods that utilize the parallel hardware. In this thesis, we aim at providing such methods. We offer new software solutions for building OLAP servers in large data warehouses using parallel and distributed processing. We present and discuss methods and techniques to handle the massive computational and storage needs of large OLAP servers. In the reminder of this chapter, we will briefly review related problems for building OLAP servers in multicore and cloud environments. Next, we will review our contributions to those problems and present our results. In the next chapters of this thesis, we will offer the new software 
methods and solutions for the stated problems.

\subsection{Motivation}

Nowadays, DSS constantly receive a large volume of data that needs proper storage, processing, and analysis in order to produce beneficial results and information. In the past decade, organizations observed a dramatic growth in data size demanding new methods of data processing in decision support systems. On the other hand, current solutions used in OLAP servers are barely capable of handling today's databases in large organizations. Response time in OLAP systems is still widely a serious issue in particular for Real-time OLAP systems. This shortage necessitated the development of new methods and technologies capable of satisfying the computational and storage requirements of decision support systems.

Parallel and distributed processing platforms provide a high computational and storage capacity for compute-bound and I/O-bound tasks. The recent hardware platforms such as multi-core and cloud have created hopeful sights for handling such tedious tasks. Multi-core platforms usually provide increased computational power through many single processing units that reside in a single machine and share a unified memory and storage. Cloud platforms on the other hand, provide both increased computational power and storage through stand-alone machines connected over a network.

The key idea is that parallel platforms in particular multi-core and cloud have a high potential to meet OLAP and/or any tedious data analysis task. More precisely, the increased computational power of the multi-core and cloud platforms (together with its increased storage capacity) can significantly support the computational and storage demands of OLAP systems. However, software techniques, data structures, frameworks, and methods that utilize such parallel platforms are still gaps to be filled. This introduces an interesting opportunity for researchers to study, explore, and develop new methods and approaches for the efficient utilization of the new parallel platforms. It creates a new way to look at challenges posed by today's OLAP systems, particularly Real-time OLAP.

In this thesis, we aim at providing new parallel software methods and solutions for supporting OLAP in multi-core and cloud platforms. We pay a particular attention to Realtime OLAP as recent scientific, business, and even political affairs have been exploiting 
Real-time OLAP in order to make quick decisions in response to the latest instant changes in the trend of input data.

\subsection{Statement of the Problems}

In this thesis, we consider the three following subjects:

1. Parallel sorting on parallel platforms

2. Parallel building of static data cubes

\section{Parallel real-time OLAP for parallel platforms}

Parallel sorting has been playing a major role in most computing problems in particular building data cubes for static OLAP. For the second subject, building data cubes has been a dominant method in the past decade for OLAP and data warehousing. The third problem, as the main subject in this thesis, is dedicated to Real-time OLAP. In the following sections, we present an overview of the three subjects mentioned above, and outline the issues associated with each subject, respectively.

\subsubsection{Parallel Sorting on Multi/Many-core Platforms}

Sorting has been one of the most fundamental problems in computer science. Throughout the evolution of computer science, there have been extensive studies on sorting methods and algorithms. Sorting is a key step in building static data cubes, which are central to most of the OLAP servers.

With the introduction of parallel platforms, parallel sorting methods have been devel-

oped to utilize increased computational power through parallel processing. Even though parallel sorting has been studied for a long time in the past, it has received an increased attention ever since the new multi/many-core processors were introduced. Recent Graphics Processing Units (GPU) are many-core processors composed of hundreds and thousands of single processors. They provide a parallel processing platform beside traditional CPUs. For a detailed review of the recent many-core graphics processor, see Chapter 2, Section 2.2.1.2. 
The first problem that we will address in this thesis is parallel sorting on many-core graphics processors (GPUs). As new GPUs are introduced to the computing market, new software methods and algorithms are developed to utilize these processors. The recent GPUs have shown to be competitor parallel processors for the multi-core CPUs. Providing a large parallel processing capability, GPUs proved to be qualified candidates versus the multi-core CPUs for the development of parallel algorithms.

The first issue discussed in this thesis will be the problem of parallel sorting on GPUs. We aim at a parallel sorting method that sorts data items using integer or real value keys on a many-core GPU. Since all of the data items in the GPU reside in its internal memory, we categorize our parallel sorting method as an internal sorting. Such a parallel sorting method may be applicable to many other problems in particular data warehousing and OLAP. This problem will be discussed in Chapter 4 where we will also offer our method.

\subsubsection{Parallel Building of Static Data Cubes on Multi-core Platforms}

Most of the OLAP servers rely upon a data model known as the data cube [56, 63]. Conceptually, the data cube allows users to view organizational data from different perspectives and at a variety of summarization levels. In practice, materialized data cubes can be massive. Building data cubes is often a very compute and data intensive operation. With ever increasing corporate databases in size, this poses a considerable challenge. Considering the physical limits on the speed of sequential processing systems, it is unlikely that single processor platforms can handle the massive size of the data cubes in the future OLAP systems.

On the other hand, multi-core processors have gained wide acceptance in the market and are now present in all computer systems. The high computational power of the multi-core processors meets the need of data cube building methods for a high computational power. This is the second problem that we will address in this thesis. We specialize this problem for building static data cubes and providing an algorithmic method using parallel sorting. We will provide a method for parallel building of data cubes on multi-core CPUs. Additionally,

we will address the problem of parallel external sorting using multi-core processors. The outcome of this is utilized in our data cube method. Chapter 5 discusses this problem and the methods offered. 


\subsubsection{Real-time OLAP on Multi-core and Cloud Platforms}

Most of the traditional OLAP research, and most of the commercial systems, follow the static data cube approach proposed by Gray et al. [57] and materialize all or a subset of the cuboids/group-bys of the data cube in order to ensure adequate query performance. Building the data cube can be a massive computational task, and significant research has been published on sequential and parallel data cube construction methods. However, the traditional static data cube approach has several disadvantages. For example, the OLAP system can only be updated periodically and in batches, e.g. once every week. Hence, the latest information cannot be included in the decision support process.

As a consequence, several practitioners have been hoping for an integrated OLAP/OLTP approach with a Real-time OLAP system that is updated instantaneously as new data arrives and always provides an up-to-date data warehouse for the decision support process (e.g. [28]). This is the third problem that we will address in this thesis. Such an approach may not materialize a data cube to build an OLAP server since the construction of data cubes often prevents OLAP servers from providing up-to-date and real-time responses. We recognize the third problem in this thesis as "How to build Real-time OLAP servers without materializing data cubes"? This problem will be addressed in Chapter 6 .

We divide this subject into two areas: the first one being Real-time OLAP on multi-core processors and the second, Real-time OLAP on cloud architectures. We aim at providing a fine-grain parallelism for the two parallel platforms at the level of algorithms and data structures. In order to effectively utilize cloud resources, any solution for a cloud environment must also take the scalability and load balancing issues into consideration. We will discuss these problems separately in Chapter 6 and will provide our solutions accordingly.

\subsection{Contributions}

In this section, we briefly review the methods and solutions proposed in the next chapters for OLAP and in particular Real-time OLAP. For each problem stated in Section 1.3, we provide a solution including methods, algorithms, and data structures. For detailed discussions of the offered solutions, please refer to Chapters 4,5 , and 6 , respectively. 


\subsubsection{Parallel Sorting on Multi/Many-core Platforms}

For the first problem in this thesis (i.e. parallel sorting on many-core GPUs), we present a new parallel sorting algorithm called GPU Bucket Sort which is based on a deterministic sorting method. GPU Bucket Sort uses the single processing units of GPUs called SM to divide unsorted data into the distinct chunks of sorted data, and merge them into a single list of sorted data. Unlike radix sort and traditional CPU bucket sort, GPU Bucket Sort is a comparison-sort. GPU Bucket Sort reads the unsorted chunks of the data from the global memory of the graphics card and sorts the chunks in the shared memory of the so called single multi-processor units. Final sorted data items are written back to the global memory at the end of the sorting.

We implemented GPU Bucket Sort in the CUDA programming environment to be executed on Nvidia GPUs. For technical details of Nvidia GPUs, we refer readers to Chapter 2, Section 2.2.1.2. Chapter 4 discusses the GPU Bucket Sort method along with the experimental evaluations for this method.

\subsubsection{Parallel Building of Static Data Cubes on Multi-core Platforms}

For the second problem in this thesis, we provide a parallel data cube construction method called MCMD-CUBE. MCMD-CUBE is based on the classical Pipe Sort [14] which decomposes the lattice into a set of chains called pipes, and computes the views in each chain through an external memory sort. The performance of our MCMD-CUBE method depends critically on parallel external memory sorting. At the core of our MCMD-CUBE method is

a new parallel sorting method termed $M C M D$-SORT for multi-core processors with parallel disks which outperforms previous sorting methods for multi-core processors. The $M C M D$ SORT applies a parallel merging process termed MCMD-Merge to merge sorted sublists in the shared memory of the multi-core processors.

For experiments, we exploited the MCMD-SORT in the MCMD-CUBE method and built two static data cubes using randomly generated data and TPC data set for decision support systems (i.e. TPC-DS) [109]. Detailed discussions on the MCMD-CUBE and MCMD-SORT methods are presented in Chapter 5. 


\subsubsection{Real-time OLAP on Multi-core and Cloud Platforms}

Real-time OLAP contributes to the main subject of this thesis. We study Real-time OLAP for two parallel platforms: multi-core processors and cloud architectures. We present two new data structures called PDC-tree and PDCR-tree, and a framework called CR-OLAP for building Real-time OLAP servers on multi-core processors and cloud platforms. The PDCtree and PDCR-tree methods integrate OLTP and OLAP sides of a data warehouse. We call these methods real-time in that they are able to answer OLAP queries instantaneously while other transactions (e.g. insertions) are instantaneously received in the OLAP server.

The PDC-tree and PDCR-tree methods answer OLAP queries using the most recent data inserted into the data warehouse system. It is guaranteed that no recently inserted data item in the data warehouse is ignored for query answering. This is an advantage of our realtime solution, since most current OLAP methods lack this feature, and answer queries using batch-loaded data that is not up-to-date. In addition, we guarantee it to answer all received queries in real-time (i.e. no query takes a long time to be answered). Since currently there is no standard threshold for the limits and boundaries of a real-time answer in Real-time $O L A P$, we consider the time restriction of 1.0 second as real-time. For details regarding the definition of real-time responses, please refer to Chapter 6.

We present the PDC-tree as a Real-time OLAP method for multi-core processors. The $P D C$-tree is a tree data structure that stores and indexes inserted data in a data warehouse. The PDC-tree includes parallel algorithms for parallel OLAP operations. The PDC-tree and its algorithms provide a fine-grain parallelism for concurrent transactions that occur in the data warehouse. As the tree stores all inserted data records, it allows threads to concurrently traverse the tree and answer OLAP queries.

While the PDC-tree is developed for multi-core platforms, the PDCR-tree and $C R$ $O L A P$ provide Real-time OLAP for cloud platforms. The $C R-O L A P$ plays a structural role for our cloud-based Real-time OLAP server. In addition, we present the PDCR-tree as a non-trivial successor of the PDC-tree. The PDCR-tree is applied inside the CR-OLAP and operate as the core component in our Real-time OLAP server. The CR-OLAP is able to efficiently answer OLAP queries in real-time in large data warehouses. In addition, it is able to provide OLAP functionalities in real-time and use cloud resources to balance storage and computational requirements of data warehouse systems. The CR-OLAP provides 
scalability in cloud environments as well as load balancing mechanisms to guarantee a high performance and reliability. Detailed discussions on the PDC-tree, PDCR-tree, and CR-OLAP are presented in Chapter 6.

\subsection{Overview of Results}

In this section we present a summary of the results for each subject of this thesis outlined in Section 1.3. For each subject, we applied a proposed method discussed in Section 1.4 to a standard test benchmark and collected performance data. A summary of the collected results and our conclusions for each subject are presented in the following sub-sections. Our methods and achieved results are already published in peer-reviewed conferences and journal publications. We note our publications for each subject at the end of each subsection.

\subsubsection{Parallel Sorting on Many/Multi-core Platforms}

We developed GPU Bucket Sort, a new parallel sorting algorithm for many-core GPUs. Implementations are performed in the CUDA programming environment for Nvidia GPUs. Tests and experiments are performed on synthetic data with different sizes and different types of distributions. We repeated the same set of tests for other GPU sorting methods and compared the performance of the tested methods. Our experiments demonstrated that the GPU Bucket Sort outperforms the state-of-the-art GPU sorting methods at the time of development. The experiments are performed for a variety of test cases such as different types of data distributions including uniform, Gaussian, and Zipfian. Other experiments include the tests on different Nvidia GPUs, parameter optimization tests, and the tests for increasing data size. In all cases, the GPU Bucket Sort is compared with the GPU Sample Sort [81] and the Thrust Merge Sort [98]. The two methods were state-of-the-art GPU sorting methods at the time of development. Our results indicated that in most cases, the GPU Bucket Sort outperforms other GPU sorting methods.

We observed that in a few cases the GPU Bucket Sort gains nearly the same performance as the GPU Sample Sort. However the GPU Bucket Sort has the advantage of being deterministic over the GPU Sample Sort which is a randomized sort. The performance of the randomized GPU Sample Sort fluctuates with the input data distribution whereas the 
GPU Bucket Sort does not show such fluctuations. The GPU Bucket Sort showed a fixed sorting rate (number of sorted data items per time unit) for the entire range of data sizes tested (up to $n=512 M$ data items), whereas it is shown in [81] that the sorting rate for the randomized GPU Sample Sort fluctuates and often starts to decrease for larger values of $n$. We will discuss the GPU Bucket Sort method and its experimental evaluations in detail in Chapter 4.

GPU Bucket Sort method and achieved results are published as a journal paper titled "Deterministic sample sort for GPUs". Publication details can be found in [50].

\subsubsection{Parallel Building of Static Data Cubes on Multi-core Platforms}

We developed $M C M D$-CUBE method, a new parallel data cube construction method for multi-core processors with multiple disks. The $M C M D$-CUBE method exploits a parallel external sorting algorithm called MCMD-SORT developed by the author. The MCMDSORT outperforms other parallel external sorting algorithms for multi-core platforms. We used this sorting algorithm inside the $M C M D$-CUBE method to build static data cubes.

Our experiments were performed for an Intel Sandy Bridge multi-core processor with four parallel disks. We also compared the MCMD-SORT method with a state-of-the-art external sorting method for multi-core processors from STXXL [9] library. A comparison of results shows that the MCMD-SORT outperforms the sort method in the STXXL library. Note that the STXXL was one of the fastest parallel external sorting methods for multi-core processors at the time of development. We used the MCMD-SORT in the MCMD-CUBE method and built static data cubes using synthetic data and also TPC-DS [109] benchmark data set. Experimental results for a Sandy Bridge multi-core processor with four parallel disks indicated that the $M C M D-C U B E$ achieves $50 \%$ of the theoretically optimal linear speedup by increasing the number of processors and parallel disks. We discuss the $M C M D$ CUBE method in detail in Chapter 5.

MCMD-CUBE method is published as a conference and a journal paper titled "Parallel data cubes on multi-core processors with multiple disks" in [49], and "Parallel construction of data cubes on multi-core multi-disk platforms" in [51]. 


\subsubsection{Real-time OLAP on Multi-core and Cloud Platforms}

Real-time OLAP on Multi-core Processors: We developed the $P D C$-tree data structure and new algorithms for Real-time OLAP on multi-core platforms. The PDC-tree allows for multiple insert and multiple query operations to be executed concurrently and in realtime. It is able to answer large OLAP queries that involve roll-up, drill-down, slicing, and dicing in traditional data warehousing methods (e.g. data cubes). To our knowledge, this is the first Real-time OLAP system of this type that has been parallelized and optimized for multi-core processors, providing an opportunity for Real-time OLAP in large scale data warehouses.

We evaluated the $P D C$-tree method for many scenarios and collected the experimental results under a variety of test conditions. Input data for the Real-time OLAP server was provided by the TPC-DS benchmark data set [109]. We performed the tests on two multicore platforms: Intel Sandy Bridge processor and Intel Xeon Westmere EX processor with 4 and 20 processor cores, respectively. According to the test results, the $P D C$-tree achieved a significant speedup in transaction response time and a significant increase in transaction throughput for OLAP operations including both insertions and queries. The results confirmed that the $P D C$-tree answers every OLAP query in real-time in less than 1 second. In a test case for a database with 10 million data records, the $P D C$-tree achieved a speedup of nearly 20 on the Intel Xeon Westmere EX processor with 20 processor cores.

Other test cases included different query coverages in which the PDC-tree received OLAP queries with 3 different query coverages: $1 \%, 5 \%$, and $25 \%$. Throughout the tests, the longest response time was recorded at around 0.25 seconds which is considered realtime (a definition for real-time responses will be provided in Chapter 6). We also compared the $P D C$-tree method with $M y S Q L D B M S$ [5] to answer OLAP queries with different number of threads and different query coverages. The results showed that the $P D C$-tree answers OLAP queries faster than the $M y S Q L$ in most cases for 4 query coverages out of 5 cases.

Our new parallel real-time OLAP method has the potential to support Real-time OLAP servers in large data warehouses. We will discuss the details of the proposed algorithms, the $P D C$-tree data structure and our experimental evaluations in Chapter 6, Section 6.2. $P D C$-tree method is published as a conference paper titled "Parallel real-time OLAP on 
multi-core processors" in [41]. A journal version of the paper is to appear in the International Journal of Data Warehousing and Mining (IJDWM) titled as "Parallel Real-Time OLAP on Multi-Core Processors".

Real-time OLAP on Cloud Architectures: As the second project within this subject, we developed a framework called CR-OLAP and a data structure called PDCR-tree for Real-time OLAP in cloud environments. We built the CR-OLAP as a scalable framework for cloud architectures. The PDCR-tree, as a successor of the PDC-tree plays the role of an OLAP engine at the heart of each cloud node. The CR-OLAP scales up by increasing the size of the network, and allocates new cloud nodes to ensure a high performance in particular a real-time response for every OLAP query.

Experiments were performed on the Amazon EC2 Cloud [1] using the well-known TPC$D S$ benchmark. We increased the total number of the data records in the system up to 80 million records distributed in 8 cloud nodes. The CR-OLAP underwent a complete set of test cases including the impact of the number of cloud nodes, the impact of stored data size, the impact of system size, the impact of the dimensionality of data warehouse, together with a variety of test cases for OLAP queries. All of the test cases were accomplished with different query coverages. The $C R-O L A P$ was found to be particularly efficient for complex OLAP queries that need to aggregate a large portion of the data warehouse, such as "Report the total sales in all stores located in California and New York during the months February-May of all years". In the worst case, the CR-OLAP answered the largest OLAP query with 85 different values in 8 dimensions covering $95 \%$ of each dimension, in a data warehouse with 80 million records distributed over 8 machines in 0.3 seconds.

The tests also indicated that the $C R-O L A P$ scales well with increasing the number of workers. For example, for a fixed data warehouse size (10 million data items), when the number of workers is increased from 1 to 8 , the average query throughput and latency improves by a factor 7.5 which is considered nearly a linear speedup. The CR-OLAP also responds well to the high dimensionality of the data warehouse. For tree data structures this is a critical issue as it is known e.g. for R-trees that, with increasing the number of dimensions, even simple range search (no dimension hierarchies, no aggregation) can degenerate to a linear search (e.g. [47]). In our experiments, we observed that increasing 
the number of dimensions does not significantly impact the performance of the CR-OLAP. We will discuss details of the CR-OLAP and PDCR-tree in Chapter 6, Section 6.3.

PDCR-tree method is published as a conference paper titled "A Distributed Tree Data Structure For Real-Time OLAP On Cloud Architectures” in [48].

\subsection{Organization of Thesis}

In Chapter 2, we present the necessary background and fundamentals of the software methods and hardware platforms used in this thesis. Chapter 3 reviews the methods and previous studies relevant to the three subjects of this thesis. We do not review all traditional methods in this chapter as some of the addressed problems root back to fundamental problems in computer science. We rather focus on the recent studies for each subject.

Next, we discuss the first subject of this thesis: Parallel Sorting on Many/Multi-core Platforms in detail in Chapter 4. We present the GPU Bucket Sort method and our experimental results for this method in this chapter. Chapter 5 presents the $M C M D-C U B E$ and MCMD-SORT methods for Parallel Building of Static Data Cubes on Multi-core Platforms. Chapter 6 addresses the main subject of this thesis: Real-time OLAP on Multi-core and Cloud Platforms. In this chapter, we present a definition for Real-time OLAP, and then we discuss the PDC-tree, PDCR-tree, and CR-OLAP framework for multi-core and cloud platforms in two consequent sections. Following each method, we present our experimental results. At last in Chapter 7, we outline conclusion remarks and potential future studies.

\subsection{Summary}

In this chapter, we provided readers with an overview of OLAP, Real-time OLAP and data warehousing paradigms. We discussed three OLAP-related subjects along with the reasons for why we are interested in those subjects. A review of contributions and achieved results were also presented in this chapter. 


\section{Chapter 2}

\section{Background}

\subsection{Introduction}

In this chapter, we provide a survey of the fundamentals and background for the problems discussed in Chapter 1. We provide our survey in two folds. First, we discuss the recent advancement of hardware technologies from the perspective of processing and storage units. We review recent parallel and distributed platforms including multi/many-core processors and cloud platforms. It is necessary to provide readers with a brief review of such details since software methods proposed in this thesis are built for these platforms.

We provide an overview of the architectures of multi-core CPUs and many-core GPUs in particular Nvidia GPUs. We also present a brief discussion on cloud architectures. In the next section, we provide the software background of the problems and methods that we addressed in this thesis. Data warehousing, OLAP and building data cubes, real-time OLAP and finally multi-dimensional data indexing are the subjects that we review in the following sections.

\subsection{Parallel and Distributed Platforms}

The excessive demand of current OLAP systems for computational and storage power would simply break down traditional single processor solutions. Parallel and distributed processing platforms provide a higher computational and storage capacity for such massive compute-bound and I/O-bound tasks. The recent development of such hardware platforms (e.g. multi-core, grid, cloud) has attracted researchers to the area. However, in order to effectively exploit the elevated computational and storage capacities of parallel platforms, one needs to design and develop "parallel software techniques and methods" to enable OLAP servers in today's data warehouses. In the two following sections, we present a review of the two most recent parallel hardware technologies multi/many-core processors 
and cloud platforms.

\subsubsection{Multi and Many-core Platforms}

Multi-core platforms have gained a considerable attention since the early years of the past decade. Efforts for increasing the clock speed of processors seem to have been unsuccessful in the past decade [72]. Rather, computer architects seek a new way to increase the performance of processors. Integrating additional processor cores into a single processing unit provides additional computation power. This can also save the ruinous power consumption caused by increasing the clock speed of single-core processors. According to a study in [72], every $400 \mathrm{MHz}$ increase in the clock speed requires a $60 \%$ increase in the power consumption. Embedding multiple processor cores in a single chip provides parallel processing capabilities. In the past decade, manufacturers such as Intel and AMD introduced commercial processors containing multiple independent processors cores. Each

processor core is a stand-alone processing unit able to run ordinary CPU instructions. Processor cores can run multiple instructions at the same time enabling parallel processing for softwares and applications. However, the parallel performance of such processing units is bounded by Amdahls law [16].

\subsubsection{Multi-core CPUs}

Multi-core processors are comprised of independent processing units called processor cores or simply cores. The cores inside a multi-core processor are usually connected using a fast bus which may be separated from the system bus. The cores may have private or shared cache memories, or a combination of the two. The shared bus between the cores is connected to the main memory of the system and provides a slower connection with the memory. Multi-core processors usually share the main memory between their cores and are therefore called shared memory multi-core processors. Multi-core processors with the described architecture, introduced a new parallel platform for parallel softwares and applications. Figure 2.1 illustrates the block diagram of a multi-core processor. Intel multi-core processors provide a feature called Hyper-threading technology in which a processor core has two or more register banks [85]. Each hardware thread has one register bank and can be switched with another thread when it requests a memory access. This technology saves 


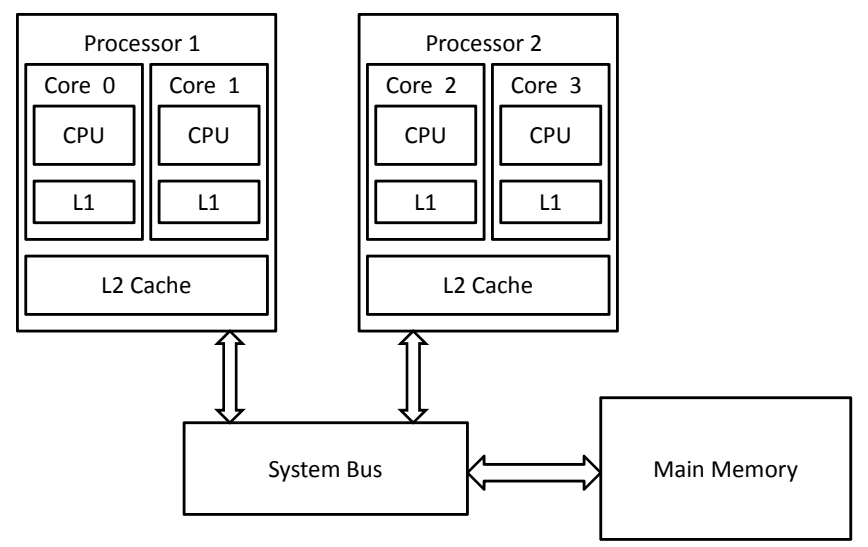

Figure 2.1: Block diagram of a multi-core processor. Processing and storage units are labeled in the figure. In this diagram each processor has an L2 cache memory and two cores. Each core contains an L1 cache memory.

the CPU time since other threads can replace the waiting threads. It has been reported that using Hyper-threading for applications provides an increased performance of $15 \%$ to $30 \%$ compared to the applications that do not use Hyper-threading [85]. In addition to Intel multi-core processors, other manufacturers also produced multi-core processors, including POWER4-POWER7 processors of IBM, ULTRASPARC processors by Sun Microsystems, and AMD multi-core processors. The number of cores inside multi-core processors is increasing every year leading to a higher computation power. High-end multi-core processors of Intel currently have 8 and 16 processor cores. Nvidia GPUs may also be classified as multi-core processors, although they are more often referred to as many-core processors due to the high number of single processing units (more than 1000) inside a GPU. For a brief review of many-core GPUs, refer to Section 2.2.1.2 of this Chapter. 


\subsubsection{GPUs: Graphics Processing Units}

In this section, we review Nvidia's unified graphics and computing platform for GPUs [83] and associated CUDA programming model [6]. However, the discussion applies more generally to GPUs that support the OpenCL standard [12]. A GPU consists of an array of streaming processors called Streaming Multiprocessors (SMs). Each SM contains several processor cores and a small size, low latency, local shared memory that is shared by its processor cores. All SMs are connected to a global DRAM memory through an interconnection network. The global memory is arranged in independent memory partitions and the interconnection network routes the read/write memory requests from the processor cores to the respective global memory partitions, and the results back to the cores. Each global memory partition has its own queue for memory requests, and arbitrates among the incoming $\mathrm{read} / \mathrm{write}$ requests, seeking to maximize DRAM transfers. This is performed by grouping read/write accesses to neighboring memory locations referred to as coalesced global memory access. Memory latency to global DRAM memory is optimized when parallel $\mathrm{read} /$ write operations can be grouped into a minimum number of sub-arrays of contiguous memory locations. It is important to note that data accesses from processor cores to their SM's local shared memory are at least an order of magnitude faster than accesses to global memory.

Figure 2.2 shows the block diagram of an Nvidia Fermi GPU which is the latest generation of Nvidia many-core GPUs. The above processing and storage units are indicated in the figure. The recent generation of Nvidia GPUs has up to 3072 processor cores and $4 \mathrm{~GB}$ of global memory[87].

\subsubsection{Cloud Platform}

Cloud computing has recently been the centre of attention in the field of parallel and distributed processing, and is rapidly evolving into a common source of computation and storage for organizations. Cloud computing is the presentation of computation and storage as a service to clients. It is a new parallel platform with high scalability and computation power compared to other models of parallel computing. In a cloud system, a group of computational and storage nodes are coupled together to provide specific services for clients. 


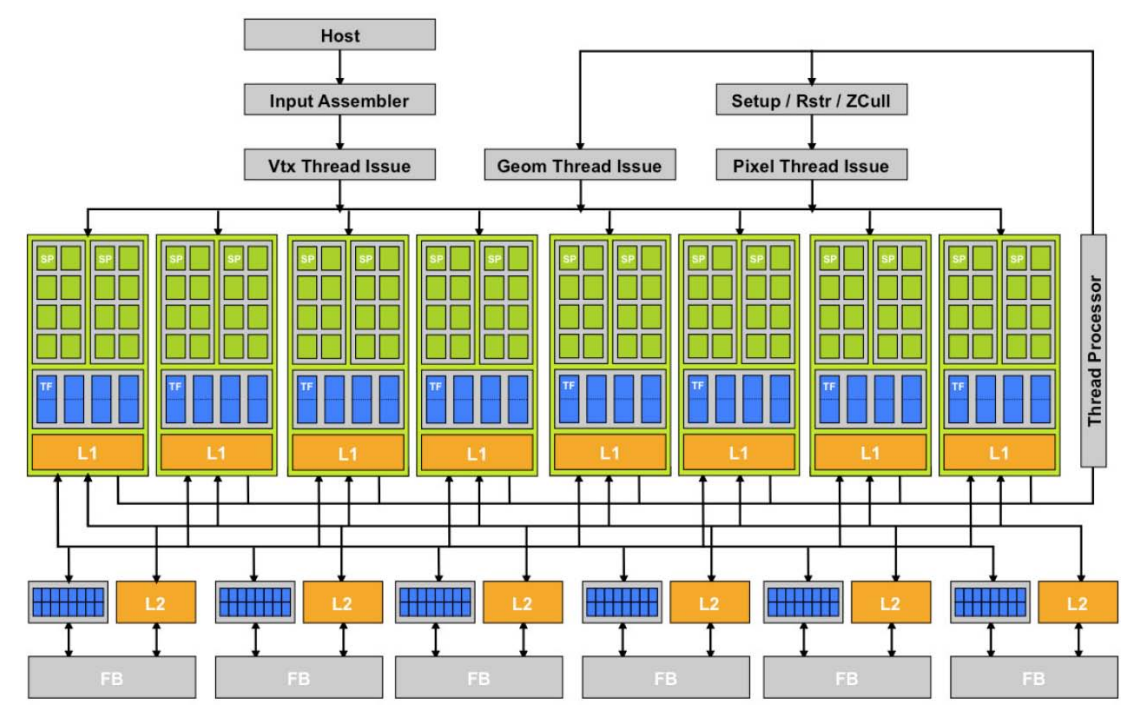

Figure 2.2: Block diagram of a Nvidia GPU. Source: www.nvidia.com. Processing and storage units are labeled in the figure. SP: Streaming Processor, L1: Shared cache between SP units, L2: Cache level 2, FB: Frame Buffer.

Clients are usually separated from cloud nodes; however depending on the type of application, some of the nodes may play client and server roles for each other. When clients request a service from the cloud system, it manages to provide the service by involving one or more cloud nodes in the process using software methods and algorithms. Cloud nodes may leave or join the cloud; however the system is responsible for providing fault-tolerance and redundancy techniques for cloud nodes. Providing replicas of data in cloud nodes is usually used as a redundancy strategy. Such replicas may be available in one or more than one node of the system. Compared to other parallel networked systems, such as cluster, peer-to-peer, and grid, we observe the differences summarized in Figure 2.3 between these platforms.

One difference between cloud and peer-to-peer systems is that nodes in a peer-to-peer system join the network for their own purposes (for example to receive a service), while in cloud, nodes join the system to work as part of the system and provide a service. Another important difference between cloud and the two other systems is in elasticity. Elasticity refers to the ability to assign more/less resources during a process in order to assure performance and computational/storage power for a requested service. This feature is not available in clusters and peer-to-peer networks. Grids share more features with cloud systems 


\begin{tabular}{|c|c|c|c|}
\hline Deature & Clatform & $\begin{array}{c}\text { Peer-to- } \\
\text { peer }\end{array}$ & Cloud \\
\hline Distributed & $X$ & $X$ & $X$ \\
\hline Scalable & & $X$ & $\times$ \\
\hline Elastic & & & $\times$ \\
\hline
\end{tabular}

Figure 2.3: A comparison of the features of three distributed platforms: cluster, peer-topeer, and cloud.

than peer-to-peer systems. A Grid system provides distributed computational power and storage as cloud; however it is usually more heterogeneous than cloud and is not presented as a service to clients. In the past few years, many companies offered processing and storage services using cloud systems. Google File System [32, 2], and Amazon EC2 Cloud [1] are examples of major cloud providers. Considering the advantages of cloud computing, many researchers and practitioners have shown their interest in extending and addressing scientific problems in this new environment. This is encouraging, particularly since cloud computing provides a high parallel computational power compared to previous hardware platforms. The elevated computational power of cloud systems and the distributed storage capacity provide organizations with a new powerful platform leading to an up-shift in IT.

\subsection{Data Warehousing and OLAP}

In this section we provide the basics of a large class of subjects and problems in IT, computer science and engineering known as data warehousing and OLAP along with related topics.

\subsubsection{Decision Support Systems and OLAP}

In the recent years, large organizations have been dealing with data sizes in the scale of tera and petabytes. The reputable topic Big Data is a direct response to the above requirements. Big Data is defined as the collection, storage, retrieval, and other related processes 
of such massive data sets. Business Analytics for Big Data has become a hot topic in information technology and related contexts. In order to handle requirements of such massive data, IT departments have began to exploit rich new tools and paradigms for processing the wealth of data and information generated on their behalf. Along with relational databases, the venerable cornerstone of corporate data management, knowledge workers and business strategists now look to advanced analytical tools in the hope of obtaining a competitive edge. This class of applications comprises what are known as Decision Support Systems (DSS). They are designed to empower the user with the ability to make effective decisions regarding both the current and future state of an organization. To do so, the DSS must not only encapsulate static information, but it must also allow for the extraction of patterns and trends that would not be immediately obvious. Users must be able to visualize the relationships between such things as customers, vendors, products, inventory, geography, and sales. Moreover, they must understand these relationships in a chronological context, since it is the time element that ultimately gives meaning to the observations that are formed.

One of the most powerful and prominent technologies for knowledge discovery in DSS environments is Online Analytical Processing (OLAP). OLAP is the foundation for a wide range of essential business applications, including sales and marketing analysis, planning, budgeting, and performance measurement $[61,88]$. The processing logic associated with this form of analysis is encapsulated in what is known as the OLAP server. By exploiting multi-dimensional views of the underlying data warehouse, the OLAP server allows users to "drill down" and "roll up" on hierarchies, "slice and dice" particular attributes. It also enables various statistical operations such as ranking and forecasting. Figure 2.4 illustrates the basic model where the OLAP server represents the interface between the data warehouse proper and the reporting and display applications available to end users.

\subsubsection{Data Cubes}

To support the mentioned functionality, OLAP relies heavily upon a data model known as the data cube [56,63]. Conceptually, the data cube allows users to view organizational data from different perspectives and at a variety of summarization levels. It consists of the base cuboid, the finest granularity view containing the full complement of $d$ dimensions (or attributes), surrounded by a collection of $2^{d}-1$ sub-cubes/cuboids that represent the 


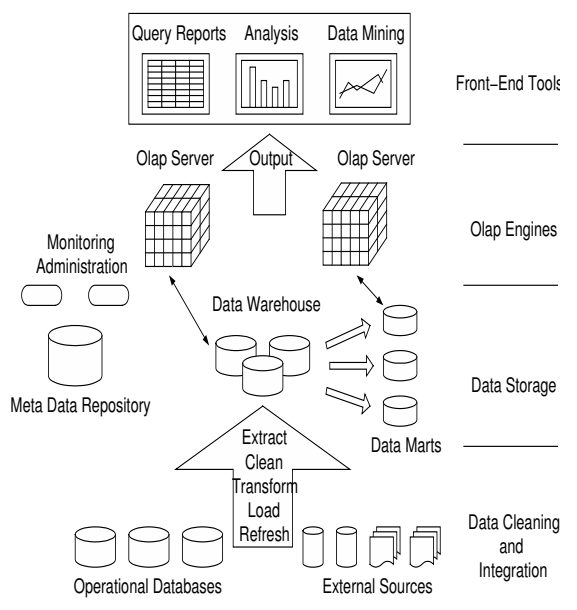

Figure 2.4: Three-tiered OLAP model.

aggregation of the base cuboid along one or more dimensions. Figure 2.6 illustrates a small four-dimensional data cube that might be associated with the automotive industry. In addition to the base cuboid, one can see a number of various planes and points that represent aggregation at coarser granularity. Note that each cell in the cube structure corresponds to one or more measure attributes (e.g. total sales).

The OLAP operations referred in the previous section (Section 2.3.1) are critical functionalities of a data cube. In a data warehouse containing $d$ dimension tables and a fact table $F$, we define these OLAP operations as follows. "Drill down" operation is defined as changing the view over the database from a summary level to a more detailed level in one or more dimensions of the data warehouse. "Roll up" operation is defined as the reverse operation to the "drill down". It changes the view up to a more summarized level. "Slice" operation is defined as picking a single value for one of the attributes that connects the fact table to a dimension table. By choosing a single value, the view over the database is changed from $d$ dimensions to $d-1$ dimensions. A "slice" operation does not change the summarization level for any dimension. "Dice" operation is defined as picking multiple values of multiple dimension tables in order to create a sub-cube of the data cube. The cuboids of a data cube are in fact the result sets of the mentioned OLAP operations. These pre-calculated cuboids provide an OLAP system with pre-calculated answers to most OLAP queries, and hence speed up query processing in the OLAP system. 


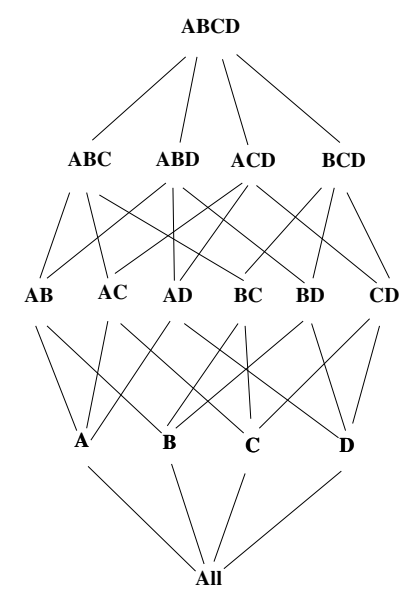

Figure 2.5: The lattice of a data cube with 4 dimensions A,B,C,D. This lattice includes 15 cuboids/sub-cubes/group-bys.

In addition, we define the fact table in an OLAP system as a table containing the measures and facts that are important in an organization. A fact table contains the key measures in enterprise data. For example, in a business process, sales and sale values are key measures that play a critical role in decision making. Fact table in a data warehouse system is the center of the database schema. It is directly or indirectly connected to all dimension tables of the data warehouse through an attribute, usually a foreign key. Fact table often contains the most detailed level data in the data warehouse system.

Typically, the collection of cuboids is represented as a lattice [63] of height $d+1$. Starting with the base cuboid - containing the full complement of dimensions -, the lattice branches out by connecting every parent node with the set of child nodes/views that can be derived from its dimension list. Figure 5.1 shows the lattice of a 4-dimensional data cube.

In general, a parent containing $k$ dimensions can be connected to $k$ views at the next level in the lattice. In principle, no special operators or SQL extensions are required to take a raw data set, composed of detailed transaction-level records, and turn it into a data structure, or group of structures, capable of supporting subject oriented analysis. Rather, the SQL group-by and union operators can be used in conjunction with $2 d$ sorts of the raw data set to produce all cuboids. However, such an approach would be both tedious to program and immensely inefficient, given the obvious inter-relationships between the 


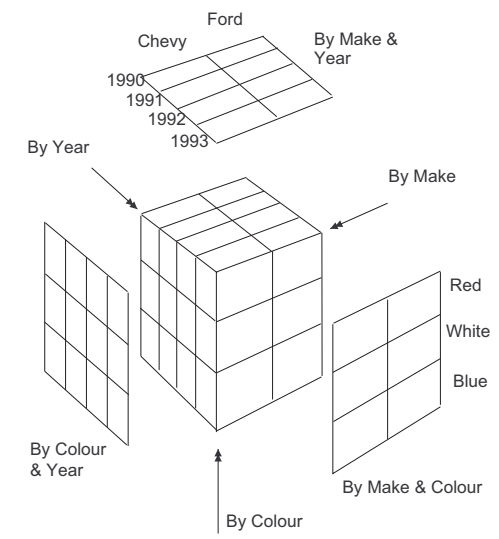

Figure 2.6: A three dimensional data cube for automobile sales data.

various views. Consequently, in 1995, the data cube operator (an SQL syntactical extension) was proposed by Gray et al. [57] as a means of simplifying the process of data cube construction. The main methods for construction of data cubes can be divided into two major classes: top-down and bottom-up approaches. In the former case, we first compute the parent cuboids and then utilize these aggregated views to efficiently compute children. In this case, the goal is to generate coarse granularity tables from views that have previously been aggregated at a finer level of granularity. In contrast, bottom-up computation seeks to first partition the data set on single attributes. Within each partition, we recursively aggregate at finer levels of granularity until we reach the point where no more aggregation is possible/necessary. Bottom-up algorithms tend to favor views with a larger number of dimensions.

\subsubsection{Real-time OLAP}

Most of the traditional OLAP research, and most of the commercial systems, follow the static data cube approach proposed by Gray et al. [57] and materialize all or a subset of the cuboids of the data cube in order to ensure adequate query performance. Building the data cube can be a massive computational task, and significant research has been published on sequential and parallel data cube construction methods [35, 37, 38, 36, 39, 40, 33, 34].

However, the traditional static data cube approach has several disadvantages. The 
OLAP system can only be updated periodically and in batches, e.g. once every week. Hence, latest information can not be included in the decision support process. The static data cube also requires massive amounts of memory space and leads to a duplicate data repository that is separate from the online transaction processing (OLTP) system of the organization. Recently, several practitioners have called for an integrated OLAP/OLTP approach with a real-time OLAP system that is updated instantaneously as new data arrives and always provides an up-to-date data warehouse for the decision support process. However, building such integrated system is not trivial. One major obstacle for any solution to this problem is the significant performance issue with large scale data warehouses. We will further discuss this issue in the next chapter, Section 3.4 and report recent studies to solve this problem.

\subsection{Multi-dimensional Data Indexing}

Multi-dimensional data indexing structures are data structures that aim at efficient storage and retrieval of multi-dimensional data. Multi-dimensional data in general refers to the data distributed in a multi-dimensional space. A multi-dimensional data indexing structure exploits methods to effectively store the existing or arriving data, and at the same time it provides capabilities to search, query, and update stored data. A multi-dimensional data indexing structure is considered real-time if data storage and retrieval can be performed simultaneously in the data structure. In addition, a multi-dimensional data indexing structure is considered parallel if it allows concurrent operations such as search, query, insert, delete, and update in the structure. Multi-dimensional data indexing structures usually implement a tree-like data structure hence called multi-dimensional trees. The logic behind this is that trees provide efficient partitioning, branching, and pruning abilities for tree traversers. Examples of such data structures are R-tree [60] and its variants including R+-tree [100], $\mathrm{R} *$-tree [23], and $\mathrm{X}$-tree [25]. Considering a $d$-dimensional space, $\mathrm{R}$-tree divides the space into hyper-rectangles called Minimum Bounding Rectangles (MBR) with $d$ or a smaller number of dimensions. As an R-tree branches out level by level from the root toward the leaves, these hyper-rectangles cover smaller sub-spaces. The root represents the whole space, and each sub-tree represents a sub-space with $d$ or smaller number of dimension(s). The branching allows the traversers to only take included subtrees into account for a given 
query, and prune subtrees that are not included. The decision on including or excluding a subtree is made using directory nodes that build internal nodes of the tree. A directory node contains the MBR of the subtree (or the sub-space) rooted at the directory node. Leaf nodes of R-tree are data nodes containing real data items available in the $d$-dimensional space. Tree operations include search, query, insert, update, and delete. Also there are other operations such as node split, MBR update, etc. for purposes such as tree maintaining and ensuring of a balanced tree.

One deficiency of R-trees is high overlaps between directory nodes, particularly in high dimensional spaces. There are studies that introduced variants of R-tree to improve on this problem. Minimizing the overlap and coverage for R-tree nodes is crucial; however it was improved in R+-tree [100] and $\mathrm{R}^{*}$-tree [23]. In another method known as X-tree [25], a new type of node called super node was added to the tree. The super node introduces sequential search in its child nodes. As shown in some cases, this method can be more efficient than exploring many overlapping subtrees rooted at the super node.

R-tree [60] and its variants are sequential indexing structures. This means that only one traverser undertaking a tree operation may traverse the tree at a time. After the introduction of R-trees, some studies were performed to parallelize R-trees [73, 107, 75]. In a parallel R-tree, traversers may undertake different tree operations, and traverse the tree to complete the operations at the same time. The parallelization involves locking strategies to prevent false reads and writes by traversers, and the parallelization of tree operations. Kornacker et al. in [21] tried to add extra features to R-tree (such as right-ward links) and to update time-stamps in order to build a parallel R-tree. These features with the provided algorithms for the tree operations enable traversers to perform concurrent tree operations concurrently. In other efforts in $[107,75]$, the parallel R-tree structure proposed by Kornacker, was improved by adding new features. The features included improving the node split operation by soothing its heavy process, reducing unnecessary locks on sibling or parent nodes, and so forth.

R-trees are powerful multi-dimensional data indexing structures that have been employed in applications such as GIS. Their ability to index and store multi-dimensional data can be used to index multi-dimensional databases in decision support systems and to enable parallel and real-time query processing. 


\subsection{Summary}

In this chapter, a comprehensive background of the main subjects in this thesis has been presented. In the next chapters, we focus on the details of the offered solutions to the problems. 


\section{Chapter 3}

\section{Literature Review}

\subsection{Introduction}

In this chapter, we review the literature related to the three subjects of this thesis, and provide brief notes on the studies in these areas.

\subsection{Parallel Sorting on Multi/Many-core Platforms}

Sorting algorithms for GPUs started to appear a few years ago and have been highly competitive so far. Early results include GPUTeraSort [54] based on bitonic merge, and Adaptive Bitonic Sort [58] based on a method by Bilardi et.al. [27]. Hybrid Sort [104] used a combination of bucket sort and merge sort, and D. Cederman et.al. [29] proposed a quick sort based method for GPUs. Both methods [104, 29] suffer from load balancing problems. Until recently, the comparison-based THRUST MERGE method [98] by Nadathur Satish, Mark Harris and Michael Garland of Nvidia corporation was considered the best sorting method for GPUs. THRUST MERGE uses a combination of odd-even merge and two-way merge, and overcomes the load balancing problems. Satish et.al. [98] also presented an even faster GPU radix sort method for the special case of integer sorting.

Other recent studies include [113, 46, 20, 116, 103]. GPU Count Sort [113] proposed a counting sort implementation on GPUs, though it did not provide a comparison of results with other GPU sorting algorithms. The method was only compared with its CPU counterpart. Duane G. Merrill et al. presented a radix sort method [46] and compared it with the CUDPP library [55] implementation, and reported a faster sorting time. Another radix sort approach known as the optimized radix sort [20], emphasizes tuning algorithmic parameters and architectural factors on different Nvidia GPUs. They reported that using their optimizations, optimized radix sort outperforms the CUDPP library [55] and the radix sort presented in [98]. In a comparison-based sorting method by S. Chen et al. 
[103], authors claimed a more practical method compared to previously proposed methods. Their method provides practical adaptability for sorting integers, floats, and structures. As claimed by the authors, their method outperformed GPU quick sort method and a GPU radix sort method, though they have not mentioned the reference of the radix sort method. Another comparison-based GPU sorting method called GPU Warpsort in [116] proposed a merge-sort implementation on GPUs. The authors compared their results with [98] and [29] and reported a higher performance of $30 \%$.

[65] introduced an in-place GPU sort based on an optimized version of bitonic sort. Bitonic sort is simpler (smaller constant time factors) but requires an $O\left(n \log ^{2} n\right)$ work. As observed in [65], their bitonic sort based method outperforms sample sort based methods for small data sets; however their own experiments (Figure 8 in [65]) show that by increasing data size this advantage is lost, with a break even point at approximately 64 million data items.

Yet, a recent paper by Nikolaj Leischner, Vitaly Osipov and Peter Sanders [81] presented a randomized sample sort method for GPUs (GPU SAMPLE SORT) that has shown to significantly outperform THRUST MERGE [98]. However, the fact that GPU SAMPLE SORT is a randomized method implies that its performance can vary with the distribution of the input data because buckets are created through randomly selected data items. For example, the performance analysis presented in [81] measures the runtime of GPU SAMPLE SORT for several input data distributions to document the performance variations observed for different input distributions.

\subsection{Parallel Building of Static Data Cubes on Multi-core Platforms}

In this section, we present a review of the related studies and methods for building static data cubes. Since the second subject in this thesis focuses on the parallel building of static data cubes, we review previous studies in two sections. First, we review the traditional methods used for building static data cubes. These methods are mostly old methods from the past; however, we briefly review them for the completeness of this thesis. Next, we review recent studies on parallel building of static data cubes on multi-core platforms. At last, since we apply a new parallel external sorting method inside the proposed $M C M D$ $C U B E$ method, we provide a survey of the recent external sorting methods and libraries for 
multi-core processors.

\subsubsection{Building Static Data Cubes}

In 1995, the data cube operator (an SQL syntactical extension) was proposed by Gray et al. [56] as a means of simplifying the process of data cube construction. Subsequent to the publication of the seminal data cube paper, a number of independent research projects began to focus on designing efficient algorithms for the computation of the data cube [14, $26,63,77,78,90,92,96,101,106,112,117,121]$.

The algorithms can be divided into three major classes: top-down and bottom-up approaches, and array-based method. In the top-down method, we first compute the parent cuboids and then utilize these aggregated views to efficiently compute the children. Various techniques have been employed for this purpose, including those based on sorting, hashing, and the manipulation of in-memory arrays [14, 96]. Sarawagi et al. in [96] used a top-down approach and divided the lattice of the data cube into chains of cuboids/group-bys. Each chain was then constructed by applying a pipe sort method. In all cases, the goal was to generate coarse granularity tables from views that have previously been aggregated at a finer level of granularity.

In contrast, the bottom-up computation seeks to first partition the data set on single attributes $[26,90]$. Within each partition, we recursively aggregate to the finer levels of granularity; this stops when we reach the point where no more aggregation is possible/necessary. Bottom-up algorithms tend to favor views with a larger number of dimensions. K. Beyer in [26] proposed Iceberg Cubes as a data cube containing only the cells that meet an aggregate condition. Such an aggregate condition is called minsup in [90]. In Iceberg Cubes, a subset of the complete data cube is stored. The subset reflects the valuable cells that play an important role in making decisions in an organization. The conditions or criteria to prune invaluable cells can be set by the organization. Since Iceberg Cube is a partial cube, it can save computation time and storage. In a general classification, top-down and bottomup methods belong to a class of a data model called ROLAP in which data is represented using relational tables.

The last method is the array-based method which belongs to the MOLAP data model where data is stored in the cells of a sparse multi-dimensional array. In the array-based 
method, a $d$-dimensional data cube can be stored in a $d$-dimensional array. Each cell in the array stores one measure value. In this model, the position of a value in the array reveals important information about the value. [121] proposed an array-based method for building data cubes. One disadvantage of this method is the scarcity of the $d$-dimensional array since data cubes are usually sparse and most of their cells represent null values.

\subsubsection{Parallel Building of Static Data Cubes on Multi-core Platforms}

Many parallel methods for building data cubes have been proposed in the literature. Most of these parallel methods build static data cubes on shared-nothing parallel platforms particularly on clusters $[35,37,38,36,39,40,33,34]$. Such methods partition the data cube into smaller groups of sub-cubes and distribute them between cluster nodes. Each node then builds a sub-cube of the complete data cube. Recently, a few parallel methods appeared for building static data cubes on multi-core platforms. [84] presented an improvement on the CPU and cache behavior with providing a cube partitioning method called CC-Cubing. Their method is an improvement of another bottom-up method called Star-Cubing [117] for building static data cubes. They compared their results with those of $[90,117]$ and another bottom-up method called MM-Cubing [101]. It is reported that parallel CC-Cubing, implemented using multi-threading, provided a performance improvement of $27 \%$ compared to the sequential version.

Another study reported the implementation of partial data cubes on multi-core processors though they reported some slow-down due to the disk I/O latency [67]. [120] introduced a cube partitioning approach called Paracube to distribute tuples of the fact table between multiple servers that can be merged to answer queries. Their experiments were performed on multi-core CPUs. In another study for building data cubes, CC-BUC method [59] was proposed to build data cubes on multi-core CPUs and GPUs. CC-BUC is a bottom-up method combined with breadth-first strategies. CC-BUC also tries to provide cache-concise strategies to minimize the CPU cache misses. The method was tested on both multi-core CPUs and GPUs and showed a speedup of six times faster than the bottomup method in [90]. In an array-based method [68], authors proposed a multi-dimensional array that can be extended in any dimension without the need to relocate existing data. Authors implemented the method on a SUN Enterprise machine with 24 cores and reported 
a close to linear speedup with load balancing. [66] implemented a multi-core version of the Closed Cube method [118] which is a variation of the Star-Cubing method [117]. The method applies a cost-based load balancing strategy to handle data distribution and skew on multi-core processors.

\subsubsection{Parallel External Sorting on Multi-core Platforms}

External sorting, also called out-of-core sorting, refers to the sorting of data items or records that reside in external storage. External sorting is a fundamental problem in computer science and has been extensively studied in the literature. Although there is a considerable number of parallel external sorting algorithms for traditional parallel platforms (such as clusters), there have been only a few of them for multi-core platform [9, 8, 42, 22]. In order to use old parallel external sorting algorithms on the new parallel platforms, one needs to adapt and tune them for the architecture of the new platforms (e.g. multi-core platform).

Among the recent external sorting implementations, STXXL library [42] showed a high performance and can be used for multi-core processors. STXXL [42] is a standard sorting library including external memory algorithms. The core of the STXXL sorting is a merge sort algorithm combined with a pipe-lined I/O strategy. It was originally designed for single processor platforms; however it was then adapted to work for clusters and later multi-core processors through combination with MCSTL library [4]. MCSTL library is a recent standard library for multi-core platforms working on in-memory data.

Other external sorting libraries include TPIE [76] and LEDA-SM [79] and [110]. However, none of these libraries were adapted yet for multi-core processors.

\subsection{Real-time OLAP}

There are few studies in the literature for an integrated OLAP/OLTP approach that supports Real-time OLAP. Some recent studies have tried to address this problem by providing "quasi real-time" incremental maintenance schemes and loading procedures for static data cubes (e.g. [28, 69, 94, 95]). However, these approaches are not fully real-time. Building a parallel OLAP server is considerably more complex than concurrent updates and searches in general databases which have been studied since the 90's, e.g. in [21, 31], and more recently in $[64,80]$. Concurrent operations in spatial databases have recently been studied 
e.g. in [70]. However, the general DB index structures presented are not efficient for the large number of dimensions typically required for OLAP. Another important difference is the elaborate dimension hierarchies which are typical for OLAP systems. To our knowledge, the only published fully dynamic/real-time data structure for OLAP queries on data cubes is the $D C$-tree introduced by Kriegel et al. [47], which is a sequential tree-based indexing structure specifically designed for data warehouses with dimension hierarchies. Even though DC-tree was published more than 10 years ago, and despite the fact that it does provide an algorithmic solution for Real-time OLAP systems, it has not found its way into commercial OLAP systems. The DC-tree in terms of its indexing structure is an extension of the classical X-tree [25] and R-tree [60] data structures for multi-dimensional data indexing. For a discussion of R-tree and X-tree, please refer to Chapter 2, Section 2.4. The DC-tree inherits the tree structure presented in the R-tree and its variants in particular $\mathrm{X}$-tree. At the heart of any R-tree and consequently the DC-tree, there are directory nodes that direct tree traversers. In general, there are three types of nodes in a DC-tree. Data nodes which are leaves of the tree representing tuples of the fact table of data warehouse. Directory nodes that are internal nodes of the tree, and super nodes, which are similar to directory nodes, with the difference of unlimited capacity to store child nodes. The DCtree also benefits from partial ordering generated for attribute values of each dimension of a data warehouse. This partial ordering is generated using a conceptual hierarchy for each dimension that groups and stores attribute values of each dimension. Figure 3.1 shows an example of a hierarchy schema and conceptual hierarchy for a sample customer dimension. The DC-tree is built gradually by inserting new data nodes and also new directory nodes wherever it is necessary. We refer readers to [47] for a detailed discussion.

In addition to the DC-tree, there have been other efforts to build a Real-time OLAP system. [91] presented a data cube method partitioned by the dimensions of the data cube. Each partition is sorted and a cube tree is built for the partition. A cube tree is built based on emphPacked R-tree method proposed in [93]. The main disadvantage of this method is that new data needs to be sorted, partitioned and bulk-loaded into the cube trees. This drawback disables real-time responses and such a method may not be considered completely real-time. [71] proposed a method called MVCC-DW to overcome the problem of data cube maintenance and batch updates. The method tries to provide multiple versions for an 


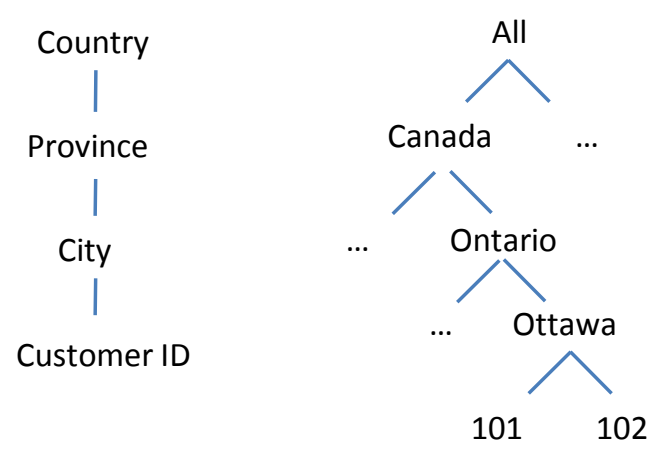

Figure 3.1: Hierarchy schema(left) and conceptual hierarchy(right) for dimension Customer.

array-based data cube (MOLAP); however the method suffers from the overlap between the multiple versions and has to update them frequently. In other efforts, [106, 105, 78] compression methods were proposed to summarize the data cube and reduce the cost of building data cubes. [105] tried to integrate a hierarchical clustering with the Dwarf method [106] to speedup OLAP transactions. However, these methods still try to materialize the data cube and suffer from the cost of building the data cube and bulk loads. OLAP operations such as roll-up and drill-down are also very costly [105] in these methods.

Doka in [43] presented an online querying system called HIS with concept hierarchies. Concept hierarchies were also used in the DC-tree[47]; however, HIS lacks an R-tree data cube structure similar to the DC-tree. HIS was designed for peer-to-peer systems. Asiki et al. in [17] presented a similar method that uses concept hierarchies; however, similar to [43], it does not contain a centralized tree structure. In another study by Asiki et al. in $[18,19]$, authors improved their previous work and implemented a decentralized concept hierarchy on a grid system. The new method is similar to the DC-tree and is able to perform updates and lookups simultaneously.

In another study by Doka et al. [45], an online system was presented that is capable of performing online OLAP operations. Their method is based on concept hierarchies similar to $[17,18,19]$; however, the method lacks a multi-dimensional data indexing structure such as R-tree. The method tries to integrate the concept hierarchies of different dimensions and use them to store input data. This is in contrast to the DC-tree method in which input data is stored in a single tree-like data structure. The most recent work by Doka et al.[44] 
called Brown Dwarf which tries to build a distributed version of the originally centralized Dwarf method [106] in peer-to-peer networks. The method is parallelized over the network and provides online update and query transactions. A similar work based on the DC-tree is presented in [15] which emphasizes on the temporal features of data warehouse. The method is called TiC-tree and provides extensions to the DC-tree to support temporal data warehouses.

In spite of the recent efforts, parallelization of Real-time OLAP on multi/many-core processors has not been addressed yet. However, as referred above, there have been few methods $([18,19,45,44])$ that parallelize Real-time OLAP for other parallel platforms such as peer-to-peer and grid systems. Yet, cloud platforms open a new area for parallelization of Real-time OLAP.

Apart from data warehousing methods, there have been a few studies for providing indexing structures on cloud systems. These efforts were usually for general data indexing in 1-dimensional and multi-dimensional spaces. B-trees and its variants are usually considered for 1-dimensional data indexing on cloud [115, 52, 114], while for multi-dimensional data indexing, R-trees are applied [119, 111]. In [111], global and local R-trees were used to index multi-dimensional data in a cloud environment. However, the method does not support hierarchical data present in OLAP and data warehousing systems. A similar multidimensional index was proposed in [119]. [114] introduced a B-tree approach for cloud platforms; however the method only supports 1-dimensional index and may not be used for multi-dimensional data warehouses. [74] proposed a fault-tolerant distributed method for query processing. The method is built on top of R-trees and supports regular range queries. Similarly, this method does not address the OLAP and data warehousing issues related to dimension hierarchies.

\subsubsection{Real-time OLAP and Big Data Platforms}

The fast growth of data size in computing industries demands novel methods for data representation, indexing, storage and retrieval, and methods for parallelization of these tasks. Hadoop [3] and its file system, HDFS, is an example of such systems aiming at fast storage and retrieval of large data volumes so called Big Data. Although Hadoop is efficient to handle large data, it is suited for batch processing and is not designed for real-time data 
processing. Many efforts including Hive [108], HadoopDB [13] and other variants tried to enable data warehousing in Hadoop. However, they do not provide "real-time" OLAP.

Other efforts include systems and platforms from Google such as BigTable [32], BigQuery [2], and Dremel [86], all based on Google File System (GFS). BigTable was introduced as a DBMS for large data sizes specifically designed for Google applications. BigQuery, built on top of BigTable, was introduced to support fast query answering in large databases. Dremel uses a columnar data representation scheme and provides data warehousing and querying support for read-only data. The main drawback of the mentioned systems is again their disability in supporting real-time updates.

As another industrial effort, Storm project [10] introduced a distributed computing model that is applied in Twitter. It is proposed as a solution to overcome the limits of batch processing in Hadoop-based systems and to provide real-time data processing. The model uses computational resources that process in-flight data. It assumes that the data can quickly migrate to different resources so called Bolts. However this is not the case in large and distributed real-time data warehouses where the data is stored in large chunks distributed over cloud nodes. In such cases, it is not feasible to consistently migrate data to different nodes for processing. Instead, it is more efficient to move the computation power to where the requested data resides.

\subsection{Summary}

In this chapter, we reviewed related projects in the three subjects of this thesis. In the next chapters, detailed discussions of the offered methods will be presented along with the experiments and achieved results. 


\section{Chapter 4}

\section{GPU BUCKET Sort}

\subsection{Introduction}

In this chapter, we propose a GPU sorting method called GPU Bucket Sort for Nvidia GPUs. We discuss the algorithmic steps and details of the GPU Bucket Sort method and compare it with other state-of-the-art GPU sorting methods at the time of development. First, we outline the method and its sorting algorithm. Along with the algorithm, we discuss the technical details of our CUDA implementation on Nvidia GPUs. We conclude this chapter with an evaluation of experiments that confirm the advantage of the GPU Bucket Sort method over other sorting methods. For details of Nvidia GPU architecture and other introductory material on GPU sorting, please refer to Chapter 2, Section 2.2.1, and Chapter 1 , respectively.

\subsubsection{Statement of the Problem}

We phrase the addressed problem in this chapter as follows:

Given a many-core Graphics Processor such as Nvidia GPUs, we seek a parallel sorting method for Nvidia GPUs, able to sort a list of unsorted data items residing in the global memory of the graphics processor. The sorting method is expected to be comparison-based and able to sort data items using real-value and integer keys.

\subsection{GPU Bucket Sort: Deterministic Sample Sort For GPUs}

In this section we outline GPU Bucket Sort, a deterministic sample sort algorithm for GPUs, and discuss our CUDA implementation of GPU Bucket Sort. An overview of GPU Bucket Sort is shown in Algorithm 1. GPU Bucket Sort consists of a local sort (Step 1), a selection of samples that define balanced buckets (Steps 3-5), moving all data into those buckets (Steps 6-8), and a final sort of each bucket (Step 9). In our implementation of GPU Bucket 
Sort we introduced several adaptations to the structure of GPUs, in particular the two level memory hierarchy, the large difference in memory access times between those two levels, and the small size of the local shared memories. We experimented with several bucket sizes and number of samples in order to best fit them to the GPU memory structure. For sorting the selected sample and the bottom level sorts of the individual buckets, we experimented with several existing GPU sorting methods such as bitonic sort, adaptive bitonic sort [58] based on [27], and parallel quick sort [30].

The following discussion of our implementation of GPU Bucket Sort will focus on GPU performance issues related to shared memory usage, coalesced global memory accesses, and avoidance of conditional branching.

\subsection{Algorithm}

Consider an input array $A$ with $n$ data items in global memory and a typical local shared memory of size $\frac{n}{m}$ data items.

In Steps 1 and 2 of Algorithm 1, we split the array $A$ into $m$ sublists of $\frac{n}{m}$ data items each and then locally sort each of those $m$ sublists. More precisely, we create $m$ thread blocks of 512 threads each, where each thread block sorts one sublist using one SM. Each thread block first loads a sublist into the SM's local shared memory using a coalesced parallel read from global memory. Note that, each of the 512 threads is responsible for $\frac{n}{m} / 512$ data items. The thread block then sorts a sublist of $\frac{n}{m}$ data items in the SM's local shared memory. We tested different implementations for the local shared memory sort within an SM, including quick sort[30], bitonic sort, and adaptive bitonic sort [27]. In our experiments, bitonic sort was consistently the fastest method, despite the fact that it requires $O\left(n \log ^{2} n\right)$ work. The reason is that, for Step 2 of Algorithm 1, we always sort a small fixed number of data items, independent of $n$ (about $2 K$ for the GTX 2XX/Tesla series and $6 K$ for the Fermi). For such a small number of items, the simplicity of bitonic sort, its small constants in the running time, and its perfect match for SIMD style parallelism outweigh the disadvantage of additional work.

In Step 3 of Algorithm 1, we select $s$ equidistant samples from each sorted sublist. (The implementation of Step 3 is built directly into the final phase of Step 2 when the sorted sublists are written back into global memory.) 
Algorithm 1: GPU Bucket Sort (Deterministic Sample Sort For GPUs)

Input: An array $A$ with $n$ data items stored in global memory.

Output: Array $A$ sorted.

Split the array $A$ into $m$ sublists $A_{1}, \ldots, A_{m}$ containing $\frac{n}{m}$ items each where $\frac{n}{m}$ is the shared memory size at each SM.

Local Sort: Sort each sublist $A_{i}(i=1, \ldots, m)$ locally on one SM, using the SM's shared memory as a cache.

Local Sampling: Select $s$ equidistant samples from each sorted sublist $A_{i}(i=1, \ldots, m)$ for a total of $s m$ samples.

Sorting All Samples: Sort all sm samples in global memory, using all available SMs in parallel.

Global Sampling: Select $s$ equidistant samples from the sorted list of $s m$ samples. We will refer to these $s$ samples as global samples.

Sample Indexing: For each sorted sublist $A_{i}(i=1, \ldots, m)$ determine the location of each of the $s$ global samples in $A_{i}$. This operation is done for each $A_{i}$ locally on one SM, using the SM's shared memory, and will create for each $A_{i}$ a partitioning into $s$ buckets $A_{i 1}, \ldots, A_{i s}$ of size $a_{i 1}, \ldots, a_{i s}$.

Prefix Sum: Through a parallel prefix sum operation on $a_{11}, \ldots, a_{m 1}, a_{12}, \ldots, a_{m 2}, \ldots, a_{1 s}, \ldots, a_{m s}$ calculate for each bucket $A_{i j}(1 \leq i \leq m, 1 \leq j \leq s)$ its starting location $l_{i j}$ in the final sorted sequence.

Data Relocation: Move all $s m$ buckets $A_{i j}(1 \leq i \leq m, 1 \leq j \leq s)$ to location $l_{i j}$. The newly created array consists of $s$ sublists $B_{1}, \ldots, B_{s}$ where $B_{j}=A_{1 j} \cup A_{2 j} \cup \ldots \cup A_{m j}$ for $1 \leq j \leq s$. Sublist Sort: Sort all sublists $B_{j}, 1 \leq j \leq s$, using all SMs. 
Note that, the sample size $s$ is a free parameter that needs to be tuned. With increasing $s$, the sizes of buckets created in Step 8 decrease and the time for sorting those buckets (Step 9) decreases as well. However, the time for managing the buckets (Steps 3-7) grows with increasing $s$. This trade-off will be studied in Section 4.4 of this chapter where we show that $s=64$ provides the best performance. In Step 4, we sort all $s m$ selected samples in global memory, using all available SMs in parallel. Here, we compared GPU bitonic sort [54], adaptive bitonic sort [58] based on [27], and gpu quick sort[30]. Our experiments indicate that for up to 16 million data items, simple bitonic sort is still faster than others due to its simplicity, small constants, and complete avoidance of conditional branching. Hence, Step 4 was implemented via bitonic sort. In Step 5, we again select $s$ equidistant global samples from the sorted list of $s m$ samples. Here, each thread block/SM loads the $s$ global samples into its local shared memory where they will remain for the next step.

In Step 6, we determine for each sorted sublist $A_{i}(i=1, \ldots, m)$ of $\frac{n}{m}$ data items the location of each of the $s$ global samples in $A_{i}$. For each $A_{i}$, this operation is done locally by one thread block on one SM, using the SM's shared memory, and will create for each $A_{i}$ a partitioning into $s$ buckets $A_{i 1}, \ldots, A_{i s}$ of size $a_{i 1}, \ldots, a_{i s}$. Here, we apply a parallel binary search algorithm to locate the global samples in $A_{i}$. More precisely, we first take the $\frac{S}{2}$-th global sample element and use one thread to perform a binary search in $A_{i}$, resulting in a location $l_{s / 2}$ in $A_{i}$. Then we use two threads to perform two binary searches in parallel, one for the $\frac{s}{4}$-th global sample element in the part of $A_{i}$ to the left of location $l_{s / 2}$, and one for the $\frac{3 s}{4}$-th global sample element in the part of $A_{i}$ to the right of location $l_{s / 2}$. This process is iterated $\log s$ times until all $s$ global samples are located in $A_{i}$. With this, each $A_{i}$ is split into $s$ buckets $A_{i 1}, \ldots, A_{i s}$ of size $a_{i 1}, \ldots, a_{i s}$. Note that, we do not simply perform all $s$ binary searches fully in parallel in order to avoid memory contention within the local shared memory [6].

Step 7 uses a prefix sum calculation to obtain for all buckets their starting location in the final sorted sequence. The operation is illustrated in Figure 4.1 and can be implemented with coalesced memory accesses in global memory. Each row in Figure 4.1 shows the $a_{i 1}, \ldots, a_{i s}$ calculated for each sublist. The prefix sum is implemented via a parallel column sum (using all SMs), followed by a prefix sum on the columns sums (on one SM in local shared memory), and a final update of the partial sums in each column (using all SMs). 


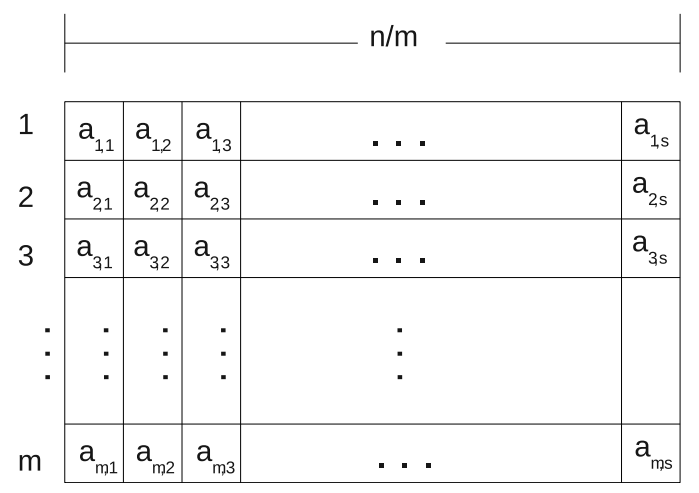

Figure 4.1: Illustration Of Step 7 In Algorithm 1

In Step 8, the $s m$ buckets are moved to their correct location in the final sorted sequence. This operation is perfectly suited for a GPU and requires one parallel coalesced data read followed by one parallel coalesced data write operation. The newly created array consists of $s$ sublists $B_{1}, \ldots, B_{s}$ where each $B_{j}=A_{1 j} \cup A_{2 j} \cup \ldots \cup A_{m j}$ has at most $\frac{2 n}{s}$ data items [102]. In Step 9, we sort each $B_{j}$ using the same bitonic sort implementation as in Step 4. We observed that for our choice of (see Section 4.4 below), each $B_{j}$ contains at most $4 M$ data items. For such small data sets, simple bitonic sort is again the fastest sorting algorithm for each $B_{j}$ due to bitonic sort's simplicity, small constants, and complete avoidance of conditional branching.

\subsection{Experimental Results}

In this section, we first prepare a test setup to perform experiments on the GPU Bucket Sort method. Next, we present the results of the tests.

\subsubsection{Setup}

For our experimental evaluation, we executed Algorithm 1 on five different GPUs (Nvidia Tesla, GTX 285/2GB, GTX 285/1GB, GTX 260, and Fermi GTX 480) for various data sets of different sizes, and compared our results with those reported in [98] and [81] which are the current best GPU sorting methods and outperform previous methods such as e.g. [104]. Unfortunately, we were unable to compare our results with [99] because the authors did not supply us with their code. Therefore, their published performance data is for a different 
Table 4.1: Performance Characteristics For Nvidia Tesla C1060, GTX 285 with 2 GB memory, GTX 285 with 1 GB memory, GTX 260 and Fermi GTX 480 (Source: [7]).

\begin{tabular}{llllll}
\hline \hline GPU & Tesla & GTX 285 & GTX 285 & GTX & Fermi \\
& C1060 & $(2$ GB $)$ & $(1 \mathrm{~GB})$ & 260 & GTX 480 \\
\hline Number of Cores & 240 & 240 & 240 & 216 & 480 \\
\hline Core Clock Rate $(\mathrm{MHz})$ & 602 & 648 & 648 & 576 & 700 \\
\hline Global Memory Size $(\mathrm{GB})$ & 4 & 2 & 1 & 0.896 & 1.5 \\
\hline Memory Clock Rate $(\mathrm{MHz})$ & 1600 & 2322 & 2484 & 1998 & 1848 \\
\hline Memory Bandwidth $(\mathrm{GB} / \mathrm{Sec})$ & 102 & 149 & 159 & 112 & 177 \\
\hline \hline
\end{tabular}

GPU (GTX 280) that we did not have available. It uses 32-bit keys (instead of 64-bit keys used in [98], [81] and our work), and reported their performance only on small data sets up to 64 million data items. Table 4.1 shows some important performance characteristics of the five different GPUs.

\subsubsection{Results}

Figure 4.2 shows a comparison of the running times of our GPU Bucket Sort implementation on the Tesla C1060, GTX 260, GTX 285 (with 2 GB memory) and Fermi GTX 480 for varying number of data items. Each data point shows the average of 100 experiments. The observed variance was less than $1 \mathrm{~ms}$ for all data points since GPU Bucket Sort is deterministic and any fluctuation observed was due to noise on the GPU (e.g. operating system related traffic). All three curves show a growth rate very close to linear which is encouraging for a problem that requires $O(n \log n)$ work. Not surprisingly, GPU Bucket Sort performs best on the Fermi. Interestingly, it performs better on the GTX 285 than both Tesla and GTX 260, and it even performs better on the GTX 260 than on the Tesla C1060. Note that the memory bandwidth for the GTX 260 is higher than for the Tesla C1060. This indicates that GPU Bucket Sort is memory bandwidth bound which is expected for sorting methods since the sorting problem requires only very little computation but a large amount of data movement. For individual steps of GPU Bucket Sort, the order can sometimes be reversed. For example, we observed that Step 2 of Algorithm 1 (local sort) runs faster on the Tesla C1060 than on the GTX 260 since this step is executed locally on each SM. Because this step is compute-bound, its performance is largely determined by the number of 


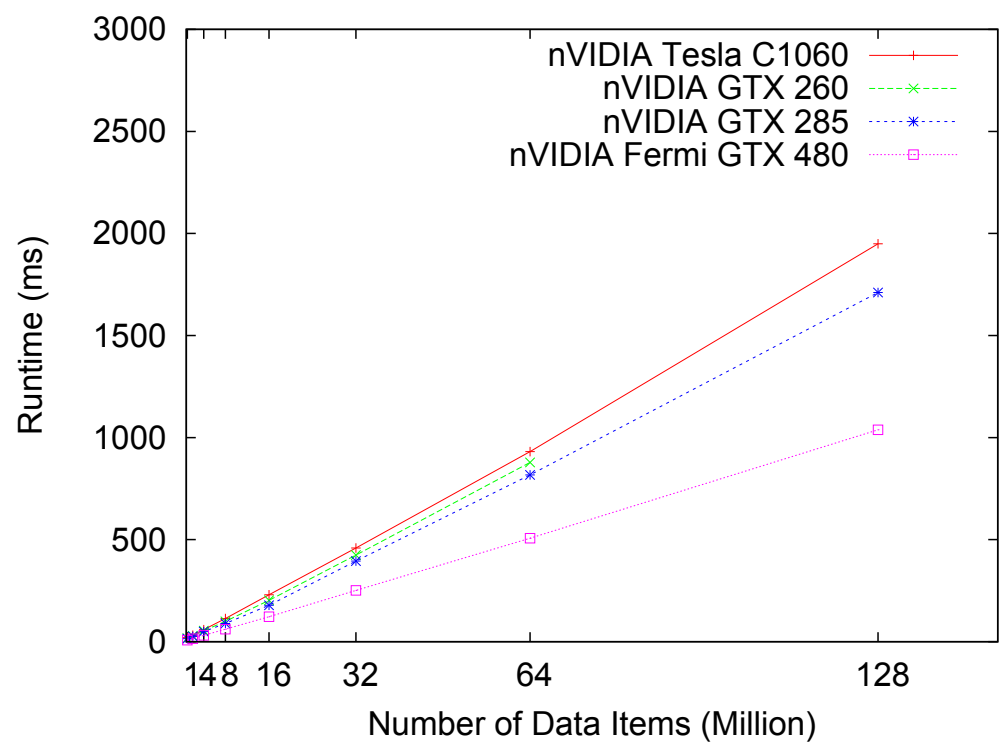

Figure 4.2: Performance Of Deterministic Sample Sort For GPUs (GPU BUCKET SORT). Total runtime for varying number of data items on different GPUs: Nvidia Tesla C1060, GTX 260 and GTX 285.

SMs and the performance of the SM's cores. Step 3 is compute-bound because it is taking samples locally and sorting them. Step 4 needs to sort data in global memory and is therefore memory bandwidth bound. Steps 5, 6, and 7 are all compute-bound steps because they do not need significant data movements to/from global memory. Steps 8 and 9 are memory bandwidth bound because these steps include high loads of data movement and sorting in global memory. In summary, the entire algorithm is memory bandwidth bound because the steps that are taking most of the time are memory bandwidth bound while compute-bound steps contribute only a small fraction of the total time.

Note that the GTX 285 and Fermi GTX 480 remained the fastest machines, even for all individual steps. We note that GPU Bucket Sort can sort up to $n=64 M$ data items within the $896 \mathrm{MB}$ memory available on the GTX 260 (see Figure 4.2). On the GTX 285 with 2 GB memory and Tesla C1060 our GPU Bucket Sort implementation can sort up to $n=256 M$ and $n=512 M$ data items, respectively (see Figures 4.5\&4.6). On the Fermi GTX 480 with $1.5 \mathrm{~GB}$ memory, it is able to sort up to $n=128 M$ data items.

Figure 4.3 shows in detail the time required for the individual steps of Algorithm 1 when executed on a GTX 285 and Fermi GTX 480. We observe that sublist sort (Step 9) and local sort (Step 2) represent the largest portion of the total runtime of GPU Bucket 


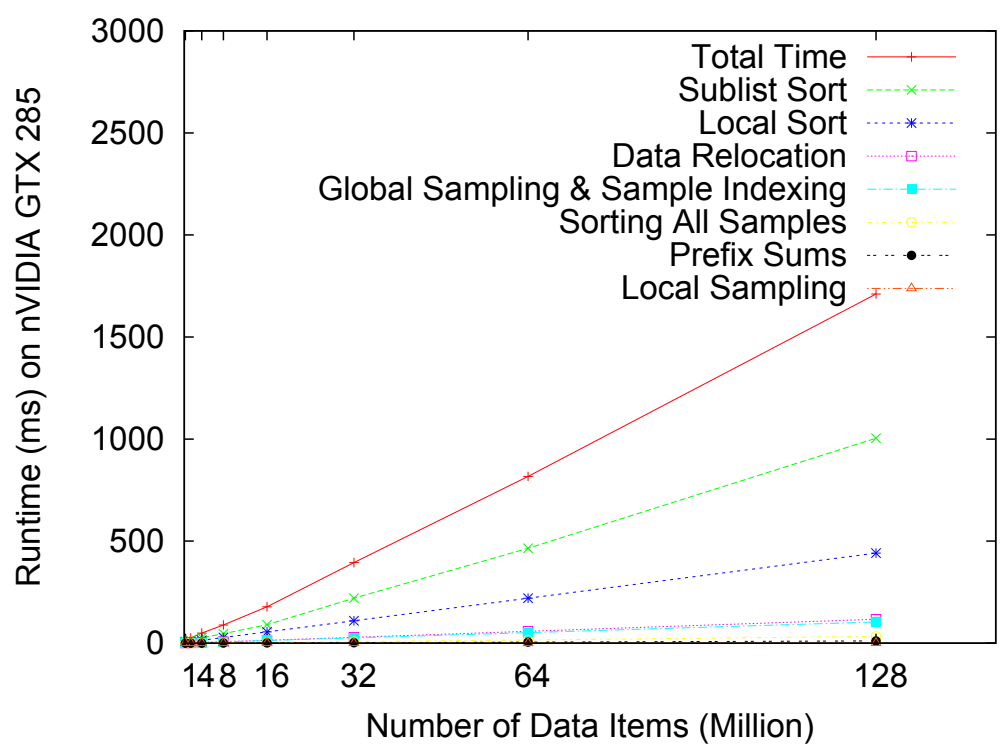

(a)

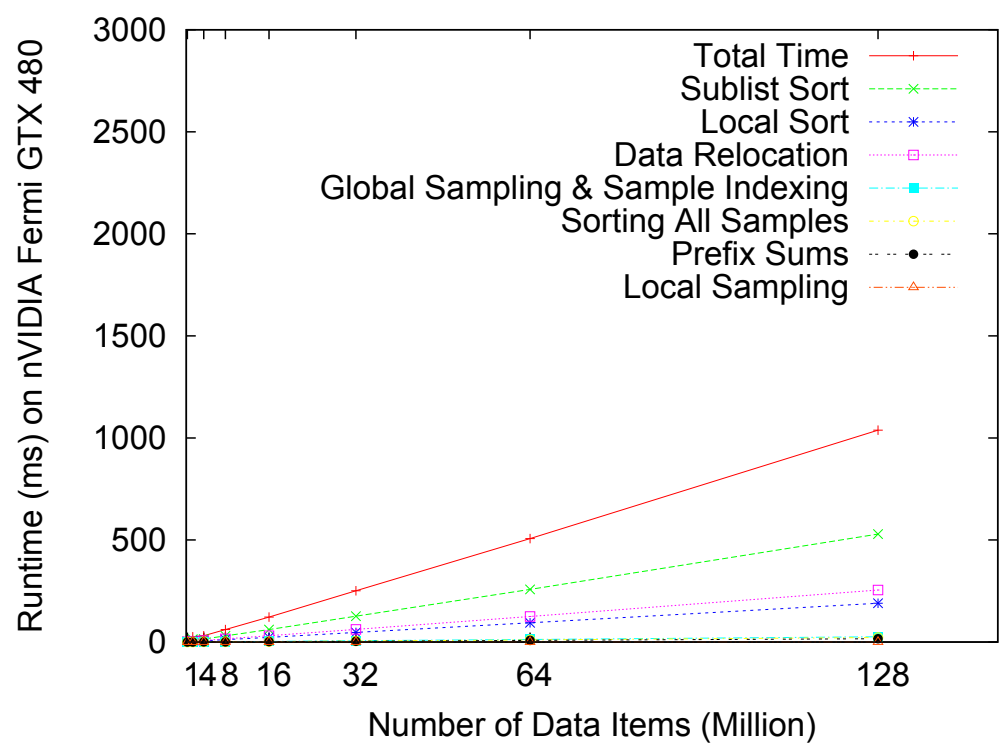

(b)

Figure 4.3: Performance Of Deterministic Sample Sort For GPUs (GPU BUCKET SoRT). Total runtime and runtime for individual steps of Algorithm 1 for varying number of data items. (a) For Nvidia GTX 285 and and (b) for Nvidia Fermi GTX 480. 

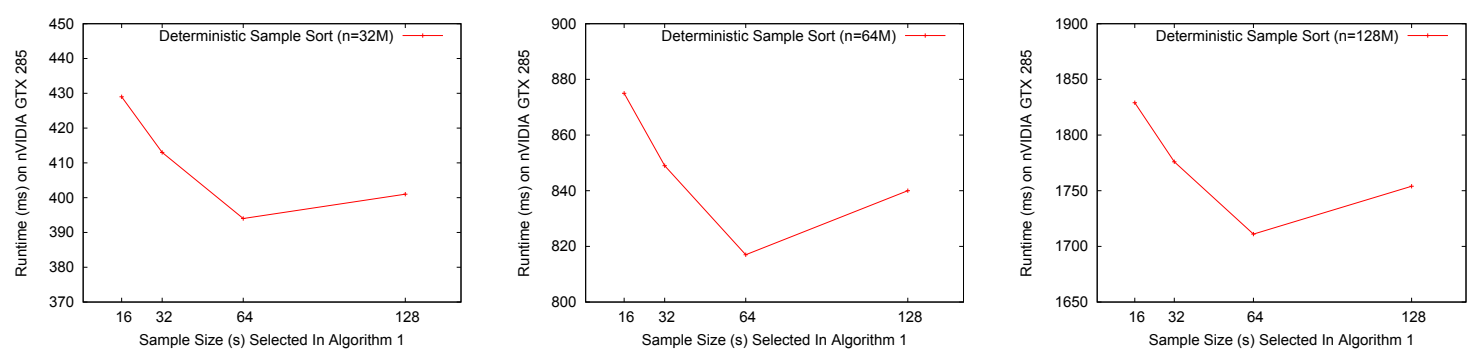

Figure 4.4: Runtime Of Algorithm 1 As A Function Of Selected Sample Size $s$ For Fixed $n=32 M, n=64 M$, and $n=128 M$.

Sort. This is very encouraging in that the "overhead" involved to manage the deterministic sampling and generate buckets of guaranteed size (Steps 3-7) is small. We also observe that the data relocation operation (Step 8) is very efficient and a good example of the GPU's great performance for data parallel access when memory accesses can be coalesced (see Section 2.2.1.2). Note that, the sample size $s$ in Algorithm 1 is a tunable parameter. With increasing $s$, the sizes of sublists $B_{j}$ created in Step 8 of Algorithm 1 decrease and the time for Step 9 decreases as well. However, the time for Steps 3-7 grows with increasing $s$. This trade-off is illustrated in Figure 4.4 which shows the total runtime for Algorithm 1 as a function of $s$ for fixed $n=32 M, 64 M, 128 M$. As shown in Figure 4.4, the total runtime is smallest for $s=64$, which is the parameter value chosen for our GPU Bucket Sort code.

Figures 4.5 and 4.6 show a comparison between GPU Bucket Sort and the best GPU sorting methods at the time of development, randomized GPU SAMPLE SORT [81] and Thrust Merge [98]. Figure 4.5 shows the running times for all three methods on a GTX 285 and Figure 4.6 shows the running times of all three methods on a Tesla C1060. Note that, [98] and [81] did not report runtimes for the GTX 260 and Fermi GTX 480. For GPU Bucket Sort, all running times are the averages of 100 experiments, with less than $1 \mathrm{~ms}$ observed variance. For randomized GPU SAMPLE SORT and ThrUST MERGE, the running times shown are the ones reported in [81] and [98]. For Thrust MERGE, performance data is only available for up to $n=16 M$ data items. For larger values of $n$, the current Thrust MERGE code shows memory errors [53]. As reported in [81], the current randomized GPU SAMPLE SORT code can sort up to $32 M$ data items on a GTX 285 with 1 GB memory and up to $128 M$ data items on a Tesla C1060. Our GPU Bucket Sort implementation appears to be more memory efficient. GPU Bucket Sort can sort up 
to $n=256 M$ data items on a GTX 285 with 2 GB memory and up to $n=512 M$ data items on a Tesla C1060. Therefore, Figures 4.5(a) and 4.6(a) show the performance comparison with higher resolution for up to $n=64 M$ and $n=128 M$, respectively, while Figures 4.5(b) and 4.6(b) show the performance comparison for the entire range up to $n=256 M$ and $n=512 M$, respectively.

We observe in Figures 4.5(a) and 4.6(a) that, as reported in [81], randomized GPU SAMPLE SORT [81] significantly outperforms Thrust Merge [98]. Most importantly, we observe that randomized sample sort (GPU SAMPLE SORT) [81] and our deterministic sample sort (GPU Bucket Sort) show nearly identical performance on both, the GTX 285 and Tesla C1060. Note that, the experiments in [81] used a GTX 285 with 1 GB memory whereas we used a GTX 285 with 2 GB memory. As shown in Figure 4.1, the GTX 285 with 1 GB has a slightly better memory clock rate and memory bandwidth than the GTX 285 with 2 GB which implies that the performance of deterministic sample sort (GPU Bucket Sort) on a GTX 285 is actually a few percent better than the performance of randomized sample sort (GPU SAMPLE SORT).

The data sets used for the performance comparison in Figures 4.5 and 4.6 were uniformly distributed, random data items. The data distribution does not impact the performance of deterministic sample sort (GPU Bucket Sort) but has an impact on the performance of randomized sample sort (GPU SAMPLE SORT). It is notable in Figure 4.6(a) that randomized sample sort shows a slightly better performance versus our deterministic sample sort from 64 million data items to 128 million data times. One can argue that this trend may continue and lead to larger divergences for larger data sets. However, the uniform data distribution used for Figures 4.5 and 4.6 is a best case scenario for randomized sample sort where all bucket sizes are nearly identical. Non-uniform data sets are shown in [81] to have a significant degradation impact on the performance of the randomized sample sort. Figure 4.7 shows that our deterministic sample sort (GPU Bucket Sort) is stable under different types of data distributions. We tested three types of data distribution: uniform, gaussian, and zipf. As seen in the figure, different input data distributions have little influence on the time performance of our algorithm.

Figures 4.5(b) and 4.6(b) show the performance of GPU Bucket Sort for up to $n=256 \mathrm{M}$ and $n=512 M$, respectively. For both architectures, GTX 285 and Tesla C1060, we observe 


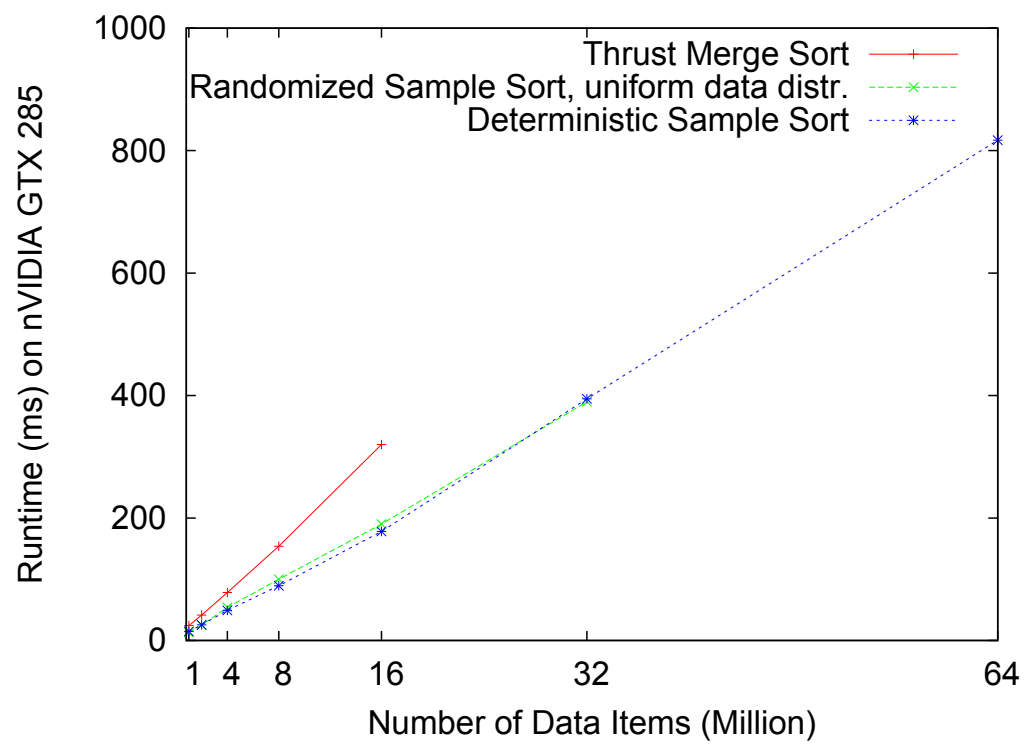

(a) Number of Data Items Up To 64,000,000.

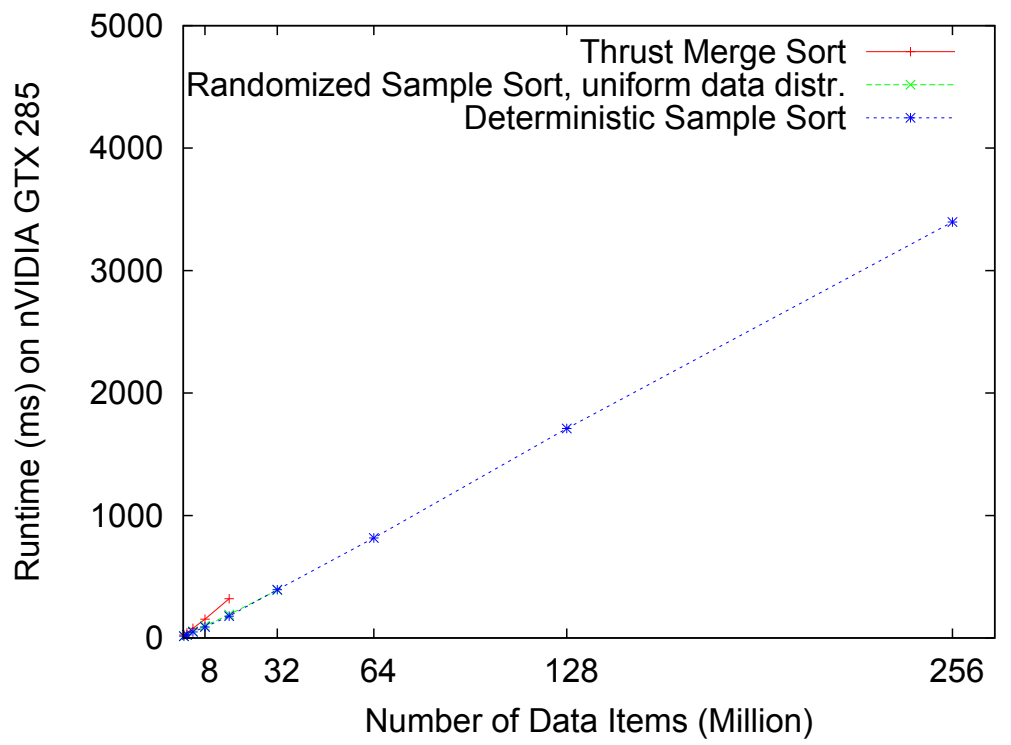

(b) Number of Data Items Up To 256,000,000.

Figure 4.5: Comparison between Deterministic Sample Sort (GPU Bucket Sort), Randomized Sample Sort (GPU S AMPLE SORT) [81] and ThrUST MERGE [98]. Total runtime for varying number of data items on an Nvidia GTX 285. ([98] and [81] provided data only for up to $16 \mathrm{M}$ and $32 \mathrm{M}$ data items, respectively.) 


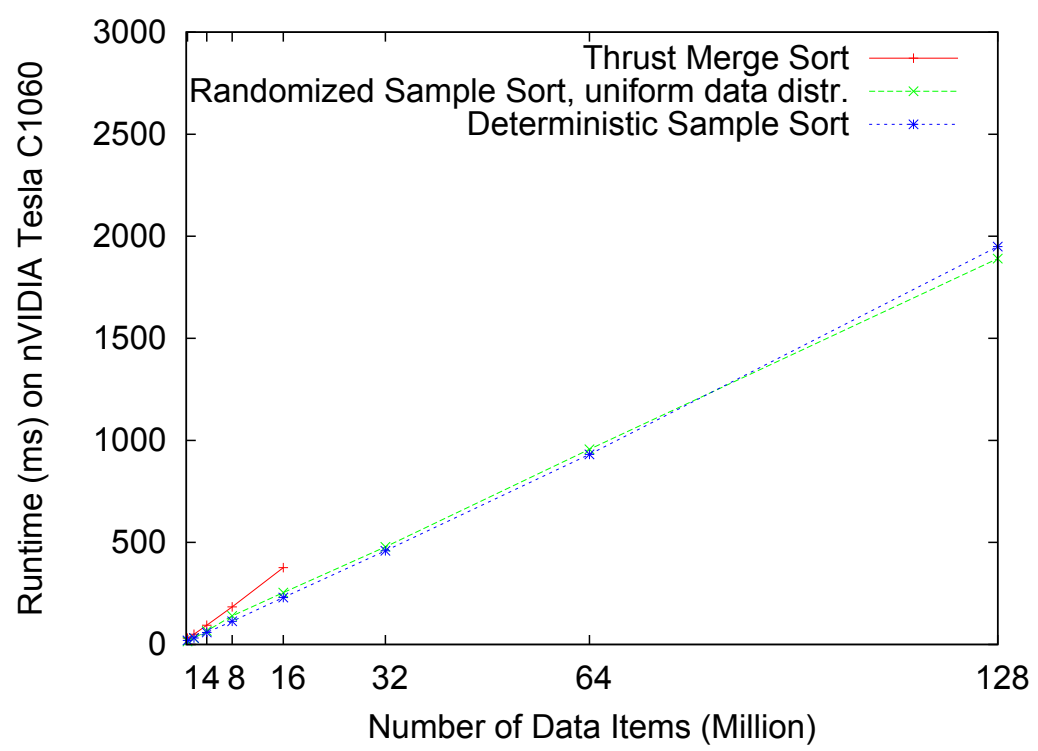

(a) Number of Data Items Up To 128,000,000.

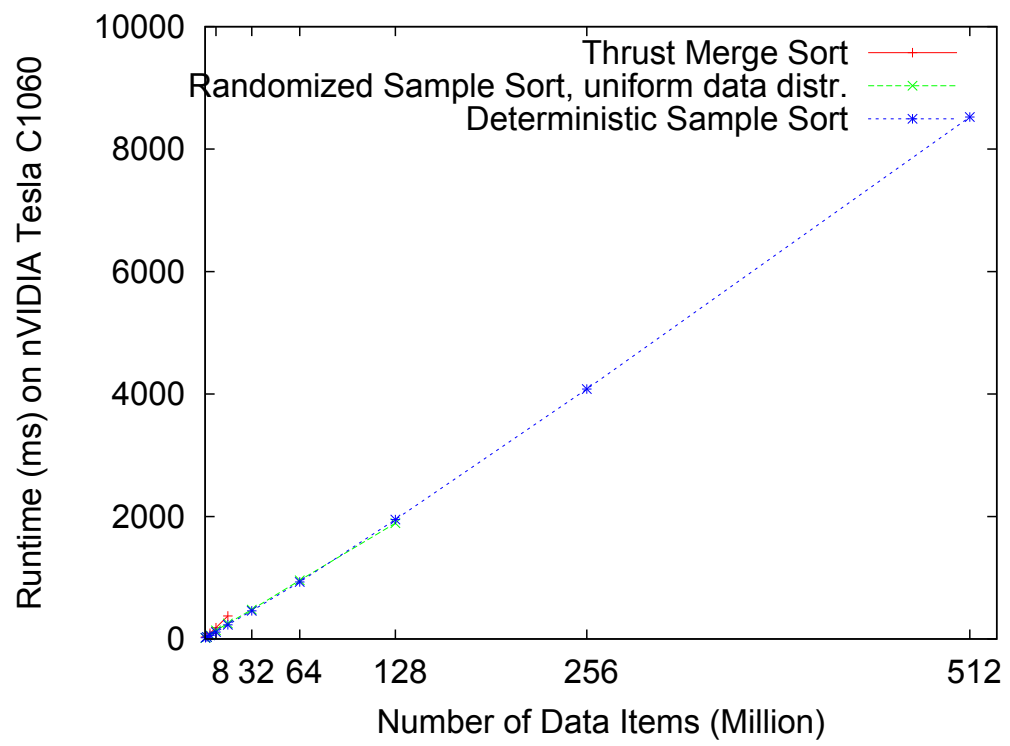

(b) Number of Data Items Up To 512,000,000.

Figure 4.6: Comparison between Deterministic Sample Sort (GPU Bucket Sort), Randomized Sample Sort (GPU S AMPLE SORT) [81] and ThrUST MERGE [98]. Total runtime for varying number of data items on an Nvidia Tesla C1060.([98] and [81] provided data only for up to $16 \mathrm{M}$ and $128 \mathrm{M}$ data items, respectively.) 


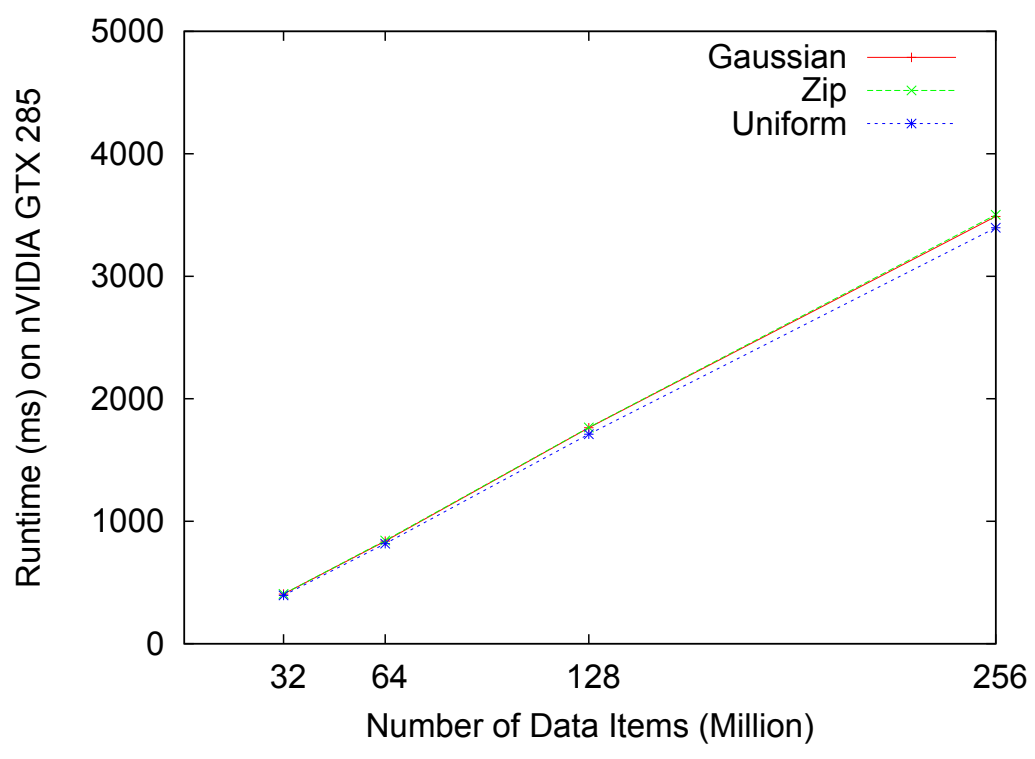

Figure 4.7: Performance of Deterministic Sample Sort (GPU Bucket Sort) for Different Input Data Distributions.

a very close to linear growth rate in the runtime of GPU Bucket Sort for the entire range of data sizes. This is very encouraging for a problem that requires $O(n \log n)$ work. In comparison with randomized GPU SAMPLE SORT, the linear curves in Figures 4.5(b) and 4.6(b) show that our GPU Bucket Sort implementation maintains a fixed sorting rate (number of sorted data items per time unit) for the entire range of data sizes, whereas it is shown in [81] that the sorting rate for randomized GPU SAMPLE SORT fluctuates and often starts to decrease for larger values of $n$.

Based on the experimental results in this section we can observe that the performance of the GPU Bucket Sort has a direct relationship with the number of SM units or simply the number of cores on GPU $(p)$. Furthermore, we observed that by increasing the number of data items to be sorted $(N)$, sorting time increases. The performance also has relationships with other factors such as memory bandwidth. Hence we may provide the following performance model for the GPU Bucket Sort method. Note that we measure the performance of the GPU Bucket Sort according to the sorting time.

$$
\text { SortingTime } \propto \frac{N}{p \times \text { membandwidth }}
$$




\subsection{Summary}

In this chapter, we presented a deterministic sample sort algorithm for many-core Nvidia GPUs, called GPU Bucket Sort. Our experimental evaluation indicates that GPU Bucket Sort is considerably faster than Thrust Merge [98], the best comparison-based sorting algorithm for GPUs, and it is exactly as fast as randomized sample sort for GPUs (GPU Sample Sort) [81] when the input data sets used are uniformly distributed, which is a best case scenario for randomized sample sort. However, as observed in [81], the performance of randomized GPU Sample Sort fluctuates with the input data distribution whereas GPU Bucket Sort does not show such fluctuations. GPU Bucket Sort showed a fixed sorting rate (number of sorted data items per time unit) for the entire range of data sizes tested (up to $\mathrm{n}$ $=512 \mathrm{M}$ data items), whereas it is shown in [81] that the sorting rate for randomized GPU Sample Sort fluctuates and often starts to decrease for larger values of $n$. In addition, our GPU Bucket Sort implementation appears to be more memory efficient since it is able to sort considerably larger data sets within the same memory limits of the GPUs compared to the other GPU sorting implementations. 


\section{Chapter 5}

\section{MCMD-Sort and MCMD-Cube}

\subsection{Introduction}

Most of OLAP servers rely heavily upon a data model known as the data cube [56, 63]. Conceptually, the data cube allows users to view organizational data from different perspectives and at a variety of summarization levels. It consists of the base cuboid, the finest granularity view containing the full complement of $d$ dimensions (or attributes), surrounded by a collection of $2^{d}-1$ sub-cubes/cuboids that represent the aggregation of the base cuboid along one or more dimensions. Typically, the collection of cuboids is represented as a lattice [63] of height $d+1$. Starting with the base cuboid - containing the full complement

of dimensions - , the lattice branches out by connecting every parent node with the set of child nodes/views that can be derived from its dimension list. Figure 5.1 shows the lattice of a 4-dimensional data cube.

In this chapter, we offer a method for parallel building of static data cubes using multicore processors. The next section provides a formal statement for the problem addressed in this chapter.

\subsubsection{Statement of the Problem}

The problem that we address in this chapter is phrased as:

Given a data warehouse with d dimensions and a fact table $F$, what would be a method for parallel building of a static data cube for the data warehouse using multi-core processors. The method is expected to support building of static/batch-based (not real-time) data cubes. It is expected to utilize all processor cores and achieve speedup by increasing the number of cores and parallel disks available in the system. 


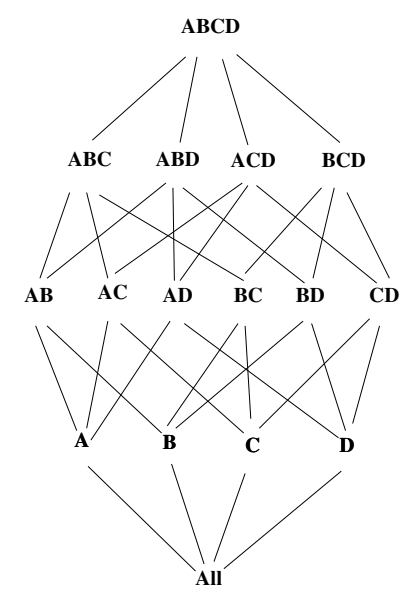

Figure 5.1: The lattice of a data cube with 4 dimensions A,B,C,D. This lattice includes 15 cuboids/sub-cubes/group-bys.

\subsection{MCMD-CUBE Method}

Our parallel data cube construction method called $M C M D-C U B E$ is based on the classical pipesort [14] which decomposes the lattice into a set of chains called pipes, and computes the views in each chain through an external memory sort. Therefore, the performance of our MCMD-CUBE method depends critically on parallel external memory sorting. At the core of our MCMD-CUBE method is a new parallel sorting method termed MCMD-SORT for multi-core processors with parallel disks which outperforms previous methods. Using this parallel external memory sort, we build the lattice of data cube and partition the lattice into pipes. Each pipe is a chain of cuboids from top of the data cube toward its bottom. Cuboids in each pipe share one or more dimensions of the data cube so that after computing a cuboid at depth $l$ of the lattice, it is more efficient and easier to build the next cuboid at depth $l+1$ directly from its parent cuboid in depth $l$. The choice of child(ren) cuboids in each level is a matching problem and can be addressed by the weighted bipartite matching problem[14]. Once all cuboids are assigned to their appropriate pipe in the lattice, it is sufficient to apply our MCMD-SORT algorithm presented in the following to each pipe and then build the pipe from top to the bottom. 


\subsection{MCMD-SORT Method}

In this section we present an outline of our MCMD-SORT algorithm for multi-core processors with multiple parallel disks. Consider a multi-core processor platform with $p$ cores, a local shared memory of size $M$ and a storage of $d$ disks which can be accessed in parallel by the cores. We assume this platform for the remainder of this section. We also assume an input data set of size $N$ data records distributed over the $d$ disks.

For single processor platforms and clusters of single processor machines with distributed storage, multi-way merge has been a common method for data residing in external storage. This method tries to minimize the number of reads and writes to disks since the sorting problem is an I/O-bound problem and needs little computation compared to the slow rate of data transfer from/to disks. In this method, the total data of size $N$ items is partitioned into blocks each of size $M$ that can fit in internal memory. Each of these blocks is loaded from disk into memory and then an internal sorting algorithm sorts each loaded block and writes it back to the disk. After sorting all blocks, a multi-way merge procedure is called and reads data simultaneously from all blocks on disk and merges them into an output buffer. Once the buffer is full, it is written back to disk as the first block including the smallest data records over all $N$ data items. The merge continues until all blocks are completely read and merged. However the merging algorithm will become slow when the total data size $(N)$ and consequently total number of blocks is large relative to memory size $(M)$. In this case, the method tries to perform a recursive division of $N$ into smaller lists and separately apply the same merging method to blocks of each list. The division continues until the number of lists becomes small enough to be merged using the basic merging procedure. If $N$ becomes large relative to $M$, specially larger than a few hundred gigabytes or terabytes, the number of division-merge steps grows with a rate of $O\left(\log _{N} / M^{N}\right)$. This in turn, increases the number of reads and writes to grow logarithmically in $N$. For very large databases, this can become slow and inefficient.

In contrast, our MCMD-SORT uses a scheme similar to deterministic sample sort for clusters [102]. Deterministic sample sort tries to minimize the number of reads/writes to $\operatorname{disk}(\mathrm{s})$ and is able to sort with constant number of reads and writes to $\operatorname{disk}(\mathrm{s})$. This is critical for multi-core processors with parallel disks where compute power is abundant and I/O can easily become the bottleneck. In the remainder of this section we present our 


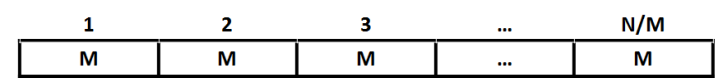

\begin{tabular}{|c|c|c|c|c|c|}
\multicolumn{9}{c}{1} & $\ldots$ & $\mathrm{N}^{1 / 3}$ \\
\hline $\mathrm{M}$ & $\mathrm{M}$ & $\ldots$ & $\mathrm{M}$ & $\ldots$ & $\ldots$ \\
\hline 1 & 2 & $\ldots$ & $\mathrm{N}^{2 / 3}$ & &
\end{tabular}

Figure 5.2: Input data division into smaller blocks of data.

MCMD-SORT method.

Given an input data set of size $N$ data records, a shared memory of size $M$, and $p$ cores, we consider various cases of data size $N$ compared to $M$. If $N$ is smaller than $M^{3 / 2}$, $N$ is small enough to be partitioned into $N^{1 / 3}$ (or less) sublists each of size $M$ or smaller. Otherwise, $N$ data records will be divided into sublists each of size $M^{3 / 2}$. If the number of sublists of size $M^{3 / 2}$ is larger than $N^{1 / 3}$, then a recursive division on $N$ will be performed until we have nested lists and sublists each of size $M^{3 / 2}$ and $M$, respectively, with a total number of $N^{1 / 3}$ (or less) sublists in each list and $N^{1 / 3}$ (or less) lists over all $N$ data records. Figure 5.2 illustrates the division step.

After the division step, the data is partitioned into sublists of size $M$ and possibly one list of size $N^{\prime}$ or smaller. $N^{\prime}$ will be equal to $N$ in case $N \leq M^{3 / 2}$ where only one round of division is needed. According to our calculations, the ratio of the power of $\frac{3}{2}$ is so large that even very large input data sizes (several terabytes) can be sorted with only one round of input data division, which is one advantage of our sorting method over sorting methods that use multi-way merge.

An important feature of our method is that even for extremely large data sets, the division step will never need to recurse at all. For example, with an internal memory $M$ of size 2 gigabytes, which is small compared to today's memory sizes, and one round of data division without recursion, $M C M D$-SORT can sort up to 32 terabytes of 8-byte data records. With $M=8$ gigabytes, available on our test platform, $M C M D$-SORT can sort up to 256 terabytes of 8-byte data records with only one round of division (without any recursion). This is important because one round of division implies a very small, fixed, number of external data accesses.

When the division step is completed, we have blocks of data each of size $M$. Each block is read from disk(s) and loaded into internal memory. In this step, the loaded blocks are sorted and written back to disk(s). Therefore we need to apply an internal memory 
multi-core sorting method to sort each block. Recently, many sorting methods have been proposed for multi-core processors. STL sort available in the standard STL library and libmt sort [82],[62], [97] are state-of-the-art sorting methods for multi-core processors. We tested these sorting methods on in memory data to choose the fastest of them. We observed for our multi-core platform that the latest STL sort implementation with full multi-core support outperforms the other recent sorting implementations for multi-core. Hence we chose STL sort as part of our MCMD-SORT for sorting blocks of size $M$. Note that in case of any new faster internal memory sorting method, STL sort can be easily replaced by that method which in turn makes MCMD-SORT faster as well.

After each block is sorted using STL sort on $p$ cores, $M^{2 / 3}$ samples with equal distance from each other will be taken from $M$ items of the block and will be stored in memory. Then the next block of size $M$ is loaded into memory until all blocks on disk have been sorted.

In the next step, the set of samples stored in memory will be merged. Because the samples from each block are in the ascending order, they together build $N^{1 / 3}$ small sorted buckets. We need to make one sorted list of samples out of them. This can be done using a merging algorithm rather than a complete sorting algorithm. For this step, we apply our multi-core merging algorithm presented in the next section.

After merging all samples, we will have a sorted list of size $N^{1 / 3} \times N^{1 / 3}$ equal to $N^{2 / 3}$ which is equal to $M . M$ samples can be merged in memory without accessing external memory. After merging all samples we choose a subset of samples called global samples (GS). More precisely, we choose $N^{1 / 3}$ equidistant global samples out of the $N^{2 / 3}$ sorted samples and store them in memory. We observe that the number of global samples is so small that we can keep them in memory.

In the next step, we take each global sample $G S_{i}$, and compute bucket $B_{i}$ as follows. We read (for each sorted sublist in external memory) data records that are smaller than $G S_{i}$ (and larger than the the previous $G S_{i-1}$ for later rounds). As shown in [102], the total size of data records in $B_{i}$ will not exceed $2 \times N^{1 / 3} \times \frac{N^{2 / 3}}{N^{1 / 3}}$. Therefore, if we choose $M$ to be half of the available internal memory size, then the total loaded data will not exceed available memory.

After loading data for $B_{i}$, we can merge them instead of using a complete sorting 
method because it consists of $N^{1 / 3}$ sorted sublists. Here, we again apply our multi-core merging algorithm presented in the next section. Finally, each $B_{i}$ is written back to disk. Note that all elements in $B_{i}$ are larger than all elements in $B_{i-1}$. Hence, after completing the process for all $B_{i} \mathrm{~s}$, all of $N$ data records are sorted in external storage.

\subsubsection{MCMD-Merge Method}

Note that all merging steps of MCMD-SORT are executed on data loaded into internal memory. This is an advantage of our MCMD-SORT because it generates sorted sublists that can fully fit into the internal memory of size $O(M)$. We now outline an internal memory multi-core merging algorithm.

Given a total set of $M$ data items in $B$ sorted sublists, and $p$ processor cores, we assign $\frac{B}{p}$ sublists to each core. Each core merges its $\frac{B}{p}$ sublists using a binary merging process. Note that all cores start merging processes simultaneously and they work in parallel on independent data. After this step we will have $p$ sorted sublists. Now we need to merge the $p$ sorted sublists using $p$ cores. Here again we need to divide each of the $p$ sublists into $p$ smaller buckets and then distribute them between the $p$ cores. We need to assign equal work loads to the $p$ cores (i.e. the total number of items assigned to each core should not exceed a maximum threshold). This can be achieved by applying a deterministic sampling method. Note that randomized sampling is also applicable which will result in a slight reduction of work complexity. However, there will not be any guarantee on the total size of buckets assigned to each core. Therefore, we choose to apply a deterministic sampling method as follows.

We start by assigning one sublist to each core. Next, each core takes $p$ equidistant sample items from its sublist. After that, the $p^{2}$ selected samples are sorted using a multicore sorting method. Note that, the total of $p^{2}$ is so small that does not require applying any merging methods. In the next step, core $p_{i}$ takes the $(i p)^{t h}$ sample item out of the $p^{2}$ sorted samples and finds its location in each of $p$ sublists using a parallel binary search algorithm. Next, core $p_{i}$ takes for each sublist $1 \ldots p$, all items smaller than the $(i p)^{\text {th }}$ sample and larger than the $(i-1) p^{\text {th }}$ sample. Therefore, each core $p_{i}$ obtains $p$ buckets containing all data items smaller than the $(i p)^{t h}$ sample and larger than $((i-1) p)^{t h}$ sample. Note that data items in each bucket are already sorted. In the last step, core $p_{i}$ merges its $p$ buckets by 
applying a binary merge method. After all cores completed their merging processes, all of $M$ items are sorted.

\subsection{MCMD-CUBE Algorithm}

Since the appearance of data cube operator in [56], there have been many methods proposed for computation of data cubes. However, computation of data cubes on multi-core processors is still immature. One might consider it as a simple task since, a data cube with $d$ dimensions is composed of $2^{d}$ cuboids which all need to be created. At first sight, there seems to be more than enough parallelism. However, efficient data cube construction methods do not build the $2^{d}$ cuboids independently but use relationships between cuboids to improve efficiency. Examples include the top-down and bottom-up methods discussed in Chapter 2. Any parallel data cube construction method needs to utilize these relationships between cuboids or risk adding additional work. That makes parallelism at the cuboid level complicated. Another problem for parallelism at the cuboid level is that different cuboids can have very different sizes. Therefore, assigning different cuboid computations to different processors can lead to serious load balancing problems.

To overcome this problem, we choose to create parallelism at a finer granularity. We utilize the classical pipesort sequential method [14] and parallelize each of the pipes generated by pipesort. A critical part of generating each pipe as outlined in [14] is external memory sorting. That is why our MCMD-SORT method presented earlier will be a critical component of our solution for building data cube. In the following, we present our $M C M D-C U B E$ Algorithm.

Our method is based on the classical pipesort sequential top-down computation of data cubes [14]. Given a data cube with $d$ dimensions of cardinalities $\left\langle D_{1}, D_{2}, D_{3}, \ldots, D_{d}\right\rangle$ there are $2^{d}$ cuboids to be computed. These $2^{d}$ cuboids can be ordered to be computed based on the lattice of the data cube. An example of this lattice for a data cube with 5 dimensions is shown in Figure 5.3. In this figure, arrows show the order in which pipes and cuboids are computed. In the first step, those cuboids that can be computed from other cuboids are specified. This will form a parent-child relationship between each two levels $i$ and $i+1$ of the lattice. Each parent can have many children and each child can be computed from different parents. Therefore, for each child we need to specify from 


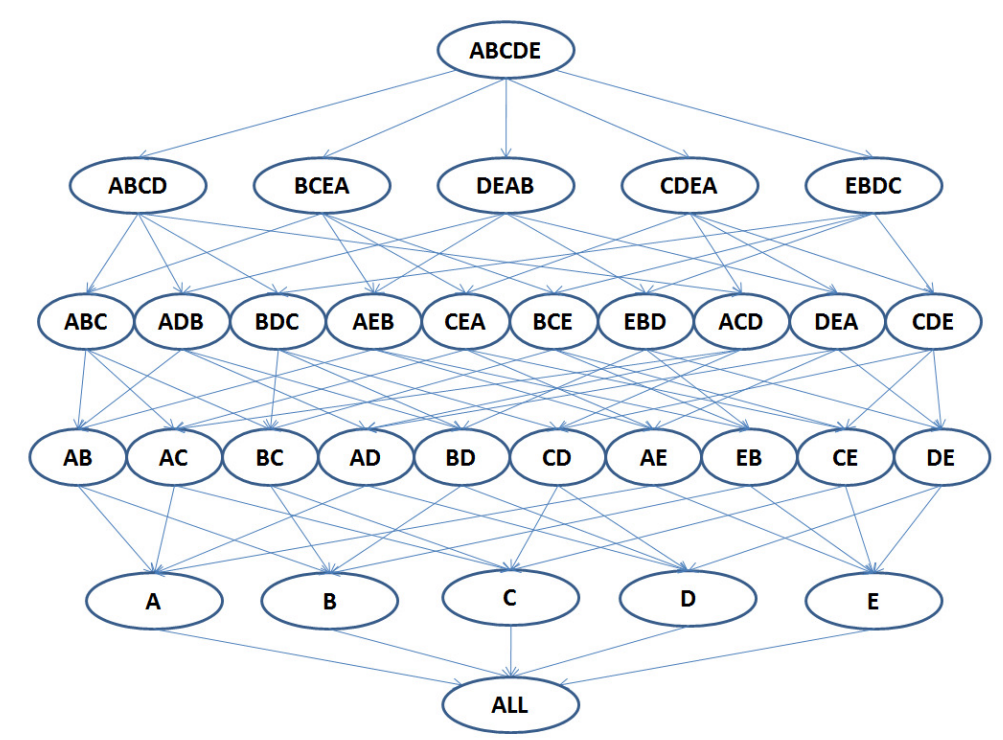

Figure 5.3: Lattice of a 5-dimensional data cube.

which parent cuboid it should be computed. We need to find the parent that applies the minimum cost of computation for the child node. This cost can be calculated according to the estimated sizes of each cuboid which itself can be calculated using cardinalities of the dimensions in a cuboid. The problem of matching parents and children for each two levels $i$ and $i+1$ can be reduced to a bipartite matching problem. Details are presented in [14].

After assignment of each child to a parent, the lattice of the data cube can be divided into pipes each containing a series of cuboids which can be computed from each other. More precisely, we start building each pipe by computing the first parent cuboid which is the largest cuboid in the pipe and then computing child cuboids using the parent. We continue computing the cuboids in the pipe in parent-child order until all cuboids are computed. When we compute a cuboid, we apply our MCMD-SORT method to compute the cuboid using all cores. This guarantees full utilization of all cores while load balancing the computation.

Given a 5-dimensional fact table with dimensions $A, B, C, D, E$, the corresponding lattice shown in Figure 5.3 is partitioned into pipes. For example in Figure 5.3, one such pipe is $A B C D E-A B C D-A B C-A B-A$. The full set of pipes of the lattice in Figure 5.3 is listed in Table 5.1. As shown in the table, we obtain one pipe of length 5 containing 6 


\begin{tabular}{|c|}
\hline ABCDE-ABCD-ABC-AB-A \\
\hline BCEA-BCE-BC-B \\
\hline CDEA-CDE-CD-C \\
\hline DEAB-DEA-DE-D \\
\hline EBDC-EBD-EB-E \\
\hline ADB-AD \\
\hline BDC-BD \\
\hline AEB-AE \\
\hline CEA-CE \\
\hline ACD-AC \\
\hline
\end{tabular}

Table 5.1: 10 pipes of the lattice of Figure reflattice 5-dimensional

cuboids, 4 pipes of length 3 each containing 4 cuboids, and 5 pipes of length 1 each containing 2 cuboids. Note that the cuboid "All" does not need to be computed as it is the fact table of the data cube.

For our parallel MCMD-CUBE method, the computation of pipe is parallelized as follows. We apply MCMD-SORT to the first cuboid of the pipe. Then we parallel aggregate with respect to the dimensions not included in the first cuboid. We note that aggregation can be parallelized by partitioning the sorted cuboid into $p$ segments, aggregating independently and in parallel on each segment, and then aggregating in parallel across the $p$ segment boundaries. Next, we extract all the remaining cuboids in the pipe by aggregating again in parallel with respect to the other dimensions that are removed for those cuboids. Here, we follow again the classical pipesort methods [14] but replace each sequential aggregation by a parallel aggregation as outlined above. The major benefit of this approach is that we still utilize the relationships between cuboids. This leads to work reduction while obtaining maximum parallelism and work balance.

\subsection{Experimental Results}

We now present the experimental results for the MCMD-SORT and MCMD-CUBE methods. We tested our MCMD-CUBE method by building data cubes on both synthetic data and the standard TPC-DS benchmark [109]. We also report the experimental results for our MCMD-SORT method and compare it with the STXXL sorting method [9]. 


\subsubsection{Setup}

Our experimental setup includes a machine with a Sandy Bridge Intel processor and 16 GB of internal memory shared between 4 cores. (Note that, not all of the $16 \mathrm{~GB}$ can be used due to OS limits.) Our external storage consists of 4 parallel disks that can be accessed independently by each core. All implementations were performed in OpenMP with Linux kernel 2.6.38.

Our experiments are divided into two groups: sorting and data cube computation. In the first group of experiments, we tested our MCMD-SORT method on the above platform with a variety of parameters and configurations. In the second group of experiments, we computed data cubes with two sets of data (synthetic and TPC-DS benchmark), and observed close to linear speedup with respect to the number of parallel disks and processor cores.

\subsubsection{Results}

\subsubsection{Results for MCMD-SORT method}

We tested our MCMD-SORT method to evaluate the impact of total data size, number of processor cores and disks, internal memory size, and record size. As a standard configuration, our data includes 8 -byte records and we use all of 8 bytes as sort key. All sorting keys are generated randomly unless otherwise mentioned. Tests of our MCMD-SORT and STXXL sort are performed on the same machine.

The first set of tests are performed to evaluate the impact of growing total data size. In this test, the only variable parameter is total size of the data to be sorted. Data can reside on 1,2 , or 4 disks. In case there are more than one disk, we evenly distribute the data between the disks. Fixed parameters are record size ( 8 bytes) and memory size ( 8 gigabytes). This test includes three parts each performed on a fixed number of disk(s) and processor core(s). The results of this set of tests are shown in Figure 5.4(a-c).

In Figure 5.4(a-c), both the data size and time axis are logarithmic. As can be observed, the difference in performance between our MCMD-SORT and STXXL sort are substantial. The runtime difference between the two methods on 128 gigabytes of data on 1-disk 1core, 2-disk 2-core, and 4-disk 4-core configurations are 4635, 2367, and 1785 seconds, 


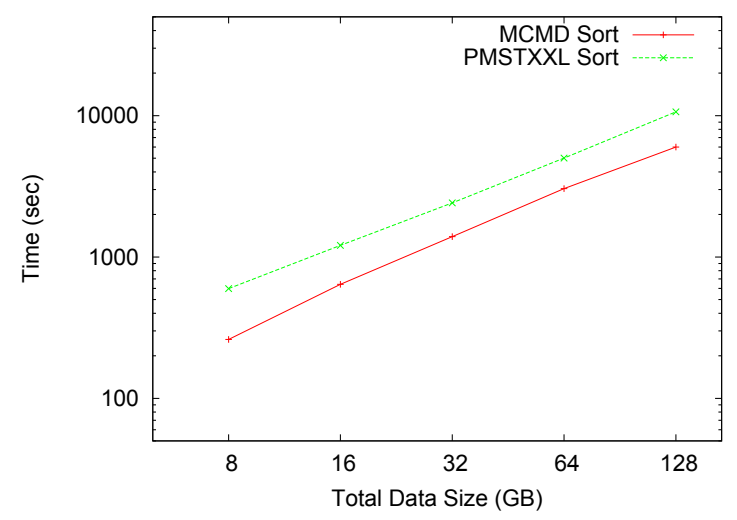

(a)

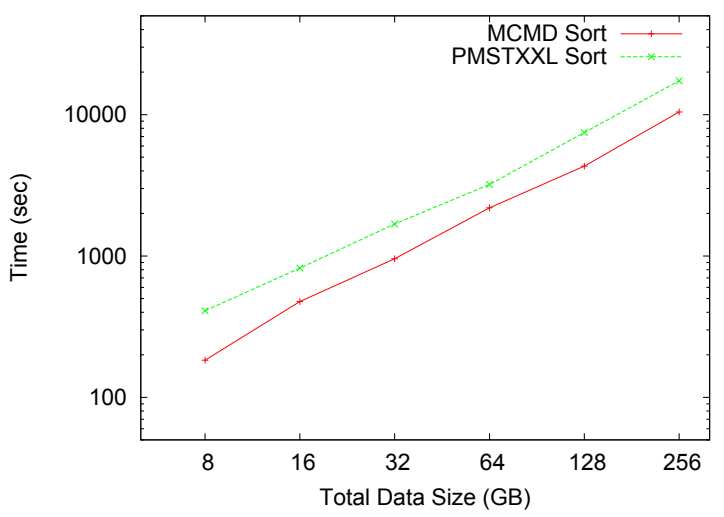

(b)

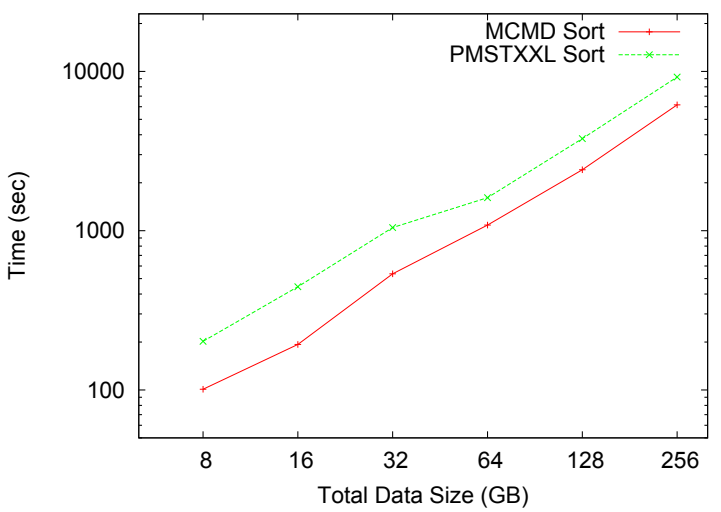

(c)

Figure 5.4: Impact of growing data size on sorting time for different hardware configurations. Memory size: 8 GBytes, Record size: 8 bytes. (a) 1 disk, 1 processor core. (b) 2 disks, 2 processor cores. (c) 4 disks, 4 processor cores. 


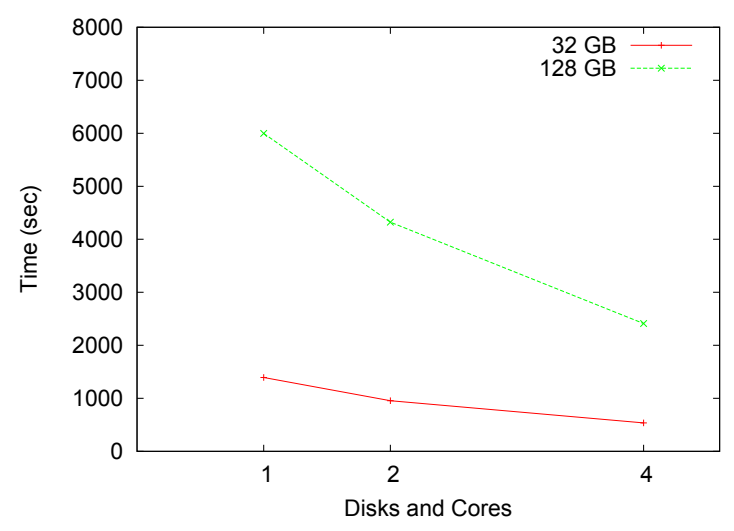

Figure 5.5: Speedup curves for two data sizes, 32 GB and 128 GB. Record size: 8 bytes, Memory size: $8 \mathrm{~GB}$.

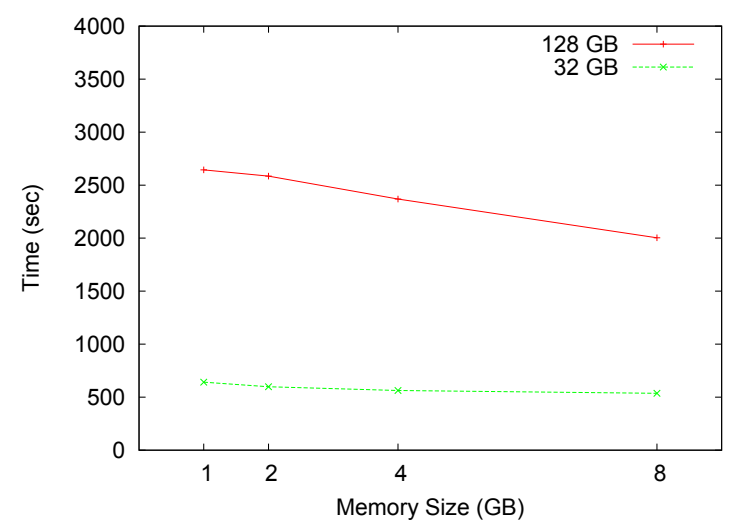

Figure 5.6: Impact of memory size on sorting time. Record size: 8 bytes, 4 disks, 4 cores.

respectively. These differences imply 44\%, 32\%, 48\% of lower sorting time for our our MCMD-SORT compared to STXXL sort.

The second set of tests examines the speedup of our MCMD-SORT method. Figure 5.5 shows speedup curves for two data sizes, $32 \mathrm{~GB}$ and $128 \mathrm{~GB}$, with full memory usage. The only variable parameter in this test is the platform configuration, which can vary between 1-disk 1-core, 2-disk 2-core, and 4-disk 4-core setup. Figure 5.5 shows that our MCMDSORT method sorts faster than STXXL sort by increasing the number of processor cores and disks.

We observed a 30\% improvement on total sorting time when moving from 1-disk 1core to 2-disk 2-core configuration. From 2-disk 2-core configuration to 4-disk 4-core, we observed a $40 \%$ improvement in total sorting time. 
The third set of tests evaluates the impact of memory size. In this experiment, the only variable parameter is the total memory size available to $M C M D-S O R T$ method. Fixed parameters are total data size (32 gigabytes or 128 gigabytes as in the second test) and record size of 8 bytes, using a 4-disk 4-core configuration. The results are shown in Figure 5.6. By increasing the memory size available to the cores, they can load larger blocks of data into memory and sort them. This does have any major impact on the block sorting part of the algorithm. However, in the merging part, we observed a large difference due to the reduced number of blocks to be merged, particularly on large data sets. By increasing memory size, sorting time decreases as the number of merging rounds decreases. We observed that on the 32 gigabyte data set, because total data size is not large compared to total memory size (between 4 and 32 times larger), the decrement in total sorting time is slight. However when dealing with larger data sets such as 128 gigabytes, increasing memory size has a significant impact on the merging part and consequently total sorting time. As shown in Figure 5.6, for a 128 gigabyte data set with 8 GB of memory, total sorting time is significantly lower than with $1 \mathrm{~GB}$ of memory.

The last set of tests for MCMD-SORT method evaluates the impact of record size. In this experiment, we changed the structure of each data record and increased the size of data records from 8 bytes up to 64 bytes. Size of data records is the only variable parameter in this test. The sort key is always 8 bytes but the data field changes from 0 to 56 bytes. By increasing the record size and keeping the same total size for the entire data to be sorted, the number of records decreases. The amount of the data to be moved between memory and disks is fixed (128 GB). For example, when our record sizes are 8 bytes, we have 16 giga records equal to $16 \times 2^{30}$ records. When we increase the record size to 64 bytes, we have 8-byte keys with 56 bytes for data part in each record. In this case, the total number of records to be sorted is 2 giga records equal to $2 \times 2^{30}$ records. As expected, we observe that the total sorting time decreases with increasing the record size because we sort a smaller number of records. However we are transferring the same amount of data, and this prevents us from achieving full speedup. In Figure 5.7, total sorting time decreases with a smaller slope compared to the slopes of the curves in Figure 5.5. This experiment shows that our MCMD-SORT method is I/O bound which is expected for sorting methods. It also highlights the importance of the small fixed number of disk accesses in MCMD-SORT 


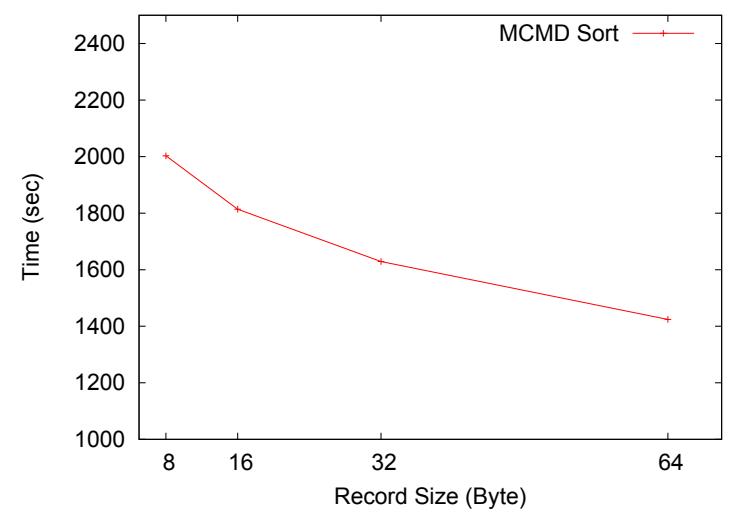

Figure 5.7: Impact of record size on sorting time. Total data size: 128 GB. Memory size: 8 GB. 4 disks. 4 cores.

achieved by the deterministic sampling method.

\subsubsection{Results for MCMD-CUBE method}

We tested MCMD-CUBE on two data sets: a synthetic random generated database and the standard TPC-DS benchmark. Both data cubes are computed on the same hardware platform. The TPC benchmark includes a variety of different data warehouses. In our experiments, we used the TPC-DS data warehouse which is specialized for decision support systems.

Random data sets: In this set of experiments, we created a data warehouse with 5 dimensions. Data records in dimension tables are generated randomly including primary keys that are used in the fact table. We integrated these primary keys in the fact table and generated the fact table. Each record in the fact table contains primary keys of the 5 dimensions. All keys are generated randomly. We refer to these dimensions as $A, B, C, D, E$. Cardinalities of the dimensions are 1024, 512, 512, 1024, and 256, respectively.

We first build the lattice of the data cube and create all pipes in the lattice. Table 5.1 summarizes the chosen pipes of our synthetic database. Next we start reading the fact table and keys in each row and apply our $M C M D-C U B E$ method.

In our sample data cube, we start with cuboid $A B C D E$ and compute it from the fact table by sorting data records based on the order of concatenated keys in cuboid $A B C D E$. Next cuboids in $A B C D E$ pipe can be generated from $A B C D E$ by a single pass over it. We 


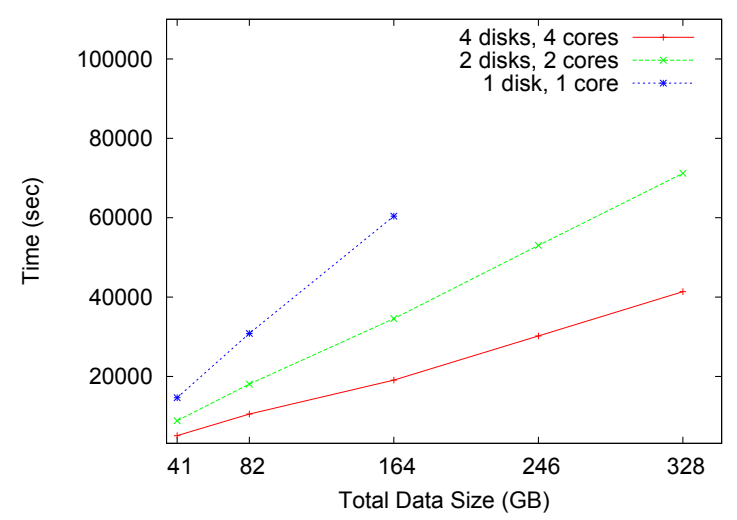

Figure 5.8: Computing data cube of randomly generated data warehouse with 5 dimensions. Memory size: 8 GB. Record size: 164 bytes.

continue computing cuboids of Table 5.1 in the same way until all pipes are built. By building all pipes, data cube is computed.

Although we have 5 dimensions in our database, we added some data fields to the records of the fact table to make it similar to the TPC-DS database. Our fact table records have 164 byte of length which is equal to the average record size in TPC-DS benchmark. When building the data cube, we transfer the whole record to/from internal memory including keys of the 5 dimensions and data part which can contain measures and other attributes of dimensions.

Considering the above approach, our first experiment is to compute a data cube using different hardware configurations with growing the size of fact table. Figure 5.8 summarizes this experiment on our 5-dimensional database.

The figure shows the impact of total data size on the runtime of $M C M D-C U B E$ as well as the speedup obtained through the use of multiple processor cores and multiple disks. The size of the fact table varies from $0.25 \times 164$ GB to $2 \times 164$ GB. With increasing data size, total time of data cube computation increases smoothly. Figure 5.8 also shows the impact of different disk and processor core configurations. The configuration with 4 disks and 4 cores computes the data cube nearly twice as fast as the configuration with 2 disks and 2 cores, indicating close to linear speedup.

The impact of different data distributions on the speedup is shown in Figure 5.9. As we stated earlier, our records and keys were first generated with a uniform distribution. We also generatedd our random data base with Gaussian and Zipfian distributions and then 


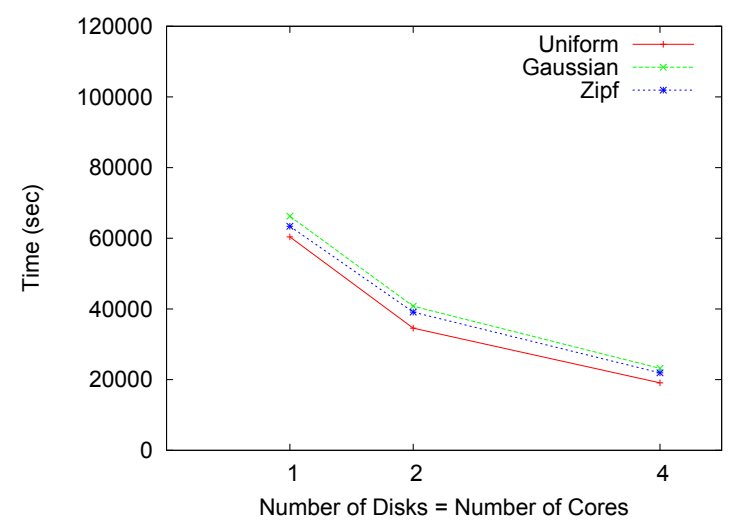

Figure 5.9: Computing data cube on different data distributions. Memory size: 8 GB. Record size: 164 bytes.

used $M C M D-C U B E$ method to build data cubes on these distributions. As expected, there was only minimal (within measurement noise) fluctuation of $M C M D-C U B E$ 's performance in the presence of different distributions. This is due to the fact that MCMD-CUBE uses deterministic sample sort which is not influenced by different data distributions. Figure 5.9 shows that for all three distributions, $M C M D-C U B E$ achieves close to linear speedup. Note that when doubling the number of disks and cores, total computation time will not decrease to a half. This is due to bottlenecks that appear when dealing with reading from/writing to parallel disks. Both hardware and OS cause a bottleneck and prevent us to fully exploit the parallel disks. However, total data cube computation time with 4-disk 4-core configuration is almost $33 \%$ of the total time with 1-disk 1-core configuration.

TPC-DS benchmark: The TPC-DS benchmark is a well-known database benchmark for OLAP and data warehousing. We chose this benchmark to compute data cubes using MCMD-CUBE method. TPC-DS benchmark has 10 dimensions and 7 fact tables in total. The largest fact table is the table Store_Sales. We chose this fact table and computed its data cube using MCMD-CUBE method. Each row of the table contains attributes values of the 10 dimensions and a measure. Average record size of the fact table is 164 bytes. Out of the 10 dimensions, we chose 5 key dimensions and computed a data cube for these 5 dimensions. These dimensions are Item, Customer, Store, Promotion, and Ticket Number.

Figure 5.10 shows the impact of total data size on the runtime of MCMD-CUBE as well as the speedup obtained through the use of multiple processor cores and multiple disks. (Note that the TPC-DS benchmark is only available in specific sizes .) With increasing data 


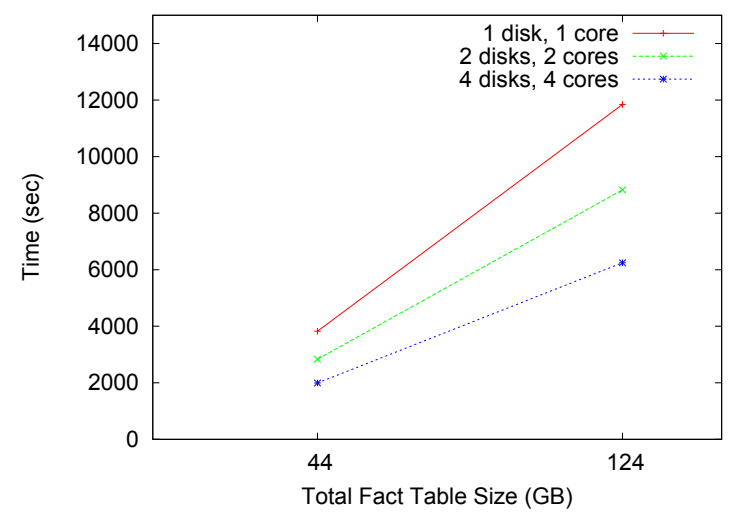

Figure 5.10: Computing data cube of TPC-DS benchmark. Memory size: 8 GB. Record size: 164 bytes.

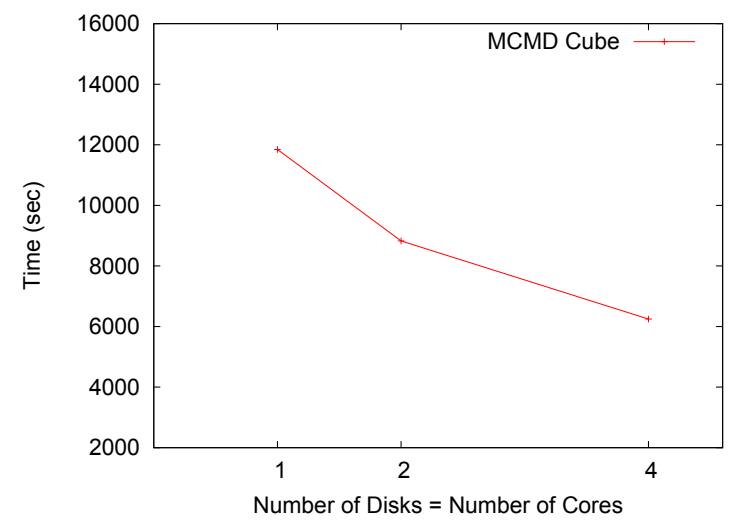

Figure 5.11: Speedup curve for computing data cube of TPC-DS benchmark. Fact table size: 124 GB. Memory size: 8 GB. Record size: 164 bytes.

size, the total time of data cube computation increases smoothly. Figure 5.10 also shows the impact of different disk and processor core configurations. Shown in Figure 5.11, with 4-disk 4-core configuration, $M C M D-C U B E$ computes the data cube nearly twice as fast as the 1-disk 1-core configuration indicating approximately $50 \%$ of optimal linear speedup.

Figure 5.12 shows the impact of internal memory size on the performance of $M C M D$ $C U B E$. Using larger memory sizes allow the method to load more data records each time and create larger pipes with only one pass of read/write over disk(s). This effect is a consequence of the lower work load of MCMD-SORT when sorting with larger memory sizes. Therefore, when we increase the memory size available to $M C M D-C U B E$, the total time for building data cubes decreases. 


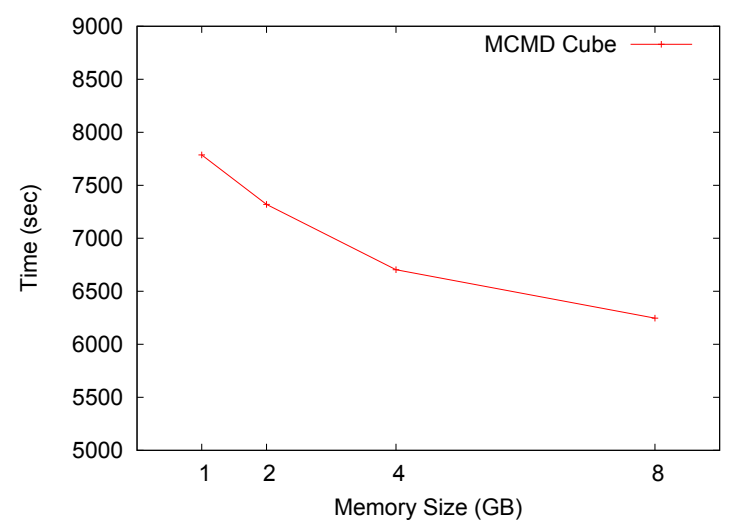

Figure 5.12: Impact of memory size on computing time of TPC-DS data cube. Fact table size: 124 GB. Record size: 164 bytes.

Based on the experimental results in this section for $M C M D-C U B E$, since the performance of the MCMD-CUBE highly depends on the performance of the MCMD-SORT, we observe that there is a direct relationship between the number of cores $(p)$, number of disks $(d)$, memory size $(M)$, and record size $(r)$ with the performance of the MCMD-CUBE. Hence the cube building time has a reverse relationship with the mentioned parameters. On the other hand, the cube building time has a direct relationship with the number of data records in the database $(N)$. Therefore, we may provide the following performance model for the $M C M D-C U B E$ method. Note that we measure the performance of the $M C M D$ $C U B E$ according to the data cube building time.

$$
\text { DataCubeBuildingTime } \propto \frac{N}{p \times d \times M \times r}
$$

\subsection{Summary}

In this chapter, we developed the $M C M D-C U B E$ method, a parallel data cube construction method for multi-core processors with multiple disks. The MCMD-CUBE method applies a parallel external sorting algorithm called MCMD-SORT. The MCMD-SORT is shown to outperform the STXLL sorting method on a Sandy Bridge multi-core platform. We exploited the $M C M D-S O R T$ algorithm in the $M C M D-C U B E$ method to build static data cubes.

Experimental results demonstrated that the $M C M D-C U B E$ achieves close to linear speedup by increasing the number of processor cores and parallel disks. 


\section{Chapter 6}

\section{Real-Time OLAP}

\subsection{Introduction}

This chapter contributes to the main subject of this thesis, what is called Real-time OLAP. We earlier presented the background surveys and discussions in data warehousing, DSS, and OLAP. We refer readers to Chapter 2 for the fundamentals of OLAP and data warehousing. In this chapter, we study Real-time OLAP on two parallel hardware platforms: multi-core platform and cloud platform.

Today, multi-core processors are so common that they can be found in every personal computing device such as desktop computers, laptops, tablets and even cellular phones. In the past few years, these processors have completely replaced single-core processors. As a consequence of this replacement, one needs to make effective use of them by designing proper software frameworks and methods for such processors. The same situation applies to cloud computing. In the recent years, cloud computing has become a standard distributed platform for the tasks that require massive processing and storage capacities. Data warehousing, OLAP, and in particular Real-time OLAP are motivating candidates to utilize multi-core and cloud platforms.

In this chapter, we present two methods called PDC-tree and PDCR-tree and a framework called CR-OLAP for building Real-time OLAP servers on multi-core processors and cloud platform. The PDC-tree and PDCR-tree integrate OLTP and OLAP sides of a data warehouse to supply a Real-time OLAP server. We further discuss the two methods and their features in the following sections.

\subsubsection{Real-timeness}

The methods provided in this chapter are real-time in that they are able to answer every OLAP query without a considerable delay noticeable by human. To this end, we provide a 
few related definitions in the following.

\section{Definition 1: Real-time OLAP}

Assuming a data warehouse with multiple dimensions and a fact table, we define Real-time $O L A P$ as the ability for immediate storage and indexing of arriving data, and the ability for fast retrieval, aggregation, and prompt response for OLAP queries using the most recent updates occurred in the data warehouse.

In order to support real-timeness, Real-time OLAP systems store all of the underlying data in the internal memory of the system known as $R A M$ [89]. This policy allows the system to avoid high latency accesses through I/O to/from external storage. However, it limits the size of the database to the size of the RAM. Nevertheless, the requirements of Real-time OLAP servers and other real-time systems impel them to exclusively utilize the RAM and avoid disk accesses.

\section{Definition 2: OLAP Query}

An OLAP query, in contrast to an OTLP query, accesses large portions of the historical data stored in a data warehouse from multiple dimensions and different hierarchical levels in order to report data records or their aggregation values. The portion of the accessed data may vary from a small OLTP sized query up to $100 \%$ of the data warehouse.

In addition, for a definition of real-time systems we refer to [24]: "Real-time programs must guarantee response within strict time constraints, often referred to as deadlines." Real-time responses are usually in the order of milliseconds. We categorize our real-time responses as a soft real-time response where the reliability of a result degrades after its deadline. In fact, we can phrase the main characteristic of a soft real-time response as to not pose a delay noticeable by human. Since OLAP systems usually provide answers to human users, we can limit our definition to a noticeable time constraint by human. Note that for our real-time solutions, we do not seek hard/firm real-time deadlines where missing a deadline may result in a system failure or wrong results.

In this thesis, we set the noticeable deadline by users in a Real-time OLAP system to 1.0 second. We guarantee that our methods build Real-time OLAP servers that are able to answer every OLAP query no later than 1.0 second after receiving the query in the OLAP server. Considering the above definitions, we call our OLAP methods real-time in that they answer every OLAP query no later than 1.0 second using the latest updates occurred in the 
data warehouse.

\subsubsection{Parallelness}

The offered methods in this chapter are parallel in that concurrent and multiple OLAP operations such as insertions and queries may occur in the OLAP server simultaneously. Multiple threads in a multi-core machine, or multiple machines in a cloud environment, may execute concurrently to provide answers for one or more transactions. This necessitates that offered data structures, framework and algorithms for these data structures support parallelism for both multiple threads and parallel machines. Note that since OLAP is about the analysis of historical data collections, OLAP systems do usually not support data deletion. In this thesis, we do not cover deletion operation in the proposed OLAP methods.

In the following, we present the $P D C$-tree method for multi-core processors in Section 6.2. The PDC-tree method builds a Real-time OLAP server on a multi-core machine. Next in section 6.3, we perform a major and non-trivial upgrade on the $P D C$-tree and present a new data structure called PDCR-tree. The PDCR-tree has several advantages over the $P D C$-tree. We develop parallel algorithms for the PDCR-tree as well as a framework called CR-OLAP to supply distributed PDCR-tree in cloud environments. 


\subsection{Real-Time OLAP on Multi-Core Platforms}

\subsubsection{Statement of the Problem}

We phrase the addressed problem in this section as follows:

Assuming a data warehouse with d dimensions connected to a fact table $F$ in a single machine with a multi-core processor, provide a method to build a real-time OLAP server for the data warehouse. The method should provide real-time responses for the OLAP queries received in the OLAP server. In addition, the method must enable a parallel structure that allows multiple OLAP operations including insertions and queries to occur simultaneously in the OLAP server.

\subsubsection{Introduction}

Our system is an in-memory data management system for OLAP [89] that allows for multiple insert and multiple query operations to be executed in parallel and in real-time. It is based on a new parallel data structure termed PDC-tree. The basic mechanism is outlined in Figure 6.1. In order to process an input stream of OLAP insert and OLAP query transactions in real-time, our PDC-tree data structure allows for the concurrent execution of these transactions through multiple threads executed in parallel on a multi-core processor. Here we employ two types of parallelism: executing multiple OLAP transactions in parallel and further speeding up individual OLAP transactions by assigning each of them multiple parallel execution threads.

The main challenge is the possible interference between parallel insert and query transactions, as well as between parallel inserts operating on the same portion of the tree data structure. For example, each OLAP query has to include all data from prior OLAP insert transactions, including those recent insert transactions that are not yet completed and are being executed in parallel with current OLAP query transactions. A straightforward solution would e.g. lock subtrees on which an insert is being performed. This would however lead to significant wait times for other queries and result in a method where the performance does not scale with increasing number of processor cores. Our goal is the design of a minimal locking scheme which allows concurrent insert and query transactions to move freely and which detects and recovers transactions working on invalid or incomplete data. 


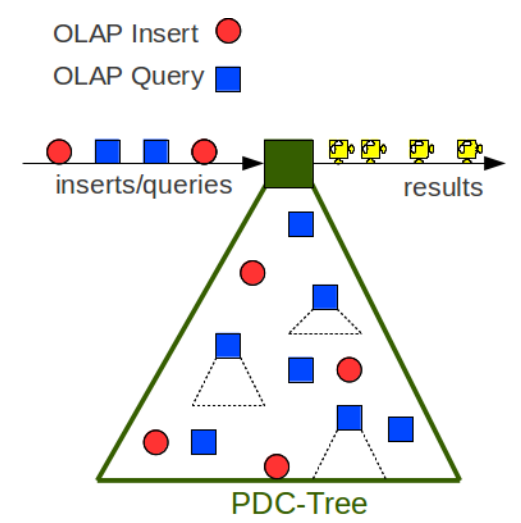

Figure 6.1: A PDC-Tree: In order to process an input stream of OLAP insert and OLAP query transactions in real-time, we provide speedup through concurrent execution of transactions on a multi-core processor.

A parallel Real-time OLAP data warehouse is considerably more complex than concurrent updates and searches that occur in general OLTP databases. One major difference is that OLAP queries may need to aggregate large portions of the database whereas OLTP transaction are more local in nature. Another important difference are the elaborate dimension hierarchies which are typical for OLAP systems. To our knowledge, the only published fully dynamic data structure for OLAP queries on data cubes is the DC-tree introduced by Kriegel et.al. [47], which is a sequential tree-based index structure specifically designed for data warehouses with dimension hierarchies. The DC-tree [47] extends the X-tree [25] and R-tree [60] data structures for multi-dimensional data indexing. We use DC-tree as a base tree for building the $P D C$-tree. In fact, the $P D C$-tree is a parallelization of the DC-tree method.

Note that the parallelization of the DC-tree is not trivial since the DC-tree data structure and its algorithms are not safe against parallel transactions. We need to provide a parallel tree data structure capable of handling millions of data records while providing parallel algorithms for parallel OLAP operations. Such a parallelization requires concurrency mechanisms to assure that the data is stored and reported properly by the concurrent threads. At the same time, the $P D C$-tree is required to provide a real-time answer to every OLAP query. This necessitated applying the mechanisms of parallel R-tree methods into the sequential DC-tree. Examples of published parallel R-tree methods include [21], [107], and [75]. 
We first present a review on the DC-tree method by Kriegel et.al. [47] in the next section, and then provide our parallel DC-tree so called the PDC-tree.

\subsubsection{DC-tree}

Even though the DC-tree was first published more than 10 years ago, and despite the fact that it does provide an elegant algorithmic solution for Real-time OLAP systems, the DCtree data structure has not found its way into commercial OLAP systems. A major problem is performance. For large data warehouses, pre-computed cuboids still outperform realtime data structures but of course with the major disadvantage of not allowing real-time updates, as discussed earlier.

An OLAP database consists of several functional attributes, grouped into dimensions, and some dependent attributes, called measures. For dimensions with more than one functional attribute, these attributes are organized into hierarchy schemas. For example, the dimension customer can have functional attributes region, nation, customer ID. A DC-tree builds a partial ordering and concept hierarchy for each dimension. A concept hierarchy is a tree structure storing for a given dimension all values that occur in the DC-tree at a given time. Using the partial ordering defined by the concept hierarchy for each dimension, the DC-tree extends the X-tree [25] and R-tree [60] by replacing the standard minimum bounding rectangles (MBR) assigned to directory nodes by minimum describing sequences (MDS). An MDS contains for each dimension a set of values at different levels of the dimension hierarchy, and describes a set of hyper-rectangles which together contain the data stored in the respective subtree. The rationale for these minimum bounding sequences is that they enable more efficient queries for the high dimensional data and multiple levels of granularity that are typical for OLAP. The DC-tree includes two operations: Insert (Section 4.1 in [47]) and Range Query (Section 4.4 in [47]). When an insert causes a DC-tree node to exceed its capacity, this is handled by operations Split and Hierarchy Split (Section 4.2 and 4.3 in [47], respectively). For a complete description of DC-tree algorithms, we refer readers to [47]. In the next section, we outline our PDC-tree method. 


\subsubsection{PDC-tree}

The $P D C$-tree is a parallel multi-dimensional index that stores multi-dimensional data in a data warehouse. It is in fact a parallelization of the DC-tree method proposed in [47]. The DC-tree is a sequential structure where only one thread may traverse the tree to perform an update or an OLAP query.

A PDC-tree enables multiple threads to concurrently traverse the tree and perform different OLAP operations requested from clients. A PDC-tree is able to answer OLAP queries including aggregations and OLAP operations such as roll-up, drill-down, slice, and dice over the dimensions of the data warehouse in different hierarchy levels. It is also called real-time in that insertions into the data warehouse may occur while the tree is answering OLAP queries. A PDC-tree answers queries using the latest data in the tree in real-time. Most of the methods proposed for OLAP either lack this advantage or encounter serious challenges to enable it. In the following, we present a detailed discussion on the PDC-tree data structure and its algorithms.

In a data warehouse with $d$ dimensions and hierarchy levels in each dimension, a PDCtree partitions the $d$-dimensional space into $d$-dimensional sub-spaces by defining hierarchies and partial ordering. Assume a data warehouse with the fact table $F$ and a set of $d$ dimensions as $\left\{D_{1}, D_{2}, \ldots, D_{d}\right\}$ where each dimension $D_{i}, 1 \leq i \leq d$ has a hierarchy $H_{i}$ including attributes corresponding to the levels of the hierarchy. The attributes in the hierarchy of dimension $i$ are organized as an ordered set $H_{i}$ of parent-child relationships in the hierarchy levels, $H_{i}=\left\{H_{i 1}, H_{i 2}, \ldots, H_{i l}\right\}$ where a parent logically summarizes and includes its children. Figure 6.17 shows the dimensions and hierarchy levels of each dimension for a 4-dimensional data warehouse.

Assuming the dimension store as $D_{1}$ in a data warehouse, $H_{1}$ is the ordered set of $\{$ Country, State,City $\}$. The DC-tree and PDC-tree are able to partition the $d$-dimensional data space and build sub-spaces that aggregate the data belonging to the different hierarchy levels of each dimension. The sub-space in each dimension may include many hierarchical values for a specific hierarchy level of the dimension. For example, a sub-space in the tree may have the hierarchical values US, Canada for the Store dimension. As another example it may have the values Toronto, Ottawa, Windsor for the Store dimension as three cities in the province of Ontario. A sub-space in this definition matches a subtree in the 


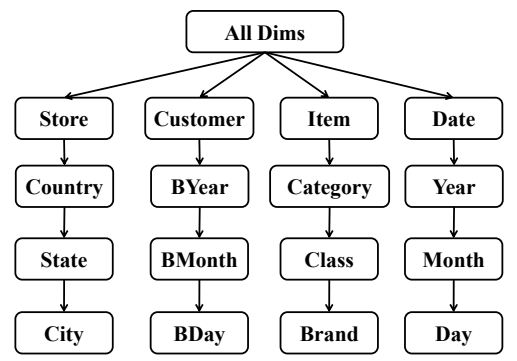

Figure 6.2: A 4-dimensional data warehouse with 3 hierarchy levels for each dimension. The first box for each dimension denotes the name of the dimension.

$P D C$-tree. In fact, each subtree in the PDC-tree covers a sub-space in the $d$-dimensional data warehouse and all of its aggregate values. There is a major difference between the DC-tree and PDC-tree in one side with R-trees in the other side. An R-tree may not index the hierarchical information of the data in the dimensions of a data warehouse. This is an important drawback for R-trees since OLAP users intensively demand an OLAP system to answer queries at different hierarchy and summarization levels. This requires the OLAP system to enable OLAP operations such as roll-up, drill-down, slice, and dice. R-trees are not able to perform these OLAP operations since they do not index hierarchical data.

In order to index hierarchical data in multiple dimensions, the $P D C$-tree forms a regular tree with parent-child nodes that branches out from a root node. At each level of the tree, a parent node is branched out to a maximum of $c$ children defined as the capacity of the tree nodes. Note that the level of tree nodes denotes a different concept from the hierarchy levels in the dimensions. By traversing down in the tree, subtrees contain (and index) smaller $d$-dimensional sub-spaces. However, the smaller sub-space may use the lower hierarchy levels in each dimension. For example, assume a subtree rooted at a node $A$ with a child node $B$ which is the root of a child subtree. The node $A$ for the store dimension may use a set of values in the country level to index all of its child subtrees, while the node $B$ may use the province level for indexing its sub-space. In this case, the province values in the MDS of the node $B$ should be logically contained in a country of the node $A$ 's MDS.

In addition to the above features, a PDC-tree applies other mechanisms to enable concurrent thread traversal in the tree. The mechanisms must not only provide concurrent query processing in the tree, they should also allow real-time insertions to occur in the tree 
while query transactions traverse the tree and return results using the most recent data updated by other threads. Our PDC-tree method consists of two parts: (1) An extension of the DC-tree data structure to help exploit parallel processing and (2) new algorithms PARALLEL_OLAP_INSERT and PARALLEL_OLAP_QUERY to replace the Insert and Range Query operations in [47]. The main challenge for our PDC-tree method is the possible interference between parallel insert and query operations, as well as between parallel insert operations operating on the same portion of the tree data structure. A straightforward solution would e.g. lock subtrees on which an insert is being performed. This would however lead to significant wait times for other queries and result in a method where the performance does not scale with increasing number of processor cores. Our solution consists of three parts:

1. A minimal locking scheme where insert operations only lock the node they are currently updating instead of the entire subtree. This can however result in concurrent other transactions working on invalid or incomplete data.

2. A time stamp mechanism added to the DC-tree data structure which allows for concurrent transactions to detect when they are working on invalid or incomplete data.

3. A set of horizontal sibling links added to the DC-tree structure which allow transactions to recover after they have detected that they were working on invalid or incomplete data.

We first describe our extension of the DC-tree data structure to handle multiple parallel transactions (insert and query operations). There are three types of nodes in a DC-tree: data nodes which are leaves of the tree, directory nodes which are internal index nodes and super nodes which are directory nodes with unlimited capacity. The directory nodes are the backbone of the DC-tree index. Next, we describe our extension of these directory nodes. An illustration of an extended directory node is shown in Figure 6.3. The blue entries show the original DC-tree entries as presented in [47]: $M D S$ is the minimum describing set for the subtree rooted at the given directory node, $M$ is the aggregate measure of all data in the subtree, and a link provides a reference to each child node. The MDS is used for the routing of OLAP queries and the $M$ entry is used for data aggregation in OLAP queries. The red entries in Figure 6.3 are those that we are adding for the parallel DC-Tree. 


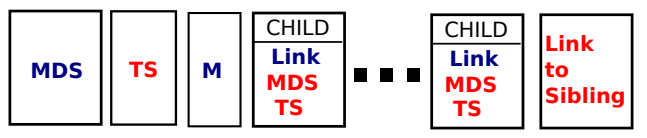

Figure 6.3: Extended structure of a directory node for the PDC-tree. DC-tree entries (blue): $M D S$ (minimum describing set), $M$ (measure), link to each child node. Additional entries for the PDC-tree (red): $T S$ (time stamp), Link to Sibling, $M D S$ and $T S$ for each child node link.

These new entries include: (1) a time stamp $T S$ which records the time of the most recent modification of the directory node, (2) a link to the right sibling of the given directory node, and (3) $M D S$ and $T S$ entries for each child link, recording the $M D S$ and $T S$ values for those children. The $T S$ entry is for concurrent transactions to detect when they are working on invalid or incomplete data. The link to the right sibling will allow transactions to recover after they have detected that they were working on invalid or incomplete data. The $M D S$ and $T S$ entries for each child link will allow to further reduce the number of locks. Finally, we will have the node structure shown in Figure 6.4 for directory nodes in PDC-tree. Next section describes parallel algorithms for OLAP operations.

\begin{tabular}{|c|c|c|c|c|}
\hline MDS & TS & Measures & Link to parent & Link to sibling \\
\hline \multicolumn{2}{|c|}{ Link to Child 1 } & $\ldots$ & \multicolumn{2}{|c|}{ Link to Child c } \\
\hline
\end{tabular}

Figure 6.4: A directory node in PDC-tree and its fields in order from left to right: MDS: Minimum Describing Sequence, TS: Update Time Stamp, M: The set of measures, Links to children, Link to the sibling node rooted at the same parent.

\subsubsection{Algorithms}

In this section, we will outline our method in more detail by presenting the new $P D C$-tree operations PARALLEL_OLAP_INSERT and PARALLEL_OLAP_QUERY, respectively.

\subsubsection{Algorithm PARALLEL_OLAP_INSERT}

An outline of our PARALLEL_OLAP_INSERT method is shown in Algorithm 2. In the following, we will discuss this algorithm in more detail. For a new data record $N$, the 
algorithm starts tracing down the tree using the MDS information (Steps 2-4). At each directory node, three cases may occur. If $N$ is contained in the MDS of exactly one child, then the algorithm proceeds to that child (Step 2). If $N$ is contained in the MDS of more than one child (overlap), then the algorithm proceeds to the child with the smallest subtree (Step 3) in order to balance subtree sizes. If $N$ is not contained in the MDS of any child, then $N$ needs to be added to the child whose MDS update leads to a minimum overlap between children in order to maintain the efficiency of search queries (Step 4). Algorithm 2 performs this operation without any node locking by first creating a copy of the respective directory node, performing all of the above operations on the copy, and finally inserting the new directory node with a single link update. Note, however, that search queries passing through this node during the update may not become aware of the update and may therefore miss the newly inserted data item $N$. As discussed later, Algorithm 3 for search queries will detect and correct this with the help of the time stamp (TS) and right sibling entries added to the modified directory node structure. After Steps 1 to 4 of Algorithm 2 are completed, a leaf directory node has been found where the new data item $N$ can be inserted. The remaining Steps 5 to 11 will trace the path back to the root and update the MDS entries of all directory nodes on the path. Note that, during this process at most two nodes are locked at any point in time (double-locking strategy): the current node and its parent. This is necessary to correctly perform the split operation in Step 8 which is required when a directory node's capacity is exceeded because of the new entry. In this case, a split operation has to be performed where directory node $D$ is split into two directory nodes $D$ and $D^{\prime}$. For the split, we applied the sequential method in [47], Sections 4.2 and 4.3. As discussed at the end of this section, we also developed a parallel split operation. After $D$ is split into two nodes $D$ and $D^{\prime}$, node $D^{\prime}$ becomes the right sibling of $D$ and we need to update the right sibling links accordingly. Furthermore, the time stamps of $D$ and $D^{\prime}$ will be set such that $D^{\prime}$ receives the old time stamp of $D$ (before the split) and $D$ receives a new time stamp representing the current update. As discussed in the following Section 6.2.5.2, this will be important for concurrent OLAP queries.

At any point in time, our $P D C$-tree data structure will be performing multiple concurrent PARALLEL_OLAP_INSERT operations executed by different threads of the multi-core processor. Any two such PARALLEL_OLAP_INSERT operations will not interfere unless 


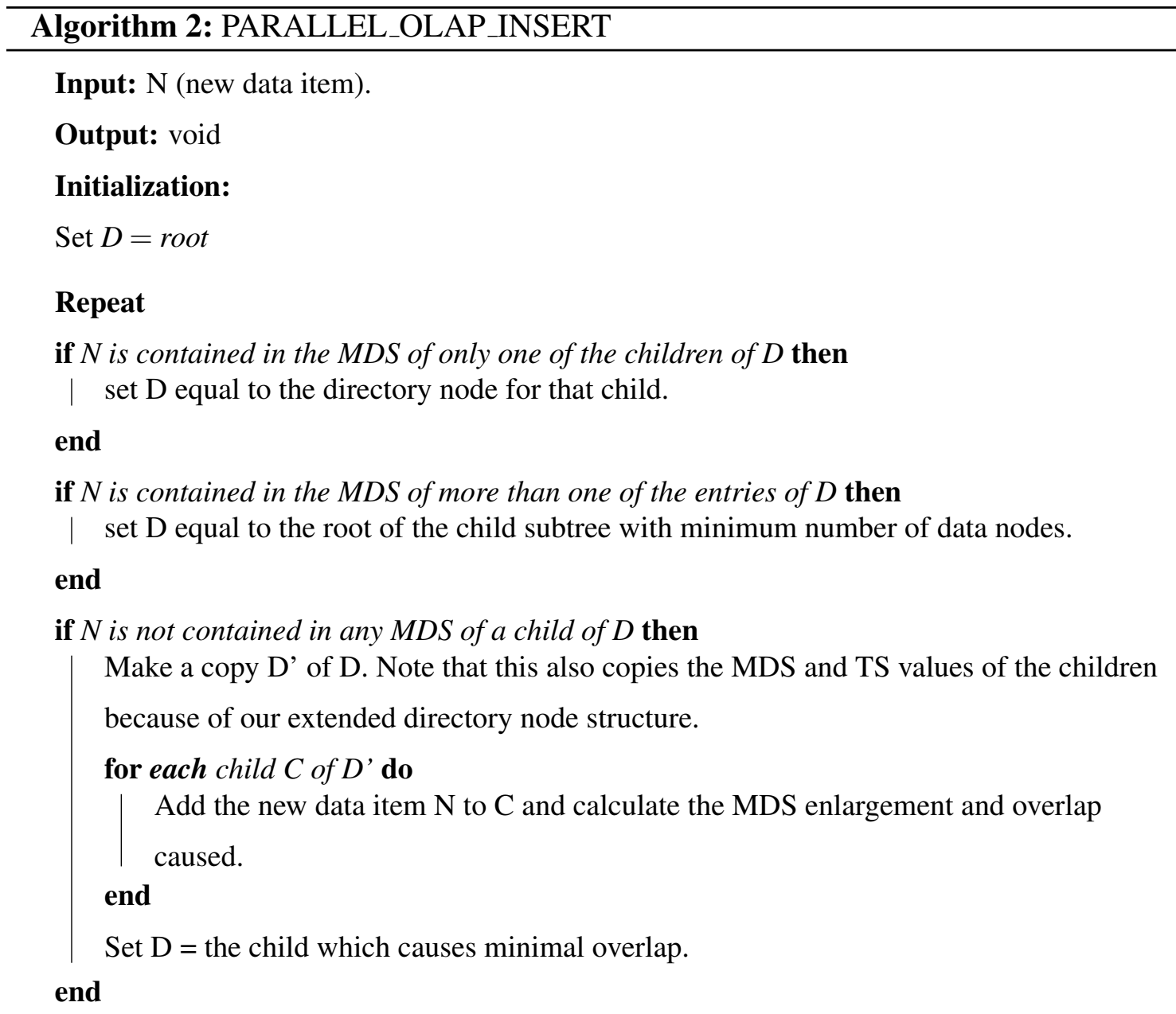

Until: D is a leaf node.

Acquire a LOCK for D.

Insert data item $\mathrm{N}$ into D and update the measure, MDS, and time stamp (TS) of D.

\section{Repeat}

Acquire a LOCK for the parent of D.

if capacity of $D$ is exceeded then

Split D into two directory nodes D and D' as outlined in [47], Sections 4.2 and 4.3.

Make D' the right sibling of D and update the right sibling links accordingly.

Set the time stamp TS of D' equal to the old TS value for D and assign D a new time stamp TS representing the current update.

end

Update the Measure and MDS fields for the parent of D.

Release the LOCK for D.

Set $\mathrm{D}=$ parent of $\mathrm{D}$.

Until no further update required OR $\mathrm{D}=$ root. 
they are attempting to perform a node split on the same directory node at the same time. This is our minimal node locking condition. Otherwise, there are no locks. In particular, at any point in time there can be multiple concurrent node split operations happening in the PDC-tree.

While the PARALLEL_OLAP_QUERY operations presented in the following Section 6.2.5.2 are also individually parallelized by applying multiple threads to each OLAP query operation, each individual PARALLEL_OLAP_INSERT operation as outlined above is single threaded. We experimented with parallelizing the node split operation (Step 8) in Algorithm 2 which is the most time consuming part. As outlined in [47], Sections 4.2 and 4.3, a node split for a directory node $D$ considers all seed pairs of entries in $D$, computes for each pair of seeds a node split by assigning all remaining entries to the closest seed element, and then chooses the best split of $D$ with minimum overlap and volume. Clearly, one can also perform this operation in parallel using multiple threads. We experimented with this and it turns out that parallelizing the split operations is usually not beneficial for current multicore processors. The reason is that a benefit would only occur if there are free compute resources available for additional threads. However, for a typical OLAP workload, such as the TPC-DS benchmark used in our experiments, the number of concurrent transactions on the $P D C$-tree data structure usually exceeds the number of hardware threads available on current multi-core processors. In fact, parallelizing the split operations created additional context switching overheads in our experiments and we therefore switched the node split back to the sequential code. However, for future multi-core processors with many more processor cores, switching on the internal OLAP query parallelization described above could bring further performance benefits.

\subsubsection{Algorithm PARALLEL_OLAP_QUERY}

Our PARALLEL_OLAP_QUERY method shown in Algorithm 3 answers OLAP aggregate queries. For a query range $R$ (hyper-rectangle on a cuboid/aggregate), it reports the aggregate measure value of all data items contained in $R$ (e.g. total value of sales). In addition to the extended directory nodes outlined in Section 6.2.4, we add a stack $S$ to each query in order to ensure proper execution. Stack $S$ controls the tree traversal as well as the error recovery from a detected interference with a parallel insert. The query process starts at 
the root and proceeds downwards. At each directory node, all children are evaluated for possible overlap with the query range $R$ (Step 4.3). For those dimensions where the child MDS and query are at different levels of the dimension hierarchy, the one with lower level needs to be converted to the higher level (Step 4.3.1). If a child MDS fully contains $R$, then the entire subtree is part of the result (Step 4.3.2) and the query does not need to search inside the subtree. If a child MDS overlaps $R$, then that child is pushed into the stack $S$ for further examination (Step 4.3.3). This leads to a branching off into multiple subtrees for those directory nodes where multiple children overlap $R$. The stack mechanism ensures that these subtrees are traversed in depth-first order. For parallel transactions, the problem arising is that while one subtree of a directory node is being searched, the directory node itself could be modified by a parallel insert operation (e.g. a directory node split). This problem is addressed in the IF statement at the beginning of Step 4 together with Steps 4.1 $\&$ 4.2. Assume that the search branches off into a subtree of node $D$ and that, during that time, node $D$ is modified by a parallel insert. When the search returns to node $D$, its "old" version $D^{\prime}$ is on top of stack $S$ and a comparison of the time stamp of $D^{\prime}$ and the current time stamp of $D$ detects a difference, indicating a parallel update. Figure 6.5 shows an illustration. Directory node $D_{1}$ is pushed into stack $S$ and then directory nodes $D_{2}$ and $D_{3}$. In the meantime, a concurrent insert causes a directory node split for $D_{1}$ that updates its time stamp, creates a new sibling node $D_{4}$ with the old time stamp of node $D_{1}$, and re-distributes the children. Node $D_{3}$ is now a child of node $D_{4}$. The concurrent search query completes its task and pops nodes $D_{2}$ and $D_{3}$ from the stack. However, when the search query wants to pop node $D_{1}$ it detects that node $D_{1}$ has been split.

In order for the search query to recover and report the correct result, the list of siblings maintained by the "Link to Sibling" pointers shown in Figure 6.3 should be traversed and added to stack $S$, thereby making sure that the subtrees are re-visited and the newly inserted item is found. Note that, when a directory node is split, its children are re-distributed between the original and the newly added directory node. Therefore, we need to be careful when re-visiting subtrees to not "double count" items by adding them twice to the aggregate measure value $R$. For each item in a re-visited subtree, the time stamps recorded and contents of the stack $S$ are sufficient to determine whether this item has already been counted or not. 
Algorithm 3: PARALLEL_OLAP_QUERY

Input: R (MDS of the given query range), D (the root node).

Output: Result (aggregate measure of all data items contained in R).

\section{Initialization:}

Initialize a stack $S$.

Push D into stack S.

\section{Repeat}

Pop top item D' from stack S.

Set $\mathrm{D}$ to the tree node corresponding to D'.

if the time stamp (TS) of D' is smaller (earlier) than the time stamp (TS) of D then Using the "Link to Sibling" field in directory nodes, traverse the list of siblings of D. Push all siblings with time stamp (TS) larger (later) than the parent of D into stack S.

Push D into stack S.

else

for each each child $C$ of $D$ do

For each dimension of $\mathrm{C}$ where $\mathrm{C}$ and $\mathrm{R}$ are at different level in the dimension hierarchy, convert the lower level entry to the higher level.

if $M D S$ of $C$ is contained in $R$ then

I add $\mathrm{C}$ to the Result set.

end

if $M D S$ of $C$ overlaps $R$ but is not contained in $R$ then

| create a new thread that executes Algorithm 3 with input parameters R and $C$. end

end

end

Until all threads are completed and the stack S is empty. 


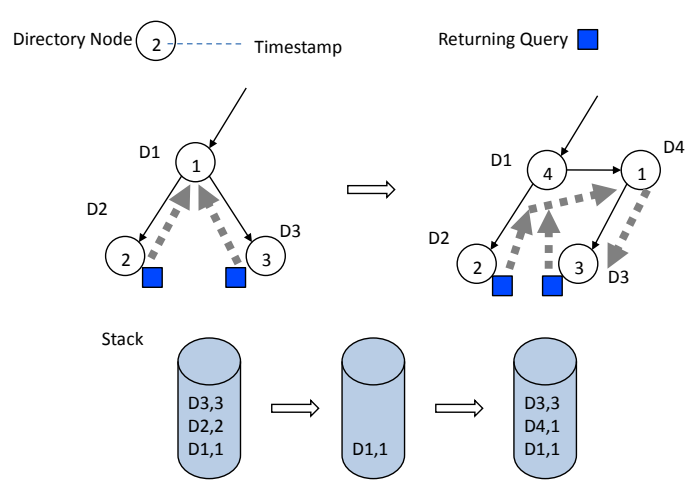

Figure 6.5: Usage of stack and timestamp mechanism to detect updates on already counted nodes in an OLAP query.

As outlined earlier, our system employs two types of parallelism: executing multiple OLAP operations in parallel and further speeding up individual OLAP operations by assigning each of them multiple parallel execution threads. For a directory node $D$, if multiple children of $D$ have an MDS that overlaps the query range $R$, then these multiple subtrees need to be traversed. If this happens close to the root node then the sizes of these subtrees can be substantial. Therefore, we parallelize the search of the subtrees as shown in Step 4.3.3 of Algorithm 3. More precisely, for each child $C$ of $D$ that overlaps $R$ but is not contained in $R$ we create a new thread that executes Algorithm 3 with input parameters $R$ and C.

We note that our PARALLEL_OLAP_QUERY method creates no locks whatsoever and therefore creates no slowdown between parallel transactions. However, it can create additional work during the recovery phase of interfering parallel transactions. This could potentially affect the scalability of our method. To which degree this does actually happen will be determined in our experimental evaluation in the following Section 6.2.6.

\subsubsection{Experimental Results}

In this section, we present our experimental evaluations on the PDC-tree method on multicore machines. We setup a test environment in the next section and then we present the results of our experiments. 


\subsubsection{Setup}

Multi-core Platforms: For our experimental evaluation, we used two multi-core hardware platforms shown in Table 6.1:

- An Intel Sandy Bridge processor with 4 cores (8 hardware threads via hyper-threading) and 16 GB memory.

- A dual socket Intel Westmore EX with 20 cores (40 hardware threads via hyperthreading) and 256 GB memory.

Input Data Set: The main goal of our experimental evaluation is to determine how our parallel tree scales when we increase the number of processor cores. As discussed in Section 6.2.5, our $P D C$-tree algorithms use a minimal locking scheme which allows parallel transactions to execute independently without concern about interference. Should such interference occur, our algorithm detects this and corrects the result during a recovery step. How much overhead is created during those recovery steps depends on the actual data and queries. Clearly, using random data and random queries does not provide a correct estimate because one would expect it to evenly distribute the parallel threads over the PDC-tree which would be a best case scenario for the amount of interference between concurrent transactions. What is needed is a realistic set of data and queries that provides real-life scenarios. We selected the TPC-DS "Decision Support" benchmark by the Transaction Processing Performance Council [109] for our experiments. The TPC-DS benchmark provides transactions that model the decision support system of a retail product supplier. It includes OLAP queries and data insertions. (Decision support systems are based on historic corporate data and usually do not include data deletions.) The TPC benchmark and its variants are the standard and most widely used benchmarks for OLAP. As stated on the TPC website, "although the underlying business model of TPC-DS is a retail product supplier, the database schema, data population, queries, data maintenance model and implementation rules have been designed to be broadly representative of modern decision support systems" [109].

Implementation and Configuration: Our PDC-tree algorithm outlined in Section 6.2.5 was implemented in C++ with OpenMP, and compiled/executed on Linux kernel 2.6.38 using g++ 4.5.2. For each experiment, we first built an initial PDC-tree with TPC-DS 


\begin{tabular}{lll}
\multicolumn{4}{l}{ Table 6.1: The two hardware platforms used in our experiments. } \\
\hline \hline Processor & Number of Cores & Memory Size \\
\hline Intel & 4 Cores & \\
Sandy Bridge & $\begin{array}{l}\text { 8 Hardware Threads } \\
\text { (Hyper-threading) }\end{array}$ & 16 GB Memory \\
& & \\
Intel & 20 Cores \\
Xeon Westmere & 40 Hardware Threads & 256 GB Memory \\
EX (2 Sockets) & (Hyper-threading) & \\
\hline \hline
\end{tabular}

data and then tested our system with a stream of TDC-DS insert and query transactions as shown in Figure 6.1. Another important parameter influencing query time (sequential or parallel) is the amount of data that an OLAP query needs to aggregate. Clearly, a query that computes an aggregate over $5 \%$ of the database has a much smaller workload than a query that computes an aggregate over $50 \%$ of the database. We refer to this parameter as query coverage, and we evaluate in our experiments how our PDC-tree method performs for different query coverages.

\subsubsection{Results}

Experiments on Intel Sandy Bridge: Figures 6.6(a\&b) show the performance of the initial $P D C$-tree building phase. Here we show the average transaction response time and throughput when 400,000 initial PARALLEL_OLAP_INSERT operations (with TPC$D S$ data) are executed on 1, 2, 4, 8, and 16 threads, respectively. Figures 6.6(c\&d) show the average transaction response time and throughput for a subsequent set of 1,000 PARALLEL_OLAP_INSERT operations into an OLAP database with 400,000 loaded items. The performance in Figures $6.6(\mathrm{a} \& \mathrm{~b})$ is lower because the first inserts into an initially small tree data structure create a relatively large number of expensive directory node splits and have a very high probability of query interference. As shown in Figures 6.6(c\&d), for a database with 400,000 loaded items, performance improves significantly. In general, we observed that performance is better for larger data warehouses. The speedup shown in Figures $6.6(\mathrm{c} \& d)$ is approx. $40 \%$ of the maximum theoretically possible linear speedup.

Considering that the cores of the Sandy Bridge processor share resources (e.g. memory 


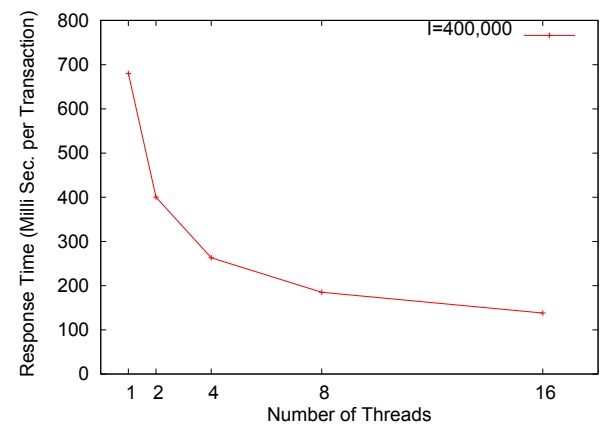

(a)

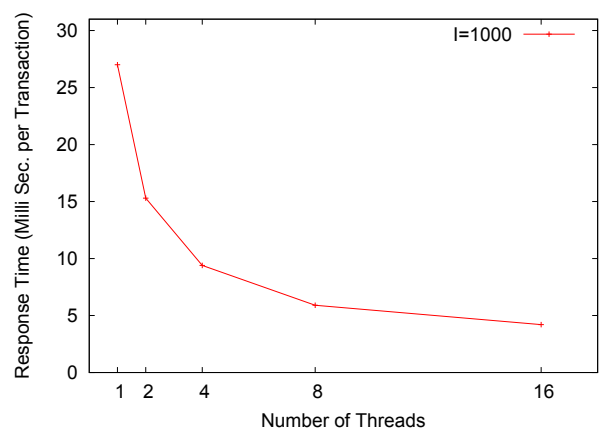

(c)

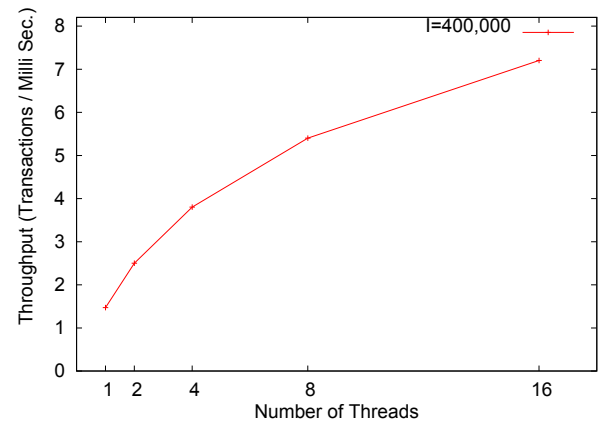

(b)

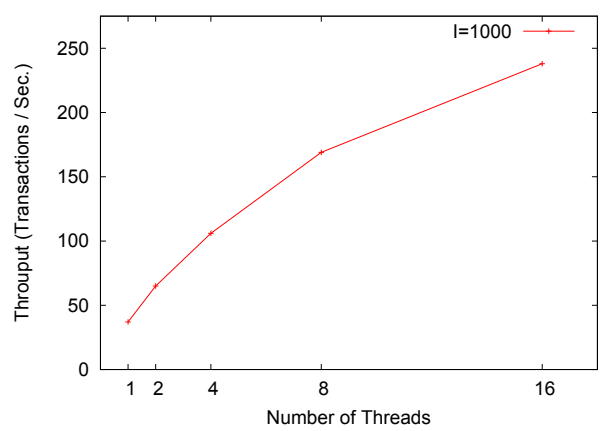

(d)

Figure 6.6: Parallel OLAP insertion performance of the PDC-Tree as a function of the number of parallel threads for Intel Sandy Bridge. (a) \& (b) Average transaction response time \& throughput for 400,000 OLAP insertions to build an initial database. (c) \& (d) Average transaction response time \& throughput for a subsequent 1,000 OLAP insertions into the built database.

bus), this is an encouraging result for a fully running system on "real-life" benchmark data. The Sandy Bridge platform is clearly a too small platform with insufficient memory for a sufficiently large database. For larger data warehouses on a larger platform, as discussed later in this section, the performance results are considerably better. Figures 6.7, 6.8 and 6.9 show the performance (transaction response time and transaction throughput) for a stream of PARALLEL_OLAP_INSERT and PARALLEL_OLAP_QUERY transactions (using TPC-DS benchmark data) executed on 1, 2, 4, 8, and 16 threads, respectively.

Note that the single thread code on one processor core is the sequential code only with all parallelization code (and possibly resulting overhead) removed. The different curves correspond to different ratios between the number of insertions and queries (I OLAP insertions and $Q$ OLAP queries). Since the performance of a (sequential or parallel) OLAP 
query is strongly influenced by the query coverage, we provide three different graphs, Figure 6.7, Figure 6.8 and Figure 6.9 for queries that aggregate $1 \%, 5 \%$ and $25 \%$ of the entire database, respectively. In each case, we show response time and throughput for 1, 2, 4, 8, and 16 threads. In order to better show the speedup achieved, we show each set of curves in linear scale as well as in log-log scale. Our main observation is that we achieve about $40 \%$ of optimal linear speedup. Here, for throughput graphs we define speedup as the ratio of the number of completed transactions by 1 thread over the number of completed transactions by more than a thread in a sec/milli sec. For Response time graphs, speedup is defined as the ratio of the time taken to complete a single transaction by 1 thread over the required time by more than one thread. Figure 6.6 Considering that the cores of the Sandy Bridge processor share resources (e.g. memory bus), this is an encouraging result for a fully running system on such a small platform. We also observe that query transactions are considerably slower than insert transactions. This is typical (also for sequential transactions) since an insert corresponds to just one path down and back up the tree whereas query transactions may need to search multiple subtrees and may need to aggregate a sizeable portion of the database. In the mix of parallel insert and query transactions on the same database, the speedup observed was best for the hardest case of queries only, which is where speedup is most needed in practice.

In addition to the speedup observed, we also compared the running times of our PDCtree implementation with those of a current multi-threaded database system. We chose MySQL [5], a well-known open source database system which has been optimized for multi-core parallelism. On the same Intel Sandy Bridge architecture, we ran our PDC-tree implementation against MySQL with its multi-threading option set to "maximum" parallelism, i.e. its fastest setting. For both, the PDC-tree and MySQL, we first executed the same sequence of 400,000 data insertions from the $T P C-D S$ data set and then, for query coverages $1 \%, 25 \%, 75 \%$ and $100 \%$, the same sequences of queries. Table 6.2 summarizes the results. For $1 \%$ query coverage, multithreaded MySQL performs essentially at the same speed as the PDC-tree. Such queries aggregate only a very small portion of the database and are easy to answer directly in standard database systems like MySQL. However, as we increase query coverage, the queries become much more time consuming for 


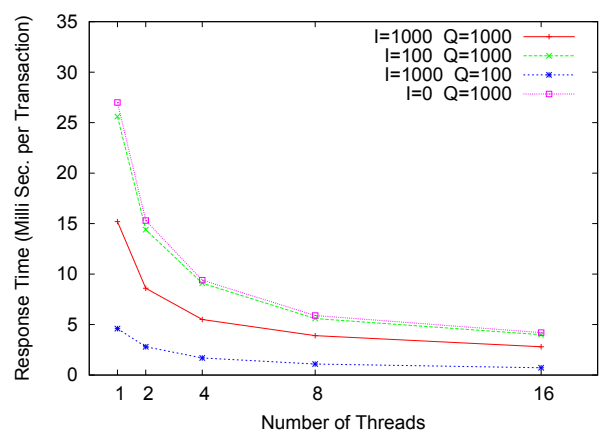

(a)

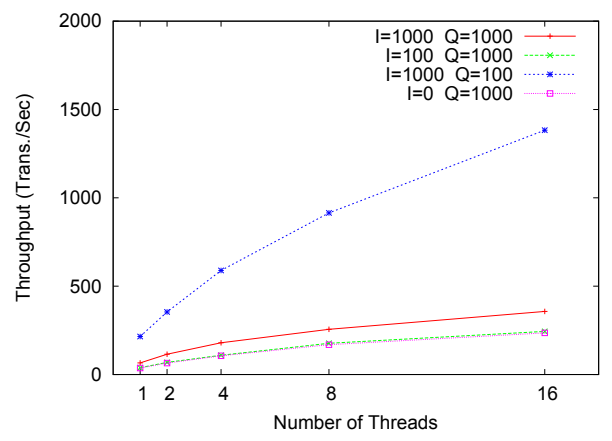

(c)

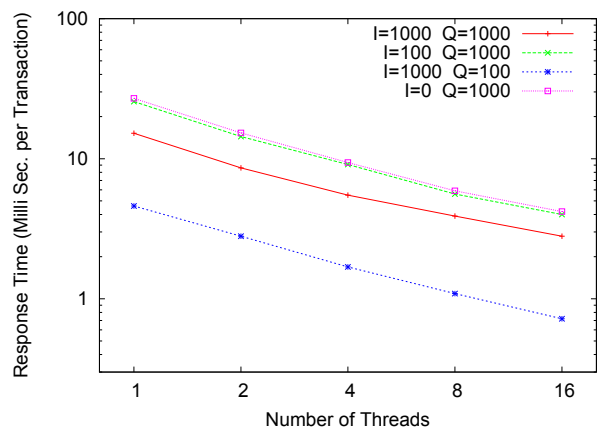

(b)

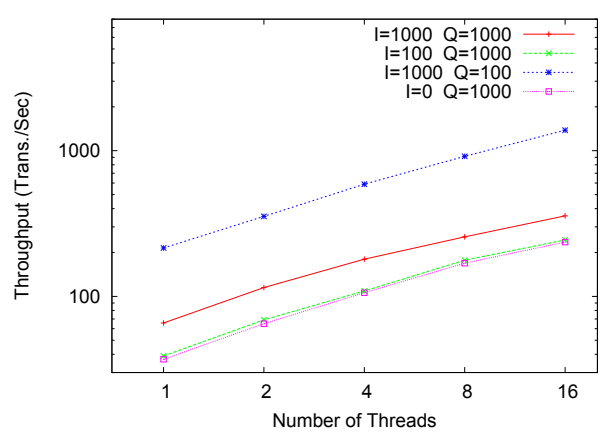

(d)

Figure 6.7: Parallel OLAP transaction performance of the PDC-Tree as a function of the number of parallel threads for Intel Sandy Bridge. Mixed input of $I$ OLAP insertions and $Q$ OLAP queries. Queries aggregate $1 \%$ of database. (a) Average response time, linear scale. (b) Average response time, log-log scale. (c) Throughput, linear scale. (d) Throughput, $\log -\log$ scale.

MySQL and the PDC-tree starts outperforming MySQL by a wide margin. For the extreme case of $100 \%$ query coverage, the $P D C$-tree can pick up the aggregated result at nodes very close to the root and performs extremely fast, two orders of magnitude faster than MySQL. For 25\% and 75\% query coverage, our PDC-tree implementation outperforms multi-threaded MySQL by approximately a factor five.

We also observe that in Table 6.2 , for $25 \%$ and $75 \%$ query coverage, the single thread performance of our PDC-tree implementation approximately matches the performance of multi-threaded MySQL. If we compare that with e.g. Figure 6.9(a), this means that the baseline for the speedup observed for our PDC-tree Figure 6.9(a) (i.e. its single thread performance) is similar to the performance of multi-threaded MySQL. We are highlighting this because speedup is easy to achieve with respect to a slow sequential performance but in 


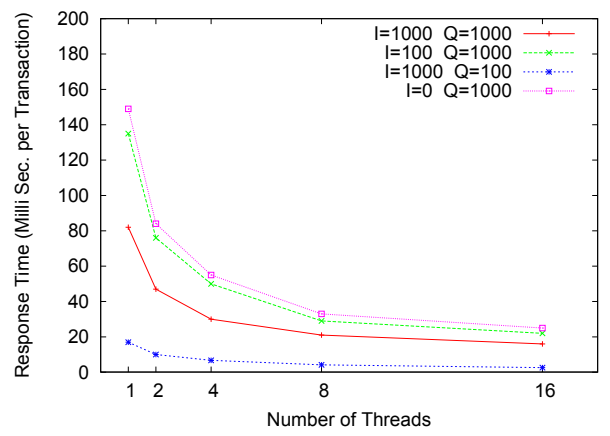

(a)

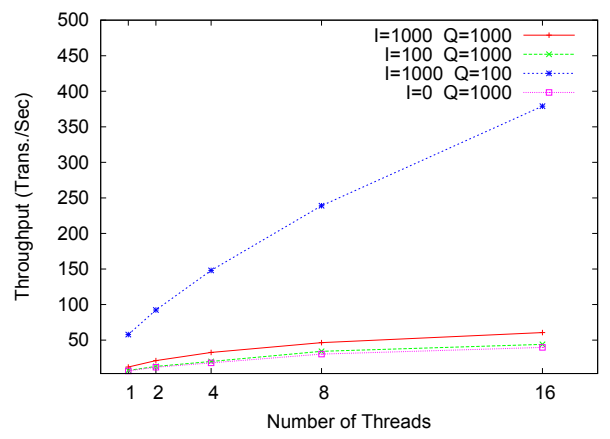

(c)

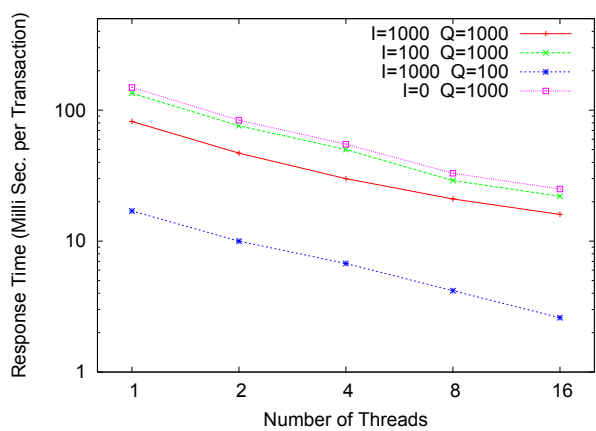

(b)

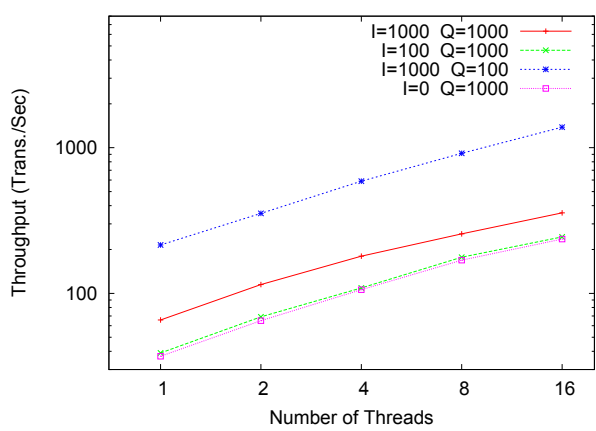

(d)

Figure 6.8: Parallel OLAP transaction performance of the PDC-Tree as a function of the number of parallel threads for Intel Sandy Bridge. Mixed input of $I$ OLAP insertions and $Q$ OLAP queries. Queries aggregate $5 \%$ of database. (a) Average response time, linear scale. (b) Average response time, log-log scale. (c) Throughput, linear scale. (d) Throughput, $\log -\log$ scale.

our case, the $P D C$-tree achieves a good speedup relative to a highly optimized and widely used public domain system.

Experiments on Intel Xeon Westmere EX: As discussed in the previous section, the Intel Sandy Bridge platform is very limited in terms of memory (i.e. database size) and number of cores available. In order to test our PDC-tree on an architecture that would be more typical for commercial in-memory OLAP systems, IBM Canada provided us access to one of their commercial servers, a 20 core Xeon Westmere EX with 256 GB memory. This allowed us to use a more realistic database size and also test the speedup achieved by our PDC-tree method for a larger number of processor cores. The latter is critical since achieving good speedup is typically easier for a smaller number of processors and becomes harder as the number of processor cores increases. We performed the following 


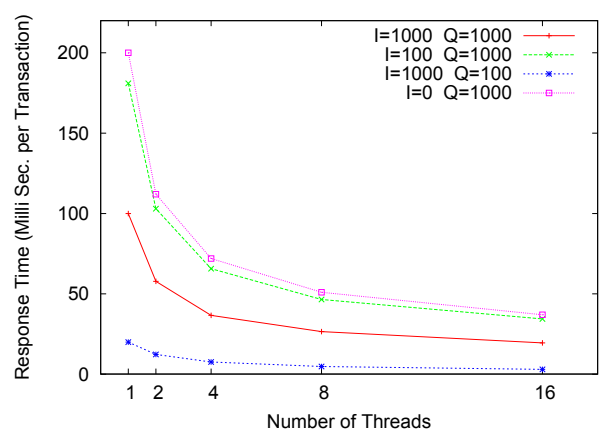

(a)

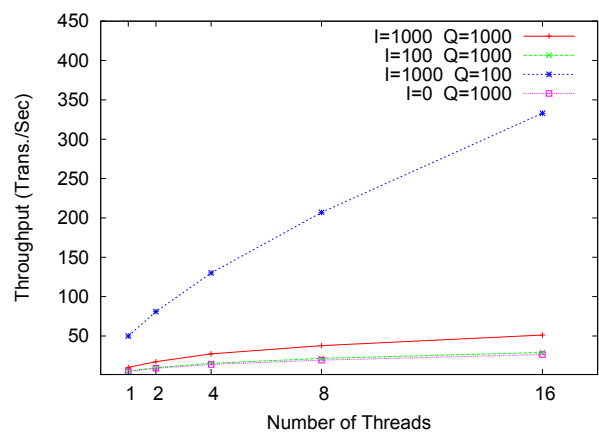

(c)

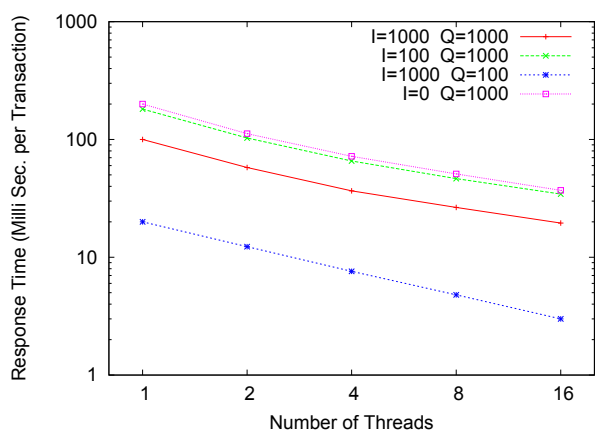

(b)

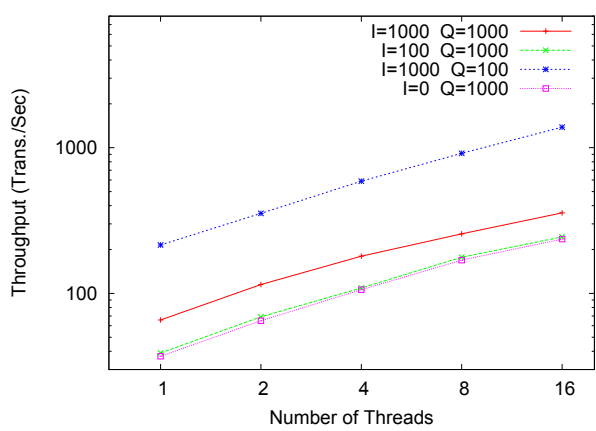

(d)

Figure 6.9: Parallel OLAP transaction performance of the PDC-Tree as a function of the number of parallel threads for Intel Sandy Bridge. Mixed input of I OLAP insertions and $Q$ OLAP queries. Queries aggregate $25 \%$ of database. (a) Average response time, linear scale. (b) Average response time, log-log scale. (c) Throughput, linear scale. (d) Throughput, log-log scale.

experiments:

1. Measuring the performance of 14 million OLAP inserts into a PDC-tree with 1 million data items (PDC-tree loading).

2. Measuring the performance of a mixed stream of OLAP insert and query transactions on a $P D C$-tree with 1 million data items.

3. Measuring the performance of a mixed stream of OLAP insert and query transactions on a PDC-tree with 10 million data items (100 GB).

The first experiment tests the performance of our $P D C$-tree implementation during the initial loading of a database. We built an initial PDC-tree with 1 million TPC-DS data 
Table 6.2: Runtime comparison between PDC-Tree and multithreaded MySQL. Times shown are the total runtimes for the same sequences of 1,000 OLAP queries, with varied query coverages, on the same databases with 400,000 items. All data and queries are from the TPC-DS benchmark.

\begin{tabular}{lllll}
\hline \hline PDC-Tree & & & \\
\hline \# Threads: & 1\% Coverage & 25\% Coverage & $75 \%$ Coverage & $100 \%$ Coverage \\
\hline 1 & $26.894 \mathrm{sec}$ & $197.745 \mathrm{sec}$ & $274.416 \mathrm{sec}$ & $9.136 \mathrm{sec}$ \\
2 & $15.395 \mathrm{sec}$ & $112.229 \mathrm{sec}$ & $156.912 \mathrm{sec}$ & $6.824 \mathrm{sec}$ \\
4 & $9.433 \mathrm{sec}$ & $72.014 \mathrm{sec}$ & $100.114 \mathrm{sec}$ & $5.433 \mathrm{sec}$ \\
8 & $5.942 \mathrm{sec}$ & $51.634 \mathrm{sec}$ & $72.265 \mathrm{sec}$ & $4.961 \mathrm{sec}$ \\
16 & $4.220 \mathrm{sec}$ & $37.892 \mathrm{sec}$ & $53.091 \mathrm{sec}$ & $4.788 \mathrm{sec}$ \\
\hline \hline MySQL & & & & \\
\hline \# Threads: & $1 \%$ Coverage & $25 \%$ Coverage & $75 \%$ Coverage & $100 \%$ Coverage \\
\hline "maximum" & $5 \mathrm{sec}$ & $161 \mathrm{sec}$ & $245 \mathrm{sec}$ & $415 \mathrm{sec}$ \\
\hline \hline
\end{tabular}

items and then executed a stream of 14 million TPC-DS data insertions. The measured throughput and transaction response times for the 14 million insertions are shown in Figure 6.10. The speedup shown for up to 20 threads (on 20 processor cores) is very close to optimal linear speedup. As outlined in Section 6.2.5, at any point in time, a PARALLEL_OLAP_INSERT operation only locks at most two PDC-tree directory nodes (e.g. for a node split). When the $P D C$-tree is large, there is close to no interference and parallel PARALLEL_OLAP_INSERT threads can operate independently on the tree data structure. This explains the very close to optimal speedup. As shown in Figure 6.10, when the number of threads is between 20 and 40, the additional speedup decreases because these additional threads are supported by hyper-threading rather than actual cores.

The second experiment tests the performance of our PDC-tree implementation for a mixed stream of OLAP insert and query operations on a PDC-tree for a smaller size database (but larger than the database on the Intel Sandy Bridge). We first built an initial PDC-tree by inserting 1 million rows of the TPC-DS data set and then executed a stream of TPC-DS transactions that consists of a mix of 1,000 query and 100 insert operations. Figure 6.11 shows throughput and response time of our PDC-tree implementation as we increase the number of threads. The curves show the performance for OLAP queries 


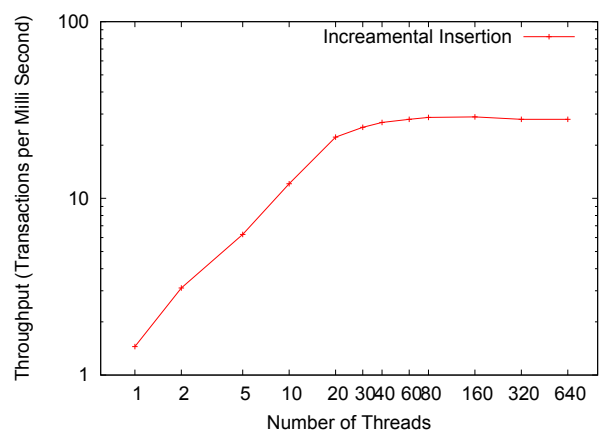

(a)

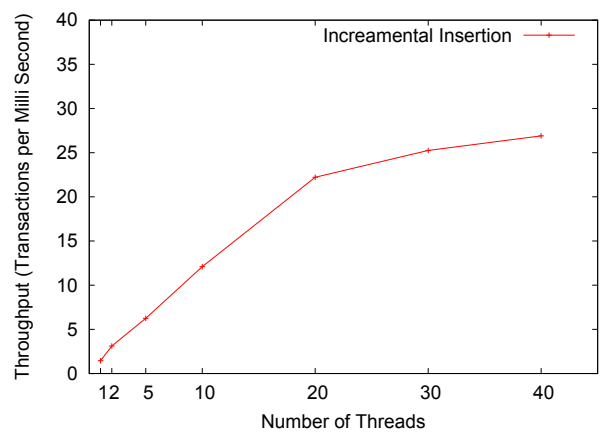

(c)

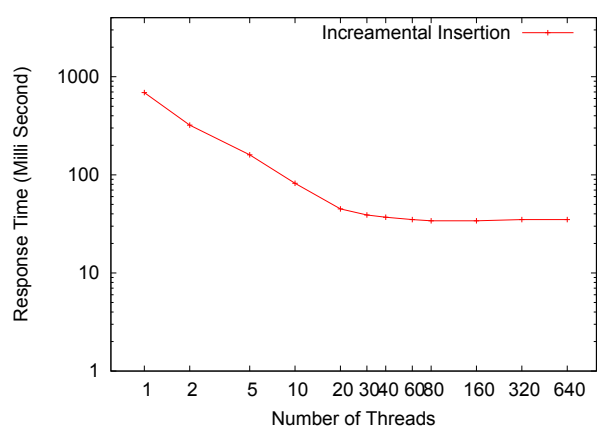

(b)

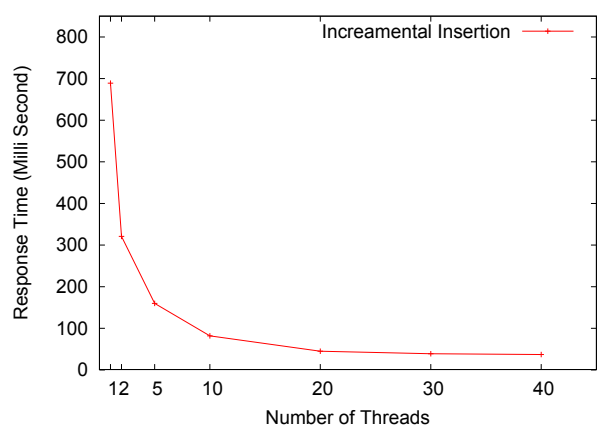

(d)

Figure 6.10: PDC-tree insertions only performance as a function of the number of parallel threads for Intel Xeon Westmere EX. Stream of 14 million insertions into an initial PDCtree with 1 million data items. (a) Throughput in log-log scale. (b) Average transaction response time in log-log scale. (c) Throughput in linear scale. (d) Average transaction response time in linear scale.

with $5 \%$ and $25 \%$ query coverage indicated in the figure. As shown in Figure 6.11, performance increases with close to optimal linear speedup for the range of 1 to 20 threads (on 20 processor cores). For the range of 20 to 40 threads, we obtain another $18 \%$ performance increase through hyper-threading which is within the typical 15\%-30\% interval reported by Intel[85]. Performance peaks at around 40 threads. For more than 40 threads, performance stays near flat (no more gains via latency hiding compared to overhead from context switching between threads). Note that after 40 threads the throughput/response time still varies slightly due to the overhead caused by thread context switching. In fact, the number of context switching increases by increasing the number of threads up to 640 threads. However, this impact is not considerable compared to the throughput gained by increasing number of threads. 


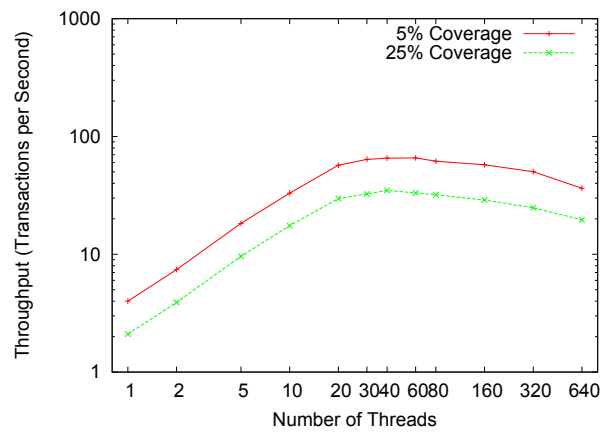

(a)

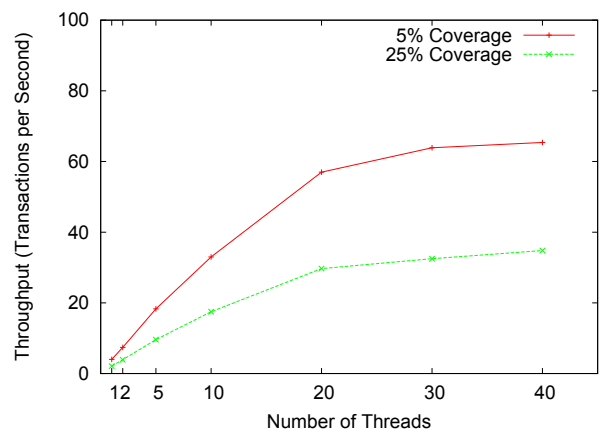

(c)

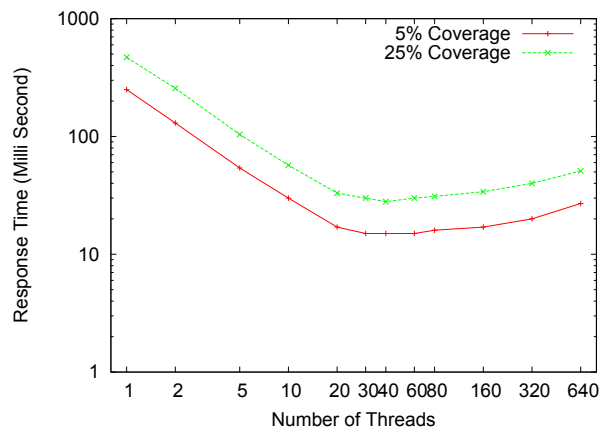

(b)

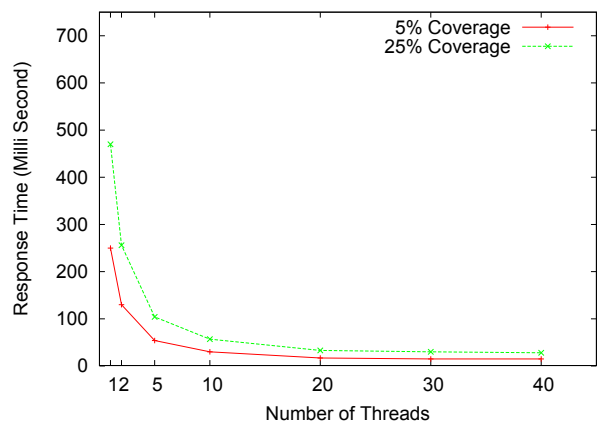

(d)

Figure 6.11: Parallel OLAP insertion and query performance of the PDC-Tree as a function of the number of parallel threads for two query coverages, $5 \% \& 25 \%$, on a PDC-tree with 1 million data items for Intel Xeon Westmere EX. Number of queries and insertions are 1000 and 100, respectively. (a) Throughput in log-log scale. (b) Average transaction response time in log-log scale. (c) Throughput in linear scale. (d) Average transaction response time in linear scale.

The third experiment tests the performance of our $P D C$-tree implementation for a mixed stream of OLAP insert and query transactions on a $P D C$-tree for a larger size database. We first built an initial $P D C$-tree by inserting 10 million rows (100 GB) of the TPC-DS data set and then executed a stream of TPC-DS transactions that consists of a mix of 10,000 query and 1,000 insertion operations. Figure 6.12 shows throughput and response time of our $P D C$-tree implementation as we increase the number of threads. The curves show the performance for OLAP queries with $5 \%$ and $25 \%$ query coverages, as indicated in the figure. Note that, the response times in Figure 6.12 are approximately ten times the response times in Figure 6.11 because on the PDC-tree with 10 million data items, OLAP queries with 5\% and $25 \%$ query coverage aggregate ten times as much data as OLAP queries with $5 \%$ and 
25\% query coverage on a $P D C$-tree with 10 million data items. As discussed earlier, query times are significantly larger than insertion times and dominate the average transaction performance. As shown in Figure 6.12, performance on the larger size database increases with close to optimal linear speedup for the range of 1 to 20 threads (on 20 processor cores). For the range of 20 to 40 threads, we obtain again a smaller performance increase through hyper-threading that is within the typical range reported by Intel[85]. Performance peaks again at around 40 threads. For more than 40 threads, performance stays flat as expected. After 40, the threads consume a fixed number of CPU cores as their computing resource which is 40 cores. Therefore, it is expected that very little or zero speedup is obtained by increasing the number of threads to more than 40 . In fact there is even a degradation observed in the curves after 40 threads. As mentioned earlier, the overhead caused by thread context switching adds extra time to every transaction leading to a degradation in the total performance. The speedup obtained is further elucidated in Table 6.3 (same data is in Figure 6.12) which shows for our mixed stream of 10,000 queries and 1,000 insertions the average transaction response times and speedup for $5 \%$ and $25 \%$ query coverage. In both cases, we measure an optimal linear speedup of 20 for a machine with 20 processor cores. We also observe that our multi-core parallelization enables us to achieve a better than 0.25 second response time for Real-time OLAP queries that aggregate $25 \%$ of a 100 GB database. 


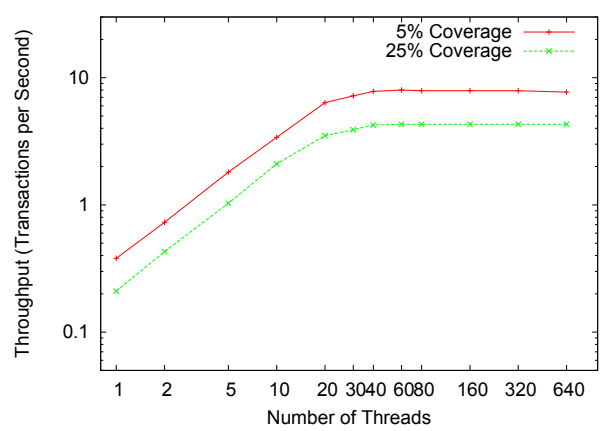

(a)

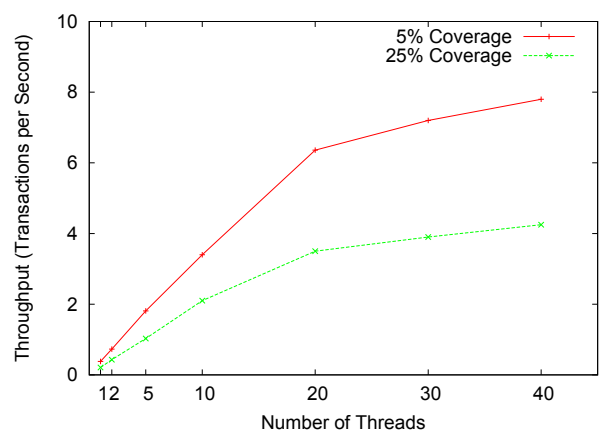

(c)

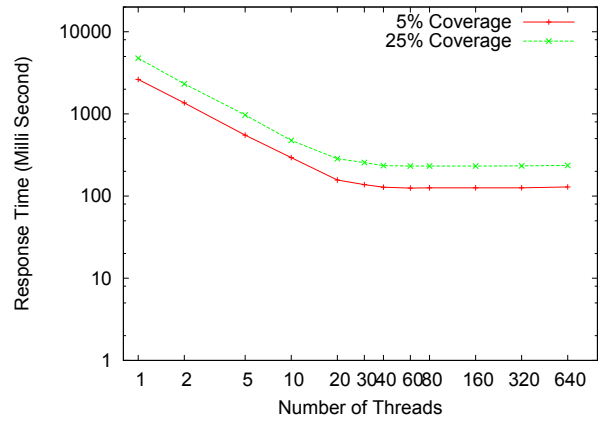

(b)

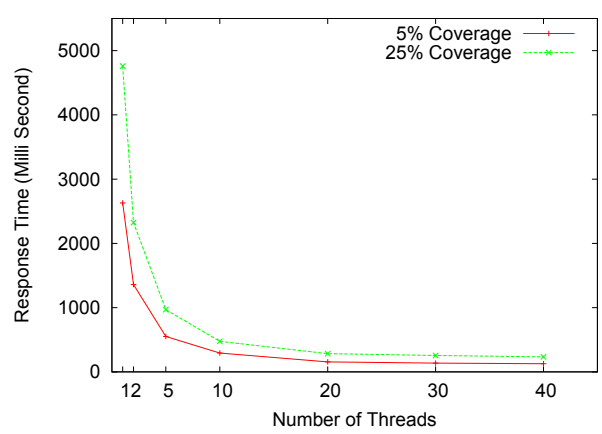

(d)

Figure 6.12: Parallel OLAP insertion and query performance of the PDC-Tree as a function of the number of parallel threads for two query coverages, $5 \%$ \& 25\%, on a PDC-tree with 10 million data items for Intel Xeon Westmere EX. Number of queries and insertions are 10,000 and 1000, respectively. (a) Throughput in log-log scale. (b) Average transaction response time in log-log scale. (c) Throughput in linear scale. (d) Average transaction response time in linear scale. 
Table 6.3: Parallel OLAP insertion and query performance of the PDC-Tree as a function of the number of parallel threads for two query coverages, $5 \% \& 25 \%$, on a PDC-tree with 10 million data items for Intel Xeon Westmere EX. Number of queries and insertions are 10,000 and 1000, respectively. Same data as in Figure 6.12. Average transaction response times and associated speedup.

\begin{tabular}{lllll}
\hline \hline Thread \# & $\begin{array}{l}\text { Response Time } \\
(\mathrm{sec}) \text { for 5\% } \\
\text { Query Coverage }\end{array}$ & $\begin{array}{l}\text { Speedup } \\
\text { for 5\% } \\
\text { Query Coverage }\end{array}$ & $\begin{array}{l}\text { Response Time } \\
(\mathrm{sec}) \text { for 25\% } \\
\text { Query Coverage }\end{array}$ & $\begin{array}{l}\text { Speedup } \\
\text { for 25\% } \\
\text { Query Coverage }\end{array}$ \\
\hline 1 & 2.47 & 1.00 & 4.57 & 1.00 \\
2 & 1.27 & 1.93 & 2.35 & 1.94 \\
5 & 0.51 & 4.77 & 0.94 & 4.82 \\
10 & 0.27 & 9.02 & 0.50 & 9.09 \\
20 & 0.14 & 16.80 & 0.28 & 16.25 \\
40 & 0.12 & 20.06 & 0.22 & 19.90 \\
80 & 0.12 & 20.48 & 0.22 & 20.05 \\
160 & 0.12 & 20.63 & 0.22 & 20.07 \\
320 & 0.12 & 20.39 & 0.22 & 19.95 \\
640 & 0.12 & 20.40 & 0.22 & 19.92 \\
\hline \hline
\end{tabular}




\subsection{Real-Time OLAP on Cloud Platforms}

\subsubsection{Statement of the Problem}

We phrase the addressed problem in this section as follows:

Assume a cloud environment with multi-core machines as cloud nodes; additionally assume a d-dimensional data warehouse with a fact table F; Develop a cloud solution including the required data structures and algorithms that enables a real-time OLAP system for the data warehouse in the cloud environment. The solution is expected to provide a scalable network of multi-core machines that simultaneously receive insertion and query transactions from clients. All the received transactions must be completed and responded in real-time according to the definitions given in Section 6.1.

\subsubsection{Introduction}

This section presents the last and most significant part of our parallel real-time OLAP project which is "to scale up our real-time OLAP system in a cloud environment". We introduce the $C R-O L A P$, a Cloud-based Real-time OLAP system that utilizes a new distributed index structure for OLAP, referred to as PDCR-tree. The PDCR-tree is a multi-dimensional data structure designed specifically to support efficient OLAP query processing on the elaborate dimension hierarchies for ordered and unordered dimensions.

The PDCR-tree is a non-trivial extension of the PDC-tree method to efficiently handle hierarchical data in different dimensions. It particularly provides a sophisticated structure to better handle the ordered and unordered dimensions of a data warehouse. The structure provides users with more flexibility compared to the $P D C$-tree in order to query the OLAP system on both ordered and unordered dimensions in different hierarchy levels. As such,

the PDCR-tree is not an R-tree. An important feature of the PDCR-tree is its storage scheme. Although the PDCR-tree is still a tree data structure, it is stored as an array so that it can be easily compressed and transferred between cloud machines via message passing. As the database grows and new workers are added to the system, the PDCR-tree splits up into multiple PDCR-trees in multiple workers, what is called a distributed PDCR-tree. The array-based storage scheme is not available for the $P D C$-tree since it is stored as nodes and links. 
The $C R-O L A P$ framework utilizes a collection of $(m+1)$ multi-core processors in a cloud environment. Consider a $d$-dimensional data warehouse with $d$ dimension hierarchies. The $C R-O L A P$ supports an input stream consisting of insert and query operations. Each OLAP query can be represented as an aggregate query that specifies for each dimension either multiple discrete values (for unordered dimensions) or multiple ranges of continuous values (for ordered dimensions) at any level of the respective dimension hierarchy. It also supports reporting the entire range for that dimension which implies the term "All" indicated by the "**" sign.

In the next section, the $C R-O L A P, P D C R$-tree, and related discussions are presented followed by a set of experiments for a variety of testing scenarios on the Amazon EC2 Cloud [1].

\subsubsection{CR-OLAP}

The $C R$-OLAP utilizes a cloud infrastructure consisting of $m+1$ multi-core processors where each processor executes up to $k$ parallel threads. As earlier discussed in this chapter for Real-time OLAP systems, all data is kept in the processors' main memories [89]. With increasing database size, the $C R-O L A P$ will increase $m$ by dynamically allocating additional processors within the cloud environment and re-arranging the distributed PDCRtree. This will ensure that both, the available memory and processing capability will scale with the database size. One of the $m+1$ multi-core processors is referred to as the master, and the remaining $m$ processors are called workers. The master receives from the users the input stream of OLAP insert and query operations, and reports the results back to the users. In order to ensure a high throughput and low latency even for compute intensive OLAP queries that may need to aggregate large portions of the entire database, the CR-OLAP utilizes several levels of parallelism:

1. Distributed processing of multiple query and insert operations among multiple workers

2. Parallel processing of multiple concurrent query and insert operations within each worker machine 
For correct query operation, the $C R-O L A P$ ensures that the result for each OLAP query includes all the data inserted before the query was issued but excludes later updates within the input stream. With increasing database size, the $C R$-OLAP will increase $m$ by dynamically allocating additional processors within the cloud environment and re-arranging the distributed PDCR-tree. This will ensure that both, the available memory and processing capability will scale with the database size.

The $C R-O L A P$ is supported by a new distributed index structure for OLAP termed the distributed PDCR-tree. The distributed index structure consists of a collection of PDCRtrees whereby the master stores one PDCR-tree (called hat) and each worker stores multiple PDCR-trees (called subtrees). Each individual PDCR-tree supports multi-core parallelism as the $P D C$-tree and executes multiple concurrent insert and query operations at any point in time. For a better understanding of a distributed PDCR-tree, imagine a single PDCR-tree $T$ storing the entire database. For a tunable depth parameter $h$, we refer to the top $h$ levels of $T$ as the hat and we refer to the remaining trees rooted at the leaves of the hat as the subtrees $s_{1}, \ldots, s_{n}$. The level $h$ is referred to as the cut level. We assume $n \geq m$ and that each worker stores one or more subtrees.

The $C R-O L A P$ starts with an empty database and one master processor (i.e. $m=0$ ) storing an empty hat which is a PDCR-tree. As in the PDC-tree, all data is stored in leaves called data nodes. Internal nodes are called directory nodes and contain arrays with routing information and aggregate values. As insert operations are sent to the $C R-O L A P$, the size and height of the hat (PDCR-tree) grows. When a directory node in the hat reaches a depth of $h$, its children are sent to the workers, and new subtrees are initiated with the children as the roots of the subtrees. The new workers are allocated through a request from the cloud environment by the the CR-OLAP. An illustration of such a distributed PDCR-tree is shown in Figure 6.13.

\section{Definition 3: distributed PDCR-tree}

A distributed PDCR-tree is a tree data structure containing two components:

1. Hat

2. A set $S$ of Sub-trees : $S=\left\{s_{1}, s_{2}, \ldots, s_{n}\right\}$ 


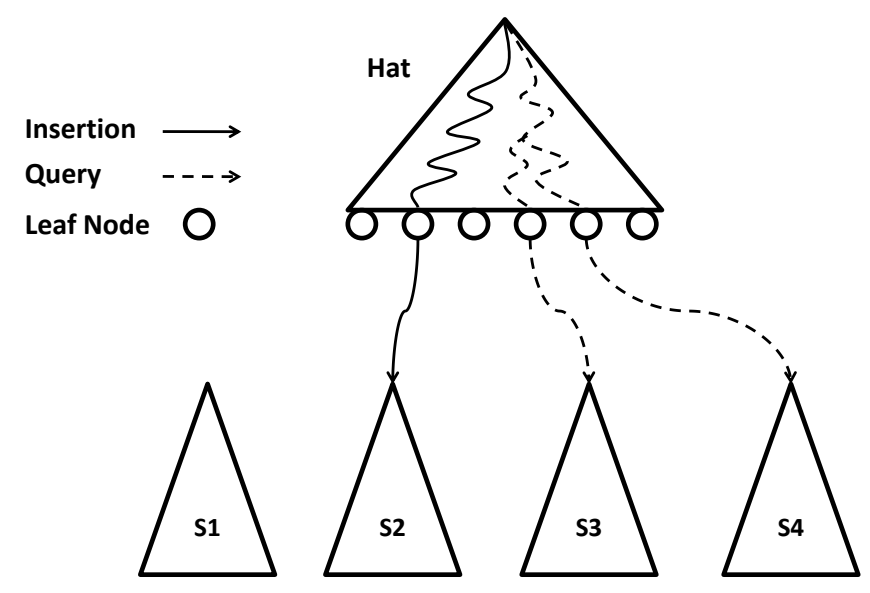

Figure 6.13: Illustration of a distributed PDCR tree.

Hat: The hat is a PDCR-tree containing the root of the tree and levels down to a depth of $h$. There are two types of nodes in the hat: directory nodes as internal nodes and data nodes as leaves. The hat through the master machine receives insertions and queries from clients and may dispatch them to one or a set of workers where necessary. In addition, the master organizes the workers and applies mechanisms for the scalability and load balancing of the cloud nodes. Workers may also communicate with the master to return the results of the queries. The master may keep communicating with clients to return the result of the aggregation queries. However, in case a client requests to receive part of the data in the tree rather than its aggregate value, the master allows workers to directly communicate with the client.

After the initial set of data insertions, all leaf nodes in the hat become directory nodes of height $h$. For a typical database size, the hat will usually contain only directory nodes and all data will be stored in the subtrees $s_{1}, \ldots, s_{n}$. In addition, the roots of the subtrees in the workers will typically be directory nodes as well. Each leaf directory node in the hat is the parent of many sub-trees with a capacity $c$, equal to the capacity of directory nodes in the tree.

Subtree: A subtree is a complete PDCR-tree containing the root of the subtree, directory nodes as internal nodes, and data nodes as the leaves of the subtree. Subtrees are the children of the leaf nodes in the hat. Therefore, the total number of subtrees in a distributed 


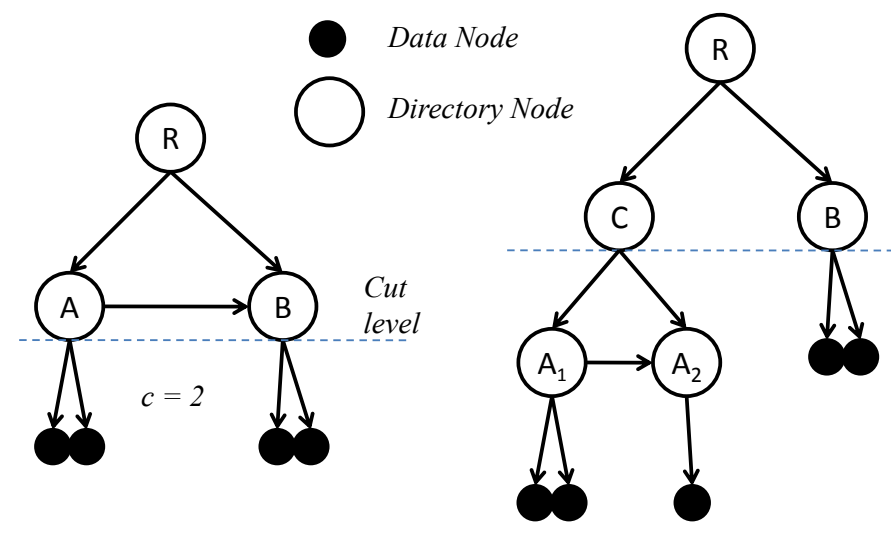

(a)

(b)

Figure 6.14: The Hat in the master. By the next insertion in node $A_{1}$, the link between nodes $A_{1}$ and $A_{2}$ will be removed. This releases the two nodes and both nodes are then moved to workers to initiate two new subtrees(Fig. 6.15).

PDCR-tree is equal to the number of the children of the leaf nodes in the hat. Figures 6.14 and 6.15 show the hat of a distributed PDCR-tree and the creation of new subtrees in two workers, respectively.

A complete process of building the hat and the initiation of the subtrees in the workers is depicted in Figure 6.16. The initiation of the new subtrees in this figure is triggered by the insertion of a data node under directory node $A_{1}$ (Figure 6.16(b)). Since $A_{1}$ exceeds the capacity of directory nodes $(c=2)$, it triggers a horizontal split in node $A_{1}$. In addition, since the parent and all ancestors of $A_{1}$ exceeded the capacity $(c)$, the horizontal split triggers a vertical split that creates node $D$ as the parent of $A_{1}$ and its new sibling node $A_{2}$ (Figure 6.16(c)). Locking mechanisms are applied to ensure proper read/write operations in nodes $A_{1}, A_{2}$, and $D$.

A key point in the vertical split process is that the horizontal link from node $A_{1}$ to node $A_{2}$ is no longer necessary, and it can be removed. This in turn removes the connection between the two nodes as well as their dependency on each other. The nodes become independent and may initiate two new subtrees. The hat then sends the two directory nodes with their children to the workers to initiate the new subtrees. As we will discuss it in the next sections, the new subtrees are sent to different workers to ensure a balanced distribution of the subtrees among the workers. 


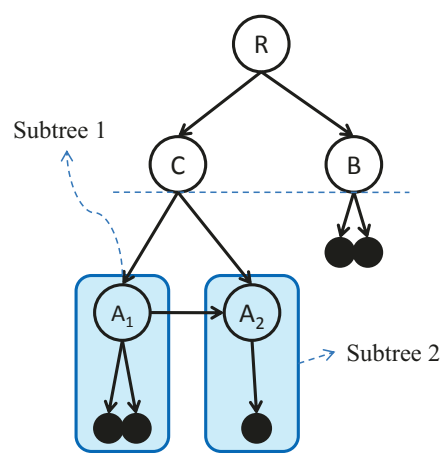

(a)

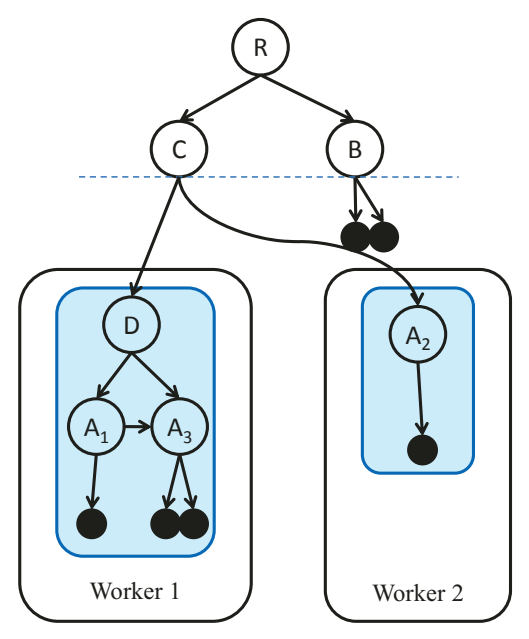

(b)

Figure 6.15: Initiation of two new subtrees in two workers. (a) All nodes are still in the Hat. (b) Last insertion occurred in node $A_{1}$ and caused a horizontal and vertical directory node split.

While removing horizontal links during a vertical split, threads can safely access sibling nodes or reprocess recently updated nodes. This is assured by using a stack mechanism independently for each thread to perform insertions and queries. This observation is proved through a set of theorems and lemmas. We present the correctness proofs in Appendix A and skip them here to keep a consistent flow of the context.

After the initiation of a new subtree in a worker, the insertions that are directed to the subtree are performed in two steps. First, the hat receives the insertion request from a client with the data record to be inserted. A thread picks the insertion transaction and traverses the hat starting with the root. For every directory node, enclosure of a data record in the directory node is tested using range checking. When the thread reaches the cut level of the hat, it determines in which subtree the data node must be inserted. It also reads a shared map including the list of all subtrees and workers to determine the worker machine that contains the subtree. The master then dispatches the insertion transaction to the specified worker. The worker receives the insertion transaction and inserts the data node in the target subtree. Figures 6.16(d-e) show the insertion of a new data node according to the described process. 


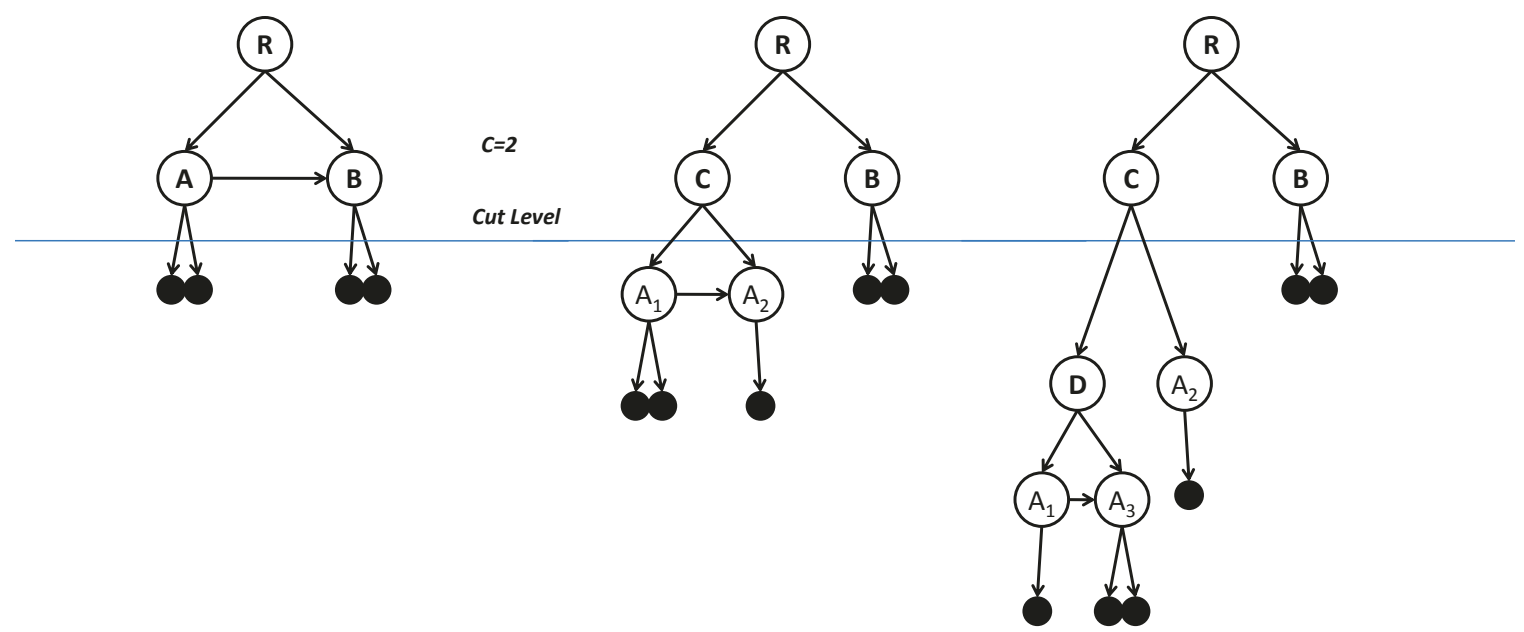

(a)

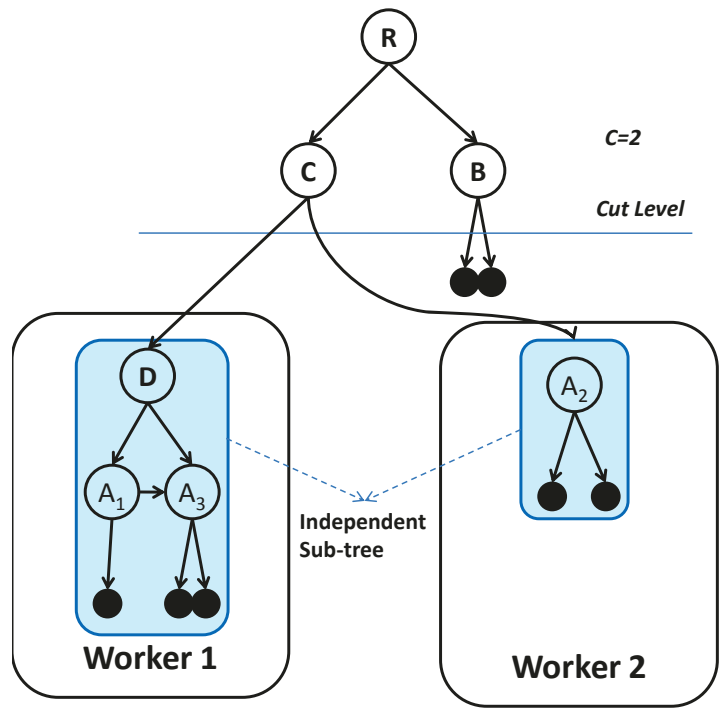

(d) (b)

(c)

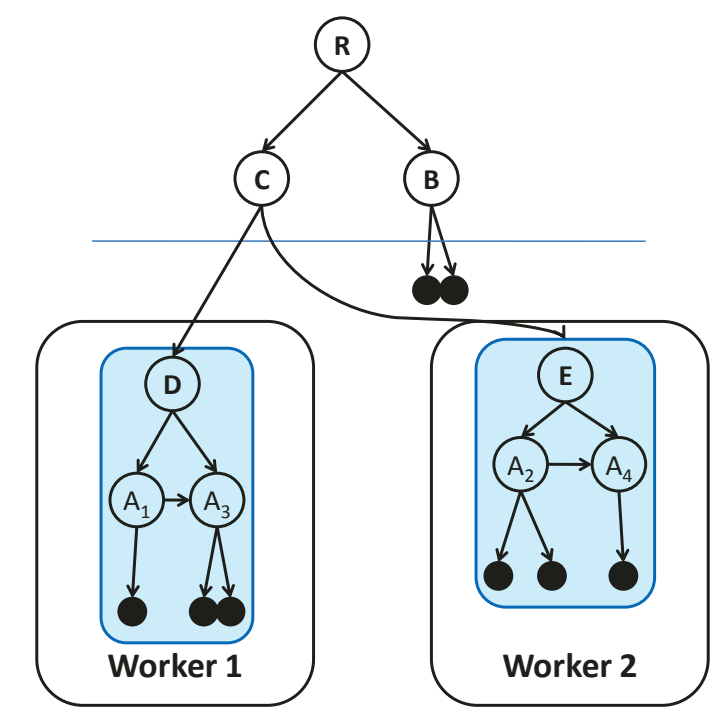

(e)

Figure 6.16: Growth of distributed PDCR-tree from an empty hat in the master and creation of subtrees in the workers. Solid black nodes represent data nodes and other nodes represent directory nodes. (a) The hat with 3 directory nodes in the master (no subtree/worker), (b) Insertion of a new data node and creation of a new directory node $(C)$. Node $B$ is now disconnected, however it is above the Cut Level, (c) Insertion of a new data node and creation of the directory node $D$. This case shows a transition stage. The depth of the hat has become greater than $h$. Subtrees $D$ and $A^{\prime}$ are now independent and may be sent to workers (Refer to Appendix A for proofs). (d) Two workers are allocated from the cloud environment by $C R-O L A P$, and subtrees are sent to the workers. (e) The subtrees in the workers grow by inserting new data records into distributed PDCR-tree. 
Figure 6.13 depicts a schematic picture of the distributed PDCR-tree after the sufficient number of insertions. As shown in Figure 6.13, the subtrees in the figure are distributed among the workers. An insertion transaction always traverses a single path in the hat and is dispatched to only one subtree. While a query transaction, depending on its coverage, may diverge to many paths in the hat and be dispatched to many subtrees in many workers. Note that this effect increases the parallelism by including multiple workers in the process.

After a sufficient number of insertions, there will be $n$ subtrees in the distributed PDCRtree, where $n \geq m$. The subtrees are evenly distributed among the workers in a round robin fashion to provide a balanced distribution of the subtrees and data nodes. The number of subtrees in the distributed PDCR-tree can be tuned using two parameters: directory node's $\operatorname{capacity}(c)$ and the depth of the hat $(h)$.

There are $c^{h}$ subtrees in the tree equal to the number of children of the leaf nodes in the hat. It is best optimized to set $n>>m$, since it provides us with a finer granularity in subtree sizes. A finer granularity facilitates load balancing by even distribution of the subtrees among the workers. Other advantages of this mechanism will be discussed in Section 6.3.6.

In the next section, we present the algorithms of the distributed PDCR-tree for insertion and query transactions in the master and workers.

\subsubsection{PDCR-tree}

Assume a data warehouse with a fact table $F$ and a set of $d$ dimensions as $\left\{D_{1}, D_{2}, \ldots, D_{d}\right\}$ where each dimension $D_{i}, 1 \leq i \leq d$ has a hierarchy $H_{i}$ including hierarchical attributes corresponding to the levels of the hierarchy. The hierarchical attributes in the hierarchy of dimension $i$ are organized as an ordered set $H_{i}$ of parent-child relationships in the hierarchy levels, i.e. $H_{i}=\left\{H_{i 1}, H_{i 2}, \ldots, H_{i l}\right\}$ where a parent logically summarizes and includes its children. Figure 6.17 shows the dimensions and hierarchy levels of each dimension for a 4-dimensional data warehouse.

In such a data warehouse, the $P D C$-tree partitions the $d$-dimensional data space into $d$-dimensional sub-spaces by defining hierarchies and partial ordering. There are two major differences between the DC-tree and the PDC-tree in one side with R-trees in the other 


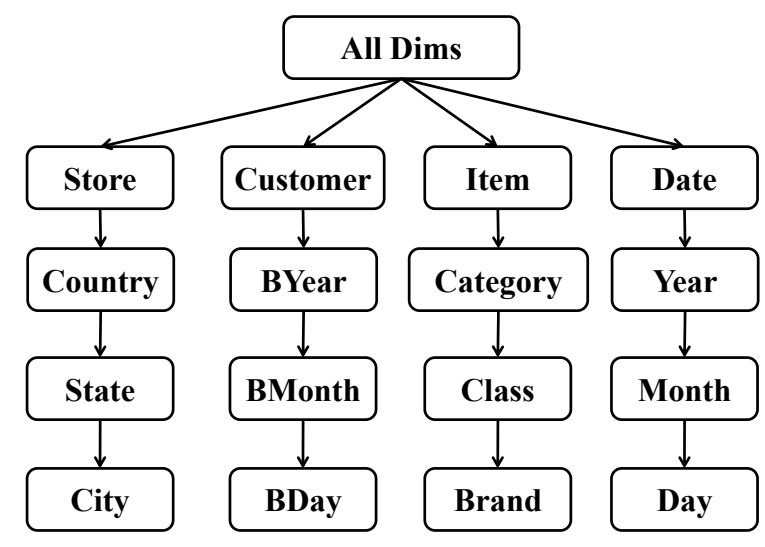

Figure 6.17: A 4-dimensional data warehouse with 3 hierarchy levels for each dimension. The first box for each dimension denotes the name of the dimension.

side. First, an R-tree may not index hierarchical information of the data in a data warehouse. This is an important drawback for R-trees since OLAP queries intensively require an OLAP system to answer the queries at different hierarchy and summarization levels. This requires the OLAP system to enable the OLAP operations such as roll-up, drill-down, slice, and dice. R-trees are not able to perform these OLAP operations since they do not index hierarchical data.

The second difference is in the way the trees index sub-spaces. An R-tree uses ranges for each dimension to specify the range of values that a subtree in the R-tree indexes. This method is effective for the dimensions that can be represented with ordered values known as ordered dimensions. Examples are Date and Time dimensions. However, a data warehouse often contains unordered dimensions such as Location, Products. Such dimensions may not be directly indexed by an R-tree, particularly if the dimension includes hierarchical information that can be requested by the OLAP queries. As discussed in Section 6.2, the DC-tree and PDC-tree overcome this issue by applying a set of distinct hierarchy values called Minimum Describing Sequence (MDS) for every dimension in the subtrees of the tree.

The PDC-tree indexes dimensions of a data warehouse by defining partial ordering for each dimension and using MDS to describe a summarization for underlying data. However, the partial ordering is applied to all dimensions disregarding whether a dimension is ordered or unordered. This poses serious problems particularly when the MDS is used to 
represent a range in an ordered dimension. The challenge is how a range of continuous values can be represented by an MDS. In fact, MDS is originally built for discrete values, and causes performance issues when representing ranges. To address this issue, we present PDCR-tree, a new multi-dimensional tree that can index ordered and unordered dimensions in the proper way. The PDCR-tree treats ordered dimensions with multiple ranges of values known as Multiple Minimum Bounding Rectangles (MMBR), and it treats unordered dimensions with a set of discrete values similar to the MDS. Note that an MDS is able to represent multiple hierarchical values.

The PDCR-tree provides mentioned features by using IDs as the key elements of every MMBR and MDS. In the following, we describe the structure of the IDs followed by a discussion on the query types that can be answered in the PDCR-tree.

IDs: In a $d$-dimensional data warehouse, IDs for each dimension represent available entities in the dimension. Considering the definition of a data warehouse presented earlier in this section, each dimension has a hierarchy of entities with $l$ levels. Each ID represents an entity in one of the $l$ hierarchy levels. In the example of Figure 6.17, an ID may represent an entity in the "country" level for the Store dimension (e.g. US, Canada). Similarly, another ID may represent an entity in the "city" level (e.g. Chicago, Toronto). There are three rules that apply to all IDs:

1. Each ID represents an entity of only one dimension.

2. Each ID represents only one entity in its corresponding dimension. The entity may logically summarize many IDs in a lower hierarchy level.

3. Each ID represents an entity only in one hierarchy level, although it contains information about all ancestor entities in the hierarchy of its corresponding dimension.

As an example, the ID for the Toronto value in the Store dimension, specifies the entity Toronto which is located in the province of Ontario in the country of Canada. Information about the ancestor values in the hierarchy helps later in the indexing of data and directing queries. Figure 6.18 illustrates the structure of an ID with an example for a store entity in the Store dimension. 
To build an ID for a dimension, we assign $b_{j}$ bits to the hierarchy level $j, 0 \leq j \leq l-1$ in the dimension. Different entities in every hierarchy level are assigned with numerical values starting from 1. By concatenating the numerical values of the levels, a final numerical value is created. We reserve the value zero to represent the concept "All" (i.e. “*”) covering all entities in each level.

The ID sample in Figure 6.18 represents an entity in the lowest hierarchy level of the Store dimension. However, IDs can represent entities in all hierarchy levels of a dimension. An ID for California will have a value of zero for its descendant levels (i.e. City and Store $S \_$key). As a result, the enclosure of an ID for different hierarchy levels can be tested using bound checking or bitwise operations. Figure 6.19 illustrates IDs and their coverages in the Store dimension with respect to the hierarchy levels. As shown in this figure, each entity in the level $j$ (i.e. Country) is a country specified by a numerical value. Each numerical value in the Country level covers cities that are represented using numerical values in level $j+1$. Note that the IDs used for the cities will have specific values in the city level, while the ID of a country will have a value of zero in the city level and a specific value in the country level.

Such representation in fact provides us with the advantages of the PDC-tree and its MDS representation which help to summarize all lower level entities in a single higher level entity. The ID structure can effectively help us in indexing unordered dimensions where there is no range. In fact, IDs provide a $d$-dimensional data representation from both a hierarchical view and a range view. On the other hand, for ordered dimensions, we can still apply the concept of ranges by simply providing lower and upper bounds in the corresponding hierarchy level.

The proposed ID representation offers an important advantage: using a single ID we can represent all similar entities in a hierarchy level $l$ that belong to different entities in the level $l-1$. To illustrate this feature, consider the Date dimension in Figure 6.17. Using the proposed ID structure, a single ID can represent the sub-space "February of all years”. Such an ID is set to its corresponding dimension value in level 0 . Level 1 , representing the year in the ID is set to zero, and level 2 representing the month is set to 2. The created ID can be used in a query that covers "Total sales in the February of all years". Note that such queries will be executed in the tree only one time for all years 


\begin{tabular}{|c|c|c|c|c|}
\hline Level 0 & Level 1 & Level 2 & $\ldots$ & Level l \\
\hline Store & US & California & Los Angels & Store S_key \\
\hline b $\longrightarrow$ bits $\longrightarrow \mathrm{b}_{1}$ bits $\longrightarrow$ bits $\longrightarrow$ \\
\hline 01 & 01 & 1001 & 101 & 1011011 \\
\hline
\end{tabular}

Figure 6.18: The structure of an ID. The bits in each level are used to represent values for the entity and its ancestors in the upper hierarchy levels. The sample ID below represent a unique store located in the city of Los Angeles, in California, in the US. Note that concatenation of the bits in the levels builds up a large unique numerical value that represents the corresponding store.

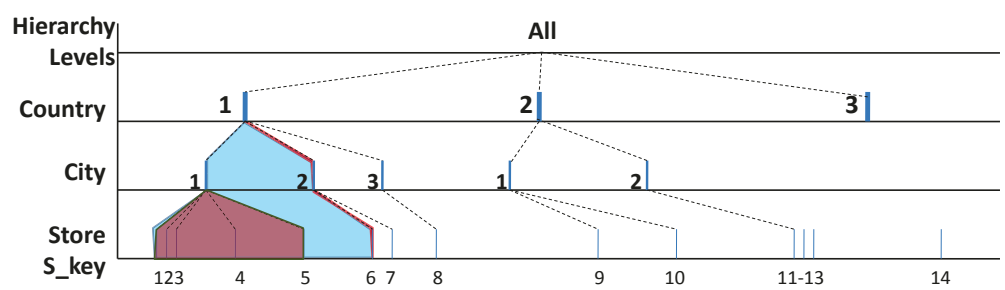

Figure 6.19: The structure of IDs for the Store dimension with their coverages. Note that city values for each country starts from 1 , while Store $S \_$keys are unique surrogate keys in the dimension. Lightly shaded area shows the coverage of the country 1 , while the dark shaded area is the coverage of the city 1 in country 1 .

rather than multiple executions for different years. This is a considerable advantage of the ID representation over other methods where such queries have to be divided into multiple queries. In addition, we can combine this feature with the ranges. For example, by using two IDs as the lower and upper bounds in the previous example, we can specify a range of entities in the month level for all years (e.g. "February-May of all years").

The features provided in the ID representation enable the PDCR-tree to efficiently describe each dimension in the right manner by considering whether it is an ordered or unordered dimension. The ID structure enables us to represent sub-spaces that can be used in indexing subtrees as well as providing a variety of OLAP queries. In the following we describe the different types of the queries that a PDCR-tree may accept.

Queries: In a $d$ dimensional data warehouse described earlier in this section, queries with 
the following templates can be answered by the PDCR-tree: Query: $\left\{Q_{1}, Q_{2}, \ldots, Q_{d}\right\}$ where $Q_{i}$ with $1 \leq i \leq d$ represents a set of aggregated values (MDS) or multiple ranges (MMBR) for dimension $i$ to be searched/queried. The set $Q_{i}$ is represented by either of the followings:

1. Multiple ranges of values (MMBR) each covering a continuous range of values in a hierarchy level of dimension $i$. This type of query values is used only for the ordered dimensions such as date, time.

2. Multiple MDS IDs for any level of the hierarchy of dimension $i$. Each MDS ID in the hierarchy level $j, 0 \leq j \leq l-1$ in dimension $i$, covers many discrete sub-spaces in level $j+1$ of the dimension. This type of query values can be used for both ordered and unordered dimensions such as Location, Product, Date.

Considering the definitions of the ID, the PDCR-tree can answer two types of queries as follows:

1. Range queries for ordered dimension $i$ :

$Q_{i}=\left\{\left[\right.\right.$ lowerID $_{i 1}$, upperID $\left._{i 1}\right],\left[\right.$ lowerID $_{i 2}$, upper $\left.-I D_{i 2}\right], \ldots,\left[\right.$ lowerID $_{\text {in }}$, upperID $\left.\left._{\text {in }}\right]\right\}$ , where lowerID $D_{i j}$ represents the lower bound of the $j t h$ given range in dimension $i$. UpperID $i j$ represents the upper bound of the $j t h$ given range in dimension $i$.

2. Point queries for ordered/unordered dimension $i$ : $Q_{i}=\left\{I D_{i 1}, I D_{i 2}, I D_{i n}\right\}$, where $I D_{i j}$ represents the $j t h$ given MDS ID in dimension $i$. Note that the IDs can be in the different hierarchy levels of dimension $i$ (e.g. an ID representing the month February of the year 2013).

Building PDCR-tree: In order to build a PDCR-tree, we use the ranges in the IDs to index data in each dimension regardless of whether it is ordered or unordered. Figure 6.20 shows a sketch of how a PDCR-tree indexes data in a very small data warehouse with 2 dimensions Store and Date. In this figure, both ordered dimension (i.e. Date) and unordered dimension (i.e. Store) are treated as ranges. Note that regardless of indexing unordered dimensions as ranges, queries may still include unordered aggregate values on the Store dimension (e.g. "Total sales in the US and Canada" or "Total sales in California and New York"). In fact, the PDCR-tree uses ranges only to index the data in both ordered and unordered dimensions. 


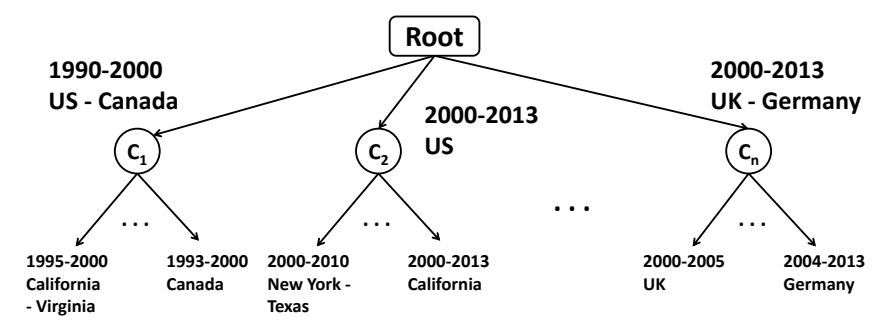

Figure 6.20: A simple PDCR-tree with 2 dimensions Store and Date. Note both ordered and unordered dimensions can be treated as ranges, depending on the applied multi-dimensional indexing algorithms.

However, due to the hierarchical structure of IDs, the point queries for the different hierarchy levels can be answered efficiently as well as range queries. In addition, we found range checking to be more efficient than bit-wise operations inside the IDs for enclosure tests.

\subsubsection{Algorithms}

In this section we present four algorithms of the distributed PDCR-tree for data node insertion and query processing.

\subsubsection{Insertion Algorithms}

In Section 6.3.4, we discussed the insertion process in the distributed PDCR-tree. Algorithm 4 (Hat_Insertion) is a pseudo code of the Insertion Algorithm in the hat. Upon receiving an insertion request in the master, the $C R-O L A P$ assigns a thread to the transaction. The thread starts Algorithm 4 for the insertion transaction.

In case the hat is full, the data node is sent to a subtree in a worker. The worker receives the insertion transaction and assigns the transaction to a thread for processing. The thread calls Algorithm 5 (Subtree Insertion) to insert the data node in the target subtree.

\subsubsection{Query Algorithms}

Query processing in the distributed PDCR-tree includes two stages similar to the insertion processing: a hat step and a following subtree step. Similar to the insertion process, query 


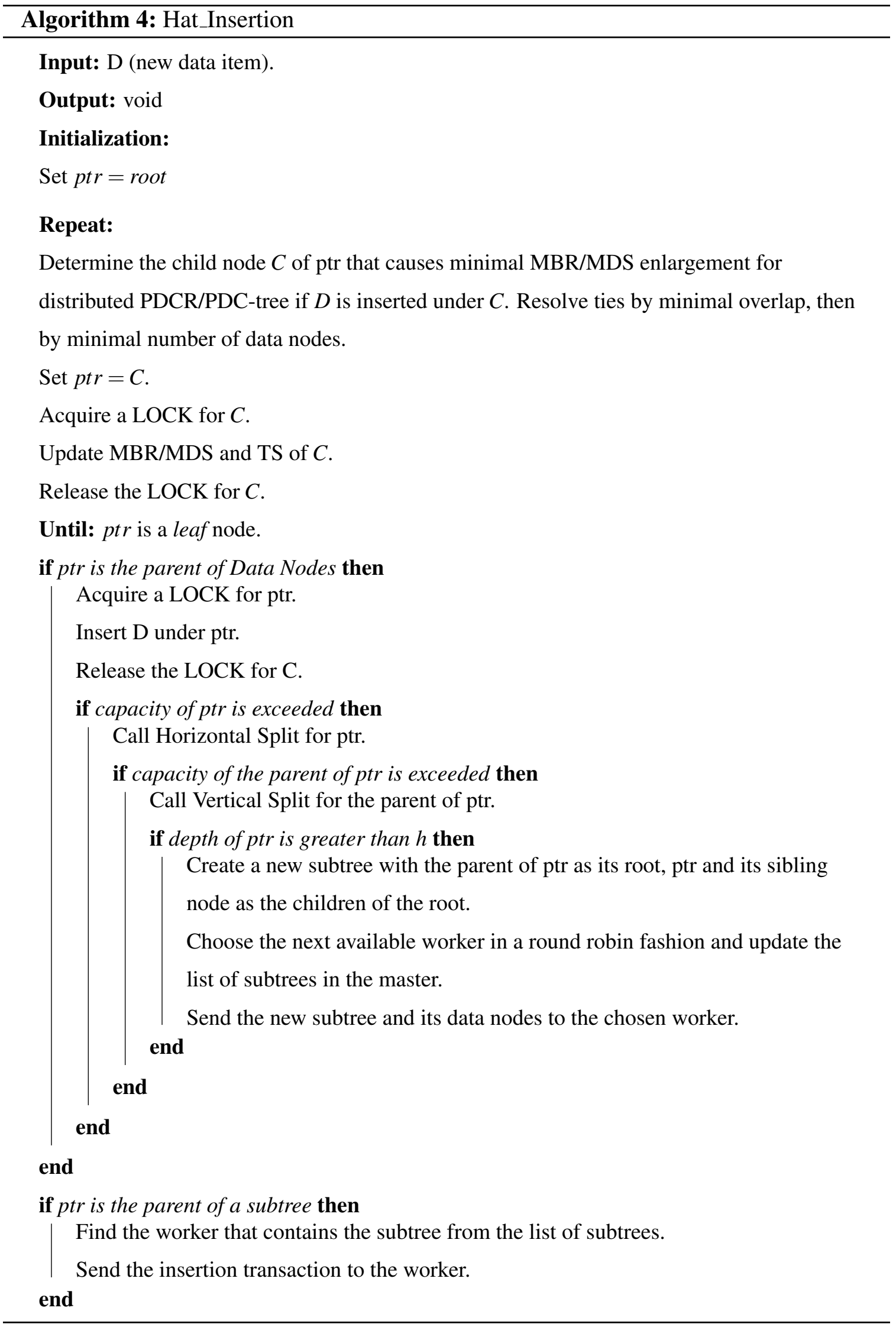




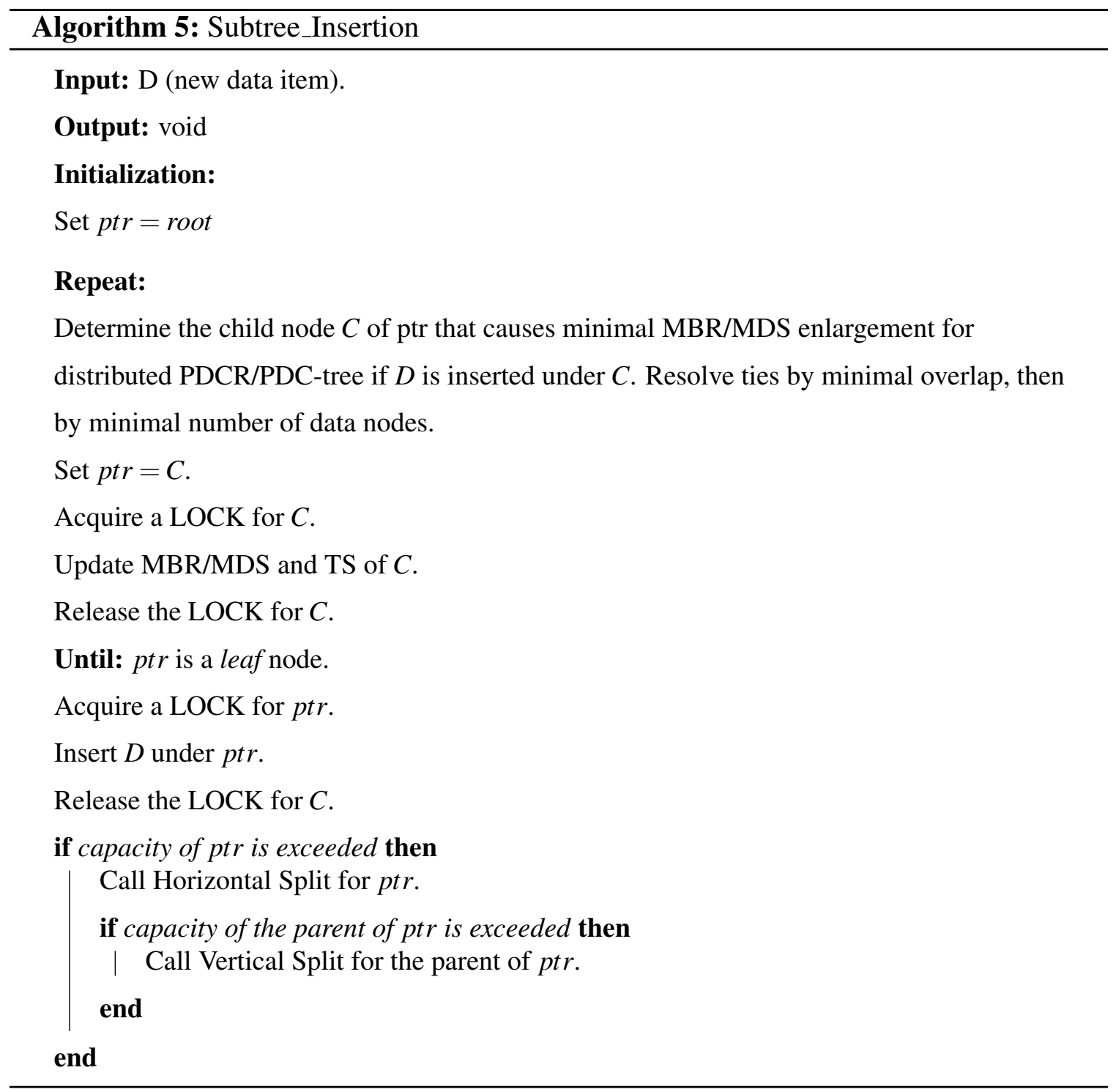


processing starts by traversing the hat from its root and seeking the directory nodes that are fully or partially covered by the query. The query process adds directory nodes that are fully covered to the result set without further processing. For directory nodes that overlap the query, their child nodes are pushed into a stack for further processing. This is similar to the query processing in the PDC-tree. The only difference with the PDC-tree's query processing is that the PDCR-tree uses range checking which we found to be a lighter process than the enclosure tests in the PDC-tree. If the query process reaches the cut level, the query transaction determines the subtrees that overlap with the query. Next, the query transaction is dispatched to the workers that contain the overlapping subtrees. Note that a query may cover/overlap many subtrees and may be sent to many workers. Whereas an insertion transaction is dispatched to only one subtree in one worker machine.

Another difference between an insertion and a query transaction is in returning the query result back to the client that issued the query. An insertion process does not return any result to the client while a query process is required to collect partial query results during the process and send the final aggregated result back to the client. To return the final result, the dispatcher thread in the hat waits for the subtrees until all partial results of the dispatched queries are returned to the master. The dispatcher thread then collects the partial results and creates the final result. Algorithms 6 and 7 are the pseudo codes of the query processes in the hat and subtrees, respectively.

It is important to note that PDCR-tree does not need to explicitly implement OLAP operations such as roll-up, drill-down, slice, and dice. In the next section, we discuss strategies and mechanisms in $C R-O L A P$ for scalability and load balancing.

\subsubsection{Scalability and Load Balancing}

The $C R-O L A P$ is executed on a cloud platform with $(m+1)$ processors ( $m$ workers and one master). As discussed earlier, the CR-OLAP uses the cloud's elasticity to increase $m$ as the number of data items increases. We now discuss in more detail the CR-OLAP's mechanisms for worker allocation and load balancing in the cloud. The insert operations discussed above create independent subtrees for each height $h$ leaf of the hat. Since directory nodes have a high capacity (c) (typically 10 - 20), a relatively small height of the hat leads to thousands of height $h$ leaves and associated subtrees $s_{1}, \ldots, s_{n}$. The master 


Algorithm 6: Hat_Query

Input: Q (OLAP query).

Output: A result set or an aggregate value

\section{Initialization:}

Set $p t r=$ root Push $p t r$ into a local stack $S$ for query $Q$.

\section{Repeat:}

Pop the top item $p t r^{\prime}$ from stack $S$.

if TS(time stamp) of ptr' is smaller (earlier) than the TS of ptr then

Using the Link to Sibling, traverse the sibling nodes of ptr until a node with TS equal to the TS of $p t r$ is met. Push the visited nodes including ptr into the stack starting from the rightmost node for reprocessing.

\section{end}

for each child $C$ of ptr do

if $M B R / M D S$ of $C$ is fully contained in $M B R / M D S$ of $Q$ then

I Add $C$ and its measure value to the result set.

end

else

if $M B R / M D S$ of $C$ is overlaps $M B R / M D S$ of $Q$ then

if $C$ is the root of a sub-tree then

I Send the query $Q$ to the worker that contains the subtree.

end

else

I Push $C$ into the stack $S$.

end

end

end

end

Until: stack $S$ is empty.

if the query $Q$ is dispatched to a subtree then

Wait for the partial results of the dispatched queries from workers.

Create the final result of the collected partial results.

Send the final result back to the client.

end 


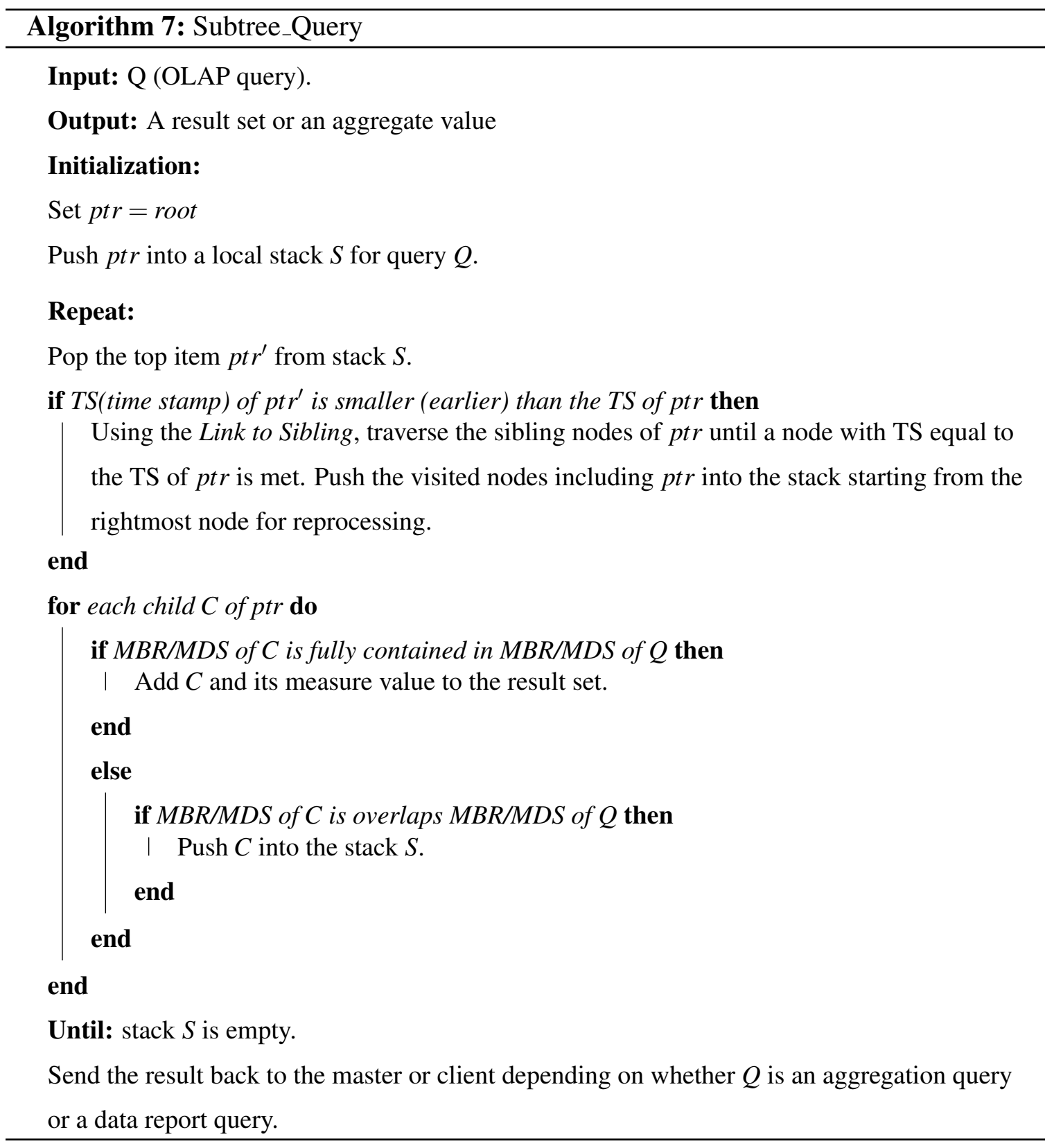


processor keeps track of the subtree locations and allocation of new workers, and it makes sure that a relatively high $n / m$ ratio is maintained. As indicated earlier, the CR-OLAP applies a round-robin fashion to distribute these $n>>m$ subtrees among the $m$ workers. This ensures that query operations in particular large OLAP queries are split into many smaller queries distributed over the workers. Furthermore, the CR-OLAP performs load balancing among the workers to ensure both, a balanced workload and memory utilization. The master processor keeps track of the current subtree sizes and the number of active threads for all subtrees. For each worker, its memory utilization and workload is recorded.

If a worker $w$ has a memory utilization above a certain threshold (e.g. $75 \%$ of its total memory), then the master processor determines the worker $w^{\prime}$ with the lowest memory utilization and checks whether it is possible to store an additional subtree from $w$ while staying well below its memory threshold (e.g. $50 \%$ of its total memory). If that is not possible, a new worker $w^{\prime}$ is allocated within the cloud environment. Then, a subtree from $w$ is compressed and sent from $w$ to $w^{\prime}$ via message passing. As discussed earlier, the PDCRtree is implemented in array format and using only array indices as pointers. This enables fast compression and decompression of subtrees and greatly facilitates subtree migration between workers. Similarly, if a worker $w$ has a workload utilization that is a certain percentage above the average workload of the $m$ workers and is close to the maximum workload threshold for a single worker, then the master processor determines a worker $w^{\prime}$ with the lowest workload and well below its maximum workload threshold. If that is not possible, a new worker $w^{\prime}$ is allocated within the cloud environment. Then, the master processor initiates the migration of one or more subtrees from $w$ (and possibly other workers) to $w^{\prime}$. Figure 6.21 depicts a schematic picture of our load balancing strategy.

\subsubsection{STREAM-OLAP}

A possible disadvantage of tree data structures is that they are potentially less cache efficient than in-memory linear search which can make optimum use of streaming data between memory and processor caches. To establish a comparison baseline for the CR-OLAP, we implemented STREAM-OLAP which partitions the database between multiple cloud processors based on one chosen dimension and uses parallel memory to cache streaming on the cloud processors to answer OLAP queries. The STREAM-OLAP is a 1-dimensional 


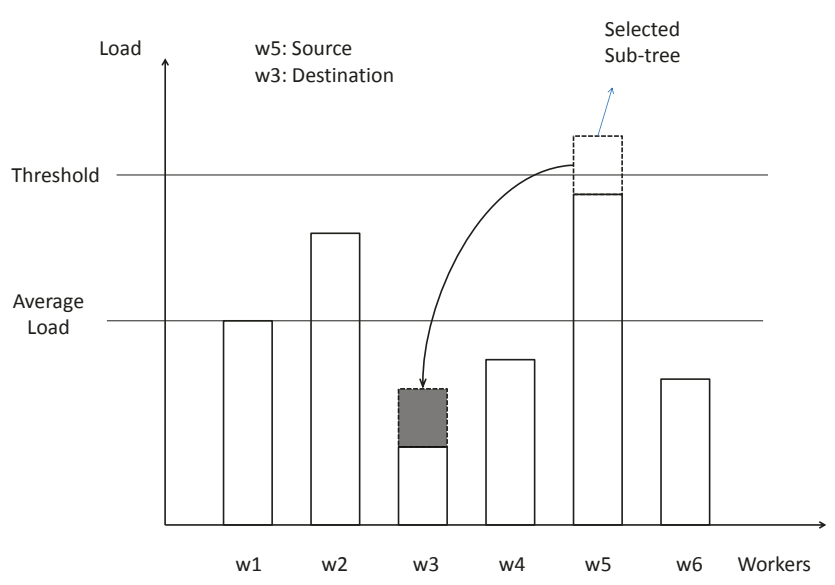

Figure 6.21: A schematic picture of load balancing strategy. Load in worker w5 is detected above threshold. A subtree in the worker is selected for migration to worker w3 as destination worker.

index that uses the same ID structure and enclosure tests as the PDCR-tree. The STREAMOLAP differs from the CR-OLAP in that it indexes data using one chosen dimension while the PDCR-tree uses all dimensions to index the data. The 1D-index uses the Customer dimension to index the data into many 1-dimensional arrays. Data nodes in each array are inserted at the end of the array as they arrive. The arrays are evenly distributed between the workers to assure a balanced parallel processing of the insertions and queries. In each array, multiple queries may search the array in parallel and return results.

The goal of the implementation of the STREAM-OLAP is to compare its performance against the CR-OLAP and evaluate the impact of a 1-dimensional index versus a multidimensional index for hierarchical data. Note that R-trees and B-trees are different from the PDCR-tree in that they do not index/query hierarchical data. Hence, they may not represent a comparison baseline for the PDCR-tree.

\subsubsection{Experimental Results}

\subsubsection{Setup}

In this section, we present the framework and setup used for testing the CR-OLAP on a cloud platform. In the next section, we present the results of our experiments on the 
Amazon EC2 Cloud.

Cloud Configuration and Setup: The CR-OLAP was executed on the Amazon EC2 Cloud. For the master processor we used an Amazon EC2 m2.4xlarge instance: "High-Memory Quadruple Extra Large" with 8 virtual cores (64-bit architecture, 3.25 ECUs per core) rated at 26 compute units and with $68.4 \mathrm{GiB}$ memory. For the worker processors we used Amazon EC2 m3.2xlarge instances: "M3 Double Extra Large" with 8 virtual cores (64-bit architecture, 3.25 ECUs per core) rated at 26 compute units and with $30 \mathrm{GiB}$ memory. The OS image used was the standard Amazon CentOS (Linux) AMI.

As discussed in Section 6.3.3, the CR-OLAP includes a master node and $m$ worker nodes. The master receives data from a remote machine outside of the cloud environment. The remote machine contains a repository of queries and a data warehouse including $d$ dimensions and a fact table $F$. The master machine in the cloud receives requests including insertions and queries, and wraps them as insertion/query transactions. The master then assigns the transaction with a thread. The thread then executes the transaction accordingly in the tree starting from the hat. We use tuples of the fact table for insertions, while for queries we use a set of pre-generated queries stored in the remote machine.

Implementation and Configurations: The $C R-O L A P$ was implemented in $\mathrm{C}++$, using the g++ compiler, OpenMP for multi-threading, and ZeroMQ [11] for message passing between processors. Instead of the usual MPI message passing library we chose ZeroMQ because it better supports cloud elasticity and the addition of new processors during runtime. The system runs on a Linux platform. The $C R-O L A P$ has various tunable parameters including depth of the hat $(h)$, directory node capacity $(c)$, the number of threads in each worker $(k)$, and the number of the subtrees in the workers. According to our optimization tests results, we summarize the optimal values of each parameter in Table 6.4.

For finding the optimal value for each parameter, we first loaded the $C R-O L A P$ with 40 million data records on 8 workers. Next for each parameter, we fixed other parameters at known optimal values, and started a set of repetitive tests for a range of different values of the testing parameter. We expanded the ranges until we assured that the response times are strictly diverging. Then we recorded the found optimal value for the testing parameter and updated our table of optimal values. The tests included a set of queries with mixed query 
Table 6.4: The optimal values of trees' parameters for the current Cloud setup.

\begin{tabular}{ll}
\hline Parameter & Optimal Value \\
\hline Depth of the hat (h) & 2 \\
Directory nodes' capacity (c) & 15 \\
Number of threads in each worker & 16 \\
Number of subtrees in the workers & 225 \\
\hline
\end{tabular}

coverages and a set of insertions. For the first testing parameter, since there was no found optimal value at that time, we used optimal values that we found based on experience at the implementation time.

Test Data Set: For our experimental evaluation of the CR-OLAP and STREAM-OLAP, we used the standard TPC-DS benchmark for OLAP systems [109]. The TPC-DS benchmark generates a database of 7 fact tables and 10 dimensions. We use the fact table "Store Sales" in our experiments. The "Store Sales" is the largest fact table in the 7 fact tables in terms of size. The TPC-DS provides a stream of insert and query operations on the "Store Sales" which was used as the input for the CR-OLAP and STREAM-OLAP. Considering the TPC$D S$ benchmark and the fact table, we chose 8 dimensions of the TPC-DS benchmark that are directly connected to the "Store Sales" fact table. For each dimension, we chose 3 hierarchy levels.

Figure 6.22 shows the 8 chosen dimensions with their hierarchies. Note that due to the type of the available attributes in the last three dimensions, we did not find suitable and logical attributes to be used as the hierarchy levels of their dimensions. Hence, we assigned 1 or 2 hierarchy levels for these dimensions. Each column in the figure relates to one dimension. The first box for each dimension denotes the name of the dimension while the boxes below denote hierarchy levels from 1(the highest summarization level) to 3(the most detailed level). Dimensions Store, Item, Address, and Promotion are unordered dimensions, while other dimensions are ordered.

For queries, we generated a variety of OLAP query sets with different types, coverages, and sizes. We did not use the query templates in the TPC-DS data set since they are SQL 


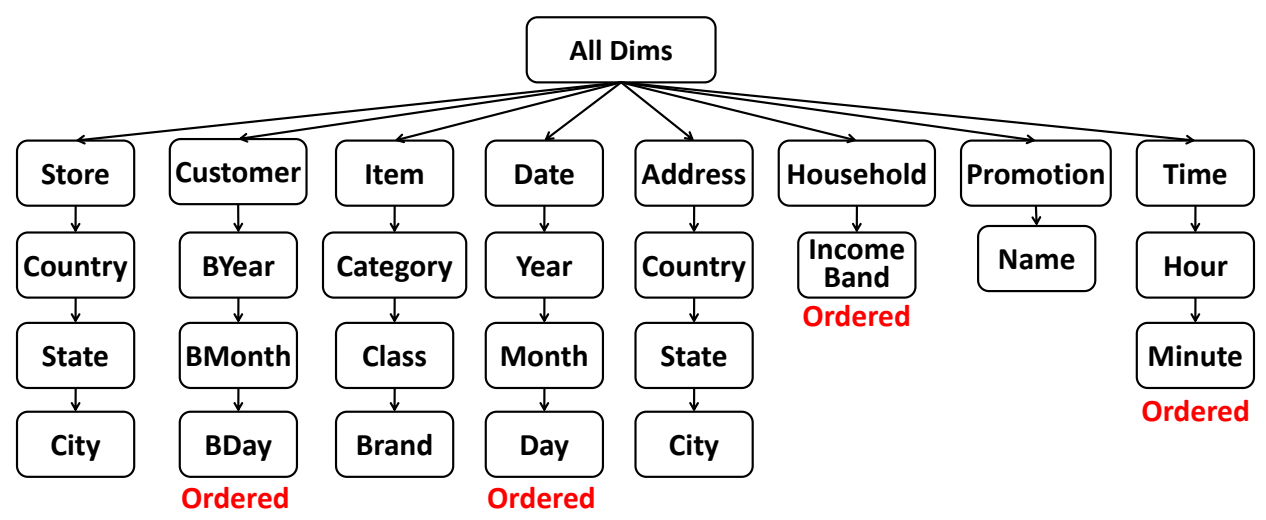

Figure 6.22: 8 dimensions of TPC-DS benchmark directly connected to the fact table "Store Sales". Boxes below each dimensions specifies hierarchy levels from 1 to 3 . Ordered dimensions are specified as "ordered" accordingly.

queries that tend to materialize a data cube rather than to provide direct OLAP queries. Our generated queries include different combinations of dimensions and hierarchy levels that can occur in real OLAP queries.

Test Types: We considered a variety of tests to evaluate the performance of the CR-OLAP in different situations as follows.

1. Evaluating the impact of the number of worker machines (speed-up test)

2. Evaluating the impact of system size (scale-up test)

3. Evaluating the impact of the number of dimensions

4. Evaluating the impact of individual dimensions and query coverages

5. Evaluating the impact of query patterns at different hierarchy levels

For the first three tests, we selected OLAP queries with $10 \%, 60 \%$ and $95 \%$ query coverages, which refer to the percentage of the entire range of values for each dimension that is covered by a given OLAP query. The selected OLAP queries therefore aggregate a small, medium and large portion of the database, resulting in very different workloads. Each coverage is measured per dimension (i.e. the percent of the number/range of covered values over the cardinality/range of that dimension). Thus, the coverage $X \%$ denotes $X \%$ 
for each dimension that results in a total database coverage of $\left(\frac{X}{100}\right)^{d}$. Considering the structure of the queries described in Section 6.3.4, there may be several query values in an unordered dimension to provide a high coverage. For example, our $95 \%$-coverage queries contain 48 query values in the Address dimension representing 48 states in the US. The following presents two query samples of our generated queries for the last two tests.

1. Store: Centerville, Midway, Fairview, Liberty, Customer: [1965-1-1, 1984-12-31], Item: Electrical, Date: [2013-1-1, 2013-1-3], Address: NY, CA, TN, AZ, TX, MN, OH, IA, NV, NJ, Household: [1000-3000], Promotion: Thanksgiving, Time: '*'

2. Store: CA, NY, Customer: '*', Item: ' ${ }^{*}$ ' Date: ['*'-2-'*', '*'-5-'*'], Address: '*', Household: '*', Promotion: '*', Time: '*'

As seen in the first example, attribute values in the Store dimension are all in the city level. Item values are in the category level, Address values in the state level, etc. We emphaize that PDCR-tree is able to process attribute values from different hierarchy levels of each dimension. The star value ('*') for a level in a dimension indicates all possible values of that level in the corresponding dimension. The second query example shows a specific feature of the PDCR-tree that is handling of queries that request for specific values/ranges in certain hierarchy levels of a dimension (slicing/dicing) while aggregating on all values of other levels in that dimension (rolling-up). The second query sample looks for "total sales in the stores located in California and New York during February-May of all years". Note that this feature is provided in a single query. Such a query will not be divided into multiple queries at the time of execution to cover e.g. different years that contribute to a '*' value. This feature shows the ability of the PDCR-tree to enable OLAP operations e.g. roll-up, drill-down, slice, and dice without materializing any data cube.

Comparison baseline: As outlined in Chapter 3, there is no comparison system for the $C R$ $O L A P$ that provides scalable cloud-based OLAP with full real-time capability and support for dimension hierarchies. To establish a comparison baseline for the CR-OLAP, we therefore designed and implemented a STREAM-OLAP method which partitions the database between multiple cloud processors based on one chosen dimension and uses parallel memory to cache streaming on the cloud processors to answer OLAP queries. More precisely, 
STREAM-OLAP builds a 1-dimensional index on one ordered dimension $d_{\text {stream }}$ and partitions the data into approx. $100 \times m$ arrays. The $1 \mathrm{D}$-index uses the Customer dimension to index the data into the 100 arrays. We chose the combination of three attributes from this dimension to build the index. The three attributes are birth year, birth month, and birth day of the customers. The arrays are randomly shuffled between the $m$ workers. The master processor maintains the 1-dimensional index. Each array represents a segment of the $d_{\text {stream }}$ dimension and is accessed via the 1-dimensional index. The arrays themselves are unsorted, and insert operations simply append the new item to the respective array. For query operations, the master determines via the 1-dimensional index which arrays are relevant. The workers then search those arrays via linear search, using memory to cache streaming.

The comparison between CR-OLAP (using PDCR-tree) and STREAM-OLAP (using a 1-dimensional index and memory to cache streaming) is designed to examine the tradeoff between a sophisticated data structure which needs fewer data accesses but is less cache efficient and a brute force method which accesses much more data but optimizes cache performance.

\subsubsection{Experimental Evaluation On Amazon EC2 Cloud}

Impact of the number of worker machines (speed-up test): We tested how the time of insert and query operations for the CR-OLAP and STREAM-OLAP changes for fixed database size $(N)$ as we increase the number of workers $(m)$. Using a variable number of workers $1 \leq m \leq 8$, we first inserted 40 million items (with $d=8$ dimensions) from the $T P C-D S$ benchmark into the CR-OLAP and STREAM-OLAP, and then we executed 1,000 (insert or query) operations on the CR-OLAP and STREAM-OLAP. Since workers are virtual processors in the Amazon EC2 Cloud, there is always some performance fluctuation because of the virtualization. We found that the total (or average) of 1,000 insert or query operations is a sufficiently stable measure. The results of our experiments are shown in Figures 6.23, 6.24, and 6.25.

Figure 6.23 shows the time for 1,000 insertions in the CR-OLAP (PDCR-tree) and 


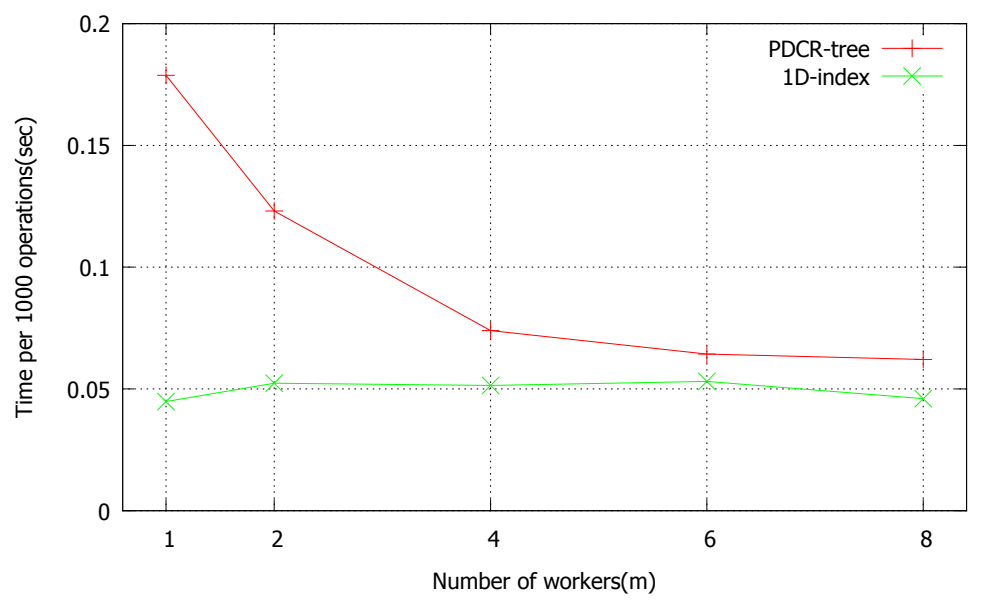

Figure 6.23: Time for 1000 insertions as a function of the number of workers. $(N=40 \mathrm{Mil}$, $d=8,1 \leq m \leq 8)$

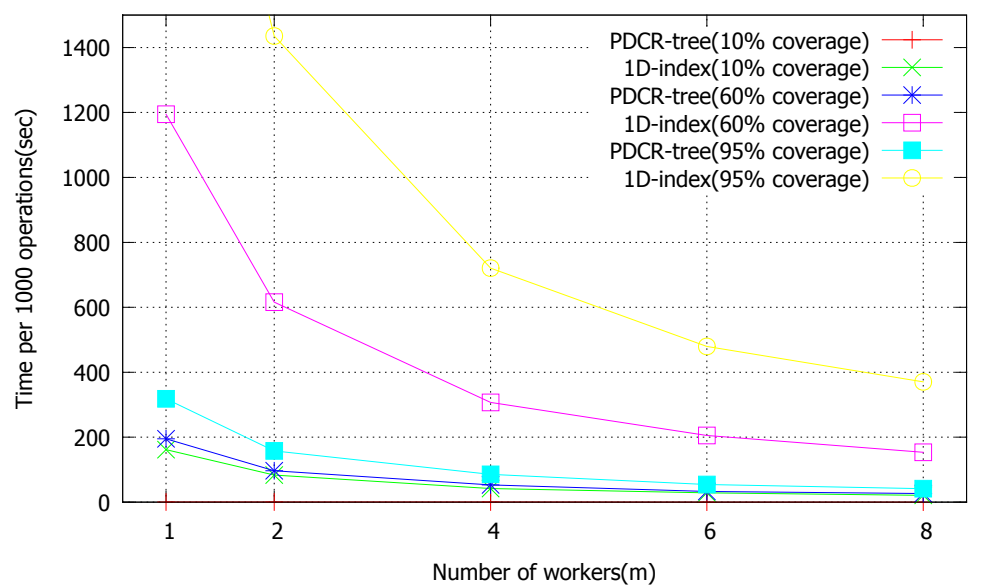

Figure 6.24: Time for 1000 queries as a function of the number of workers. $(N=40 \mathrm{Mil}$, $d=8,1 \leq m \leq 8)$

STREAM-OLAP (1D-index) as a function of the number of workers $(m)$. As expected, insertion times in the STREAM-OLAP are lower than in the CR-OLAP because the STREAMOLAP simply appends the new item at the end of array while the CR-OLAP has to perform tree insertions with tree traversal, possible directory node splits and other overheads. However, the STREAM-OLAP shows no speedup with increasing the number of workers (because only one worker performs the array append operation) whereas the CR-OLAP shows a significant speedup (because the distributed PDCR-tree makes use of the multiple 


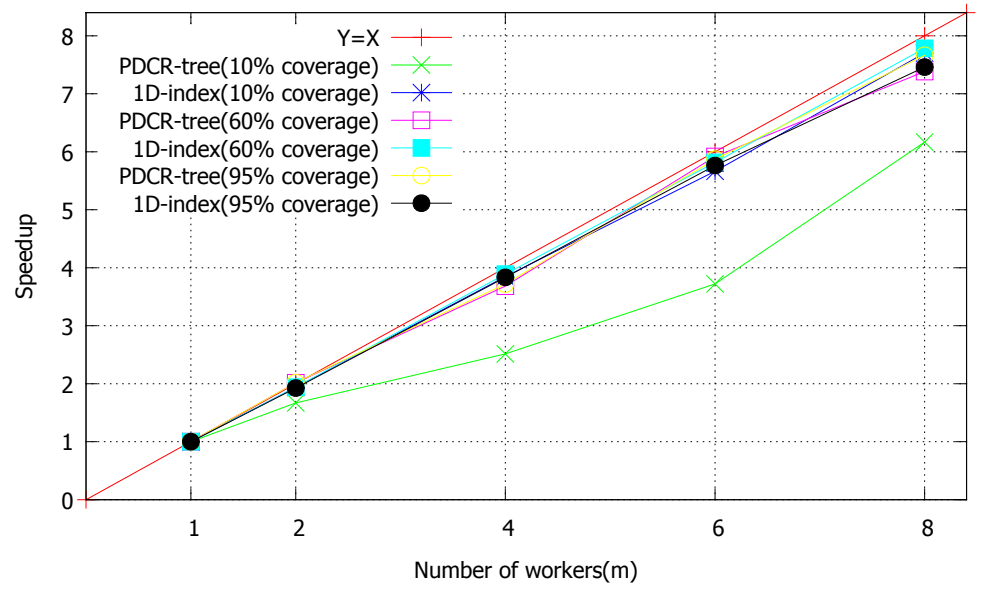

Figure 6.25: Speedup for 1000 queries as a function of the number of workers. $(\mathrm{N}=40 \mathrm{Mil}$, $d=8,1 \leq m \leq 8)$

workers). This is despite the fact that the hat of the PDCR-tree is operating sequentially on 1 master machine. It is important to note that insertion times are not visible to the users because they do not create any user response. What is important to the user are the response times for OLAP queries. Figure 6.24 shows the time for 1,000 OLAP queries in the $C R$ OLAP and STREAM-OLAP as a function of the number of workers $(m)$. Figure 6.25 shows the speedup measured for the same data. We observe in Figure 6.24 that the CR-OLAP significantly outperforms the STREAM-OLAP with respect to query time. The difference in performance is particularly pronounced for queries with small(10\%) or large(95\%) coverages. For the former, the tree data structure shows close to logarithmic performance and for the latter, the tree can compose the result by adding the aggregate values stored at a few roots of large subtrees near the root of the distributed PDCR-tree. The worst case scenario for the CR-OLAP are queries with medium coverage around $60 \%$ where query conditions are true for the majority of directory nodes. This enforces the threads to check a large portion of the tree to find every small part of the tree that meets query conditions. However, even in this worst case scenario, the CR-OLAP outperforms the STREAM-OLAP. Figure 6.25 indicates that both systems show a close to linear speedup with increasing the number of workers, however for the CR-OLAP that speedup occurs for much smaller absolute query times. 
Impact of system size (scale-up test): In an elastic cloud environment, the CR-OLAP and STREAM-OLAP increase the number of workers $(m)$ as the database size $(N)$ increases. This test shows the performance of the CR-OLAP and STREAM-OLAP as the system scales up. We scaled up the system size from $N=10 M, m=1$ to $N=80 M, m=8$. The impact on the performance of insert and query operations is shown in Figures 6.26 and 6.27, respectively.

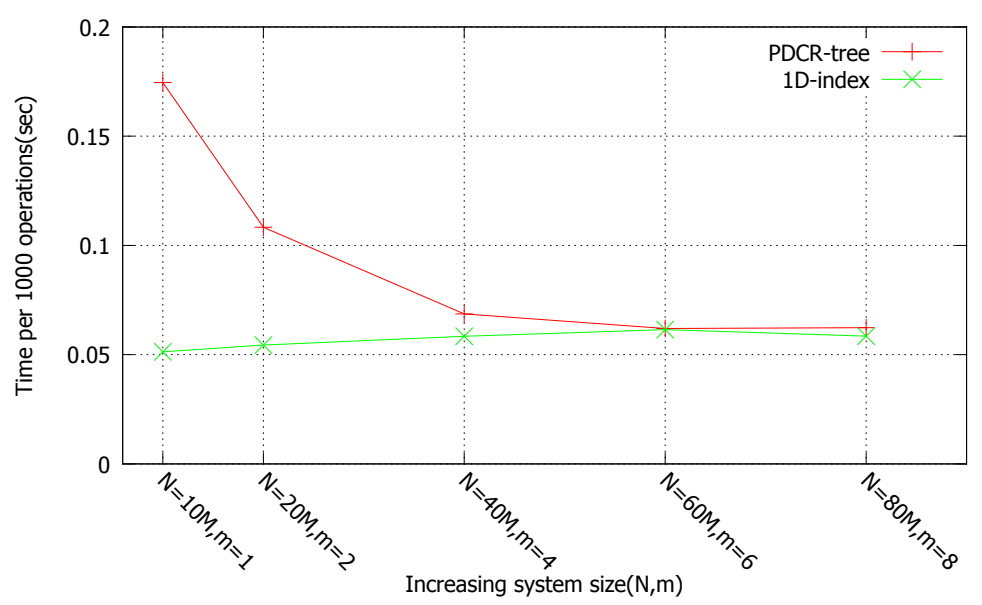

Figure 6.26: Time for 1000 insertions as a function of system size: $N \& m$ combined. $(10 \mathrm{Mil} \leq N \leq 80 \mathrm{Mil}, d=8,1 \leq m \leq 8)$

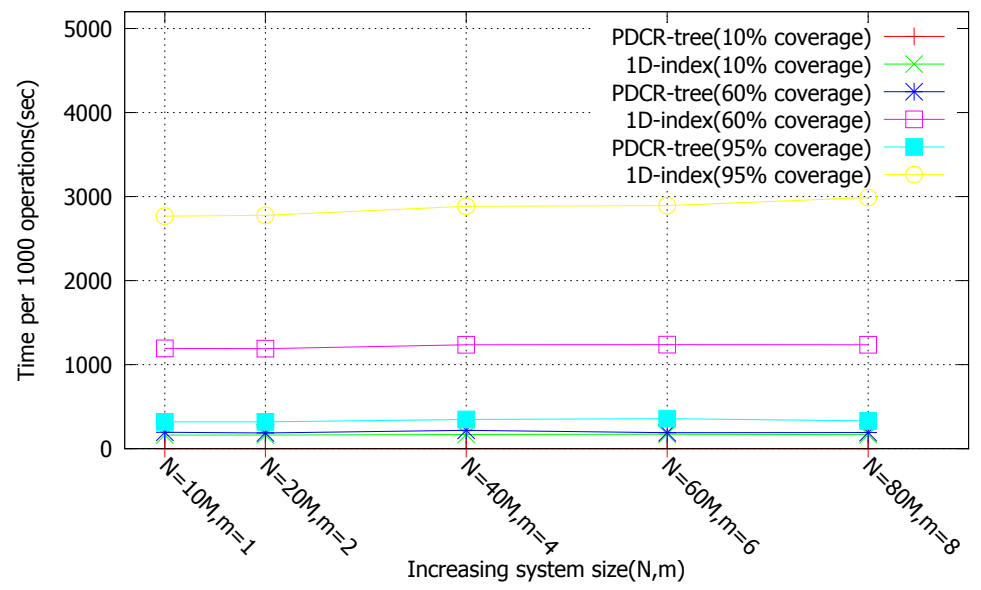

Figure 6.27: Time for 1000 queries as a function of system size: $N \& m$ combined. $(10 \mathrm{Mil} \leq N \leq 80 \mathrm{Mil}, d=8,1 \leq m \leq 8)$ 
With growing system size, the time for insert operations in the CR-OLAP (PDCR-tree) approaches the time for the STREAM-OLAP (1D-index). More importantly however, the time for query operations in the CR-OLAP again outperforms the time for the STREAM$O L A P$ by a significant margin, as shown in Figure 6.27. Also, it is very interesting that for both systems, the query performance remains essentially unchanged with increasing database size and the number of workers. This is obvious for the STREAM-OLAP where the size of arrays to be searched simply remains constant but it is an important observation for the CR-OLAP. Figure 6.27 indicates that the overhead incurred by the CR-OLAP's load balancing mechanism (which grows with increasing $m$ ) is balanced out by the performance gained through more parallelism. The $C R$-OLAP appears to scale up without affecting the performance of individual queries. It performed an 8 -fold increase in database size and the number of processors, including an 8 -fold increase in the average amount of data aggregated by each OLAP query, without noticeable performance impact for the user.

Impact of the number of dimensions: It is well known that tree based search methods can become problematic when the number of dimensions in the database increases. This test evaluates the impact of dimensionality on the performance. We performed this test for 5 different number of dimensions in the range $4 \leq d \leq 8$. For each number of dimension, insertions were followed by three sets of regular queries for three coverages small(10\%), medium(60\%), and large(95\%). In Figures 6.28 and 6.29 we show the impact of increasing $d$ on the performance of insert and query operations in the CR-OLAP (PDCR-tree) and STREAM-OLAP (1D-index) for fixed database size $N=40$ million and $m=8$ workers.

Figure 6.28 shows some increase in insert time for the CR-OLAP because the PDCRtree insertion inherits from the $P D C$-tree a directory node split operation with an optimization phase that is highly sensitive to the number of dimensions. However, the result of the tree optimization is improved query performance in higher dimensions. As shown in Figure 6.29 , the more important time for OLAP query operations grows only slowly as the number of dimensions increases. This is obvious for the array search in the STREAM-OLAP but for the tree search in the CR-OLAP, this is an important observation.

Impact of individual dimensions and query coverages: The fourth and fifth tests focus 


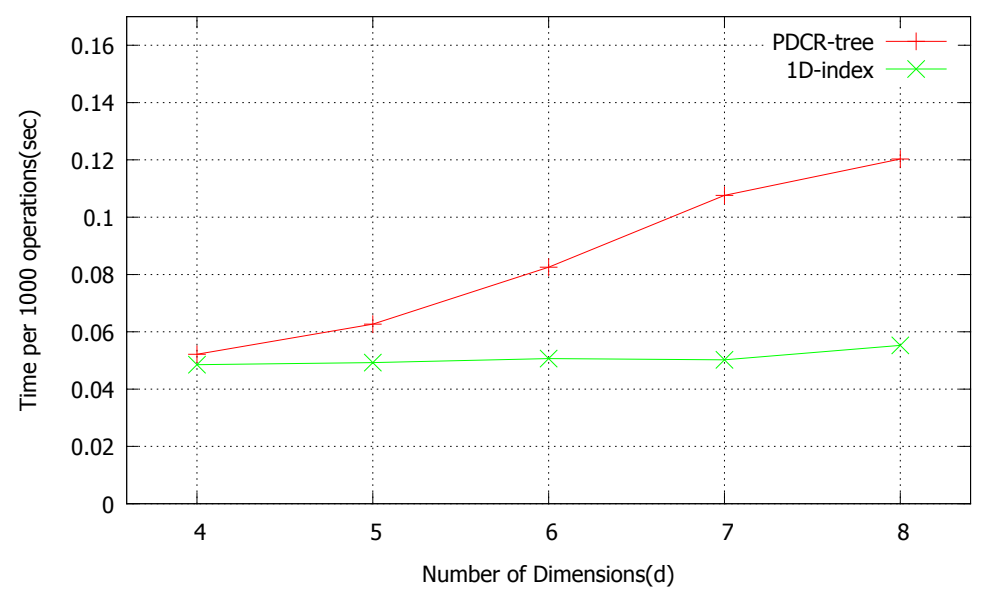

Figure 6.28: Time for 1000 insertions as a function of the number of dimensions. $(N=$ $40 \mathrm{Mil}, 4 \leq d \leq 8, m=8)$

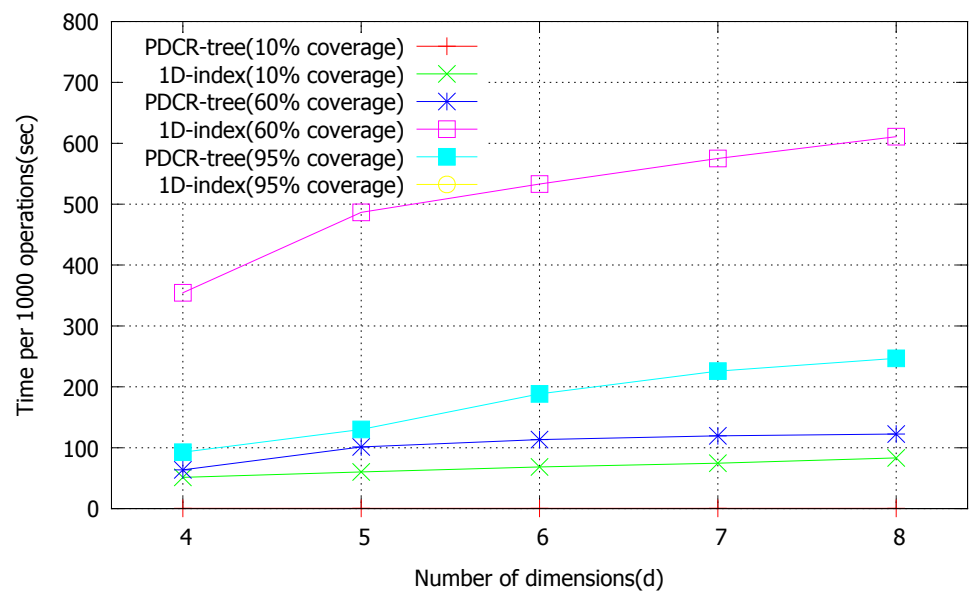

Figure 6.29: Time for for 1000 queries as a function of the number of dimensions. The values for "1D-index 95\% coverage" are 828.6, 1166.4, 1238.5, 1419.7 and 1457.8, respectively. $(N=40 \mathrm{Mil}, 4 \leq d \leq 8, m=8)$

on the different types of OLAP queries. We generated 9 sets of queries each with different coverages from $10 \%$ to $90 \%$ and from $91 \%$ to $99 \%$. In each set except for the ninth set, we applied a “*” value for one of the 8 dimensions. There is no "**" value in the ninth set. In this test, we aim to show how dimensions with different coverages influence the performance of the tree. In addition, this test includes an interesting case where the "*" value is applied to the "Customer" dimension. The "*" value for the "Customer" dimension 
creates a bottleneck for the STREAM-OLAP (1D-Index) (and possibly any 1-dimensional indexing structure) since it indexes arrays using this dimension. To answer such queries, the STREAM-OLAP (1D-Index) has to search all arrays leading to a serious performance issue. Query examples for this case are common in real-life applications (e.g. "the total sales for all customers born any time...”). Figures 6.30 and 6.31 show the PDCR-tree runtime to perform $q=1000$ queries for each of the nine sets for coverages $10 \%-90 \%$ and $91 \%-99 \%$, respectively. Figures 6.32 and 6.33 depict the ratio of 1D-index runtime over the distributed PDCR-tree runtime for the same sets of queries on the same data.

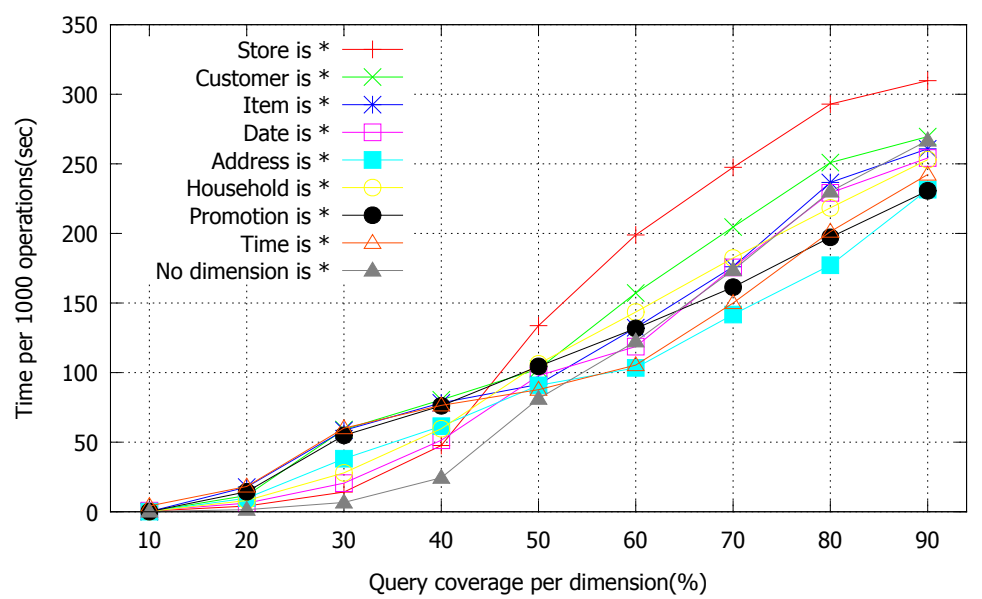

Figure 6.30: Time for 1000 queries (PDCR tree) as a function of query coverages: $10 \%-$ $90 \%$. Impact of value “*” for different dimensions. $(N=40 \mathrm{Mil}, m=8, d=8)$

A higher ratio indicates a faster response time for the CR-OLAP compared to the STREAM-OLAP. As the curves in the figures illustrate, the tree outperforms the $1 \mathrm{D}$-index with a wide margin for all sets of queries. Figures 6.30 and 6.31 show that the "*" values in the queries do not have a significant impact on the $C R-O L A P$. As discussed earlier, the $C R-O L A P$ is most efficient for small and large query coverages, with maximum query time somewhere in the mid range. (In this case, the maximum point is shifted away from the typical $60 \%$ because of the “*” values.)

Figures 6.32 and 6.33 show the performance of the STREAM-OLAP as compared to the $C R-O L A P$ (ratio of query times). It shows that the CR-OLAP consistently outperforms the STREAM-OLAP by a factor between 5 and 20. An interesting observation is that by increasing the query coverage from $10 \%\left(10^{-8} \%\right.$ of the whole database) to around $91 \%(50 \%$ 


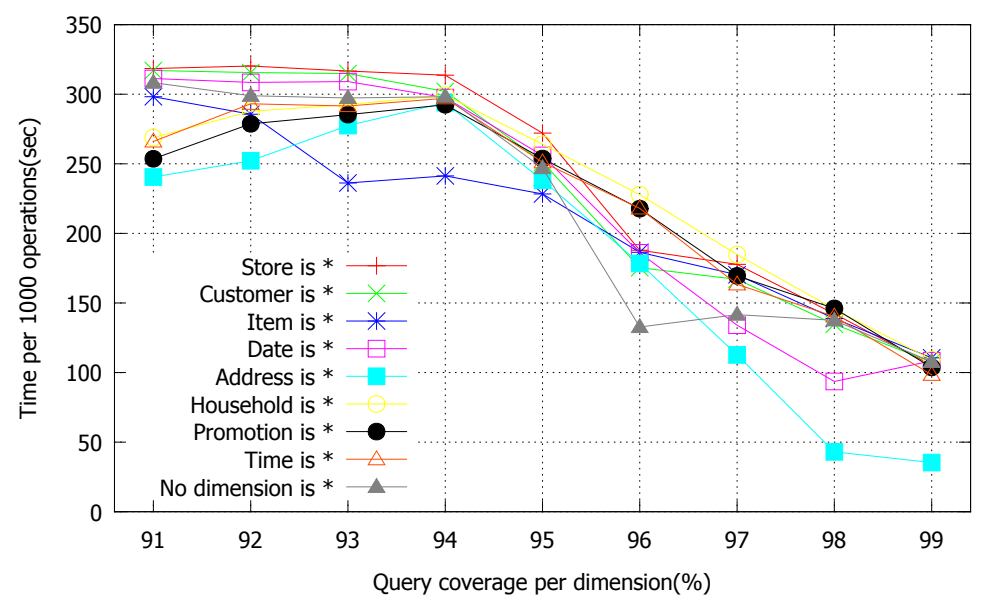

Figure 6.31: Time for 1000 queries (PDCR tree) as a function of query coverages: $91 \%-$ $99 \%$. Impact of value “*” for different dimensions. $(N=40 \mathrm{Mil}, m=8, d=8)$

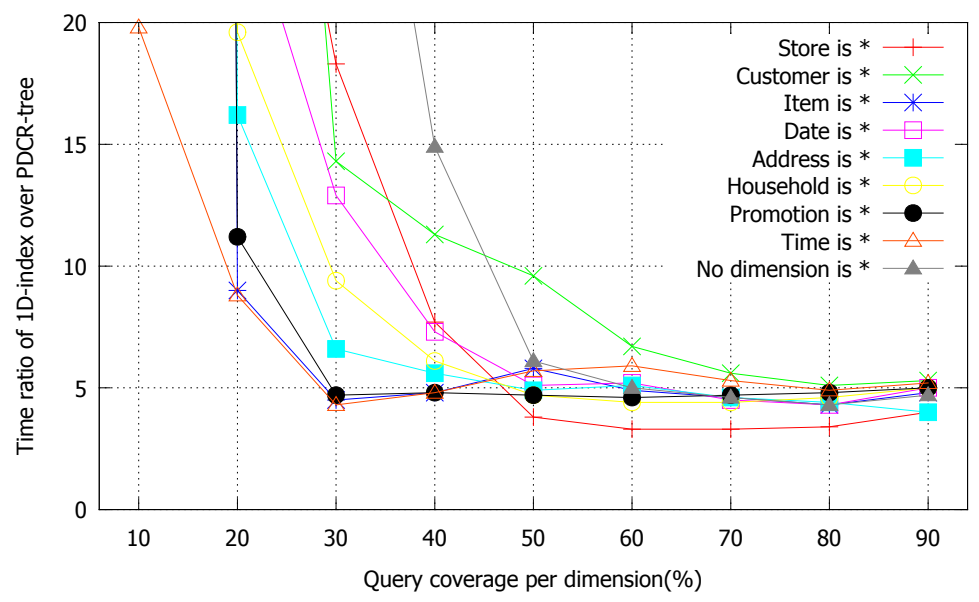

Figure 6.32: Time comparison for 1000 queries (Ratio: 1D-index / PDCR tree) for query coverages $10 \%-90 \%$. Impact of value “*” for different dimensions. $(N=40 \mathrm{Mil}, m=8$, $d=8)$

of the whole database) the runtime for the $C R-O L A P$ increases. This is expected since for larger coverages, the $C R-O L A P$ needs to search and retrieve a larger portion of the database. However, this effect stops at the total coverage of $50 \%$ of the database.

After this point by increasing the query coverage, the $C R-O L A P$ gains the advantage to not search lower parts of the distributed PDCR-tree since they are fully covered in the queries with a high coverage. In such cases, the tree only tests the enclosure of the directory 


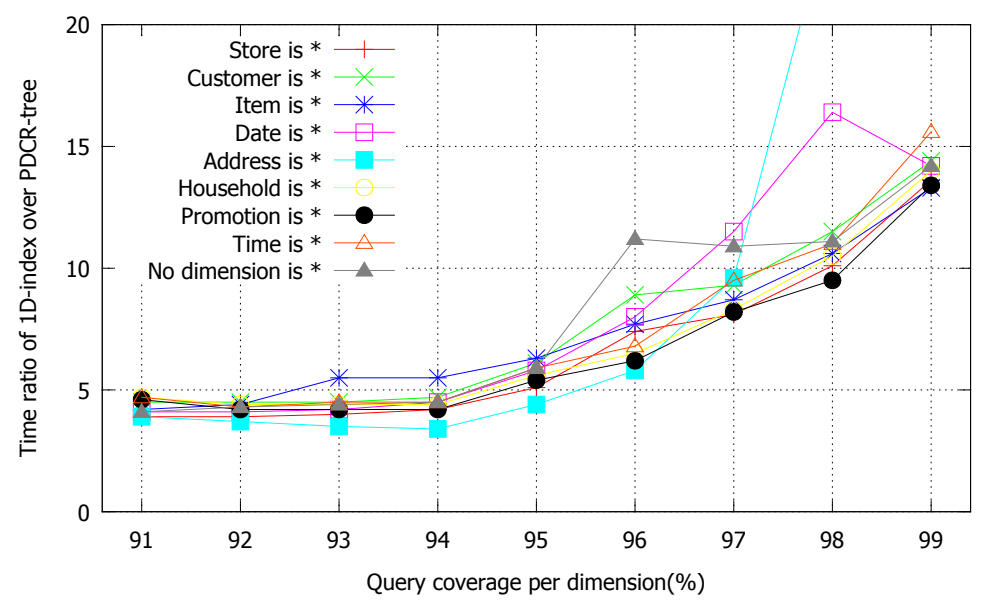

Figure 6.33: Time comparison for 1000 queries (Ratio: 1D-index / PDCR tree) for query coverages $91 \%-99 \%$. Impact of value “*” for different dimensions. $(N=40 \mathrm{Mil}, m=8$, $d=8)$

nodes that are usually the parents of large parts of the tree, and may add them to the result set without further processing of their children. This effect is clearly observed in the curves of Figure 6.31. By increasing query coverage from $91 \%(50 \%$ of the whole data base) to $99 \%(92 \%$ of the whole data base), the query time decreases for all query sets. The 1Dindex does not have this advantage and constantly degrades in performance by increasing query coverage.

Impact of query patterns at different hierarchy levels: An important feature of the $C R$ $O L A P$ is its capability to answer OLAP queries such as "total sales in the stores located in California and New York during February-May of all years" which act at the different levels of multiple dimension hierarchies. Answering such queries using a data cube approach would require expensive operations such as roll-up, drill-down, slice, and dice. However, the distributed PDCR-tree skips these expensive tasks by using its hierarchical and multidimensional tree structure. This test evaluates the performance of the CR-OLAP to answer such queries. For fixed database size $N=40 \mathrm{Mil}$, number of workers $m=8$ and $d=8$ dimensions, we test for the Date dimension the impact of "*” for different hierarchy levels. We created 7 combinations of the “*” values, and set the values for hierarchy levels Year, 
Month, and Day as follows: *-*_*, year-*-*, year-month-*, year-month-day, *-month-*, *month-day, and $*_{-} *_{-}$day. We then selected for each combination queries with the coverages $10 \%, 60 \%$, and $95 \%$.

Figure 6.34 shows a query time comparison between the CR-OLAP (PDCR-tree) and STREAM-OLAP (1D-index) for selected query patterns. Considering the ID structure, the

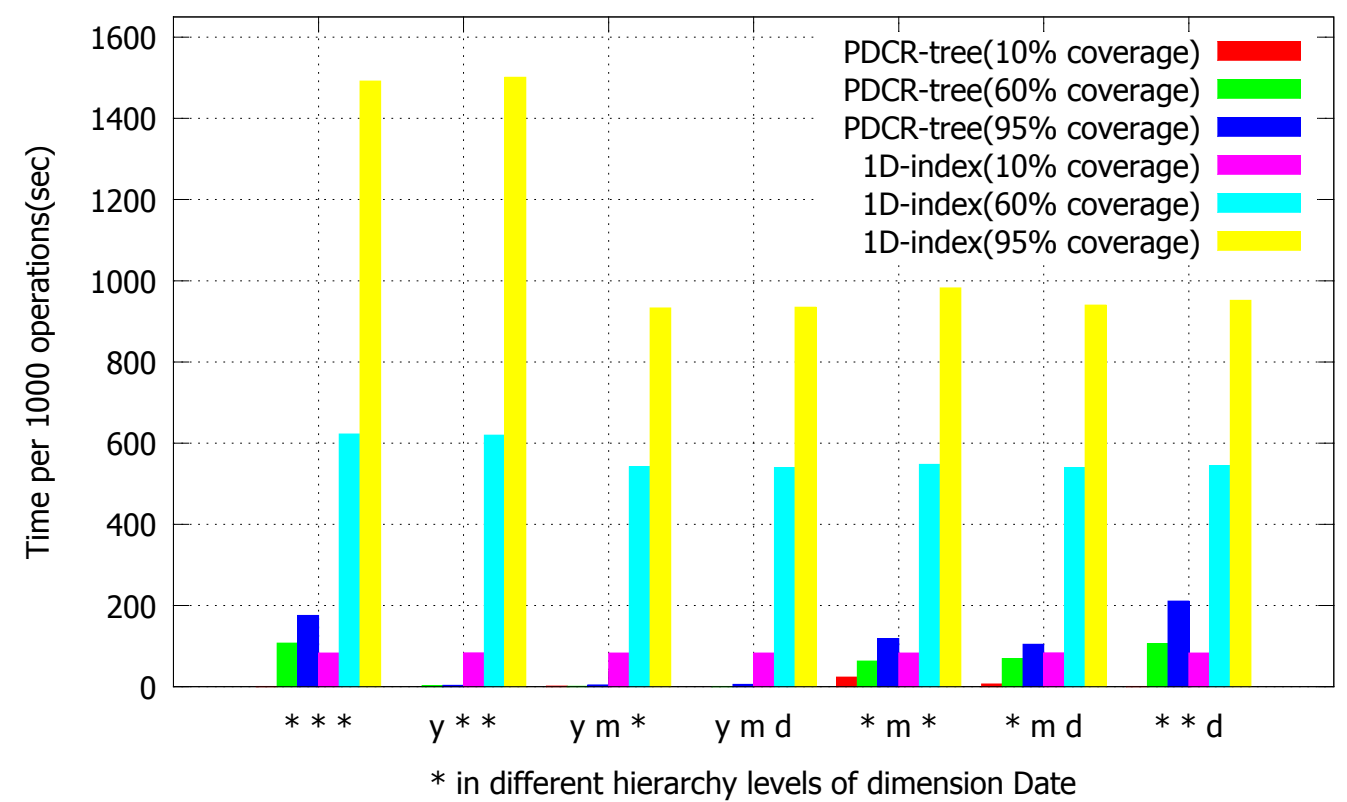

Figure 6.34: Query time comparison for selected query patterns for dimension Date. Impact of value "*”" for different hierarchy levels of dimension Date. $(N=40 \mathrm{Mil}, m=8$, $d=8)$.

PDCR-tree firstly indexes the data using the first hierarchy level of each dimension. It uses the second and third levels only when a sub-space of the data is fully inside the hierarchy values of the first level for its corresponding dimension. Therefore, it is expected for a query such as "total sales in a specific month in all years..." to not directly benefit from the indexing. In fact for such cases, the PDCR-tree is expected to approach a linear search as the query requests specific values for lower hierarchy levels. However it is interesting to evaluate to which degree the tree degrades to answer such queries. The three combinations *-month-*, *-month-day, and *-*-day among the 7 combinations above create such queries.

As seen in the figure, the distributed PDCR-tree outperforms the 1D-index for all cases. In fact, the PDCR-tree benefits from a second and third level indexing in lower areas of the 
tree and does not degrade to a naive linear search.

In this chapter, we measured the performance of the PDC-tree, PDCR-tree, and CR$O L A P$ based on the response time of the implemented systems. In fact, the main goal for these methods is to minimize the response time of the system to answer received requests. Based on the experimental results in this chapter, we observe that there is a reverse relationship between the response time of the methods with the number of cores in each machine $(p)$ and number of utilized workers in the $C R-O L A P(m)$. However, this relationship is direct between the response time and the total number of data records in the system $(N)$, and the number of dimensions in the data warehouse $(d)$. Hence, we may provide the following performance model for the PDC-tree and PDCR-tree methods.

$$
\text { ResponseTime } \propto \frac{N \times d}{p \times m}
$$

\subsection{Summary}

In this chapter, we presented the main contribution of this thesis. We presented the $P D C$ tree method, PDCR-tree and CR-OLAP to build real-time OLAP systems using multi-core and cloud platforms. The OLAP servers provided real-time response to every OLAP query regardless of its size and coverage. Our OLAP methods answered the largest OLAP query with 85 different values in 8 dimensions covering 95\% of each dimension, in a data warehouse with 80 million records distributed over 8 machines in 0.3 seconds. According to our definition of the real-time response for real-time OLAP systems, the offered methods successfully provided a real-time response for every OLAP query using the most recent updated data in the data warehouse. 


\section{Chapter 7}

\section{Conclusion}

\subsection{Introduction}

In this chapter we summarize the contributions and results achieved in the previous chapters. This thesis provided discussions and software methods in the three following subjects:

1. Parallel Sorting on Many-core GPUs

2. Parallel Building of Static Data Cubes on Multi-core Processors

\section{Real-time OLAP on Multi-core and Cloud Platforms}

For each subject, the motivations and problems were declared in Chapter 1. Next, the background and related work of the subjects were reviewed in Chapters 2 and 3, respectively. We then presented a detailed discussion of the new offered methods as our contribution to the problems. For each subject, the offered methods were evaluated in parallel hardware platforms and were compared to other related methods where applicable. In the next section, we provide a summary of the achieved results for the proposed methods in this thesis.

\subsection{Summary of Results}

In Chapter 4, we offered a parallel sorting method for many-core GPUs called GPU Bucket Sort. Our experimental evaluation indicated that the GPU Bucket Sort is considerably faster than Thrust Merge [98], the best comparison-based sorting algorithm for GPUs at the time of development, and it is exactly as fast as randomized sample sort for GPUs (GPU Sample Sort) [81] when the input data sets are uniformly distributed, which is a best case scenario for randomized sample sort. However, as observed in [81], the performance of the randomized GPU Sample Sort fluctuates with the input data distribution whereas the GPU 
Bucket Sort does not show such fluctuations. The GPU Bucket Sort showed a fixed sorting rate (number of sorted data items per time unit) for the entire range of data sizes tested (up to $n=512 M$ data items), whereas it is shown in [81] that the sorting rate for randomized GPU Sample Sort fluctuates and often starts to decrease for larger values of $n$.

In Chapter 5, we presented a method for parallel building of static data cubes using multi-core processors with multiple parallel disks. Our experimental results for a Sandy Bridge multi-core processor with 4 parallel disks indicated that the $M C M D-C U B E$ achieves $50 \%$ of the theoretically optimal linear speedup by increasing the number of cores and disks. The MCMD-CUBE method applied a parallel external sorting algorithm called $M C M D-S O R T$ which was also developed by the author. We compared the MCMD-SORT method with a state-of-the-art sorting method from the STXXL library for multi-core processors. A comparison of results showed that the MCMD-SORT outperforms the sorting method available in the STXXL library.

In Chapter 6, we presented the main contribution of this thesis in providing Real-time $O L A P$. We proposed new methods and algorithms for building Real-time OLAP systems on multi-core and cloud platforms. Two multi-dimensional trees called PDC-tree and PDCRtree were developed to provide a parallel data structure for Real-time OLAP. We then developed new parallel algorithms to support parallel OLAP operations in the trees. In addition, we developed a framework called CR-OLAP as the structure of our cloud solution in cloud architectures. The CR-OLAP utilizes the PDCR-tree to build Real-time OLAP servers in cloud environments.

According to the achieved results, the PDC-tree answers every OLAP query in realtime in less than 1 second while real-time updates are simultaneously occurring in the tree. In a test case for a database with 10 million data records, the $P D C$-tree achieved a speedup of nearly 20 on the Intel Xeon Westmere EX processor with 20 processor cores. The results also confirmed that the $P D C$-tree answers OLAP queries faster than the MYSQL in most cases for 4 query coverages out of 5 cases.

For cloud-based Real-time OLAP, the CR-OLAP established a real-time response to every OLAP query regardless of its size and coverage. The distributed PDCR-tree was found to be particularly efficient for complex OLAP queries that need to aggregate a large portion of the data warehouse, such as "Report the total sales in all stores located in California and 
New York during the months February-May of all years". In the worst case, the CR-OLAP answered the largest OLAP query with 85 different values in 8 dimensions covering $95 \%$ of each dimension, in a data warehouse with 80 million records distributed over 8 machines in 0.3 seconds. The tests also indicated that the CR-OLAP scales well with increasing the number of workers. For example, for a fixed data warehouse size (10 million data items), when the number of workers is increased from 1 to 8 , the average query throughput and latency improves by a factor 7.5 which is considered nearly a linear speedup.

According to our definition of the real-time response for Real-time OLAP systems, the offered methods successfully provided a real-time response for every OLAP query using the most recent updated data in the data warehouse. The provided methods in this thesis have shown their potential to build Real-time OLAP servers for large data warehouses in multi-core and cloud environments.

\subsection{Observations}

In this section we outline findings, outcomes, and what we learned in the three subjects of this thesis. The findings are observations and technical knowledge that we learned by development and evaluation of the methods and algorithms for the parallel architectures in this thesis.

During the development and evaluation of the GPU Bucket Sort method, we found that recent parallel GPUs are efficient parallel processors that have a high potential to compete with multi-core CPUs. As many algorithms including sorting methods are shown to be faster on GPUs, computer applications may now rely partly on the high processing power of GPUs and divide their work load between CPU and GPU. GPU is now a firm computing resource available on many servers. Organizations may use servers that provide a combination of the two computing resources in every node.

In addition, GPU Bucket Sort was shown to effectively use the parallel cores on Nvidia GPUs as we upgraded the GPUs and increased the number of available cores. We observed that by increasing the number of threads, GPU Bucket Sort utilizes more cores and sorts faster.

During the development and evaluation of the $M C M D-C U B E$, we learned that sorting is a key step in building data cubes. Since data cube is composed of many cuboids where each 
cuboid requires a significant sorting step, it is crucially important to use an efficient sorting method. However, we observed that the performance of the traditional data cube building methods are degrading even when utilizing recent parallel platforms. We found that materializing data cubes is an inefficient way to utilize parallel hardware resources. This observation brought us to the study that we outlined in Chapter 6 of this thesis. We found that for the present and future scales of enterprise data, a traditional method such as data cube suffers from many drawbacks such as huge memory and CPU requirements. We concluded that a dynamic multi-dimensional indexing structure has the basics and primitives to enable a Real-time OLAP system. This is what we introduced as PDC-tree, PDCR-tree, and $C R$-OLAP in Chapter 6.

We found that building/computing aggregations as group-bys/cuboids in a data cube method ahead of receiving requests, is not an efficient method for Real-time OLAP. With today's large enterprise data, by the time a data cube and its cuboids are computed, the data in the data cube is out dated. By introducing PDC-tree and PDCR-tree methods, we suggested that rather than pre-computing the data cube in order to have ready answers for OLAP queries, we can index the data in a proper way so that data updates, queries, and other database operations can be completed within a real-time deadline. Our results in Chapter 6 assured that such an approach is feasible using today's parallel and distributed hardware architectures.

During the evaluation of the $P D C$-tree method, we learned that it is important to properly handle ordered and unordered dimensions. This led us to applying a non-trivial enhancement on the PDC-tree and introducing the PDCR-tree. For the PDCR-tree method and during the development of the CR-OLAP framework, we observed that the Amazon $E C 2$ Cloud does not provide sufficient transparency for the rack layers that are in between the instances. In our experiments, since the size of the CR-OLAP was not large (i.e. at most 8 workers), we have not observed instances to be distributed on different racks. However, with a larger system, instances may be chosen from different racks resulting in different network latencies. This may be misleading in particular when real-time systems are tested using Amazon EC2 Cloud.

Finally, we observed that a multi-dimensional data index can be parallelized with a fine granularity down to the node level to provide a highly parallel data structure traversable by 
parallel database transactions. We also showed that a parallel multi-dimensional index that indexes data on all dimensions outperforms a 1-dimensional index that benefits from data caching.

\subsection{Future Work}

As the next steps in the development of Real-time OLAP systems, we offer the following future ideas and plans in two folds.

\subsubsection{Improvement on fault-tolerance}

Since the current design of the CR-OLAP comes with 1 master machine in the $m+1$ cloud nodes, one can argue that the master becomes a bottleneck at some point as the system scales up. Increasing the total data size and the number of workers arises the workload of the master machine. We have shown in our tests that currently, the workload of the master is slight compared to those of the worker machines. However, by growing data size up to the scale of Big Data (i.e. a few hundred terabytes and petabytes), the master can become a bottleneck. In addition, the master is a single point of failure. Our current design does not include a fault tolerance strategy to handle the failure of the master machine. As future work, it is an opportunity to develop a cloud framework that includes multiple master machines to effectively handle a master failure, and replace the failed master with a new master node. Figure 7.1 shows an example of a multiple-master scheme for the CR-OLAP.

As shown in the figure, in such a scheme the main master (denoted by Master 1 in the figure) receives the input stream of insertions and queries from clients. It then wraps the received requests as transactions. As soon as the main master assigns a thread to a transaction to start the hat traversal, it also sends a copy of the transaction to other masters in order to update them. Note that since query transactions do not cause any update or change in the hat, we do not need to send a copy of them to the other masters. The main master sends copies of only insertion transactions to the other masters. This reduces the additional workload caused by the sending process. The other masters receive the sent transactions, and apply them accordingly to their hats. However, after they completed the hat traversal step, they will not dispatch the insertion transactions to the workers. 


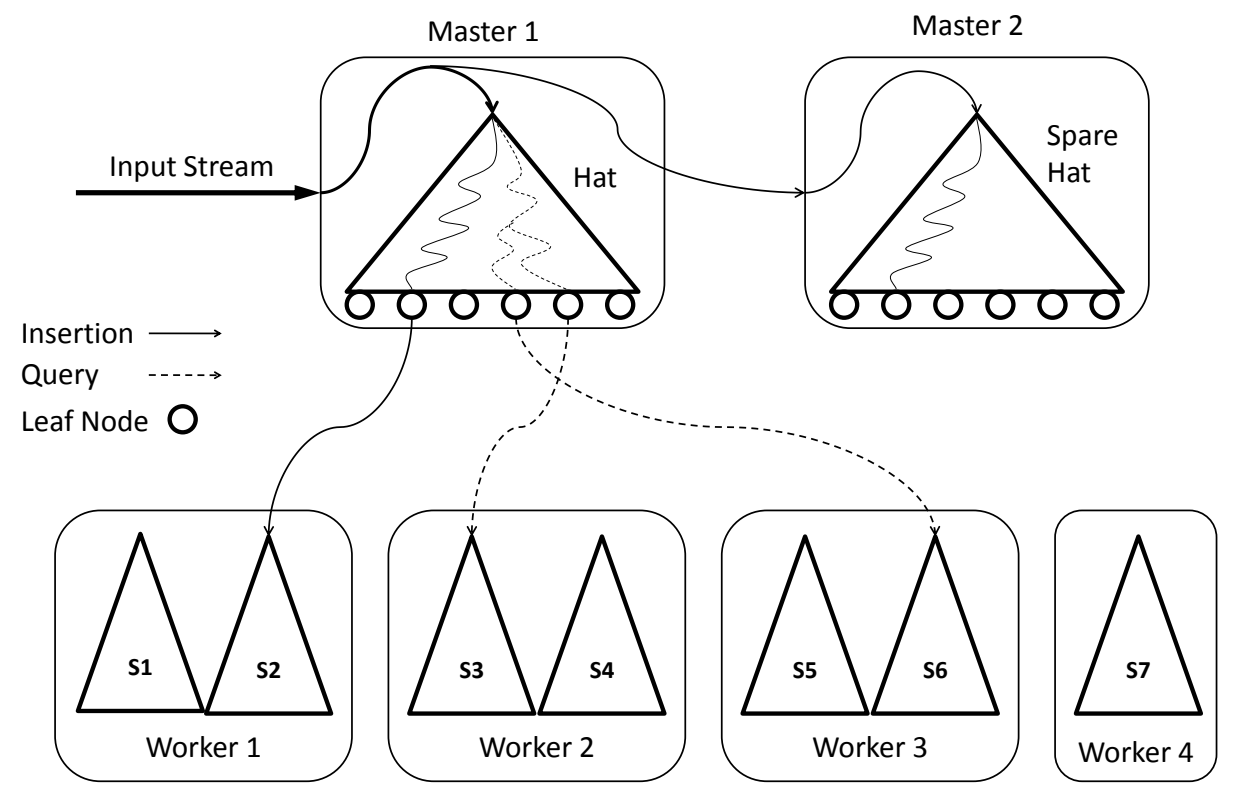

Figure 7.1: A multiple-master scheme for $C R$-OLAP. The first master at left receives the input stream from clients and dispatches a copy of only update operations (e.g. insertion) to the second master at right.

By applying the described method, the CR-OLAP creates one copy of the hat in each of the other master machines while assuring the smooth and normal flow of transactions in the main master and workers. The hat copies in the other masters get instantaneously updated upon arriving every new insertion transaction, and hence provide up-to-date copies of the $h a t$ that can replace it any time. Other masters in cooperation with the main master may run a heart beat process to consistently check the status of the main master. In case of a main master failure, the other master machines detect it, and may replace the main master with a new master.

\subsubsection{Scaling up to Big Data}

Our experimental results for Real-time OLAP scaled up to 80 million data records. However, we were not able to evaluate the provided methods for larger data warehouses due to the restrictions on research budgets. An argument would be that today's Big Data systems are supposed to handle petabytes of data. Indeed, our second suggestion as future work is to scale the developed framework for data sizes up to the edges of Big Data. Current Big 
Data platforms such as Hadoop [3] and Hive [108] are designed to handle tera and petascale databases. It is true that these platforms have not yet provided Real-time OLAP in Big Data contexts; however the demand still remains unsatisfied. As future work, we suggest to extend the experimental evaluations for the CR-OLAP and distributed PDCR-tree for data warehouses in the scale of Big Data, and verify a real-time and up-to-date response for every OLAP query in such scales. However in such scales, applying a more fault-tolerant scheme such as the scheme suggested in Section 7.4.1 seems to be inevitable.

\subsection{Publications}

The contributions and achieved results in this thesis have been published in the following conferences and journals:

1. Parallel Sorting on Many-core GPU Platforms

- "Deterministic sample sort for GPUs" published in the journal of parallel processing letters [50]

2. Parallel Building of Static Data Cubes on Multi-core Platforms

- "Parallel data cubes on multi-core processors with multiple disks" published in the proceedings of CASCON 2011 conference [49]

- "Parallel construction of data cubes on multi-core multi-disk platforms" published in the journal of parallel processing letters [51]

3. Real-time OLAP on Multi-core and Cloud Platforms

- "Parallel real-time OLAP on multi-core processors" published in the proceedings of CCGrid 2012 conference [41] 
- “A Distributed Tree Data Structure For Real-Time OLAP On Cloud Architectures" published in the proceedings of IEEE BigData 2013 conference [48]

- "Parallel Real-Time OLAP on Multi-Core Processors" to appear in the International Journal of Data Warehousing and Mining (IJDWM)

- "Scalable Real-Time OLAP On Cloud Architectures" submitted to the Journal of Parallel and Distributed Computing, Special Issue of Scalable Systems for Big Data Management and Analytics

\subsection{Achievements}

The methods and results presented in this thesis, won/achieved the following awards and honors as listed below.

\section{Patent Application}

The PDC-tree method presented in Chapter 6, was submitted as a patent application sponsored by IBM Centre for Advanced Studies (CAS) Canada in 2012. The application is in process. The patent application is titled as "Method and Apparatus for Parallel Computing of DC-Trees".

\section{IBM Canada "Innovation Impact of the Year 2012" Award}

The PDC-tree method also won the innovation impact of the year 2012 award. The award is presented by IBM CAS for "Advancement of Science that Leads to Significant Business Impact for IBM". Please refer to the following web page for details: https://www-927.ibm.com/ibm/cas/cascon/2012/awards.jsp

\section{Best Student Paper}

The MCMD-CUBE method presented in Chapter 5, published in [49] won the best student paper award in CASCON 2011 conference, Toronto. 


\section{Appendix A}

\section{Correctness Proofs for Splitting Subtrees in PDCR-tree}

In this appendix, we provide definitions, lemmas, and theorems to prove correctness of the subtree split operation inside a PDCR-tree. More precisely, we prove that by inserting new data nodes and creating new directory nodes into a PDCR-tree, a subtree can safely remove its horizontal links from/to neighbor subtrees and become independent. As we show in the following, independent subtrees are allowed to migrate between cloud machines providing a capability to distribute and balance them over cloud nodes.

\section{Definition 1: Branch}

Assume we have a set $S$ of subtrees $\left\{s_{1}, s_{2},, s_{n}\right\}$ in a distributed PDCR-tree. Any subtree in $s_{i}, 1 \leq i \leq n$ is called a branch. All nodes of the branch are also nodes of $s_{i}$. The root of the branch is either an internal node of $s_{i}$ or is the same as the root of $s_{i}$. In addition, a branch rooted at a directory node $A$ is called branch $A$.

\section{Lemma 1:}

Assume subtree $s$ with queries traversing inside it, and a branch rooted at node $B$ in sub-tree $s$. In case of updates in the branch and node $B$ (e.g. insertions causing splits), queries need horizontal links to/from $B$ to find new sibling nodes and push them into their stacks for reprocessing.

Proof: Horizontal links are used in query algorithms.

\section{Theorem 1:}

Assumption: Assume $A$ and $C$ as left and right sibling nodes of $B$ in $s$, respectively (Figure A.1). In addition, assume $P$ as the parent node of $A, B, C$. A vertical split as the result of a horizontal split in node $B$, creates a node $D$ which replaces node $B$ and is set to be the 
parent of node $B$ and its new sibling node $B^{\prime}$.

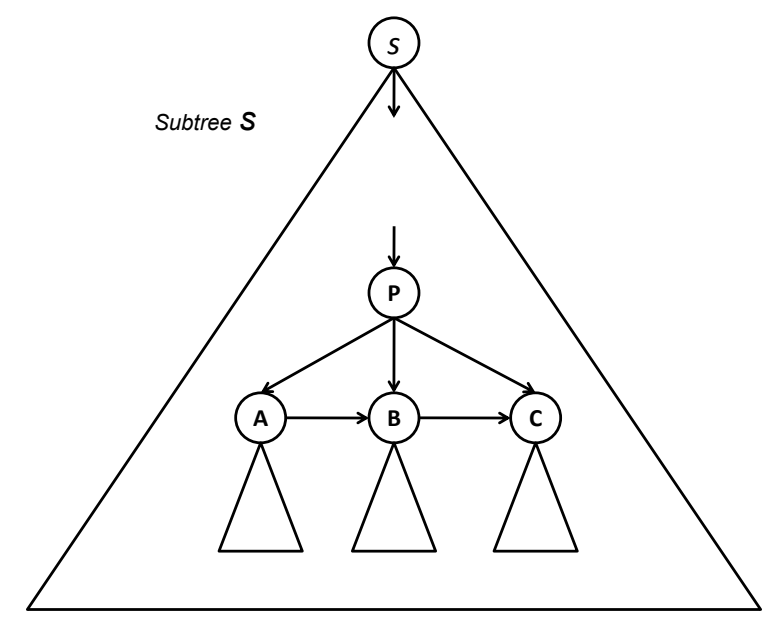

Figure A.1: A subtree $s$ and branch $P$ inside it.

Proposition: Any query $Q$ traversing subtree $s$, does not need the horizontal links $a$, and $c$ to detect the updates in the branch rooted at $D$.

Proof: Assume $B$ as the modified node $B$ after the updates, and $B_{\text {stack }}$ as the old node $B$ pushed into the stack before the updates.

Case 1: Query $Q$ does not have $B$ or one of its ancestors in its stack.

1. The query does not cover the area covered by $B$. Therefore, $Q$ does not care about any updates in $B$.

2. The query has not yet visited node $B$ to push it into its stack. In this case, the query will first visit the newly created node $D$ and pushes it into the stack. This will automatically cover all children of $D$ including $B$ and $B^{\prime}$.

Case 2: Query $Q$ has $B$ or one of its ancestors in its stack.

1. If $Q$ pops node $A$ from the stack, it has not yet popped $B$ from its stack. Once it popped $B_{\text {stack }}, Q$ compares the time stamps of $B$ and $B_{\text {stack }}$ and detects the difference, hence it pushes $B$ and $B^{\prime}$ using the horizontal link $b$ for reprocessing. Node $C$ is still 
in the stack and will be popped later. Therefore, the horizontal links $a$ and $c$ are not used for reprocessing.

2. If $Q$ pops node $B$, it compares the time stamp of $B_{\text {stack }}$ and $B$. $Q$ detects the difference between the time stamps, and pushes $B$ and $B^{\prime}$ using the horizontal links between $B$ and $B^{\prime}$ into the stack for reprocessing. Note that it is not possible to pop node $B^{\prime}$ since it did not exist in the tree when $Q$ visited node $B$ before the updates, and was not pushed into the stack. Node $C$ is still in the stack and will be popped later. Therefore, the horizontal links $a$ and $c$ are not used for reprocessing.

3. If $Q$ pops node $C$, it ignores any update in $B$, since these updates occurred after the processing of the branch $B$ was completed. The horizontal links $a$ and $c$ are not used for reprocessing.

4. If an ancestor of $B$ is in the stack, $B$ will be pushed later into the stack and cases 1-3 will be applied to it.

Hence, in all possible cases, we proved that the updates are considered, and updated nodes are pushed into the stack for reprocessing.

\section{Lemma 2:}

Referring to Theorem 1, there is no need to create new horizontal links from nodes $A$ to $D$, and from nodes $D$ to $C$ to cover updates that occurred in the meantime.

Proof: The proof is straightforward since in all cases mentioned in Theorem 1, queries are able to detect updates with the horizontal link $b$, and using their time stamps. Hence, there is no need to create horizontal links between nodes $A, D$, and $C$.

\section{Definition 2: Independent Branch}

A branch in subtree $s$ is called independent, if its root node is created as the result of a vertical split.

\section{Definition 3: Independent Subtree}

A subtree $s$ from the set $\left\{s_{1}, s_{2},, s_{n}\right\}$ in a worker node, is called independent, if it is also an 
independent branch. As defined before, the root of the subtree has a parent in the hat.

\section{Definition 4: Neighbor Branch}

Two branches are neighbors if:

1. Their roots are two sibling nodes rooted at the same parent node.

2. There is no node in the branches that belongs to both of them. They do not share any node between them.

\section{Corollary 1:}

According to Theorem 1, the horizontal link from other branches (e.g. A) to the old root of an independent branch (i.e. link $a$ ) can be safely removed, since it is not used for reprocessing of the updated nodes. For the same reason, the horizontal link from the new sibling node $(B)$ to other branches (i.e. link $c$ ) can be safely removed (Figure A.2). This leads us to the final structure shown in Figure A.3.

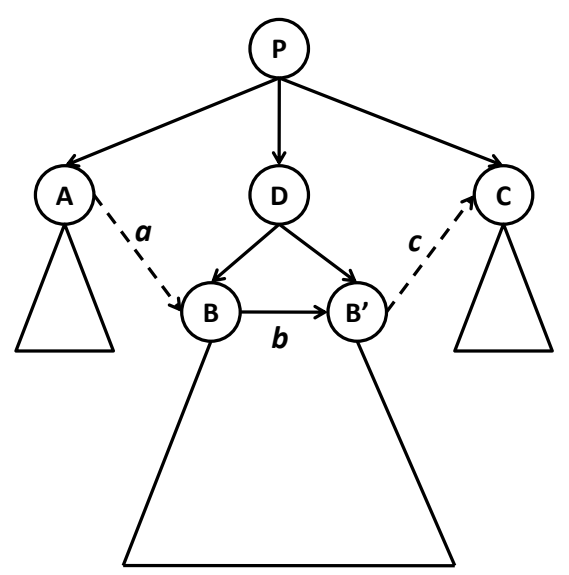

Figure A.2: Branch $P$ before removing horizontal links $a$ and $c$.

\section{Theorem 2:}

Assumption: Assume subtree $s$ and the branch $P$ with nodes in Theorem 1 .

Proposition: If the root of a branch is created from a vertical split (independent branch), there cannot be any horizontal link between descendants of the root and the nodes in neighbor branches. In the case of Theorem 1, there will not be any horizontal link between the 


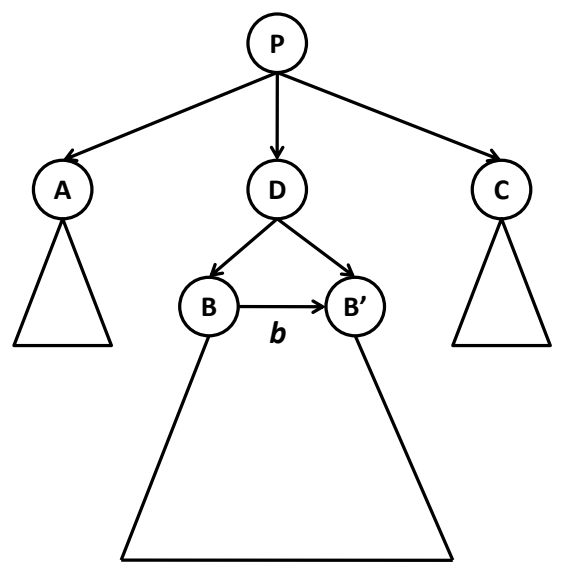

Figure A.3: Branch $P$ after removing horizontal links $a$ and $c$.

nodes in branch $D$ and the nodes in branches $A$ and $C$.

Proof: Since the root of the branch is created from a recent vertical split, it has only two children. Let us call the root as $D$ and its children as $B$ and $B^{\prime}$. Initially the depth of the branch $D$ is 3 , because $B$ and $B^{\prime}$ only have data nodes as children. It was proved in Theorem 1 that the horizontal links from neighbor branches to/from $B$ and $B^{\prime}$ can be safely removed. In addition, it was shown in Lemma 1 that there is no need to create new horizontal links between $D$ and neighbor branches (Figure A.4). Therefore, the proof is correct for the depth of 3.

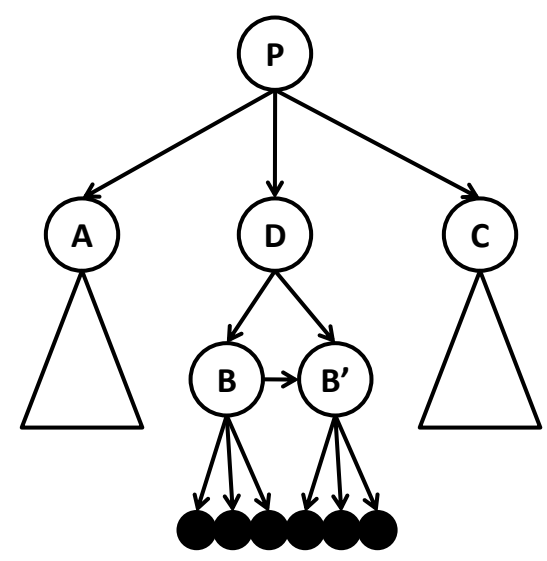

Figure A.4: Branch $P$ before horizontal split in node $B$. 
In order for the branch $D$ to become larger and deeper than depth 3, nodes of the branch must undergo horizontal and vertical splits. Now we consider the creation of new horizontal links in case of both splits to show that no horizontal link is created to/from neighbor branches as the branch becomes larger and deeper.

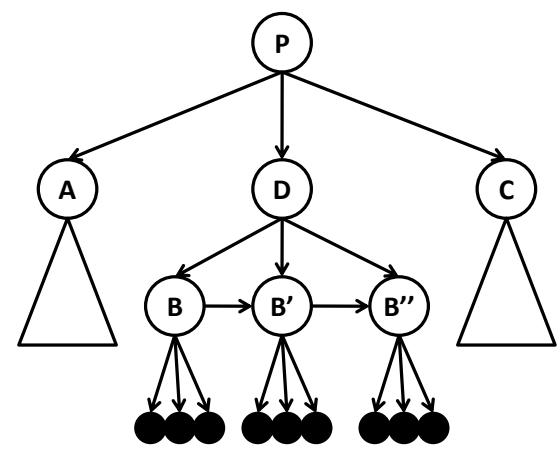

Figure A.5: Branch $P$ after horizontal split in node $B$. Node $B^{\prime}$ is created as the result of the split.

Assume $h$ as the depth of the branch $D$. For each level $i, 1 \leq i \leq d$, in branch $D$ :

1. In case of a horizontal split, the level becomes wider by the horizontal split; however it does not get connected to neighbor branches. Therefore, a horizontal split does not create horizontal links to neighbor branches in any given level $i$. This case is illustrated in Figure A.5.

2. In case of a vertical split, as proved in Theorem 1 and Lemma 2, the new node created from the split, does not create horizontal links to/from any sibling node. Therefore, it does not create any horizontal link to neighbor branches in the level $i$. In addition, children of this node are moved down to the level $i+1$ in the tree and they remove their horizontal links to/from the sibling nodes of their parent in the level $i$. Therefore, no horizontal link will be created to neighbor branches by a vertical split in the levels $i$ and $i+1$. This case is illustrated in Figures A.6 and A.7.

In both cases above, we showed that if branch $D$ becomes wider or deeper by one level, no horizontal link is created to/from sibling nodes of $D$ (i.e. $A$ and $C$ ). In general, as branch $D$ becomes deeper from depth $i$ to $i+1$, no horizontal link is created to/from sibling nodes 


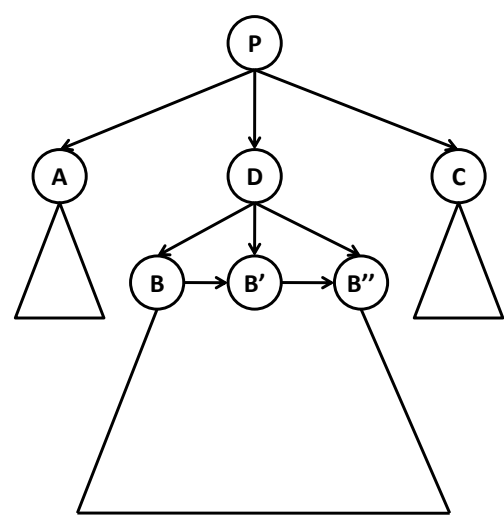

Figure A.6: Branch $P$ and its child branch rooted at node $D$. Note nodes in branch $D$ do not have any link to the neighbor bracnhes $A$ and $C$.

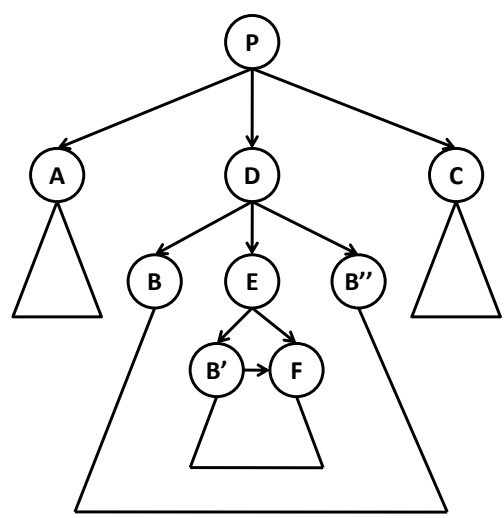

Figure A.7: A vertical split at node $B^{\prime}$ created node $E$. Note branch $E$ is now independent from neighbors.

of $D$ in depth $i$. This can be extended for all levels as follows. For all levels $i$ in branch $D, 1 \leq i \leq d$, no horizontal link is created to/from descendants of neighbor branches $A$ and $C$. New horizontal links are only created between the new descendants inside the branch D.

\section{Observation 1:}

Since there is no horizontal link between an independent branch and its neighbor branches, the independent branch can migrate to other cloud nodes keeping only one vertical connection from a leaf node in the hat to the root of the branch. 


\section{Bibliography}

[1] Amazon elastic compute cloud (ec2) available at http://aws.amazon.com/ec2/.

[2] Bigquery available at http://developers.google.com/bigquery/.

[3] Hadoop available at http://hadoop.apache.org/.

[4] MCSTL: The multi-core standard template library, http://algo2.iti.kit.edu/singler/mcstl/.

[5] Mysql database management system available at http://mysql.com.

[6] NVIDIA CUDA Programming Guide. nVIDIA Corporation, www.nvidia.com.

[7] NVIDIA GPU Technical Specifications. nVIDIA Corporation, www.nvidia.com.

[8] PMSTXXL: Multi-core standard template library for extra large data sets (combination of STXXL and MCSTL).

[9] STXXL: Standard template library for extra large data sets, http://stxxl.sourceforge.net/.

[10] Twitter storm available at http://storm-project.net/.

[11] Zeromq socket library as a concurrency framework available at http://www.zeromq.org/.

[12] The OpenCL Specification 1.0. Khronos OpenCL Working Group, 2009.

[13] A. Abouzeid, K. Bajda-Pawlikowski, D. Abadi, A. Silberschatz, and A. Rasin. Hadoopdb: an architectural hybrid of mapreduce and dbms technologies for analytical workloads. Proc. VLDB Endow., 2(1):922-933, Aug. 2009.

[14] S. Agarwal, R. Agrawal, P. Deshpande, A. Gupta, J. Naughton, R. Ramakrishnan, and S. Sarawagi. On the computation of multidimensional aggregates. Proc. 22nd International VLDB Conference, pages 506-521, 1996.

[15] U. Ahmed, A. Tchounikine, M. Miquel, and S. Servigne. Real-time temporal data warehouse cubing. In Proceedings of the 21 st international conference on Database and expert systems applications: Part II, 2010.

[16] G. M. Amdahl. Validity of the single processor approach to achieving large scale computing capabilities. In Proceedings of the April 18-20, 1967, spring joint computer conference, AFIPS '67 (Spring), pages 483-485, New York, NY, USA, 1967. ACM. 
[17] A. Asiki, K. Doka, D. Tsoumakos, and N. Koziris. Support for concept hierarchies in dhts. In Proceedings of the 2008 Eighth International Conference on Peer-to-Peer Computing, P2P '08, pages 121-124, Washington, DC, USA, 2008. IEEE Computer Society.

[18] A. Asiki, D. Tsoumakos, and N. Koziris. An adaptive online system for efficient processing of hierarchical data. In Proceedings of the 18th ACM international symposium on High performance distributed computing, HPDC '09, pages 71-80, New York, NY, USA, 2009. ACM.

[19] A. Asiki, D. Tsoumakos, and N. Koziris. Distributing and searching concept hierarchies: an adaptive dht-based system. Cluster Computing, 13:257-276, 2010.

[20] X. L. B. Huang, Jinlan Gao. An empirically optimized radix sort for gpu. In IEEE International Symposium on Parallel and Distributed Processing with Applications, 2009.

[21] D. Banks. High-Concurrency Locking in R-Trees. VLDB, pages 1-12, 1995.

[22] A. Beckmann, J. Singler, R. Dementiev, and U. K. (th. Building a parallel pipelined external memory algorithm library. In In 23rd IEEE International Parallel \& Distributed Processing Symposium (IPDPS), 2009.

[23] N. Beckmann, H.-P. Kriegel, R. Schneider, and B. Seeger. The r*-tree: an efficient and robust access method for points and rectangles. In INTERNATIONAL CONFERENCE ON MANAGEMENT OF DATA, pages 322-331. ACM, 1990.

[24] M. Ben-Ari. Principles of Concurrent and Distributed Programming (2nd Edition) (Prentice-Hall International Series in Computer Science). Addison-Wesley Longman Publishing Co., Inc., Boston, MA, USA, 2006.

[25] S. Berchtold, D. A. Keim, and H.-P. Kriegel. The X-tree: An index structure for high-dimensional data. $V L D B$, pages 28-39, Oct. 1996.

[26] K. Beyer and R. Ramakrishnan. Bottom-up computation of sparse and iceberg cubes. Proc. ACM SIGMOD Conference, pages 359-370, 1999.

[27] G. Bilardi and A. Nicolau. Adaptive bitonic sorting. An optimal parallel algorithm for shared-memory machines. SIAM J Comput, 18(2):216-228, 1989.

[28] R. Bruckner, B. List, and J. Schiefer. Striving towards near real-time data integration for data warehouses. DaWaK, LNCS 2454:173-182, 2002.

[29] D. Cederman and P. Tsigas. A practical quicksort algorithm for graphics processors. In Proc. European Symposium on Algorithms (ESA), volume 5193 of LNCS, pages 246-258, 2008. 
[30] D. Cederman and P. Tsigas. GPU-Quicksort. Journal of Experimental Algorithmics, 14(1):1.4, Dec. 2009.

[31] K. Chakrabarti. Efficient Concurrency Control in Multidimensional Access Methods. ACM SIGMOD, pages 25-36, 1999.

[32] F. Chang, J. Dean, S. Ghemawat, W. C. Hsieh, D. A. Wallach, M. Burrows, T. Chandra, A. Fikes, and R. E. Gruber. Bigtable: A distributed storage system for structured data. ACM Trans. Comput. Syst., 26(2):4:1-4:26, June 2008.

[33] Y. Chen, F. Dehne, T. Eavis, and A. Rau-Chaplin. Parallel ROLAP data cube construction on shared-nothing multiprocessors. Distributed and Parallel Databases, 15:219-236, 2004.

[34] F. Dehne, T. Eavis, S. Hambrusch, and A. Rau-Chaplin. Parallelizing the datacube. Proc. Int.Conference on Database Theory, 2001.

[35] F. Dehne, T. Eavis, and A. Rau-Chaplin. A cluster architecture for parallel data warehousing. Proc. Int. Conference on Cluster Computing and the Grid (CCGRID 2001), 2001.

[36] F. Dehne, T. Eavis, and A. Rau-Chaplin. Coarse grained parallel on-line analytical processing (OLAP) for data mining. Proc. Int. Conference on Computational Science (ICCS 2001), pages 589-598, 2001.

[37] F. Dehne, T. Eavis, and A. Rau-Chaplin. Computing partial data cubes for parallel data warehousing applications. Proc. Euro PVM/MPI 2001, 2001.

[38] F. Dehne, T. Eavis, and A. Rau-Chaplin. Parallelizing the datacube. Distributed and Parallel Databases, 11(2):181-201, 2002.

[39] F. Dehne, T. Eavis, and A. Rau-Chaplin. Distributed multi-dimensional ROLAP indexing for the data cube. Proc. IEEE/ACM Int. Symposium on Cluster Computing and the Grid (CCGrid 2003), 2003.

[40] F. Dehne, T. Eavis, and A. Rau-Chaplin. Top-down computation of partial ROLAP data cubes. Proc. Hawaii Int. Conference on System Sciences (HICSS 2004), 2004.

[41] F. Dehne and H. Zaboli. Parallel real-time olap on multi-core processors. In Proceedings of the 2012 12th IEEE/ACM International Symposium on Cluster, Cloud and Grid Computing (ccgrid 2012), pages 588-594, 2012.

[42] R. Dementiev, L. Kettner, and P. Sanders. Stxxl: standard template library for xxl data sets. Software: Practice and Experience, 38 (6), 2008. 
[43] K. Doka, A. Asiki, D. Tsoumakos, and N. Koziris. Online querying of concept hierarchies in $\mathrm{p} 2 \mathrm{p}$ systems. In R. Meersman and Z. Tari, editors, On the Move to Meaningful Internet Systems: OTM 2008, volume 5331 of Lecture Notes in Computer Science, pages 212-230. Springer Berlin / Heidelberg, 2008.

[44] K. Doka, D. Tsoumakos, and N. Koziris. Brown dwarf: A fully-distributed, faulttolerant data warehousing system. Journal of Parallel and Distributed Computing, 71(11):1434 - 1446, 2011.

[45] K. Doka, D. Tsoumakos, and N. Koziris. Online querying of d-dimensional hierarchies. Journal of Parallel and Distributed Computing, 71(3):424 - 437, 2011.

[46] A. S. G. Duane G. Merrill. Revisiting sorting for gpgpu stream architectures. In Proceedings of the 19th international conference on Parallel architectures and compilation techniques, 2010.

[47] M. Ester, J. Kohlhammer, and H.-P. Kriegel. The DC-tree: a fully dynamic index structure for data warehouses. 16th International Conference on Data Engineering (ICDE), pages 379-388, 2000.

[48] A. R.-C. H. Z. R. Z. F. Dehne, Q. Kong. A distributed tree data structure for realtime olap on cloud architectures. In Proceedings of IEEE Int. Conference on Big Data (IEEE BigData 2013), IEEE BigData 2013, pages 499-505. IEEE Computer Society, 2013.

[49] F.Dehne and H.Zaboli. Parallel data cubes on multi-core processors with multiple disks. In in Proc. CASCON 2011, ACM Dig. Library, 2011.

[50] F.Dehne and H.Zaboli. Deterministic sample sort for gpus. Parallel Processing Letters, 22:1250008, 2012.

[51] F.Dehne and H.Zaboli. Parallel construction of data cubes on multi-core multi-disk platforms. Parallel Processing Letters, 23:1350002, 2013.

[52] S. W. B. C. O. M. T. z. Gang Chen, Hoang Tam Vo. A framework for supporting dbms-like indexes in the cloud. In Proceedings of the 6th conference on Symposium on Opearting Systems Design \& Implementation - Volume 6, 2011.

[53] M. Garland. Private communication. nVIDIA Corporation, 2010.

[54] N. Govindaraju, J. Gray, R. Kumar, and D. Manocha. GPUTeraSort: high performance graphics co-processor sorting for large database management. In Proc. International Conference on Management of Data (SIGMOD), pages 325 - 336, 2006.

[55] GPGPU.org. Cudpp library. http://gpgpu.org/developer/cudpp. 
[56] J. Gray, A. Bosworth, A. Layman, and H. Pirahesh. Data cube: A relational aggregation operator generalizing group-by, cross-tab, and sub-totals. Proc. Int. Conference On Data Engineering, pages 152-159, 1996.

[57] J. Gray, S. Chaudhuri, A. Bosworth, A. Layman, D. Reichart, M. Venkatrao, F. Pellow, and H. Pirahesh. Data Cube: A Relational Aggregation Operator Generalizing Group-By, Cross-Tab, and Sub-Totals. Data Min. Know. Disc., 1:29-53, 1997.

[58] A. Greb and G. Zachmann. GPU-ABiSort: Optimal parallel sorting on stream architectures. In Proc. Int'l Parallel and Distributed Processing Symposium (IPDPS), 2006.

[59] Z. Guoliang and C. Hong. Parallel cube computation on modern cpus and gpus. The Journal of Supercomputing, pages 1-24, 2011. 10.1007/s11227-011-0575-7.

[60] A. Guttman. R-trees: a dynamic index structure for spatial searching. ACM SIG$M O D$, pages 47-57, 1984.

[61] J. Han and M. Kamber. Data Mining: Concepts and Techniques. Morgan Kaufmann Publishers, 2000.

[62] S. Hao, Z. Du, B. D.A., and Y. Ye. A partition-merge based cache-conscious parallel sorting algorithm for $\mathrm{cmp}$ with shared cache. Parallel Processing, 2009. ICPP '09. International Conference on, pages 396-403, 2009.

[63] V. Harinarayan, A. Rajaraman, and J. Ullman. Implementing data cubes. Proceedings of the 1996 ACM SIGMOD Conference, pages 205-216, 1996.

[64] J. R. Haritsa, S. Member, and S. Seshadri. Real-Time Index Concurrency Control. IEEE Transactions on Knowledge and Data Engineering, 12(3):429-447, 2000.

[65] H.Peters, O.Schulz-Hildebrandt, and N.Luttenberger. Fast in-place, comparisonbased sorting with cuda: a study with bitonic sort. J.Concurrency and Computation: Practice and Experience, 23:681-693, 2011.

[66] Y. F. X. W. Hua Luan, Mingquan Zhou. Closed cube computation on multi-core cpus. In 9th International Conference on Fuzzy Systems and Knowledge Discovery (FSKD), 2012.

[67] L. Z. Jiang. Olap database computation with a splitcube on a multi-core system, 2009.

[68] D. Jin and T. Tsuji. Parallel data cube construction based on an extendible multidimensional array. In Proceedings of the 2011IEEE 10th International Conference on Trust, Security and Privacy in Computing and Communications, TRUSTCOM '11, pages 1139-1145, Washington, DC, USA, 2011. IEEE Computer Society. 
[69] D. Jin, T. Tsuji, and K. Higuchi. An Incremental Maintenance Scheme of Data Cubes and Its Evaluation. DASFAA, LNCS 4947:36-48, 2008.

[70] Jing Dai. Efficient Concurrent Operations in Spatial Databases. PhD Thesis, Virginia Polytechnic, 2009.

[71] H.-G. Kang and C.-W. Chung. Exploiting versions for on-line data warehouse maintenance in molap servers. In Proceedings of the 28th international conference on Very Large Data Bases, VLDB '02, pages 742-753. VLDB Endowment, 2002.

[72] W. Knight. Two heads are better than one. IEE Review, 51:32-35, 2005.

[73] M. Kornacker and D. Banks. High-concurrency locking in r-trees. 1995.

[74] M. C. Kurt and G. Agrawal. A fault-tolerant environment for large-scale query processing. In High Performance Computing (HiPC), 2012 19th International Conference on, pages 1-10, 2012.

[75] R. K. K.V., S. D., and S. A.K. Improved concurrency control techniques for multidimensional index structures. 1998.

[76] O. P. L. Arge and J. S. Vitter. Implementing i/o-efficient data structures using tpie. In 10th European Symposium on Algorithms (ESA), 2002.

[77] L. Lakshmanan, J. Pei, and J. Han. Quotient cube: How to summarize the semantics of a data cube. Proceedings of the 28th VLDB Conference, 2002.

[78] L. V. S. Lakshmanan, J. Pei, and Y. Zhao. Qc-trees: an efficient summary structure for semantic olap. In proceedings of the 2003 ACM SIGMOD international conference on Management of data, SIGMOD '03, pages 64-75, New York, NY, USA, 2003.

[79] Leda library available at http://www.mpi-sb.mpg.de/leda/.

[80] M. L. Lee, W. Hsu, C. S. Jensen, B. Cui, and K. L. Teo. Supporting Frequent Updates in R-Trees : A Bottom-Up Approach. VLDB, 2003.

[81] N. Leischner, V. Osipov, and P. Sanders. GPU sample sort. In Proc. Int'l Parallel and Distributed Processing Symposium (IPDPS), pages 1-10, 2010.

[82] C multithread library (libmt). http://www.libmt.sourceforge.net.

[83] E. Lindholm, J. Nickolls, S. Oberman, and J. Montrym. NVIDIA Tesla: A unified graphics and computing architecture. IEEE Micro, 28(2):39-55, 2008.

[84] H. Luan, X.-Y. Du, and S. Wang. Cache-conscious data cube computation on a modern processor. Journal of Computer Science and Technology, 24:708-722, 2009. 10.1007/s11390-009-9253-0. 
[85] D. T. Marr, F. Binns, D. L. Hill, G. Hinton, D. A. Koufaty, A. J. Miller, and M. Upton. Hyper-Threading Technology Architecture and Microarchitecture. Intel Technology Journal, 6(1):4-15, Feb. 2002.

[86] S. Melnik, A. Gubarev, J. J. Long, G. Romer, S. Shivakumar, M. Tolton, and T. Vassilakis. Dremel: interactive analysis of web-scale datasets. Proc. VLDB Endow., 3(1-2):330-339, Sept. 2010.

[87] nVIDIA Corporation. Gtx 690 GPU specification. http://www.geforce.com/hardware/desktop-gpus/geforce-gtx-690/specifications.

[88] The OLAP Report. http://www.olapreport.com.

[89] H. Plattner and A. Zeier. In-Memeory Data Management. Springer Verlag, 2011.

[90] K. Ross and D. Srivastava. Fast computation of sparse data cubes. Proceedings of the 23rd VLDB Conference, pages 116-125, 1997.

[91] N. Roussopoulos. Cubetree: Organization of and bulk incremental updates on the data cube. In Proceedings of the 1997 ACM SIGMOD Conference, pages 89-99, 1997.

[92] N. Roussopoulos, Y. Kotidis, and M. Roussopolis. Cubetree: Organization of the bulk incremental updates on the data cube. Proceedings of the 1997 ACM SIGMOD Conference, pages 89-99, 1997.

[93] N. Roussopoulos and D. Leifker. Direct spatial search on pictorial databases using packed r-trees. In Proceedings of the 1985 ACM SIGMOD international conference on Management of data, SIGMOD '85, pages 17-31, New York, NY, USA, 1985. ACM.

[94] R. Santos and J. Bernardino. Real-time data warehouse loading methodology. IDEAS, pages 49-58, 2008.

[95] R. J. Santos and J. Bernardino. Optimizing data warehouse loading procedures for enabling useful-time data warehousing. IDEAS, pages 292-299, 2009.

[96] S. Sarawagi, R. Agrawal, and A.Gupta. On computing the data cube. Technical Report RJ10026, IBM Almaden Research Center, San Jose, California, 1996.

[97] N. Satish. Fast sort on cpus and gpus: a case for bandwidth oblivious simd sort. Proceedings of the 2010 international conference on Management of data, 2010.

[98] N. Satish, M. Harris, and M. Garland. Designing efficient sorting algorithms for manycore GPUs. In Proc. Int'l Parallel and Distributed Processing Symposium (IPDPS), 2009. 
[99] N. Satish, C. Kim, J. Chhugani, A. D. Nguyen, V. W. Lee, D. Kim, and P. Dubey. Fast sort on cpus and gpus: a case for bandwidth oblivious simd sort. In Proc. International Conference on Management of Data (SIGMOD), 2010.

[100] T. Sellis, N. Roussopoulos, and C. Faloutsos. The r+-tree: A dynamic index for multi-dimensional objects. pages 507-518, 1987.

[101] Z. Shao, J. Han, and D. Xin. Mm-cubing: Computing iceberg cubes by factorizing the lattice space. In the Proceedings of the 16th International Conference on Scientific and Statitistical Database Management (SSDBM, pages 213-222, 2004.

[102] H. Shi and J. Schaeffer. Parallel sorting by regular sampling. J. Par. and Dist. Comp., 14:362 - 372, 1992.

[103] Y. X. J. Z. P.-A. H. Shifu Chen, Jing Qin. A fast and flexible sorting algorithm with cuda. Lecture Notes in Computer Science, 5574:281-290, 2009.

[104] E. Sintorn and U. Assarsson. Fast parallel GPU-sorting using a hybrid algorithm. $J$. of Parallel and Distributed Computing, 68(10):1381-1388, 2008.

[105] Y. Sismanis, A. Deligiannakis, Y. Kotidis, and N. Roussopoulos. Hierarchical dwarfs for the rollup cube. In Proceedings of the 6th ACM international workshop on Data warehousing and OLAP, DOLAP '03, pages 17-24, New York, NY, USA, 2003. ACM.

[106] Y. Sismanis, A. Deligiannakis, N. Roussopoulos, and Y. Kotidis. Dwarf: Shrinking the petacube. In Proceedings of the 2002 ACM SIGMOD Conference, pages 464475, 2002.

[107] S. I. Song, Y. H. Kim, and J. S. Yoo. An enhanced concurrency control scheme for multidimensional index structures. IEEE Transactions on Knowledge and Data Engineering archive Volume 16 Issue 1, 2004.

[108] A. Thusoo, J. S. Sarma, N. Jain, Z. Shao, P. Chakka, S. Anthony, H. Liu, P. Wyckoff, and R. Murthy. Hive: a warehousing solution over a map-reduce framework. Proc. VLDB Endow., 2(2):1626-1629, Aug. 2009.

[109] Transaction Processing Performance Council. TPC-DS (Decision Support) Benchmark. http://www.tpc.org/tpcds/tpcds.asp.

[110] J. S. Vitter. Algorithms and Data Structures for External Memory. Foundations and Trends in Theoretical Computer Science, 2008.

[111] J. Wang, S. Wu, H. Gao, J. Li, and B. C. Ooi. Indexing multi-dimensional data in a cloud system. In Proceedings of the 2010 ACM SIGMOD International Conference on Management of data, pages 591-602, 2010. 
[112] W. Wang, J. Feng, H. Lu, and J. Yu. Condensed cube: An effective approach to reducing data cube size. Proceedings of the International Conference on Data Engineering, 2002.

[113] Z. M. Weidong Sun. Count sort for gpu computing. In 15th International Conference on Parallel and Distributed Systems (ICPADS), 2009.

[114] S. Wu, D. Jiang, B. C. Ooi, and K.-L. Wu. Efficient b-tree based indexing for cloud data processing. Proc. VLDB Endow., 3(1-2):1207-1218, Sept. 2010.

[115] S. Wu and K. lung Wu. An indexing framework for efficient retrieval on the cloud. IEEE Data Engineering Bulletin, page 2009.

[116] W. L. N. Y. P. I. Xiaochun Ye, Dongrui Fan. High performance comparison-based sorting algorithm on many-core gpus. In IEEE International Symposium on Parallel and Distributed Processing (IPDPS), 2010.

[117] D. Xin, J. Han, X. Li, and B. W. Wah. Star-cubing: computing iceberg cubes by top-down and bottom-up integration. In Proceedings of the 29th international conference on Very large data bases - Volume 29, VLDB '03, pages 476-487. VLDB Endowment, 2003.

[118] D. Xin, Z. Shao, J. Han, and H. Liu. C-cubing: Efficient computation of closed cubes by aggregation-based checking. In In ICDE06, page 4. IEEE Computer Society, 2006.

[119] X. Zhang, J. Ai, Z. Wang, J. Lu, and X. Meng. An efficient multi-dimensional index for cloud data management. In Proceedings of the first international workshop on Cloud data management, pages 17-24, 2009.

[120] Y. Zhang, S. Wang, and W. Huang. Paracube: A scalable olap model based on distributed aggregate computing with sibling cubes. In Proceedings of the 2010 12th International Asia-Pacific Web Conference, APWEB '10, pages 323-329, Washington, DC, USA, 2010. IEEE Computer Society.

[121] Y. Zhao, P. Deshpande, and J. Naughton. An array-based algorithm for simultaneous multi-dimensional aggregates. Proceedings of the 1997 ACM SIGMOD Conference, pages 159-170, 1997. 